Prepared in cooperation with the U.S. Navy

Distribution of Chlorinated Volatile Organic Compounds and Per- and Polyfluoroalkyl Substances in Monitoring Wells at the Former Naval Air Warfare Center, West Trenton, New Jersey, 2014-17
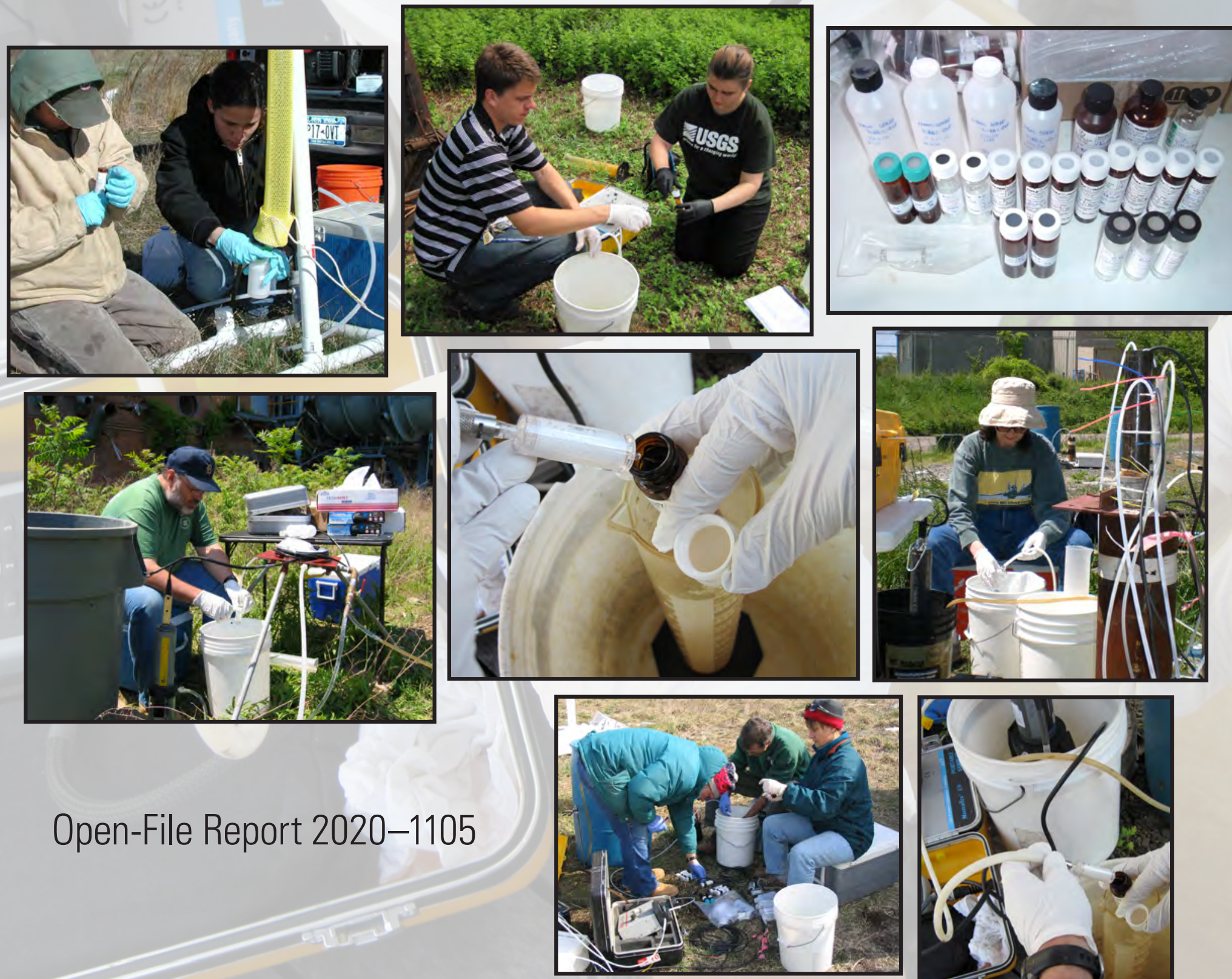

Open-File Report 2020-1105
U.S. Department of the Interior
U.S. Geological Survey

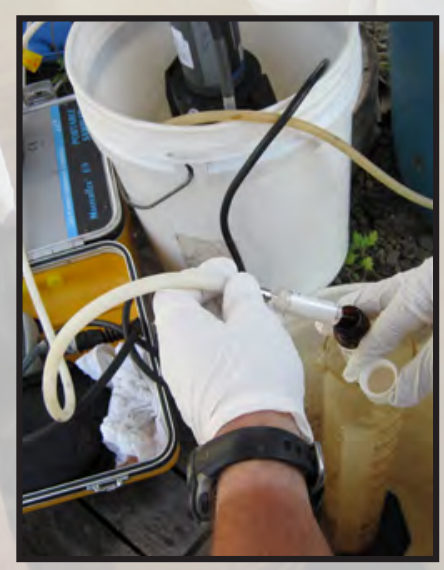




\section{Cover:}

Top left photo: EA Engineering, Science, and Technology, Inc., employees sampling from a USGS regenerated cellulose dialysis membrane passive sampler at the former Naval Air Warfare Center (NAWC), West Trenton, NJ in 2002. Photograph by Thomas E. Imbrigiotta, U.S. Geological Survey.

Top center photo: Christopher L. Leach, USGS intern, and Mary Masse, USGS volunteer, sampling for volatile organic compounds (VOCs) using a peristaltic pump at the former NAWC, West Trenton, NJ in 2014. Photograph by Daniel J. Goode, U.S. Geological Survey.

Top right photo: Bottle set for bioaugmentation sampling at the former NAWC 2010. Photograph by Daniel J. Goode, U.S. Geological Survey.

Center left photo: Thomas E. Imbrigiotta, USGS, sampling VOCs at the former NAWC in 2005. Photograph by Daniel J. Goode, U.S. Geological Survey.

Center center photo: Collection of bacterial DNA sample on a 0.2-micron filter and stable isotopes in the VOC vial at the former NAWC in 2010. Photograph by Daniel J. Goode, U.S. Geological Survey.

Center left photo: Kinga M. Revesz, USGS, collecting samples for carbon isotopes at the former NAWC in 2008. Photograph by Daniel J. Goode, U.S. Geological Survey.

Bottom center photo: Thomas E. Imbrigiotta, USGS, Paul M. Bradley, USGS, and May A. Voytek, USGS, collect bioaugmentation study samples at the former NAWC in 2008. Photograph by Daniel J. Goode, U.S. Geological Survey.

Bottom right photo: Low-flow purge sample collection using a peristaltic pump while monitoring field physical and chemical parameters using a multiparameter sonde in a flow cell at land surface at the former NAWC in 2010. Photograph by Daniel J. Goode, U.S. Geological Survey. 


\section{Distribution of Chlorinated Volatile Organic Compounds and Per- and Polyfluoroalkyl Substances in Monitoring Wells at the Former Naval Air Warfare Center, West Trenton, New Jersey, 2014-17}

By Thomas E. Imbrigiotta and Alex R. Fiore

Prepared in cooperation with the U.S. Navy

Open-File Report 2020-1105 


\section{U.S. Geological Survey, Reston, Virginia: 2021}

For more information on the USGS - the Federal source for science about the Earth, its natural and living resources, natural hazards, and the environment-visit https://www.usgs.gov or call 1-888-ASK-USGS.

For an overview of USGS information products, including maps, imagery, and publications, visit https://store.usgs.gov/.

Any use of trade, firm, or product names is for descriptive purposes only and does not imply endorsement by the U.S. Government.

Although this information product, for the most part, is in the public domain, it also may contain copyrighted materials as noted in the text. Permission to reproduce copyrighted items must be secured from the copyright owner.

Suggested citation:

Imbrigiotta, T.E., and Fiore, A.R., 2021, Distribution of chlorinated volatile organic compounds and per- and polyfluoroalkyl substances in monitoring wells at the former Naval Air Warfare Center, West Trenton, New Jersey, 2014-17: U.S. Geological Survey Open-File Report 2020-1105, 107 p., https://doi.org/10.3133/ofr20201105.

Associated data for this publication:

Fiore, A.R., and Imbrigiotta, T.E., 2021, Concentrations of chlorinated volatile organic compounds and per- and polyfluoroalkyl substances in groundwater and surface water, former Naval Air Warfare Center, West Trenton, New Jersey, U.S. Geological Survey data release, https://doi.org/10.5066/P9RCA05N.

ISSN 2331-1258 (online) 


\section{Acknowledgments}

The authors thank Watermark Environmental for sampling wells and analyzing water-quality samples in 2014, 2015, and 2016. The authors also thank Tetra Tech for sampling wells and analyzing water-quality samples during 2017. Thanks go to Rebecca Talbot, Matt Pronschinske, Savannah Miller, and Jeremy Patterson of the U.S. Geological Survey (USGS) for inputting the water-quality results to the USGS Naval Air Warfare Center database and making geographic information system plots. 



\section{Contents}

Acknowledgments ……...................................................................................................................

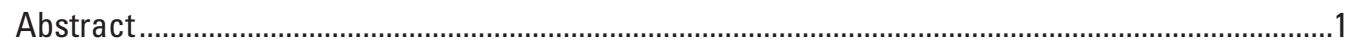

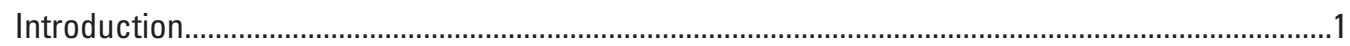

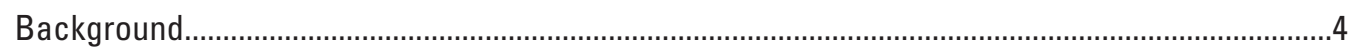

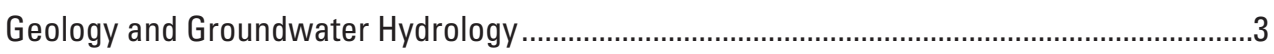

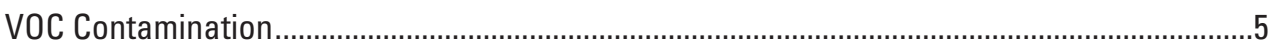

Fate and Transport of VOCs.........................................................................................

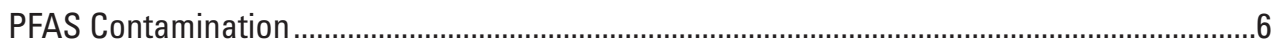

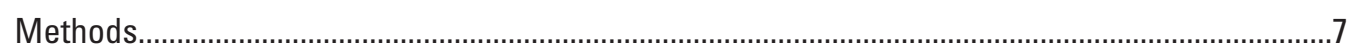

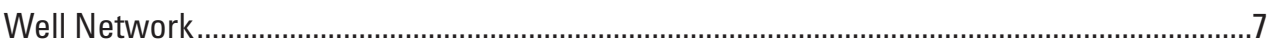

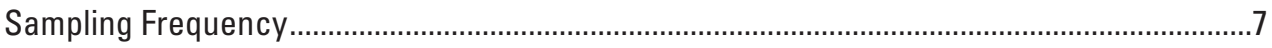

Sample Collection and Analysis .............................................................................................

Field Sampling Procedures for VOCs and 1,4-dioxane ......................................................

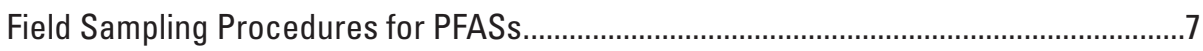

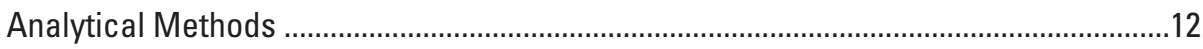

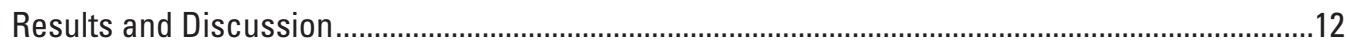

Areal Extent of VOC Contamination at NAWC During 2014-17 ..............................................12

Areal Extent of TCE Contamination During 2014-17..................................................13

Areal Extent of cisDCE Contamination During 2014-17 ...............................................13

Areal Extent of VC Contamination During 2014-17.....................................................18

Vertical Extent of VOC Contamination at NAWC During 2014-17 ..........................................2

Vertical Extent of TCE Contamination During 2014-17................................................27

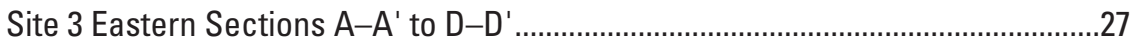

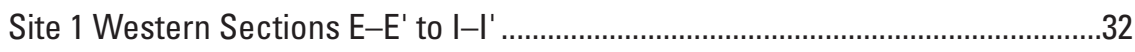

Vertical Extent of cisDCE Contamination During 2014-17 ..............................................32

Site 3 Eastern Sections A-A' to D-D' ...................................................................

Site 1 Western Sections E-E' to I-I' .....................................................................38

Vertical Extent of VC Contamination During 2014-17........................................................38

Site 3 Eastern Sections $A-A^{\prime}$ to $D-D^{\prime}$......................................................................4

Site 1 Western Sections $E-E^{\prime}$ to $I-I^{\prime}$.....................................................................48

Variation in VOC Contamination over Time ........................................................................4

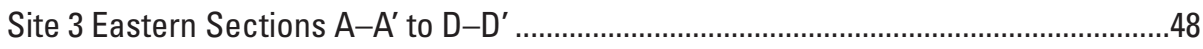

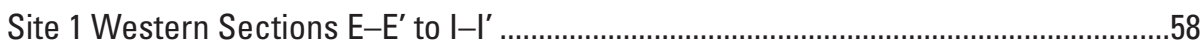

PFAS Contamination at the Former NAWC Site During 2015-17............................................59

Areal Extent of PFAS Contamination in Groundwater During 2015-17 ….......................59

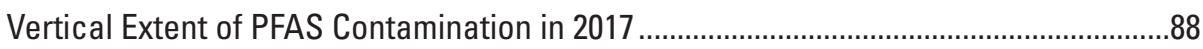

Sampling Method Comparison for PFASs.....................................................................8

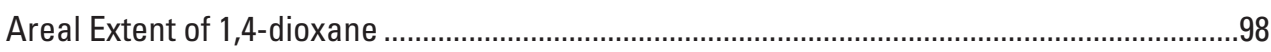

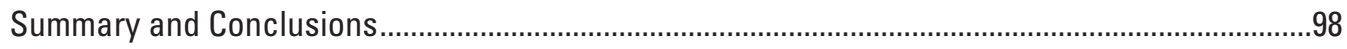

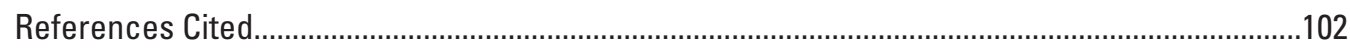

Appendix 1. Descriptions of boreholes, well locations, and well construction at the former Naval Air Warfare Center, West Trenton, New Jersey............................................106 
Appendix 2. Volatile organic compounds, per- and polyfluoroalkyl substances, and 1,4-dioxane concentrations measured in samples from wells at the former Naval Air Warfare Center site, West Trenton, New Jersey, 1990-2017

\section{Figures}

1. Map showing well locations and source areas of volatile organic compounds and per- and polyfluoroalkyl substances at the former Naval Air Warfare Center, West Trenton, New Jersey.

2. Map showing the generalized groundwater flow directions, surface water features, locations of pump-and-treat wells, volatile organic compound and per- and polyfluoroalkyl substance source areas, and former research study sites, at the former Naval Air Warfare Center, West Trenton, New Jersey. .4

3. Conceptual dip-aligned geologic cross section of the former Naval Air Warfare Center, West Trenton, New Jersey.

4. Map showing areal extent of trichloroethene contamination in wells at the former Naval Air Warfare Center, West Trenton, New Jersey, 2014

5. Map showing areal extent of trichloroethene contamination in wells at the former Naval Air Warfare Center, West Trenton, New Jersey, 2015

6. Map showing areal extent of trichloroethene contamination in wells at the former Naval Air Warfare Center, West Trenton, New Jersey, 2016

7. Map showing areal extent of trichloroethene contamination in wells at the former Naval Air Warfare Center, West Trenton, New Jersey, 2017

8. Map showing areal extent of cis-1,2-dichloroethene contamination in wells at the former Naval Air Warfare Center, West Trenton, New Jersey, 2014.

9. Map showing areal extent of cis-1,2-dichloroethene contamination in wells at the former Naval Air Warfare Center, West Trenton, New Jersey, 2015.

10. Map showing areal extent of cis-1,2-dichloroethene contamination in wells at the former Naval Air Warfare Center, West Trenton, New Jersey, 2016.

11. Map showing areal extent of cis-1,2-dichloroethene contamination in wells at the former Naval Air Warfare Center, West Trenton, New Jersey, 2017.

12. Map showing areal extent of vinyl chloride contamination in wells at the former Naval Air Warfare Center, West Trenton, New Jersey, 2014

13. Map showing areal extent of vinyl chloride contamination in wells at the former Naval Air Warfare Center, West Trenton, New Jersey, 2015

14. Map showing areal extent of vinyl chloride contamination in wells at the former Naval Air Warfare Center, West Trenton, New Jersey, 2016.

15. Map showing areal extent of vinyl chloride contamination in wells at the former Naval Air Warfare Center, West Trenton, New Jersey, 2017

16. Cross section showing vertical extent of trichloroethene concentrations in wells along section $A-A^{\prime}$ in $A, 2014$ and $B, 2015-17$, former Naval Air Warfare Center, West Trenton, New Jersey.

17. Cross section showing vertical extent of trichloroethene concentrations in wells along section B-B' in $A, 2014$ and B, 2015-17, former Naval Air Warfare Center, West Trenton, New Jersey.

18. Cross section showing vertical extent of trichloroethene concentrations in wells along section $C-C^{\prime}$ in $A, 2014$ and $B, 2015-17$, former Naval Air Warfare Center, West Trenton, New Jersey. 
19. Cross section showing vertical extent of trichloroethene concentrations in wells along section D-D' in $A, 2014$ and $B, 2015-17$, former Naval Air Warfare Center, West Trenton, New Jersey.

20. Cross section showing vertical extent of trichloroethene concentrations in wells along section $\mathrm{E}-\mathrm{E}^{\prime}$ in $A, 2014$ and $B, 2015-17$, former Naval Air Warfare Center, West Trenton, New Jersey....

21. Cross section showing vertical extent of trichloroethene concentrations in wells along section $\mathrm{F}^{-} \mathrm{F}^{\prime}$ in $A, 2014$ and $B, 2015-17$, former Naval Air Warfare Center, West Trenton, New Jersey

22. Cross section showing vertical extent of trichloroethene concentrations in wells along section $\mathrm{G}-\mathrm{G}^{\prime}$ in $A, 2014$ and $B, 2015-17$, former Naval Air Warfare Center, West Trenton, New Jersey.

23. Cross section showing vertical extent of trichloroethene concentrations in wells along section $\mathrm{H}^{-\mathrm{H}^{\prime}}$ in $A, 2014$ and $B, 2015-17$, former Naval Air Warfare Center, West Trenton, New Jersey.

24. Cross section showing vertical extent of trichloroethene concentrations in wells along section I-I' in $A, 2014$ and B, 2015-17, former Naval Air Warfare Center, West Trenton, New Jersey.

25. Cross section showing vertical extent of cis-1,2-dichloroethene concentrations in wells along section A- $A^{\prime}$ in A, 2014 and B, 2015-17, former Naval Air Warfare Center, West Trenton, New Jersey

26. Cross section showing vertical extent of cis-1,2-dichloroethene concentrations in wells along section B-B' in A, 2014 and B, 2015-17, former Naval Air Warfare Center, West Trenton, New Jersey

27. Cross section showing vertical extent of cis-1,2-dichloroethene concentrations in wells along section $C-C^{\prime}$ in $A, 2014$ and $B, 2015-17$, former Naval Air Warfare Center, West Trenton, New Jersey

28. Cross section showing vertical extent of cis-1,2-dichloroethene concentrations in wells along section D-D' in A, 2014 and B, 2015-17, former Naval Air Warfare Center, West Trenton, New Jersey

29. Cross section showing vertical extent of cis-1,2-dichloroethene concentrations in wells along section $\mathrm{E}-\mathrm{E}$ ' in $A, 2014$ and $B, 2015-17$, former Naval Air Warfare Center, West Trenton, New Jersey

30. Cross section showing vertical extent of cis-1,2-dichloroethene concentrations in wells along section $\mathrm{F}-\mathrm{F}^{\prime}$ in $A, 2014$ and $B, 2015-17$, former Naval Air Warfare Center, West Trenton, New Jersey

31. Cross section showing vertical extent of cis-1,2-dichloroethene concentrations in wells along section $\mathrm{G}_{-} \mathrm{G}^{\prime}$ in $A, 2014$ and $B, 2015-17$, former Naval Air Warfare Center, West Trenton, New Jersey....

32. Cross section showing vertical extent of cis-1,2-dichloroethene concentrations in wells along section $\mathrm{H}-\mathrm{H}^{\prime}$ in $A, 2014$ and $B, 2015-17$, former Naval Air Warfare Center, West Trenton, New Jersey....

33. Cross section showing vertical extent of cis-1,2-dichloroethene concentrations in wells along section I-I' in A, 2014 and B, 2015-17, former Naval Air Warfare Center, West Trenton, New Jersey.

34. Cross section showing vertical extent of vinyl chloride concentrations in wells along section $\mathrm{A}-\mathrm{A}^{\prime}$ in $A, 2014$ and $B, 2015-17$, former Naval Air Warfare Center, West Trenton, New Jersey. 
35. Cross section showing vertical extent of vinyl chloride concentrations in wells along section B-B' in A, 2014 and $B, 2015-17$, former Naval Air Warfare Center, West Trenton, New Jersey.

36. Cross section showing vertical extent of vinyl chloride concentrations in wells along section $C-C^{\prime}$ in $A, 2014$ and $B, 2015-17$, former Naval Air Warfare Center, West Trenton, New Jersey.

37. Cross section showing vertical extent of vinyl chloride concentrations in wells along section D-D' in A, 2014 and B, 2015-17, former Naval Air Warfare Center, West Trenton, New Jersey.

38. Cross section showing vertical extent of vinyl chloride concentrations in wells along section $\mathrm{E}-\mathrm{E}^{\prime}$ in $A, 2014$ and $B, 2015-17$, former Naval Air Warfare Center, West Trenton, New Jersey.

39. Cross section showing vertical extent of vinyl chloride concentrations in wells along section $\mathrm{F}-\mathrm{F}^{\prime}$ in $A, 2014$ and $B, 2015-17$, former Naval Air Warfare Center, West Trenton, New Jersey.

40. Cross section showing vertical extent of vinyl chloride concentrations in wells along section $\mathrm{G}_{-} \mathrm{G}^{\prime}$ in $A, 2014$ and $B, 2015-17$, former Naval Air Warfare Center, West Trenton, New Jersey.

41. Cross section showing vertical extent of vinyl chloride concentrations in wells along section $\mathrm{H}-\mathrm{H}^{\prime}$ in $A, 2014$ and $B, 2015-17$, former Naval Air Warfare Center, West Trenton, New Jersey.

42. Cross section showing vertical extent of vinyl chloride concentrations in wells along section I-I' in A, 2014 and B, 2015-17, former Naval Air Warfare Center, West Trenton, New Jersey.

43. Graphs showing volatile organic compound concentrations over time in wells along section $A-A^{\prime}$, former Naval Air Warfare Center, West Trenton, New Jersey, 1992-2017.

44. Graphs showing volatile organic compound concentrations over time in wells along section B-B', former Naval Air Warfare Center, West Trenton, New Jersey, 1990-2017

45. Graphs showing volatile organic compound concentrations over time in wells along section C-C', former Naval Air Warfare Center, West Trenton, New Jersey 1992-2017

46. Graphs showing volatile organic compound concentrations over time in wells along section D-D', former Naval Air Warfare Center, West Trenton, New Jersey, 1992-2017

47. Graphs showing volatile organic compound concentrations over time in wells along section E-E', former Naval Air Warfare Center, West Trenton, New Jersey, 1992-2017

48. Graphs showing volatile organic compound concentrations over time in wells along section $\mathrm{F}-\mathrm{F}^{\prime}$, former Naval Air Warfare Center, West Trenton, New Jersey, 1992-2017

49. Graphs showing volatile organic compound concentrations over time in wells along section $\mathrm{G}-\mathrm{G}$ ', former Naval Air Warfare Center, West Trenton, New Jersey, 1992-2017

50. Graphs showing volatile organic compound concentrations over time in wells along section $\mathrm{H}-\mathrm{H}^{\prime}$, former Naval Air Warfare Center, West Trenton, New Jersey, 1992-2017. 
51. Graphs showing volatile organic compound concentrations over time in wells along section I-I', former Naval Air Warfare Center, West Trenton, New Jersey, 1993-2017.

52. Map showing areal extent of perfluorooctane sulfonate concentrations in wells at the former Naval Air Warfare Center, West Trenton, New Jersey, 2016

53. Map showing areal extent of perfluorooctanoic acid concentrations in wells at the former Naval Air Warfare Center, West Trenton, New Jersey, 2016.

54. Map showing areal extent of perfluorononanoic acid concentrations in wells at the former Naval Air Warfare Center, West Trenton, New Jersey, 2016.

55. Map showing areal extent of perfluorooctane sulfonate concentrations in wells at the former Naval Air Warfare Center, West Trenton, New Jersey, 2017

56. Map showing areal extent of perfluorooctanoic acid concentrations in wells at the former Naval Air Warfare Center, West Trenton, New Jersey, 2017

57. Map showing areal extent of perfluorononanoic acid concentrations in wells at the former Naval Air Warfare Center, West Trenton, New Jersey, 2017

58. Cross section showing vertical extent of maximum perfluorooctane sulfonate concentrations in wells along sections $A-A^{\prime}$ and $B-B^{\prime}$, former Naval Air Warfare Center, West Trenton, New Jersey, 2015-17.

59. Cross section showing vertical extent of perfluorooctane sulfonate concentrations in wells along sections $C-C^{\prime}$ and $D-D^{\prime}$, former Naval Air Warfare Center, New Jersey, 2015-17

60. Cross section showing vertical extent of perfluorooctanoic acid concentrations in wells along sections $A-A^{\prime}$ and $B-B^{\prime}$, former Naval Air Warfare Center, West Trenton, New Jersey, 2015-17.

61. Cross section showing vertical extent of perfluorooctanoic acid concentrations in wells along sections $C-C^{\prime}$ and $D^{-} D^{\prime}$, former Naval Air Warfare Center, West Trenton, New Jersey, 2015-17.

62. Cross section showing vertical extent of perfluorononanoic acid concentrations in wells along sections $A-A^{\prime}$ and $B-B^{\prime}$, former Naval Air Warfare Center, West Trenton, New Jersey, 2015-17

63. Cross section showing vertical extent of perfluorononanoic acid concentrations in wells along sections $C-C^{\prime}$ and $D-D^{\prime}$, former Naval Air Warfare Center, West Trenton, New Jersey, 2015-17

64. Graph showing concentrations of perfluorooctane sulfonate (PFOS) in samples collected using regenerated cellulose dialysis membrane samplers in relation to concentrations of PFOS from low-flow purging, with a 1:1 linear relation line

65. Graph showing concentrations of perfluorooctanoic acid (PFOA) in samples collected using regenerated cellulose dialysis membrane samplers in relation to concentrations of PFOA from low-flow purging, with a 1:1 linear relation line.

66. Graph showing concentrations of perfluorononanoic acid (PFNA) in samples collected using regenerated cellulose dialysis membrane samplers in relation to concentrations of PFNA from low-flow purging, with a 1:1 linear relation line 100

67. Map showing areal extent of 1,4-dioxane concentrations in wells at the former Naval Air Warfare Center, West Trenton, New Jersey, 2017 


\section{Tables}

1. Sampling frequency for volatile organic compounds and per- and polyfluoroalkyl substances in selected wells at the former Naval Air Warfare Center, West Trenton, New Jersey, 2014-17.

\section{Conversion Factors}

U.S. customary units to International System of Units

\begin{tabular}{|c|c|c|}
\hline Multiply & By & To obtain \\
\hline \multicolumn{3}{|c|}{ Length } \\
\hline inch (in.) & 2.54 & centimeter $(\mathrm{cm})$ \\
\hline foot $(\mathrm{ft})$ & 0.3048 & meter $(\mathrm{m})$ \\
\hline mile (mi) & 1.609 & kilometer $(\mathrm{km})$ \\
\hline \multicolumn{3}{|c|}{ Area } \\
\hline acre & 4,047 & square meter $\left(\mathrm{m}^{2}\right)$ \\
\hline acre & 0.004047 & square kilometer $\left(\mathrm{km}^{2}\right)$ \\
\hline square foot $\left(\mathrm{ft}^{2}\right)$ & 0.09290 & square meter $\left(\mathrm{m}^{2}\right)$ \\
\hline square mile $\left(\mathrm{mi}^{2}\right)$ & 2.590 & square kilometer $\left(\mathrm{km}^{2}\right)$ \\
\hline \multicolumn{3}{|c|}{ Volume } \\
\hline quart (qt) & 0.9464 & liter (L) \\
\hline gallon (gal) & 3.785 & liter $(\mathrm{L})$ \\
\hline gallon (gal) & 0.003785 & cubic meter $\left(\mathrm{m}^{3}\right)$ \\
\hline \multicolumn{3}{|c|}{ Flow rate } \\
\hline gallon per minute (gal/min) & 0.06309 & liter per second $(\mathrm{L} / \mathrm{s})$ \\
\hline \multicolumn{3}{|c|}{ Mass } \\
\hline ounce, avoirdupois (oz) & 28.35 & $\operatorname{gram}(\mathrm{g})$ \\
\hline
\end{tabular}

International System of Units to U.S. customary units

\begin{tabular}{lcl}
\hline \multicolumn{1}{c}{ Multiply } & By & \multicolumn{1}{c}{ To obtain } \\
\hline centimeter $(\mathrm{cm})$ & Length & \\
meter $(\mathrm{m})$ & 0.3937 & inch (in.) \\
kilometer $(\mathrm{km})$ & 3.281 & foot (ft) \\
\hline \multicolumn{2}{c}{ Area } & mile (mi) \\
\hline square meter $\left(\mathrm{m}^{2}\right)$ & 0.6214 & acre \\
square kilometer $\left(\mathrm{km}^{2}\right)$ & 0.0002471 & acre \\
square meter $\left(\mathrm{m}^{2}\right)$ & 247.1 & square foot $\left(\mathrm{ft}^{2}\right)$ \\
square kilometer $\left(\mathrm{km}^{2}\right)$ & 10.76 & square mile $\left(\mathrm{mi}^{2}\right)$ \\
\hline
\end{tabular}




\begin{tabular}{|c|c|c|}
\hline Multiply & By & To obtain \\
\hline \multicolumn{3}{|c|}{ Volume } \\
\hline liter $(\mathrm{L})$ & 33.81402 & ounce, fluid (fl. oz) \\
\hline liter (L) & 1.057 & quart (qt) \\
\hline liter (L) & 0.2642 & gallon (gal) \\
\hline cubic meter $\left(\mathrm{m}^{3}\right)$ & 264.2 & gallon (gal) \\
\hline liter $(\mathrm{L})$ & 61.02 & cubic inch $\left(\right.$ in $\left.^{3}\right)$ \\
\hline cubic meter $\left(\mathrm{m}^{3}\right)$ & 35.31 & cubic foot $\left(\mathrm{ft}^{3}\right)$ \\
\hline \multicolumn{3}{|c|}{ Flow rate } \\
\hline liter per second $(\mathrm{L} / \mathrm{s})$ & 15.85 & gallon per minute (gal/min) \\
\hline \multicolumn{3}{|c|}{ Mass } \\
\hline gram $(\mathrm{g})$ & 0.03527 & ounce, avoirdupois (oz) \\
\hline kilogram (kg) & 2.205 & pound avoirdupois (lb.) \\
\hline
\end{tabular}

Temperature in degrees Celsius $\left({ }^{\circ} \mathrm{C}\right)$ may be converted to degrees Fahrenheit $\left({ }^{\circ} \mathrm{F}\right)$ as follows:

$$
{ }^{\circ} \mathrm{F}=\left(1.8 \times{ }^{\circ} \mathrm{C}\right)+32 \text {. }
$$

Temperature in degrees Fahrenheit $\left({ }^{\circ} \mathrm{F}\right)$ may be converted to degrees Celsius $\left({ }^{\circ} \mathrm{C}\right)$ as follows:

$$
{ }^{\circ} \mathrm{C}=\left({ }^{\circ} \mathrm{F}-32\right) / 1.8 \text {. }
$$

\section{Datum}

Vertical coordinate information is referenced to the North American Vertical Datum of 1988 (NAVD 88).

Horizontal coordinate information is referenced to the North American Datum of 1983 (NAD 83).

Altitude, as used in this report, refers to distance above the vertical datum.

\section{Supplemental Information}

Specific conductance is given in microsiemens per centimeter at 25 degrees Celsius $\left(\mu \mathrm{S} / \mathrm{cm}\right.$ at $\left.25^{\circ} \mathrm{C}\right)$.

Concentrations of chemical constituents in water are given in either milligrams per liter (mg/L), micrograms per liter ( $\mu \mathrm{g} / \mathrm{L})$, or nanograms per liter $(\mathrm{ng} / \mathrm{L})$. 


\section{Abbreviations}

$\begin{array}{ll}\text { AFFF } & \text { aqueous film-forming foam } \\ \text { cisDCE } & \text { cis-1,2-dichloroethene } \\ \text { DNAPL } & \text { dense non-aqueous phase liquid } \\ \text { EPA } & \text { U.S. Environmental Protection Agency } \\ \text { ESO } & \text { emulsified soybean oil } \\ \text { HAL } & \text { health advisory limit } \\ \text { ITRC } & \text { Interstate Technology and Regulatory Council } \\ \text { MCL } & \text { maximum contaminant level } \\ \text { NJDEP } & \text { New Jersey Department of Environmental Protection } \\ \text { NIOSH } & \text { National Institute for Occupational Safety and Health } \\ \text { NAWC } & \text { Naval Air Warfare Center } \\ \text { PFAS } & \text { per- and polyfluoroalkyl substance } \\ \text { PFNA } & \text { perfluorononanoic acid } \\ \text { PFOS } & \text { perfluorooctane sulfonate } \\ \text { PFOA } & \text { perfluorooctanoic acid } \\ \text { RCDM } & \text { Regenerated cellulose dialysis membrane } \\ \text { TCE } & \text { trichloroethene } \\ \text { USGS } & \text { U.S. Geological Survey } \\ \text { VC } & \text { vinyl chloride } \\ \text { VOC } & \text { volatile organic compound }\end{array}$




\title{
Distribution of Chlorinated Volatile Organic Compounds and Per- and Polyfluoroalkyl Substances in Monitoring Wells at the Former Naval Air Warfare Center, West Trenton, New Jersey, 2014-17
}

\author{
By Thomas E. Imbrigiotta and Alex R. Fiore
}

\section{Abstract}

A study was conducted by the U.S. Geological Survey in cooperation with the U.S. Navy (the Navy) to determine the status of volatile organic compounds (VOCs) and per- and polyfluoroalkyl substances (PFASs) in groundwater at the former Naval Air Warfare Center (NAWC) in West Trenton, New Jersey. Wells contaminated with VOCs were sampled in 2014 , 2015, 2016, and 2017 as part of the Navy's long-term monitoring program. The results for trichloroethene (TCE), cis1,2-dichloroethene (cisDCE), and vinyl chloride (VC) were plotted in map view to determine whether the areal extent of the contamination had changed over the 4-year period. TCE, cisDCE, and VC concentrations were plotted along nine lines of section across the former NAWC site to determine whether the vertical distribution of VOCs had changed during 2014-17. TCE, cisDCE, and VC concentrations over time were plotted on graphs for each well to determine long-term trends and changes in VOC concentrations. Data from 1990 to 2017 were used, if available, to make these graphs.

Results show that the areas of VOC concentrations greater than or equal to 1 microgram per liter decreased slightly on the northwestern side and the northeastern side of the NAWC site from 2014 to 2017 under the influence of a pump-and-treat system, natural attenuation processes, and engineered bioaugmentation experiments ongoing at the site. The pump-and-treat system continued to hydraulically contain the VOC contamination and kept it from moving offsite to the south and west of NAWC. One well northeast of the NAWC site, 50BR, was found to have detectable TCE and cisDCE concentrations. These detections indicated that VOC contamination had migrated offsite and that the pump-and-treat system was not containing the VOC contamination on the eastern side of the facility. Detectable VOC concentrations were present in wells as deep as 200 and 221 feet on the eastern and western sides of the NAWC site. TCE concentrations in most wells were found to be stable or to have slowly decreased since the facility closed in 1999. Only 7 wells, including 3 pump-andtreat extraction wells, showed substantial increases in TCE concentration from 2014 to 2017. Continuing sources of TCE to the system are desorption of TCE from organic materials in the aquifer, back diffusion of TCE from the contaminated bedrock matrix, and dissolution of remaining dense nonaqueous phase TCE in the aquifer.

Wells at the former NAWC site were sampled for PFASs in 2015, 2016, and 2017. Perfluorooctane sulfonate (PFOS), perfluorooctanoic acid (PFOA), and perfluorononanoic acid (PFNA) results were plotted in map and cross-section views to determine the areal and vertical extent of the PFAS contamination at the site. PFOS, PFOA, and PFNA concentrations greater than their established maximum contaminant levels were detected in 25,24 , and 21 of the 26 wells sampled, respectively, on the eastern side of NAWC in 2017. Vertically, the highest PFAS concentrations were present in shallow wells along the fence near the firehouse and along the railroad tracks where the aqueous film-forming foam discharge reportedly occurred back in 1990. PFAS concentrations were detected in one well (54BR) as deep as 200 feet on the eastern side of the NAWC site. PFASs were present in wells east of the railroad tracks, indicating that PFAS-contaminated groundwater had moved offsite. In a limited test of five wells, samples collected with regenerated cellulose dialysis membrane (RCDM) passive samplers contained PFAS concentrations equal to those in samples from low-flow purging.

\section{Introduction}

The former Naval Air Warfare Center (NAWC) in West Trenton, New Jersey, was a jet engine test facility for naval aircraft from the mid-1950s to the late 1990s (fig. 1). Activities at the 65 -acre site involved testing the performance of jet engines under different temperatures and pressures. A heating/ cooling system for adjusting the temperature of the air piped into the engine test cells contained 25,000 gallons of trichloroethene (TCE) as the refrigerant (International Technology Corporation, 1994). Over the course of 40 years of testing, 


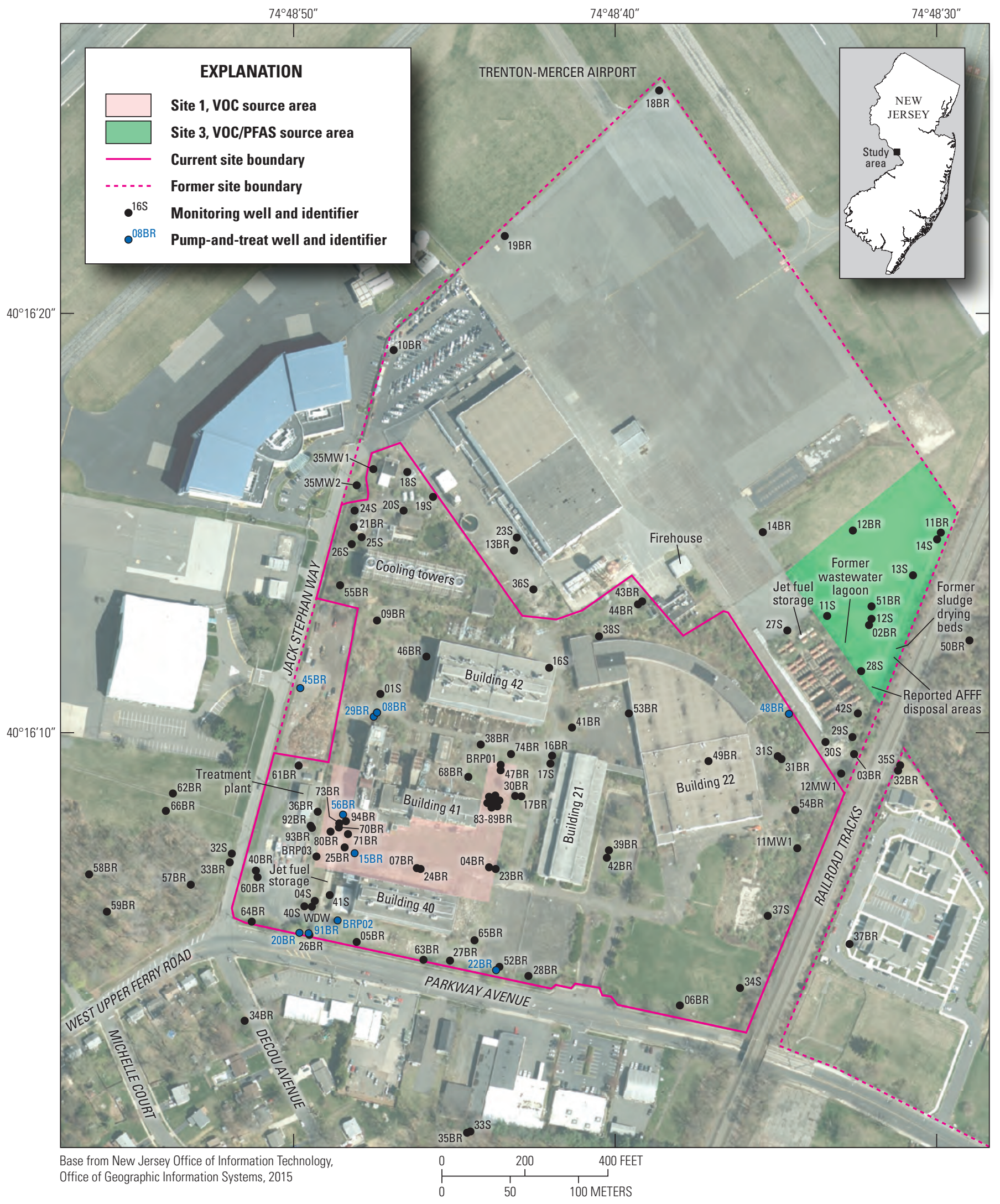

Figure 1. Well locations and source areas of volatile organic compounds and per- and polyfluoroalkyl substances at the former Naval Air Warfare Center, West Trenton, New Jersey. (VOC, volatile organic compound; PFAS, per- and polyfluoroalkyl substance) 
substantial volumes of TCE were spilled or leaked from the system onto the ground, contaminating the fractured rock aquifer beneath the NAWC site.

To prepare for the possibility of having to extinguish aircraft engine fires during testing, personnel at the base fire house were trained in the use of aqueous film-forming foam (AFFF). AFFFs produced in the 1960s to early 1990s contained high concentrations of per- and polyfluoroalkyl substances (PFASs). PFAS contamination was identified in well 48BR in 2015 and in the storm sewers draining the eastern side of the NAWC site in 2016 (P.J. Lacombe, T.E. Imbrigiotta, and Alex Fiore, U.S. Geological Survey, written communication, 2016).

The volatile organic compound (VOC) groundwater contamination at this site has been monitored extensively over the past 25 years by the U.S. Navy (the Navy), its contractors, and the U.S. Geological Survey (USGS). The Navy and the New Jersey Department of Environmental Protection (NJDEP) use these monitoring data to determine whether the contamination is being hydraulically contained and how fast the concentrations are decreasing over time. The PFAS contamination has been monitored in the groundwater only since 2015. The Navy and NJDEP will evaluate the PFAS data to determine the next steps in addressing this contamination.

The USGS, in cooperation with the Navy, has evaluated the results of monitoring efforts at the NAWC site by interpreting the observed changes in chemical contamination areally, vertically, and temporally on a periodic basis. The primary objectives of this report were to (1) determine whether there had been significant changes in the areal extent of the VOC contamination at the NAWC site from 2014 to 2017, (2) determine whether there had been significant changes in the vertical extent of VOC contamination along nine lines of section at the NAWC site from 2014 to 2017, (3) determine whether there had been significant changes in VOC concentrations over time in the wells at NAWC, (4) determine the areal extent of PFAS contamination in the groundwater at the NAWC site, (5) determine the vertical extent of PFAS contamination at the NAWC site, (6) compare the PFAS concentrations in samples collected using two different sampling methods, and (7) determine the areal extent of 1,4-dioxane contamination at the NAWC site. To accomplish these objectives, TCE, cis-1,2-dichloroethene (cisDCE), and vinyl chloride (VC) concentrations in groundwater samples from wells on or near the former NAWC site collected in 2015, 2016, and 2017 were compared to concentrations measured in 2014, which was the last major sampling of wells at NAWC. This report presents areal distributions of VOCs on maps, vertical distributions on cross sections, and temporal variations on graphs showing concentrations over time. For the PFASs, the areal and vertical distribution of perfluorooctane sulfonate (PFOS), perfluorooctanoic acid (PFOA), and perfluorononanoic acid (PFNA) from 2015 to 2017 are presented on maps and cross sections. The areal extent of 1,4-dioxane concentrations for 2017 is presented on a map of the NAWC site.

\section{Background}

\section{Geology and Groundwater Hydrology}

A consolidated fractured sedimentary bedrock aquifer is present below the NAWC site and is composed primarily of mudstones and siltstones of the Lockatong Formation and sandstones of the Stockton Formation (Lacombe, 2000; Lacombe, 2002) (fig. 2). The upper 7-10 feet (ft) of surficial sediments at NAWC are composed primarily of soil and fill material brought in to level the site. Because of bedrock weathering, a layer of unconsolidated saprolite is present down to depths of 25-40 feet below land surface (Lacombe, 2000; Lacombe, 2002). Below this weathered layer, rock cores, drillers logs, and geophysical logs were collected from boreholes drilled on site. Based on the cores and logs, both formations are composed of a series of sloping beds that dip to the northwest and strike northeast-southwest (fig. 3). Logs also indicate that the Lockatong Formation underlies most of the contaminated area at NAWC.

The Stockton and Lockatong Formations are separated by a fault that strikes about $\mathrm{N} 65^{\circ} \mathrm{E}$ and dips $70^{\circ} \mathrm{SW}$ or more (Lacombe and Burton, 2010) (fig. 2). A fault zone abuts the fault and consists of a heterogeneous suite of discontinuous beds from the Lockatong and Stockton Formations that dip in many directions and whose attitudes range from flat lying to overturned (fig. 3). Most beds in the fault zone are heavily brecciated and weathered to clay, creating a low permeability barrier that limits groundwater flow between the Lockatong and Stockton Formations.

Most groundwater flow at NAWC occurs through fractures parallel to the bedding plane along the strike and through dip-aligned fractures between the sloping beds (Lacombe, 2000). In the Lockatong Formation, the most transmissive water-bearing units are six highly fractured fissile black mudstone units identified by Lacombe and Burton (2010) as BlkFis-159, BlkFis-172, BlkFis-190, BlkFis-233, BlkFis-246, and BlkFis 262, which underly most of NAWC including the contaminated areas. The Stockton Formation at NAWC is less well defined compared to the Lockatong Formation. Lacombe (2000) utilized lower-resolution gamma log correlations to divide the Stockton into 5 units (S11, S12, S13, S14, and S15). All these layers are shown on figure 3 and in all subsequent cross sections.

The shallow natural groundwater flow gradients unstressed by the pump-and-treat well pumping are toward the Gold Run Spring west of NAWC and toward the ancestral west branch of Gold Run, which is currently (2020) culverted beneath Parkway Avenue south of NAWC (fig. 2) and discharges into the Delaware River (Lacombe, 2000). Most of the bedrock below about $250 \mathrm{ft}$ is unfractured and yields little water (Lacombe and Burton, 2010). Pumping from the extraction wells that are part of the pump-and-treat system dominates the stressed groundwater gradients and 


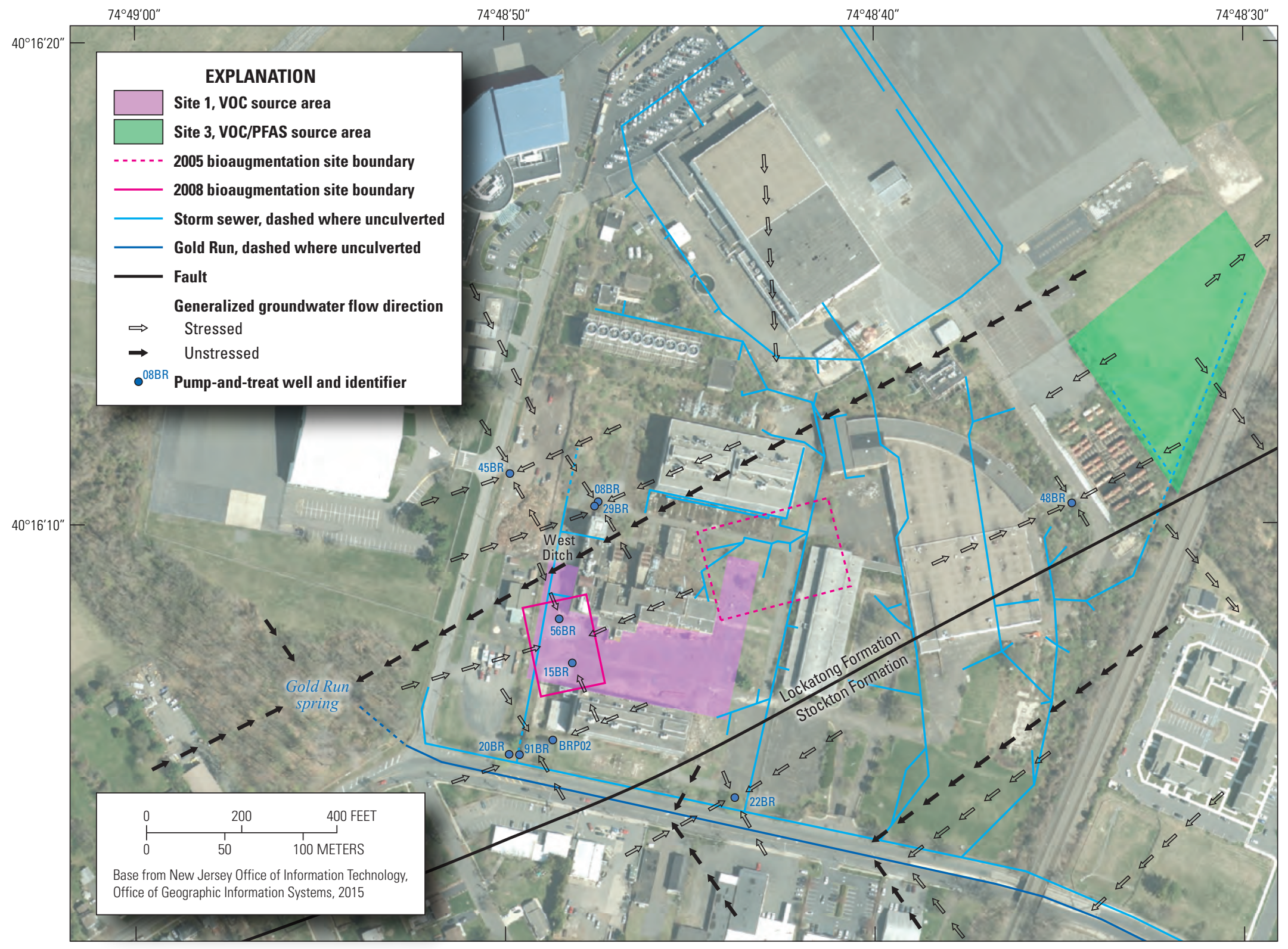

Figure 2. Generalized groundwater flow directions, surface water features, locations of pump-and-treat wells, volatile organic compound and per- and polyfluoroalkyl substance source areas, and former research study sites, at the former Naval Air Warfare Center, West Trenton, New Jersey. (VOC, volatile organic compound; PFAS, perand polyfluoroalkyl substance) 

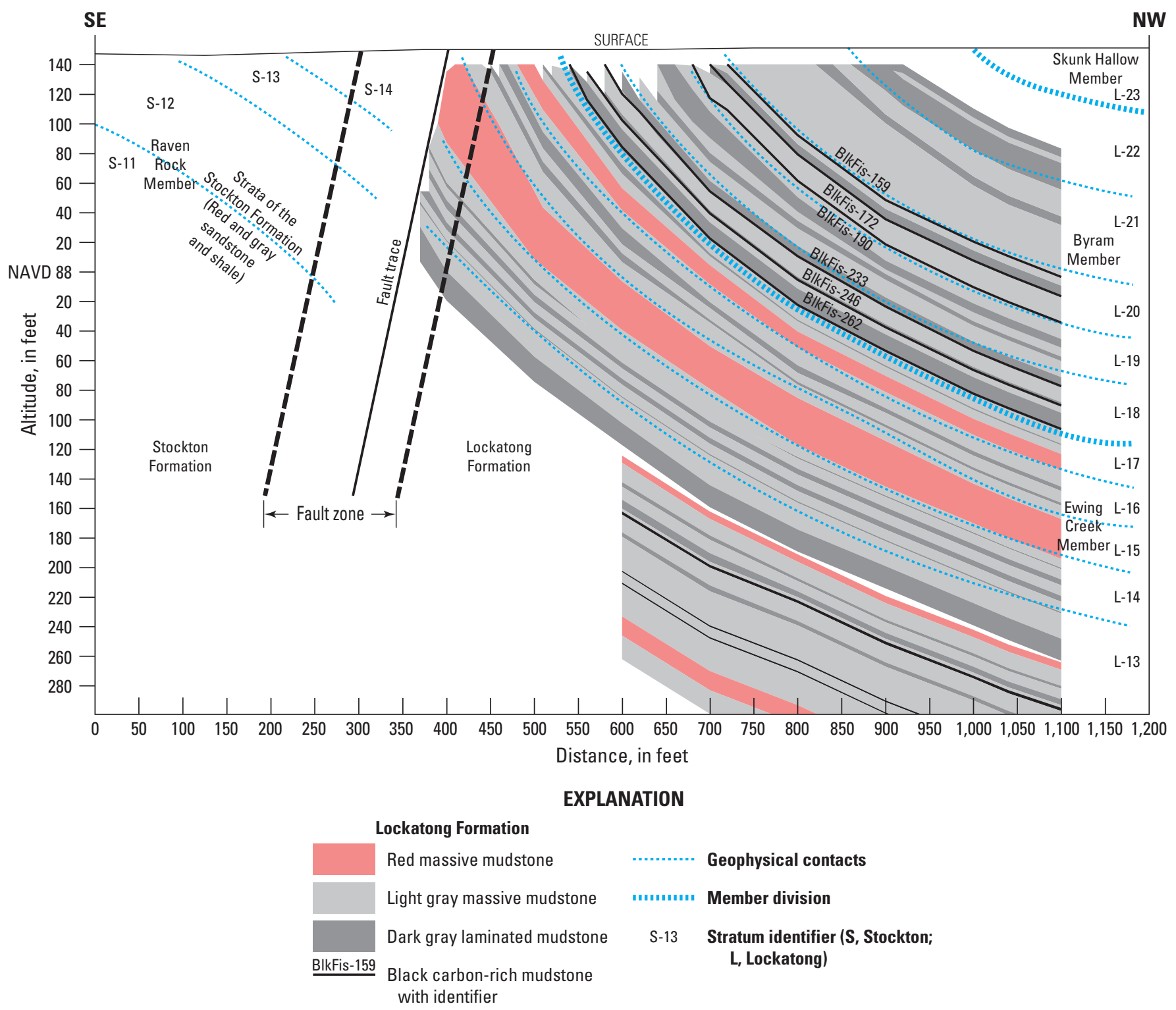

Figure 3. Conceptual dip-aligned geologic cross section of the former Naval Air Warfare Center, West Trenton, New Jersey. Modified from Lacombe and Burton (2010).

local groundwater flow at NAWC (fig. 2). The pump-and-treat system is designed to hydraulically contain the contaminated groundwater on site and prevent its migration to offsite areas.

\section{VOC Contamination}

TCE was first detected in groundwater at NAWC in the late 1980s (International Technology Corporation, 1994). A network of monitoring wells was installed during the next 20 years to further define the extent of VOC groundwater contamination (Appendix 1; fig. 1). Subsequent investigations determined that the bulk of the TCE contamination in the groundwater was in two main areas, (1) the Site 1 area west of the main entrance and (2) the Site 3 area east of the main entrance (fig. 1). The Site 1 area on the western side of NAWC includes the main TCE source area between Building 40 that contained the heating/cooling system and Building 41 that contained the jet engine test cells (fig.1). The Site 3 area on the eastern side of NAWC includes the secondary TCE source areas of a former wastewater lagoon and a sludge drying and disposal area near the railroad tracks that make up the current eastern boundary of the site (fig. 1). The Site 1 area was found to be more highly contaminated (maximum TCE concentrations 750,000 micrograms per liter $(\mu \mathrm{g} / \mathrm{L})$ than the Site 3 area (maximum TCE concentrations 1,300 $\mu \mathrm{g} / \mathrm{L}$ )) (Appendix 2). Biodegradation breakdown products of TCE, specifically 
cisDCE and $\mathrm{VC}$, were also detected at concentrations that far exceeded their NJDEP drinking water maximum contaminant limits (MCLs). NJDEP MCLs for TCE, cisDCE, and VC are 1 $\mu \mathrm{g} / \mathrm{L}, 70 \mu \mathrm{g} / \mathrm{L}$, and $1 \mu \mathrm{g} / \mathrm{L}$, respectively (NJDEP, 2018c).

The Navy and its contractors established a long-term groundwater quality monitoring program in the late 1990s (EA Engineering, Science, and Technology, Inc., 2000; ECOR Solutions, Inc., 2005). Groundwater samples have been collected from wells at the NAWC site every year since then. The results from samples collected from years 2014 through 2017 are discussed in this report.

\section{Fate and Transport of VOCs}

The VOC groundwater contamination at NAWC evolved over the life of the facility in a manner similar to other sites overlying fractured rock aquifers. Typically, TCE was used at a site in its dense non-aqueous phase liquid (DNAPL) form. Because DNAPL TCE is denser than water, when it is spilled onto the ground, it seeps into the subsurface and sinks in the aquifer under the influence of gravity and groundwater flow gradients (Cohen and others, 1993). DNAPL TCE physically travels downward along sloping bedding partings and vertical fractures until it becomes trapped in dead-end fractures or is blocked by a lower permeability layer (Cohen and others, 1993). Once in the aquifer, the DNAPL TCE dissolves into the groundwater flowing through the fractures because it is somewhat soluble $(1,100$ milligrams per liter $(\mathrm{mg} / \mathrm{L})$ ) (National Institute for Occupational Safety and Health [NIOSH], 2007). There were many reports of DNAPL TCE spills and leaks over the history of the facility (EA Engineering, Science, and Technology, Inc., 2000), so the above scenario likely occurred at NAWC, with the TCE used as the refrigerant in the cooling system being the original source.

Once dissolved-phase TCE is in a fractured rock aquifer, it can then be transported and transformed by many other natural processes and human activities (Mackay and others, 1985; Vogel and others, 1987; Wiedemeier and others, 1998). TCE may be advectively transported downgradient to discharge to streams, storm sewers, or extraction wells for treatment systems. TCE may diffuse into the primary porosity of the rock matrix or sorb onto the organic materials present in the geologic formation. Because TCE has a high vapor pressure (NIOSH, 2007), it may volatilize from the groundwater into the soil gas at the interface with the unsaturated zone. In addition, TCE may be biodegraded by natural bacteria in the aquifer or by bacteria that have been injected into the aquifer to form breakdown products, such as cisDCE, VC, and ethene (Wilson and Wilson, 1985; Barrio-Lage and others, 1986; Wilson and others, 1995; Lorah and Olsen, 1999). Researchers working at NAWC have observed evidence that all these processes have occurred at the site (Lacombe, 2011; Bradley and others, 2009; Révész and others, 2014; Goode and others, 2014; Tiedeman and others, 2018; Shapiro and others, 2018).
Because the inputs of TCE from the jet engine test facility were stopped when the NAWC site closed in 1999, the only continuing sources of TCE to the fractured rock aquifer are desorption of TCE from organic materials in the aquifer, back diffusion of TCE from the primary porosity of the rock matrix, and the dissolution of any DNAPL TCE remaining in deadend pore spaces. Because TCE was the original VOC spilled at the site, the presence of cisDCE and VC indicates that anaerobic biodegradation has occurred and is continuing to occur in the aquifer at NAWC.

Several research projects have investigated the potential remediation of the TCE contamination in the bedrock aquifers underlying NAWC over the past 20 years. Two biodegradation studies have been conducted in the Site 1 area of NAWC (fig. 2) where TCE-degrading bacteria and emulsified soybean oil (ESO) have been injected into a few wells (Geosyntec Consultants, 2010; Shapiro and others, 2018). These studies monitored concentrations of TCE and its breakdown products in the injection wells and other nearby wells to determine whether biodegradation was increased by these injections over time.

\section{PFAS Contamination}

The AFFF formulations produced from the 1960s to the 1980s and presumably used in the early 1990s on the eastern side of the former NAWC site contained substantial concentrations of PFOS and PFOA, with smaller concentrations of PFNA (Interstate Technology and Regulatory Council [ITRC], 2018c). PFOS, PFOA, and PFNA are somewhat soluble in groundwater, have very low vapor pressures that prevent these compounds from readily volatilizing to the unsaturated zone, and have high soil organic carbon-water partition coefficients that cause these compounds to be adsorbed to organic materials in the geologic formation (ITRC, 2018a). PFOS, PFOA, and PFNA are not known to undergo biodegradation by microbes in the natural environment (ITRC, 2018b). These properties make these compounds environmentally stable, mobile, and persistent.

Once an aquifer has been contaminated with PFASs, it is very difficult to clean it up to NJDEP or U.S. Environmental Protection Agency (EPA) groundwater quality standards. NJDEP has adopted MCLs for PFNA, PFOS, and PFOA of 13 nanograms per liter $(\mathrm{ng} / \mathrm{L}), 13 \mathrm{ng} / \mathrm{L}$, and $14 \mathrm{ng} / \mathrm{L}$, respectively (NJDEP, 2018c; 2018a; 2018b). The EPA currently does not have MCLs for any PFASs but does have health advisory limits (HALs) for PFOS and PFOA of $70 \mathrm{ng} / \mathrm{L}$ individually and as a combined sum (EPA, 2016).

The source of the PFAS contamination in the Site 3 area of the NAWC site, according to the former fire chief at NAWC from 1985 to 1995, was the test use of one 5-gallon drum of AFFF around 1990. The AFFF was expanded with the addition of the recommended volume of water and then disposed of by spraying it along the fence line east of the firehouse, north of the tank farm, and along the western side of the railroad tracks 
north of the tank farm (George Drew, former NAWC fire chief, oral communication, 2016) (fig. 1). AFFF was reportedly never used in live fire training exercises or to put out any actual fires in the jet engine test cells at the NAWC site.

\section{Methods}

Groundwater quality data were collected from the existing NAWC well network. Field sampling protocols used at this site included purging techniques and passive diffusion sampling methods. Standard laboratory analytical methods were used to analyze the samples.

\section{Well Network}

The entire NAWC well network consists of 137 wells or well zones that are on and around the former NAWC site. The locations and identifiers of these wells are shown in figure 1. The depths and construction details of all network wells at or near the NAWC site are given in Appendix 1. Most of these wells are monitoring wells; however, 10 of the wells are extraction wells for the pump-and-treat system operating at the site. Wells 36BR, 71BR, 73BR, and 80BR contain multiple depth intervals isolated by pneumatic packers. Only the shallow intervals in these four wells are sampled and discussed in this report.

\section{Sampling Frequency}

In 2009, at the request of the Navy, the USGS completed a reassessment of the all wells in the long-term monitoring network at NAWC. Wells were evaluated for importance, based on the concentrations of contaminants present in the well, well location at the site, and well use. As a result of this reassessment and its approval by the NJDEP, the frequency of sampling was adjusted in many wells from annual to biennial or quadrennial, and the constituents monitored were changed. Beginning in 2010, comprehensive sampling of wells has occurred every 4 years; it is based on the Sampling and Analysis Plan developed by the Navy, its contractors, and the USGS and approved by the NJDEP (Tetra Tech, 2010; Watermark Environmental, 2014, 2015a, 2016a; Tetra Tech, 2017). During the comprehensive sampling events (2010 and 2014), most wells in the network were sampled for field physical and chemical characteristics, inorganic constituents, and organic compounds (primarily VOCs). During the sampling events that occurred between these comprehensive sampling events, usually only field physical and chemical characteristics and VOC concentrations were measured, and fewer wells were included. Therefore, the subset of wells sampled differs from year to year.

During 2014, 2015, 2016, and 2017, 94 wells or well zones were sampled for some combination of VOCs, PFASs, and 1,4-dioxane. These wells, their sampling frequency, and the organic compounds sampled during each sampling event are listed in table 1. Inorganic constituents were sampled in 2014 but were not sampled during 2015-17, so no comparisons of inorganic data are made or are discussed in this report.

\section{Sample Collection and Analysis}

\section{Field Sampling Procedures for VOCs and 1,4-dioxane}

Field sampling procedures varied by well according to the protocols laid out in the Sampling and Analysis Plan established each year by the consulting firm in charge of well sampling. Some wells were purged using a "hybrid" purging technique that involves using a variable-speed stainless-steel submersible pump to purge 1.5 casing volumes of water from the well from just above the open interval, then lowering the purge rate to less than 1 liter per minute $(\mathrm{L} / \mathrm{min})$ and monitoring field physical and chemical characteristics (temperature, $\mathrm{pH}$, specific conductance, dissolved oxygen, and turbidity) to stability using a multi-parameter sonde in a flow cell prior to sample collection. Some wells were purged using a low-flow purging technique that involves lowering the pump into the open interval of the well, purging at a flow rate of less than 1 $\mathrm{L} / \mathrm{min}$ from the start, and monitoring field physical and chemical characteristics to stability using a multi-parameter sonde in a flow cell prior to sample collection. In all sampling with a submersible pump, dedicated Teflon-lined polyethylene discharge tubing was used while purging and sampling the wells. Other wells were sampled with polyethylene diffusion bag samplers or regenerated cellulose dialysis membrane (RCDM) diffusion samplers. These passive samplers were constructed and deployed using the procedures detailed in Imbrigiotta and others (2008) and Imbrigiotta and Harte (2020). Passive samplers were equilibrated in each well for approximately 2 weeks prior to retrieval and sample collection. Extraction wells were purged using the installed submersible pumps and were sampled from a tap at the well head after field physical and chemical characteristics were monitored to stability using a multi-parameter sonde in a flow cell.

\section{Field Sampling Procedures for PFASs}

To collect samples for PFASs, wells were purged from the screen or open interval using a low-flow purging technique, which involved pumping with a peristaltic pump and using clean high-density polyethylene discharge tubing at a flow rate of less than $1 \mathrm{~L} / \mathrm{min}$. Field physical and chemical characteristics were monitored to stability using a multiparameter sonde in a flow cell. Once field physical and chemical characteristic stability was reached, PFAS samples were collected in high density polyethylene bottles. PFAS sampling was done at a different time from the VOC sampling to avoid any cross contamination. 
Table 1. Sampling frequency for volatile organic compounds and per- and polyfluoroalkyl substances in selected wells at the former Naval Air Warfare Center, West Trenton, New Jersey, 2014-17.

[bls, below land surface; VOCs, volatile organic compounds; PFASs, per- and polyfluoroalkyl substances; BR, bedrock; MW, monitoring well; S, shallow; X, indicates well was sampled]

\begin{tabular}{|c|c|c|c|c|c|c|c|c|c|c|c|c|c|c|c|c|}
\hline $\begin{array}{c}\text { Local } \\
\text { Identifier }\end{array}$ & $\begin{array}{c}\text { Depth to } \\
\text { top of open } \\
\text { interval, } \\
\text { in feet bls }\end{array}$ & $\begin{array}{l}\text { Depth to } \\
\text { bottom } \\
\text { of open } \\
\text { interval, in } \\
\text { feet bls }\end{array}$ & $\begin{array}{c}\text { Sampling } \\
\text { frequency, } \\
\text { VOCs }\end{array}$ & $\begin{array}{l}\text { VOCs } \\
\text { sam- } \\
\text { pled } \\
\text { spring } \\
2014\end{array}$ & $\begin{array}{l}\text { VOCs } \\
\text { sam- } \\
\text { pled } \\
\text { fall } \\
2014\end{array}$ & $\begin{array}{l}\text { VOCs } \\
\text { sam- } \\
\text { pled } \\
\text { spring } \\
2015\end{array}$ & $\begin{array}{c}\text { VOCs } \\
\text { sam- } \\
\text { pled } \\
\text { fall } \\
2015\end{array}$ & $\begin{array}{l}\text { VOCs } \\
\text { sam- } \\
\text { pled } \\
\text { spring } \\
2016\end{array}$ & $\begin{array}{l}\text { VOCs } \\
\text { sam- } \\
\text { pled } \\
\text { fall } \\
2016\end{array}$ & $\begin{array}{l}\text { VOCs } \\
\text { sam- } \\
\text { pled } \\
\text { spring } \\
2017\end{array}$ & $\begin{array}{l}\text { VOCs } \\
\text { sam- } \\
\text { pled } \\
\text { fall } \\
2017\end{array}$ & $\begin{array}{c}\text { PFASs } \\
\text { sam- } \\
\text { pled } \\
\text { spring } \\
2015\end{array}$ & $\begin{array}{c}\text { PFASs } \\
\text { sam- } \\
\text { pled } \\
\text { fall } \\
2016\end{array}$ & $\begin{array}{l}\text { PFASs } \\
\text { sam- } \\
\text { pled } \\
\text { spring } \\
2017\end{array}$ & $\begin{array}{c}\text { PFASs } \\
\text { sam- } \\
\text { pled } \\
\text { fall } \\
2017\end{array}$ & $\begin{array}{l}\text { 1,4-dioxane } \\
\text { sampled } \\
\text { spring } 2017\end{array}$ \\
\hline 02BR & 40 & 60 & Biennial & $\mathrm{X}$ & & & & $\mathrm{X}$ & & & & & & $\mathrm{X}$ & & $\mathrm{X}$ \\
\hline 03BR & 35 & 45 & Quadrennial & $\mathrm{X}$ & & & & & & & & & & & & \\
\hline 04BR & 24 & 39 & Annual & $\mathrm{X}$ & & $\mathrm{X}$ & & $\mathrm{X}$ & & $\mathrm{X}$ & & & & & & $\mathrm{X}$ \\
\hline 05BR & 69 & 84 & Biennial & $\mathrm{X}$ & & & & $\mathrm{X}$ & & & & & & & & \\
\hline 06BR & 52 & 77 & Biennial & $\mathrm{X}$ & & & & $\mathrm{X}$ & & & & & & $\mathrm{X}$ & & $\mathrm{X}$ \\
\hline 07BR & 38 & 53 & Annual & $\mathrm{X}$ & & $\mathrm{X}$ & & $\mathrm{X}$ & & $\mathrm{X}$ & & & & & & \\
\hline 08BR & 32 & 57 & Semiannual & $\mathrm{X}$ & $\mathrm{X}$ & $\mathrm{X}$ & $\mathrm{X}$ & $\mathrm{X}$ & $\mathrm{X}$ & $\mathrm{X}$ & $\mathrm{X}$ & & $\mathrm{X}$ & & & $\mathrm{X}$ \\
\hline 09BR & 19 & 44 & Biennial & $\mathrm{X}$ & & & & $\mathrm{X}$ & & & & & & & & \\
\hline $10 \mathrm{BR}$ & 63 & 88 & Unscheduled & & & & & & & & & & $\mathrm{X}$ & & & \\
\hline $11 \mathrm{BR}$ & 55 & 75 & Biennial & $\mathrm{X}$ & & & & $\mathrm{X}$ & & & & & $\mathrm{X}$ & $\mathrm{X}$ & & \\
\hline 11MW1 & 8 & 22 & Biennial & $\mathrm{X}$ & & & & $\mathrm{X}$ & & & & & & $\mathrm{X}$ & & \\
\hline $11 \mathrm{~S}$ & 8 & 23 & Quadrennial & $\mathrm{X}$ & & & & & & & & & & $\mathrm{X}$ & & \\
\hline $12 \mathrm{BR}$ & 56.5 & 71.5 & Biennial & $\mathrm{X}$ & & & & $\mathrm{X}$ & & & & & & $\mathrm{X}$ & & \\
\hline $12 \mathrm{~S}$ & 10.5 & 20.5 & Biennial & $\mathrm{X}$ & & & & $\mathrm{X}$ & & & & & & $\mathrm{X}$ & & \\
\hline $13 \mathrm{~S}$ & 10 & 20 & Quadrennial & $\mathrm{X}$ & & & & & & & & & $\mathrm{X}$ & $\mathrm{X}$ & & \\
\hline $14 \mathrm{BR}$ & 42 & 67 & Quadrennial & $\mathrm{X}$ & & & & & & & & & $\mathrm{X}$ & $\mathrm{X}$ & & \\
\hline $14 \mathrm{~S}$ & 14.5 & 24.5 & Quadrennial & $\mathrm{X}$ & & & & & & & & & $\mathrm{X}$ & $\mathrm{X}$ & & \\
\hline $15 \mathrm{BR}$ & 26 & 41 & Semiannual & $\mathrm{X}$ & $\mathrm{X}$ & $\mathrm{X}$ & $\mathrm{X}$ & $\mathrm{X}$ & $X$ & $\mathrm{X}$ & $X$ & & $X$ & & & $\mathrm{X}$ \\
\hline $16 \mathrm{BR}$ & 40 & 65 & Biennial & $\mathrm{X}$ & & & & $\mathrm{X}$ & & & & & & & & \\
\hline $16 \mathrm{~S}$ & 2 & 12 & Biennial & $\mathrm{X}$ & & & & $\mathrm{X}$ & & & & & $\mathrm{X}$ & & & \\
\hline $17 \mathrm{BR}$ & 19 & 44 & Annual & $\mathrm{X}$ & & $\mathrm{X}$ & & $\mathrm{X}$ & & $\mathrm{X}$ & & & & & & \\
\hline $18 \mathrm{BR}$ & 27 & 52 & Unscheduled & & & & & & & & & & $\mathrm{X}$ & & & \\
\hline 19BR & 43 & 58 & Biennial & $\mathrm{X}$ & & & & $\mathrm{X}$ & & & & & $\mathrm{X}$ & & & $\mathrm{X}$ \\
\hline 20BR & 28 & 43 & Semiannual & $\mathrm{X}$ & $\mathrm{X}$ & $X$ & $\mathrm{X}$ & $\mathrm{X}$ & $\mathrm{X}$ & $\mathrm{X}$ & $\mathrm{X}$ & & $\mathrm{X}$ & & & $\mathrm{X}$ \\
\hline $21 \mathrm{BR}$ & 50 & 65 & Biennial & $\mathrm{X}$ & & & & $\mathrm{X}$ & & & & & & & & \\
\hline
\end{tabular}


Table 1. Sampling frequency for volatile organic compounds and per- and polyfluoroalkyl substances in selected wells at the former Naval Air Warfare Center, West Trenton, New Jersey, 2014-17.-Continued

[bls, below land surface; VOCs, volatile organic compounds; PFASs, per- and polyfluoroalkyl substances; BR, bedrock; MW, monitoring well; S, shallow; X, indicates well was sampled]

\begin{tabular}{|c|c|c|c|c|c|c|c|c|c|c|c|c|c|c|c|c|}
\hline $\begin{array}{l}\text { Local } \\
\text { Identifier }\end{array}$ & $\begin{array}{l}\text { Depth to } \\
\text { top of open } \\
\text { interval, } \\
\text { in feet bls }\end{array}$ & $\begin{array}{l}\text { Depth to } \\
\text { bottom } \\
\text { of open } \\
\text { interval, in } \\
\text { feet bls }\end{array}$ & $\begin{array}{l}\text { Sampling } \\
\text { frequency, } \\
\text { VOCs }\end{array}$ & $\begin{array}{l}\text { VOCs } \\
\text { sam- } \\
\text { pled } \\
\text { spring } \\
2014\end{array}$ & $\begin{array}{l}\text { VOCs } \\
\text { sam- } \\
\text { pled } \\
\text { fall } \\
2014\end{array}$ & $\begin{array}{l}\text { VOCs } \\
\text { sam- } \\
\text { pled } \\
\text { spring } \\
2015\end{array}$ & $\begin{array}{l}\text { VOCs } \\
\text { sam- } \\
\text { pled } \\
\text { fall } \\
2015\end{array}$ & $\begin{array}{l}\text { VOCs } \\
\text { sam- } \\
\text { pled } \\
\text { spring } \\
2016\end{array}$ & $\begin{array}{l}\text { VOCs } \\
\text { sam- } \\
\text { pled } \\
\text { fall } \\
2016\end{array}$ & $\begin{array}{l}\text { VOCs } \\
\text { sam- } \\
\text { pled } \\
\text { spring } \\
2017\end{array}$ & $\begin{array}{l}\text { VOCs } \\
\text { sam- } \\
\text { pled } \\
\text { fall } \\
2017\end{array}$ & $\begin{array}{l}\text { PFASs } \\
\text { sam- } \\
\text { pled } \\
\text { spring } \\
2015\end{array}$ & $\begin{array}{l}\text { PFASs } \\
\text { sam- } \\
\text { pled } \\
\text { fall } \\
2016\end{array}$ & $\begin{array}{c}\text { PFASs } \\
\text { sam- } \\
\text { pled } \\
\text { spring } \\
2017\end{array}$ & $\begin{array}{c}\text { PFASs } \\
\text { sam- } \\
\text { pled } \\
\text { fall } \\
2017\end{array}$ & $\begin{array}{l}\text { 1,4-dioxane } \\
\text { sampled } \\
\text { spring } 2017\end{array}$ \\
\hline 22BR & 24 & 49 & Semiannual & $\mathrm{X}$ & $\mathrm{X}$ & $\mathrm{X}$ & $\mathrm{X}$ & $\mathrm{X}$ & $\mathrm{X}$ & $\mathrm{X}$ & $\mathrm{X}$ & & $X$ & & & $\mathrm{X}$ \\
\hline 23BR & 65 & 90 & Biennial & $\mathrm{X}$ & & & & $\mathrm{X}$ & & & & & & & & \\
\hline 24BR & 80 & 95 & Annual & $\mathrm{X}$ & & $\mathrm{X}$ & & $\mathrm{X}$ & & $\mathrm{X}$ & & & & & & \\
\hline $25 \mathrm{BR}$ & 75 & 100 & Annual & $\mathrm{X}$ & & $\mathrm{X}$ & & $\mathrm{X}$ & & $X$ & & & & & & \\
\hline 27BR & 65 & 80 & Biennial & $\mathrm{X}$ & & & & $\mathrm{X}$ & & & & & & & & \\
\hline $27 \mathrm{~S}$ & 11.2 & 21.2 & Unscheduled & & & & & & & & & & & $\mathrm{X}$ & & \\
\hline 28BR & 76 & 91 & Biennial & $\mathrm{X}$ & & & & $\mathrm{X}$ & & & & & & & & \\
\hline $28 \mathrm{~S}$ & 10 & 25 & Quadrennial & $\mathrm{X}$ & & & & & & & & & & $\mathrm{X}$ & & \\
\hline 29BR & 85 & 100 & Semiannual & $\mathrm{X}$ & $\mathrm{X}$ & $\mathrm{X}$ & $\mathrm{X}$ & $\mathrm{X}$ & $\mathrm{X}$ & $\mathrm{X}$ & $\mathrm{X}$ & & $\mathrm{X}$ & & & $\mathrm{X}$ \\
\hline $29 \mathrm{~S}$ & 10 & 20 & Quadrennial & $\mathrm{X}$ & & & & & & & & & & $\mathrm{X}$ & & \\
\hline 30BR & 85 & 110 & Biennial & $\mathrm{X}$ & & & & $\mathrm{X}$ & & & & & & & & \\
\hline $30 \mathrm{~S}$ & 7.5 & 17.5 & Quadrennial & $\mathrm{X}$ & & & & & & & & & & $\mathrm{X}$ & & \\
\hline $31 \mathrm{BR}$ & 35 & 45 & Biennial & $\mathrm{X}$ & & & & $X$ & & & & & & $X$ & & \\
\hline $31 \mathrm{~S}$ & 10 & 20 & Biennial & $\mathrm{X}$ & & & & $\mathrm{X}$ & & & & & & $X$ & & \\
\hline 32BR & 40 & 55 & Quadrennial & $\mathrm{X}$ & & & & & & & & & & $\mathrm{X}$ & & \\
\hline $32 \mathrm{~S}$ & 5 & 15 & Quadrennial & $\mathrm{X}$ & & & & & & & & & & & & \\
\hline $33 \mathrm{BR}$ & 30 & 45 & Biennial & $\mathrm{X}$ & & & & $\mathrm{X}$ & & & & & & & & \\
\hline $33 \mathrm{~S}$ & 6 & 16 & Unscheduled & & & & & & & $X$ & & & & & & \\
\hline 34BR & 35 & 48 & Quadrennial & $\mathrm{X}$ & & & & & & & & & & & & \\
\hline $34 \mathrm{~S}$ & 8 & 18 & Biennial & $\mathrm{X}$ & & & & $\mathrm{X}$ & & & & & & $\mathrm{X}$ & & $\mathrm{X}$ \\
\hline $35 \mathrm{BR}$ & 31 & 47 & Quadrennial & $\mathrm{X}$ & & & & & & & & & & & & \\
\hline 35MW1 & 7 & 25 & Biennial & $\mathrm{X}$ & & & & $\mathrm{X}$ & & & & & & & & \\
\hline 35MW2 & 6.5 & 22.5 & Biennial & $\mathrm{X}$ & & & & $\mathrm{X}$ & & & & & & & & \\
\hline $35 \mathrm{~S}$ & 5 & 15 & Quadrennial & $\mathrm{X}$ & & & & & & & & & & $\mathrm{X}$ & & \\
\hline 36BR & 100.7 & 111.9 & Annual & $\mathrm{X}$ & & $\mathrm{X}$ & & $\mathrm{X}$ & & $\mathrm{X}$ & & & & & & \\
\hline
\end{tabular}


Table 1. Sampling frequency for volatile organic compounds and per- and polyfluoroalkyl substances in selected wells at the former Naval Air Warfare Center, West Trenton, New Jersey, 2014-17.-Continued

[bls, below land surface; VOCs, volatile organic compounds; PFASs, per- and polyfluoroalkyl substances; BR, bedrock; MW, monitoring well; S, shallow; X, indicates well was sampled]

\begin{tabular}{|c|c|c|c|c|c|c|c|c|c|c|c|c|c|c|c|c|}
\hline $\begin{array}{c}\text { Local } \\
\text { Identifier }\end{array}$ & $\begin{array}{l}\text { Depth to } \\
\text { top of open } \\
\text { interval, } \\
\text { in feet bls }\end{array}$ & $\begin{array}{l}\text { Depth to } \\
\text { bottom } \\
\text { of open } \\
\text { interval, in } \\
\text { feet bls }\end{array}$ & $\begin{array}{c}\text { Sampling } \\
\text { frequency, } \\
\text { VOCs }\end{array}$ & $\begin{array}{l}\text { VOCs } \\
\text { sam- } \\
\text { pled } \\
\text { spring } \\
2014\end{array}$ & $\begin{array}{l}\text { VOCs } \\
\text { sam- } \\
\text { pled } \\
\text { fall } \\
2014\end{array}$ & $\begin{array}{l}\text { VOCs } \\
\text { sam- } \\
\text { pled } \\
\text { spring } \\
2015\end{array}$ & $\begin{array}{l}\text { VOCs } \\
\text { sam- } \\
\text { pled } \\
\text { fall } \\
2015\end{array}$ & $\begin{array}{l}\text { VOCs } \\
\text { sam- } \\
\text { pled } \\
\text { spring } \\
2016\end{array}$ & $\begin{array}{l}\text { VOCs } \\
\text { sam- } \\
\text { pled } \\
\text { fall } \\
2016\end{array}$ & $\begin{array}{l}\text { VOCs } \\
\text { sam- } \\
\text { pled } \\
\text { spring } \\
2017\end{array}$ & $\begin{array}{l}\text { VOCs } \\
\text { sam- } \\
\text { pled } \\
\text { fall } \\
2017\end{array}$ & $\begin{array}{c}\text { PFASs } \\
\text { sam- } \\
\text { pled } \\
\text { spring } \\
2015\end{array}$ & $\begin{array}{c}\text { PFASs } \\
\text { sam- } \\
\text { pled } \\
\text { fall } \\
2016\end{array}$ & $\begin{array}{l}\text { PFASs } \\
\text { sam- } \\
\text { pled } \\
\text { spring } \\
2017\end{array}$ & $\begin{array}{c}\text { PFASs } \\
\text { sam- } \\
\text { pled } \\
\text { fall } \\
2017\end{array}$ & $\begin{array}{l}\text { 1,4-dioxane } \\
\text { sampled } \\
\text { spring } 2017\end{array}$ \\
\hline $37 \mathrm{BR}$ & 60 & 75 & Quadrennial & $X$ & & & & & & & & & & $\mathrm{X}$ & & $\mathrm{X}$ \\
\hline $37 \mathrm{~S}$ & 6 & 16 & Biennial & $\mathrm{X}$ & & & & $\mathrm{X}$ & & & & & & $X$ & & \\
\hline $38 \mathrm{BR}$ & 100 & 115 & Biennial & $\mathrm{X}$ & & & & $\mathrm{X}$ & & & & & & & & \\
\hline 39BR & 68 & 88 & Biennial & $\mathrm{X}$ & & & & $\mathrm{X}$ & & & & & & & & \\
\hline 40BR & 95 & 120 & Annual & $\mathrm{X}$ & & $\mathrm{X}$ & & $\mathrm{X}$ & & $\mathrm{X}$ & & & & & & \\
\hline $40 \mathrm{~S}$ & 3 & 13 & Annual & $X$ & & $\mathrm{X}$ & & $\mathrm{X}$ & & $\mathrm{X}$ & & & & & & \\
\hline $41 \mathrm{BR}$ & 85 & 110 & Biennial & $\mathrm{X}$ & & & & $\mathrm{X}$ & & & & & & & & \\
\hline 42BR & 120 & 140 & Biennial & $\mathrm{X}$ & & & & $\mathrm{X}$ & & & & & & & & \\
\hline $42 \mathrm{~S}$ & 3.6 & 13.6 & Quadrennial & $\mathrm{X}$ & & & & & & & & & & $X$ & & \\
\hline $43 \mathrm{BR}$ & 385 & 410 & Quadrennial & $\mathrm{X}$ & & & & & & & & & & & & \\
\hline 44BR & 305 & 330 & Quadrennial & $\mathrm{X}$ & & & & & & & & & & & & \\
\hline $45 \mathrm{BR}$ & 185 & 210 & Semiannual & $\mathrm{X}$ & $X$ & $\mathrm{X}$ & $\mathrm{X}$ & $\mathrm{X}$ & $\mathrm{X}$ & $X$ & $\mathrm{X}$ & & $\mathrm{X}$ & & & $\mathrm{X}$ \\
\hline 46BR & 196 & 221 & Annual & $X$ & & $X$ & & $\mathrm{X}$ & & $\mathrm{X}$ & & & & & & \\
\hline $47 \mathrm{BR}$ & 3 & 18 & Annual & $\mathrm{X}$ & & $X$ & & $\mathrm{X}$ & & $\mathrm{X}$ & & & & & & \\
\hline $48 \mathrm{BR}$ & 82 & 100 & Semiannual & $\mathrm{X}$ & $X$ & $\mathrm{X}$ & & $\mathrm{X}$ & $\mathrm{X}$ & $\mathrm{X}$ & $\mathrm{X}$ & $\mathrm{X}$ & $\mathrm{X}$ & $\mathrm{X}$ & & $\mathrm{X}$ \\
\hline 49BR & 42 & 60 & Biennial & $\mathrm{X}$ & & & & $\mathrm{X}$ & & & & & & & & \\
\hline $50 \mathrm{BR}$ & 60 & 80 & Biennial & $X$ & & & & $\mathrm{X}$ & & & & & & $\mathrm{X}$ & & \\
\hline $51 \mathrm{BR}$ & 86 & 96 & Biennial & $\mathrm{X}$ & & & & $\mathrm{X}$ & & & & & & $\mathrm{X}$ & & \\
\hline $52 \mathrm{BR}$ & 155 & 180 & Biennial & $\mathrm{X}$ & & & & $\mathrm{X}$ & & & & & & & & \\
\hline $53 \mathrm{BR}$ & 95 & 120 & Biennial & $\mathrm{X}$ & & & & $\mathrm{X}$ & & & & & & & & \\
\hline $54 \mathrm{BR}$ & 175 & 200 & Annual & $\mathrm{X}$ & & $\mathrm{X}$ & & $\mathrm{X}$ & & $X$ & & & & $X$ & & $\mathrm{X}$ \\
\hline $55 \mathrm{BR}$ & 135 & 160 & Biennial & $X$ & & & & $\mathrm{X}$ & & & & & & & & \\
\hline $56 \mathrm{BR}$ & 140 & 165 & Semiannual & $X$ & & $X$ & $\mathrm{X}$ & $\mathrm{X}$ & $\mathrm{X}$ & $X$ & $X$ & & X & & & $X$ \\
\hline $57 \mathrm{BR}$ & 12 & 27 & Quadrennial & $\mathrm{X}$ & & & & & & & & & & & & \\
\hline $58 \mathrm{BR}$ & 85 & 110 & Quadrennial & $\mathrm{X}$ & & & & & & & & & & & & \\
\hline
\end{tabular}


Table 1. Sampling frequency for volatile organic compounds and per- and polyfluoroalkyl substances in selected wells at the former Naval Air Warfare Center, West Trenton, New Jersey, 2014-17.-Continued

[bls, below land surface; VOCs, volatile organic compounds; PFASs, per- and polyfluoroalkyl substances; BR, bedrock; MW, monitoring well; S, shallow; X, indicates well was sampled]

\begin{tabular}{|c|c|c|c|c|c|c|c|c|c|c|c|c|c|c|c|c|}
\hline $\begin{array}{l}\text { Local } \\
\text { Identifier }\end{array}$ & $\begin{array}{l}\text { Depth to } \\
\text { top of open } \\
\text { interval, } \\
\text { in feet bls }\end{array}$ & $\begin{array}{l}\text { Depth to } \\
\text { bottom } \\
\text { of open } \\
\text { interval, in } \\
\text { feet bls }\end{array}$ & $\begin{array}{l}\text { Sampling } \\
\text { frequency, } \\
\text { VOCs }\end{array}$ & $\begin{array}{l}\text { VOCs } \\
\text { sam- } \\
\text { pled } \\
\text { spring } \\
2014\end{array}$ & $\begin{array}{l}\text { VOCs } \\
\text { sam- } \\
\text { pled } \\
\text { fall } \\
2014\end{array}$ & $\begin{array}{l}\text { VOCs } \\
\text { sam- } \\
\text { pled } \\
\text { spring } \\
2015\end{array}$ & $\begin{array}{l}\text { VOCs } \\
\text { sam- } \\
\text { pled } \\
\text { fall } \\
2015\end{array}$ & $\begin{array}{l}\text { VOCs } \\
\text { sam- } \\
\text { pled } \\
\text { spring } \\
2016\end{array}$ & $\begin{array}{l}\text { VOCs } \\
\text { sam- } \\
\text { pled } \\
\text { fall } \\
2016\end{array}$ & $\begin{array}{l}\text { VOCs } \\
\text { sam- } \\
\text { pled } \\
\text { spring } \\
2017\end{array}$ & $\begin{array}{l}\text { VOCs } \\
\text { sam- } \\
\text { pled } \\
\text { fall } \\
2017\end{array}$ & $\begin{array}{l}\text { PFASs } \\
\text { sam- } \\
\text { pled } \\
\text { spring } \\
2015\end{array}$ & $\begin{array}{l}\text { PFASs } \\
\text { sam- } \\
\text { pled } \\
\text { fall } \\
2016\end{array}$ & $\begin{array}{l}\text { PFASs } \\
\text { sam- } \\
\text { pled } \\
\text { spring } \\
2017\end{array}$ & $\begin{array}{l}\text { PFASs } \\
\text { sam- } \\
\text { pled } \\
\text { fall } \\
2017\end{array}$ & $\begin{array}{l}\text { 1,4-dioxane } \\
\text { sampled } \\
\text { spring } 2017\end{array}$ \\
\hline $59 \mathrm{BR}$ & 56 & 80 & Quadrennial & $\mathrm{X}$ & & & & & & & & & & & & $\mathrm{X}$ \\
\hline $60 \mathrm{BR}$ & 70 & 85 & Annual & $\mathrm{X}$ & & $\mathrm{X}$ & & $\mathrm{X}$ & & $\mathrm{X}$ & & & & & & $\mathrm{X}$ \\
\hline 61BR & 70 & 100 & Annual & $\mathrm{X}$ & & $\mathrm{X}$ & & $\mathrm{X}$ & & & & & & & & \\
\hline $62 \mathrm{BR}$ & 142 & 167 & Quadrennial & $\mathrm{X}$ & & & & & & & & & & & & \\
\hline $63 \mathrm{BR}$ & 15 & 40 & Biennial & $\mathrm{X}$ & & & & $\mathrm{X}$ & & & & & & & & \\
\hline 64BR & 15 & 40 & Biennial & $\mathrm{X}$ & & & & $\mathrm{X}$ & & & & & & & & \\
\hline $65 \mathrm{BR}$ & 15 & 40 & Annual & $\mathrm{X}$ & & $X$ & & $\mathrm{X}$ & & $\mathrm{X}$ & & & & & & \\
\hline $66 \mathrm{BR}$ & 70 & 100 & Quadrennial & $\mathrm{X}$ & & & & & & & & & & & & \\
\hline 70BR-10 & 6 & 16 & Quadrennial & $\mathrm{X}$ & & & & $\mathrm{X}$ & & & & & & & & \\
\hline 70BR-72 & 68.5 & 73.5 & Unscheduled & & & $X$ & & & & & & & & & & \\
\hline 71BR-A & 25 & 50.2 & Quadrennial & $\mathrm{X}$ & & & & $X$ & & & & & & & & \\
\hline 73BR-A & 25 & 34.6 & Quadrennial & $\mathrm{X}$ & & & & $X$ & & & & & & & & \\
\hline 74BR & 52 & 77 & Quadrennial & $\mathrm{X}$ & & & & & & & & & & & & \\
\hline 80BR-A & 67.3 & 78.5 & Unscheduled & & & X & & & & & & & & & & \\
\hline $91 \mathrm{BR}$ & 7 & 17 & Semiannual & X & & X & $X$ & X & X & X & X & & $X$ & & & $X$ \\
\hline BRP01 & 20 & 60 & Biennial & $\mathrm{X}$ & & & & $\mathrm{X}$ & & & & & & & & \\
\hline BRP02 & 25 & 45 & Semiannual & $\mathrm{X}$ & & $\mathrm{X}$ & $\mathrm{X}$ & $\mathrm{X}$ & $\mathrm{X}$ & $\mathrm{X}$ & $\mathrm{X}$ & & $\mathrm{X}$ & & & $\mathrm{X}$ \\
\hline BRP03 & 25 & 45 & Biennial & $\mathrm{X}$ & & & & $X$ & & & & & & & & \\
\hline WDW & 10.3 & 10.3 & Semiannual & $X$ & & $X$ & $X$ & & & & & & & & & \\
\hline Totals & & & & 88 & 7 & 27 & 10 & 64 & 10 & 24 & 10 & 1 & 18 & 26 & 0 & 19 \\
\hline
\end{tabular}


Samples were also collected on a test basis from five wells for PFAS compounds using RCDM passive samplers. RCDM passive samplers were constructed, deployed, and sampled using the procedures detailed in Imbrigiotta and others (2008) and Imbrigiotta and Harte (2020). The RCDM samplers were equilibrated in the wells for 3 weeks prior to retrieval and sampling. Immediately after the passive samplers were retrieved, the intake line for the peristaltic pump was lowered to the same depth where the RCDM sampler had been deployed. The well was low-flow purged, field physical and chemical characteristics were monitored to stability, and samples were collected and sent to the same laboratory for analysis of PFASs. An equipment blank from the low-flow purge pump and an equipment blank from an RCDM sampler that was stored for 3 weeks in the same water used to fill the RCDM samplers were also collected and sent to the same laboratory for analysis of PFASs.

\section{Analytical Methods}

All VOC, PFAS, and 1,4-dioxane samples collected by the Navy's contractors were sent to a Navy and NJDEP certified laboratory for analysis. VOC analyses were run using EPA Method 8260 (EPA, 2018a). PFAS analyses were run using a modification of EPA Method 537 (EPA, 2018b). 1,4-dioxane analyses were run using EPA Method 8270E (EPA, 2018c). Analytical results were sent to the contractors for quality assurance/quality control review. The approved data were forwarded, with permission from the Navy, to the USGS.

\section{Results and Discussion}

The water-quality data collected during 2015, 2016, and 2017 were primarily for VOCs (Watermark Environmental, 2016b; 2017; Tetra Tech, 2018a). To evaluate changes in VOC concentrations over the 2015-17 period, results from these years were compared to the results from the 2014 comprehensive sampling (Watermark, 2015b). All VOC data discussed in this report are included in Appendix 2 and a USGS data release (Fiore and Imbrigiotta, 2020).

The areal and vertical extents of the TCE, cisDCE, and VC contamination were plotted on maps and cross sections using color-coded dots to indicate the degree of contamination. A green dot over a well or open interval indicates that the concentration of the VOC was less than $(<) 1 \mu \mathrm{g} / \mathrm{L}$. A yellow dot over a well or open interval indicates that the concentration of the VOC was 1 to $<100 \mu \mathrm{g} / \mathrm{L}$. An orange dot over a well or open interval indicates that the concentration of the VOC was 100 to $<1,000 \mu \mathrm{g} / \mathrm{L}$. A red dot over a well or open interval indicates that the concentration of the VOC was greater than or equal to $(\geq) 1,000 \mu \mathrm{g} / \mathrm{L}$. If a well was not sampled during a year, the well is represented as a small black dot on the map or an uncovered open interval on the cross section. The data used to define the dots plotted on these maps and cross sections are listed in Appendix 2. Areas of VOC contamination on the maps and cross sections where the wells have concentrations of VOCs $\geq 1 \mu \mathrm{g} / \mathrm{L}$ are approximated and enclosed by a dashed line. In years when a larger number of samples were collected (2014 and 2016), these VOC contamination areas are shaded in pink. The maps do not differentiate between contamination at different depths in the aquifer. The cross sections show the variation in contamination with depth. VOC data with "less than" values were plotted at one-half that value on the maps and cross sections. The variation in concentrations of TCE, cisDCE, and VC over time was plotted on graphs. Historical data prior to 2014 were included on these graphs, if the data were available.

PFAS data were collected only during 2015, 2016, and 2017 (Watermark Environmental, 2016b; 2017; Tetra Tech, 2018b). All PFAS data discussed in this report are included in Appendix 2.

The areal and vertical extents of the PFOS, PFOA, and PFNA contamination also were plotted on maps and cross sections using color-coded dots to indicate the degree of contamination. A green dot over a well or open interval indicates that the concentrations of PFOS and PFNA were $<13 \mathrm{ng} / \mathrm{L}$ and the concentration of PFOA was $<14 \mathrm{ng} / \mathrm{L}$. A yellow dot over a well or open interval indicates that the concentrations of PFOS and PFOA were from 13 or 14 to $<70 \mathrm{ng} / \mathrm{L}$, and the concentration of PFNA was from 13 to $<100 \mathrm{ng} / \mathrm{L}$. An orange dot over a well or open interval indicates that the concentrations of PFOS and PFOA were from 70 to $<1000 \mathrm{ng} / \mathrm{L}$ and the concentrations of PFNA were from 100 to $<1,000 \mathrm{ng} / \mathrm{L}$. A red dot over a well or open interval indicates that the concentrations of PFOS, PFOA, and PFNA were $\geq 1,000 \mathrm{ng} / \mathrm{L}$. If a well was not sampled during a year, the well is represented as a small black dot on the map or an uncovered open interval on the cross section. The data used to define the dots plotted on these maps and cross sections are listed in Appendix 2. Areas of PFAS contamination on the maps and cross sections where the wells have concentrations of PFOS and PFNA $>13 \mathrm{ng} / \mathrm{L}$ or PFOA $>14 \mathrm{ng} / \mathrm{L}$ are defined by the distribution of yellow, orange, and red dots.. The maps do not differentiate between contamination at different depths in the aquifer. The cross sections show the variation in PFAS concentrations with depth. Data with "less than" values were plotted at one-half that value on the maps and cross sections.

\section{Areal Extent of VOC Contamination at NAWC During 2014-17}

Data for TCE, cisDCE, and VC are presented in sets of 4 maps, 1 for each year from 2014 through 2017. All maps generally show two areas of VOC concentrations $\geq 1 \mu \mathrm{g} / \mathrm{L}$. The larger area of contamination was always at the Site 1 area on the western side of the former NAWC property, which included the main source of TCE contamination between Buildings 40 and 41 (fig. 1). The smaller area of contamination was always at the Site 3 area on the eastern side of the 
former NAWC property, which included the TCE source areas of a former wastewater lagoon and a sludge drying and disposal area (fig. 1).

\section{Areal Extent of TCE Contamination During 2014-17}

Of the 91 wells sampled for TCE at NAWC from 2014 to 2017,53 wells had concentrations of TCE $<1 \mu \mathrm{g} / \mathrm{L}$, and 38 wells had concentrations of TCE $\geq 1 \mu \mathrm{g} / \mathrm{L}$ in their most recent sampling. Between 2014 and their most recent sampling, 70 wells had either decreased or unchanged TCE concentrations. Of the 21 wells that had an increase in TCE concentration over this time, 14 had increases of $<10 \mu \mathrm{g} / \mathrm{L}$ or less than 30 percent and could be considered essentially unchanged. The other seven wells had substantial increases in TCE concentration of more than $700 \mu \mathrm{g} / \mathrm{L}$ or more than 30 percent. These wells are discussed below.

TCE concentrations were plotted areally for each year on figures 4-7. In the Site 1 area on the western side of NAWC, in 2014, the TCE contamination covered an area approximately bounded by the former Administration Building (Building 21) on the east, Parkway Avenue on the south, Jack Stephan Way on the west, and the airport property boundary on the north (fig. 4). The shaded area was slightly smaller in 2016 (fig. 6) because TCE concentrations in two wells (35MW2 and 21BR) in the northwestern corner of the site decreased from slightly $>1 \mu \mathrm{g} / \mathrm{L}$ to slightly $<1 \mu \mathrm{g} / \mathrm{L}$. The areal extents of TCE contamination in 2015 (fig. 5) and 2017 (fig. 7) were estimated based on the smaller number of wells sampled during these two years and data from the years before and after. Therefore, any decreases in the size of contamination areas in 2015 and 2017 were not because of any natural or engineered remediation.

From 2014 to 2017, a set of nine wells consistently had TCE concentrations $\geq 1,000 \mu \mathrm{g} / \mathrm{L}$ (red dots) in the Site 1 area. Most of these wells were in the central part of the western shaded area of figures 4-7. Wells 24BR (20,000-29,000 $\mu \mathrm{g} / \mathrm{L})$ and 56BR $(2,600-14,000 \mu \mathrm{g} / \mathrm{L})$ were the wells with the highest TCE concentrations during this time. For the most part, the wells that were highly contaminated with TCE were in the areas where most of the TCE was spilled, between Buildings 40 and 41 or downdip from these areas. It is worthwhile to note that almost all the highly contaminated wells (red dots) remained so over this 4-year period, indicating that TCE concentrations were not declining very quickly over time. This will be further illustrated later in the "Variation in VOC Contamination over Time" section where the graphs of concentration in relation to time are presented.

All seven wells that showed substantial increases in TCE concentration from 2014 to 2017 were in the Site 1 area. Wells 24BR, 29BR, 45BR, 70BR-10, 71BR-A, 73BR-A, and BRP02 all increased by $\geq 700 \mu \mathrm{g} / \mathrm{L}$ over this period. Wells 29BR, 45BR, and BRP02 are all extraction wells for the pump-and-treat plant, so the increase in TCE concentrations in these wells may be due to drawing in more highly contaminated groundwater. The increases in wells 24BR, 70BR-10, 71BR-A, 73BR-A may be due to continued downward movement of dissolving DNAPL TCE contamination in the Site 1 area under the influence of recharge and the pumping of the extraction well system.

There were no offsite wells to the west or south of the NAWC site that contained TCE concentrations $\geq 1 \mu \mathrm{g} / \mathrm{L}$, even though the unstressed groundwater flow direction was generally toward the southwest. This was a good indication that the TCE contamination was being hydraulically contained by the pump-and-treat system in the western Site 1 area.

The TCE contamination in the Site 3 area on the eastern side of the NAWC site was less extensive than the area of TCE contamination in the Site 1 area on the western side of the NAWC site over the 2014-17 period. The TCE concentrations in wells in Site 3 area were generally 1-2 orders of magnitude lower than most of the TCE concentrations in wells in the Site 1 area. In 2014, the Site 3 area of TCE contamination appeared to originate near the former wastewater lagoon and sludge drying beds (fig. 4). In 2016 the Site 3 contamination area was smaller because concentrations of TCE in wells $11 \mathrm{BR}$ and $51 \mathrm{BR}$ decreased from slightly $>1 \mu \mathrm{g} / \mathrm{L}$ to slightly $<1 \mu \mathrm{g} / \mathrm{L}$ (fig. 6). In 2015 and 2017 the likely areas of TCE contamination were estimated based on the smaller number of wells sampled during these two years and data from the years before and after (figs. 5 and 7). Well 48BR, a pump-and-treat extraction well, continued to be the most contaminated well in the Site 3 area ranging in TCE concentration from 490 to 570 $\mu \mathrm{g} / \mathrm{L}$ from 2014 to 2017 . This increase in TCE concentration may be due to the well drawing in more highly contaminated groundwater over time. Well 50BR, a monitoring well east of the railroad tracks (the current eastern boundary of the NAWC site), had TCE concentrations of 3.3-3.7 $\mu \mathrm{g} / \mathrm{L}$ during this time, indicating that there was some low-level TCE-contaminated groundwater flowing off the site to the northeast. There were also two wells south of the fault (31BR and 54BR) in the eastern part of the NAWC site that were contaminated with TCE, indicating that the fault and the pump-and-treat system were not completely preventing southward or eastward groundwater flow in the bedrock.

\section{Areal Extent of cisDCE Contamination During 2014-17}

Of the 91 wells sampled for cisDCE at NAWC from 2014 to 2017,46 wells had concentrations of cisDCE $<1$ $\mu \mathrm{g} / \mathrm{L}$, and 45 wells had concentrations of cisDCE $\geq 1 \mu \mathrm{g} / \mathrm{L}$ in their most recent sampling. Between 2014 and their most recent samplings, 74 wells had either decreased or unchanged cisDCE concentrations. Of the 17 wells that had increases in cisDCE concentration over this period, 6 increased $<10 \mu \mathrm{g} / \mathrm{L}$ or less than 30 percent and could be considered essentially 


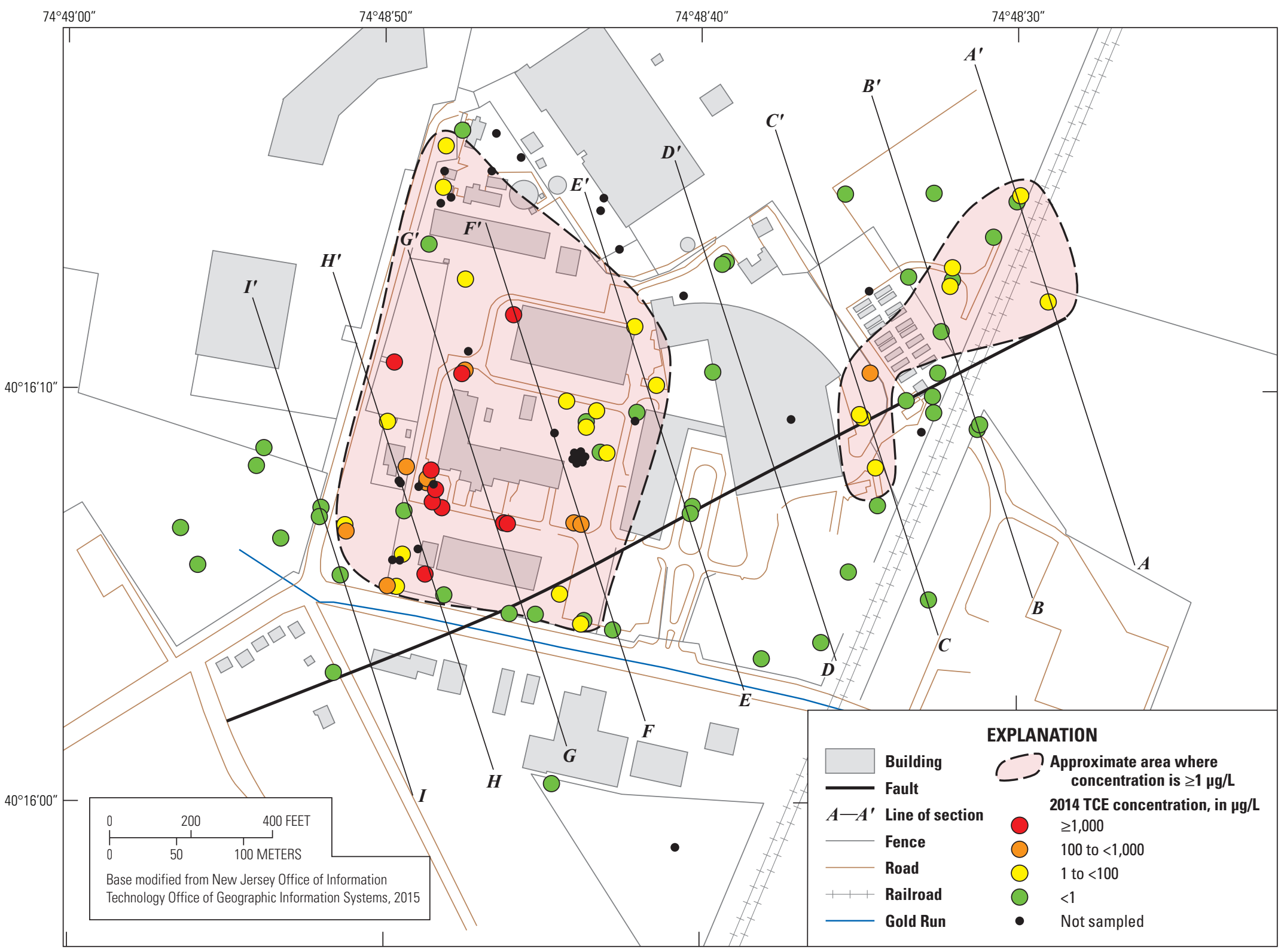

Figure 4. Areal extent of trichloroethene contamination in wells at the former Naval Air Warfare Center, West Trenton, New Jersey, 2014. (TCE, trichloroethene; mg/L, micrograms per liter) 


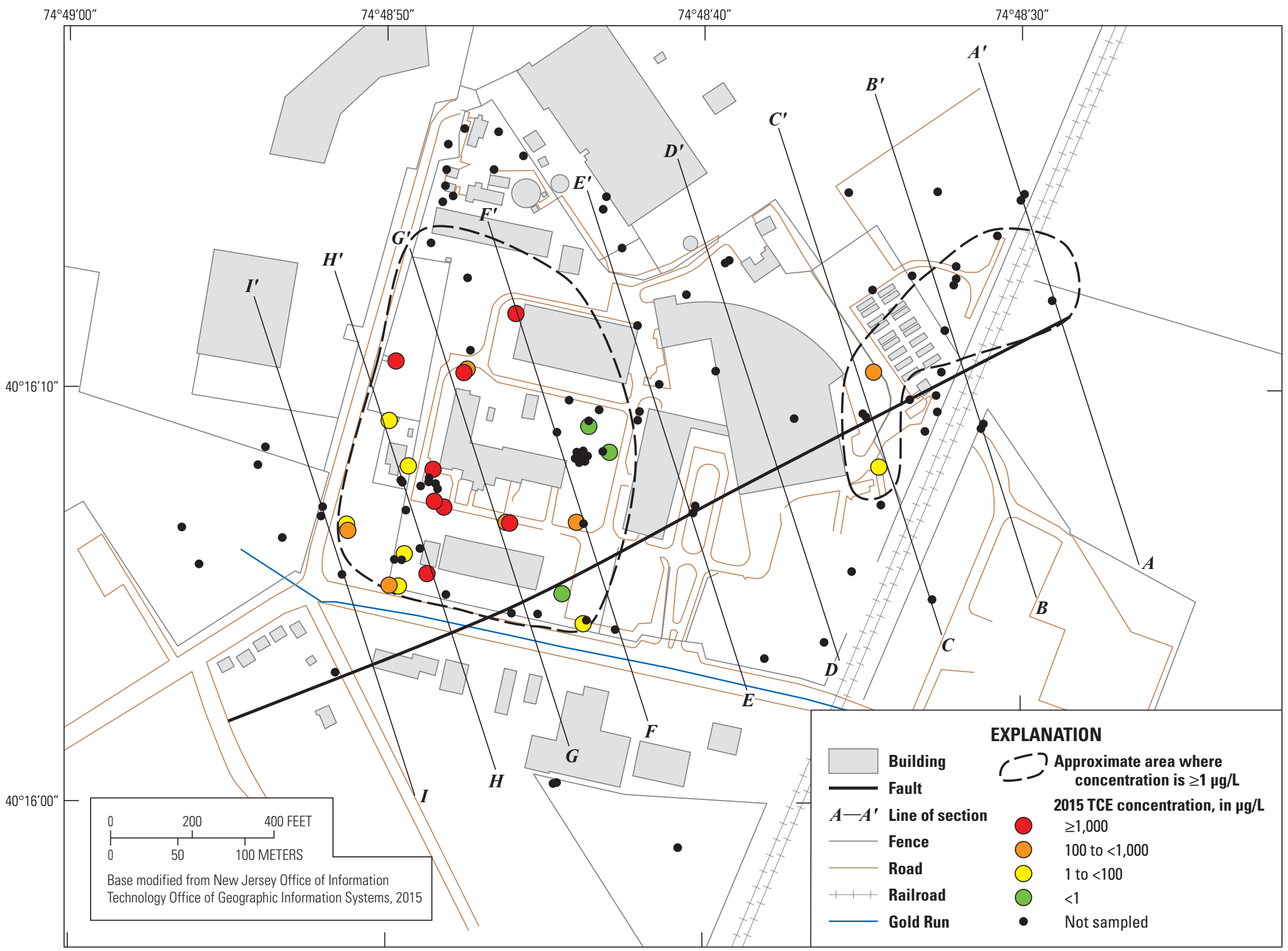

Figure 5. Areal extent of trichloroethene contamination in wells at the former Naval Air Warfare Center, West Trenton, New Jersey, 2015. (TCE, trichloroethene; mg/L, micrograms per liter) 


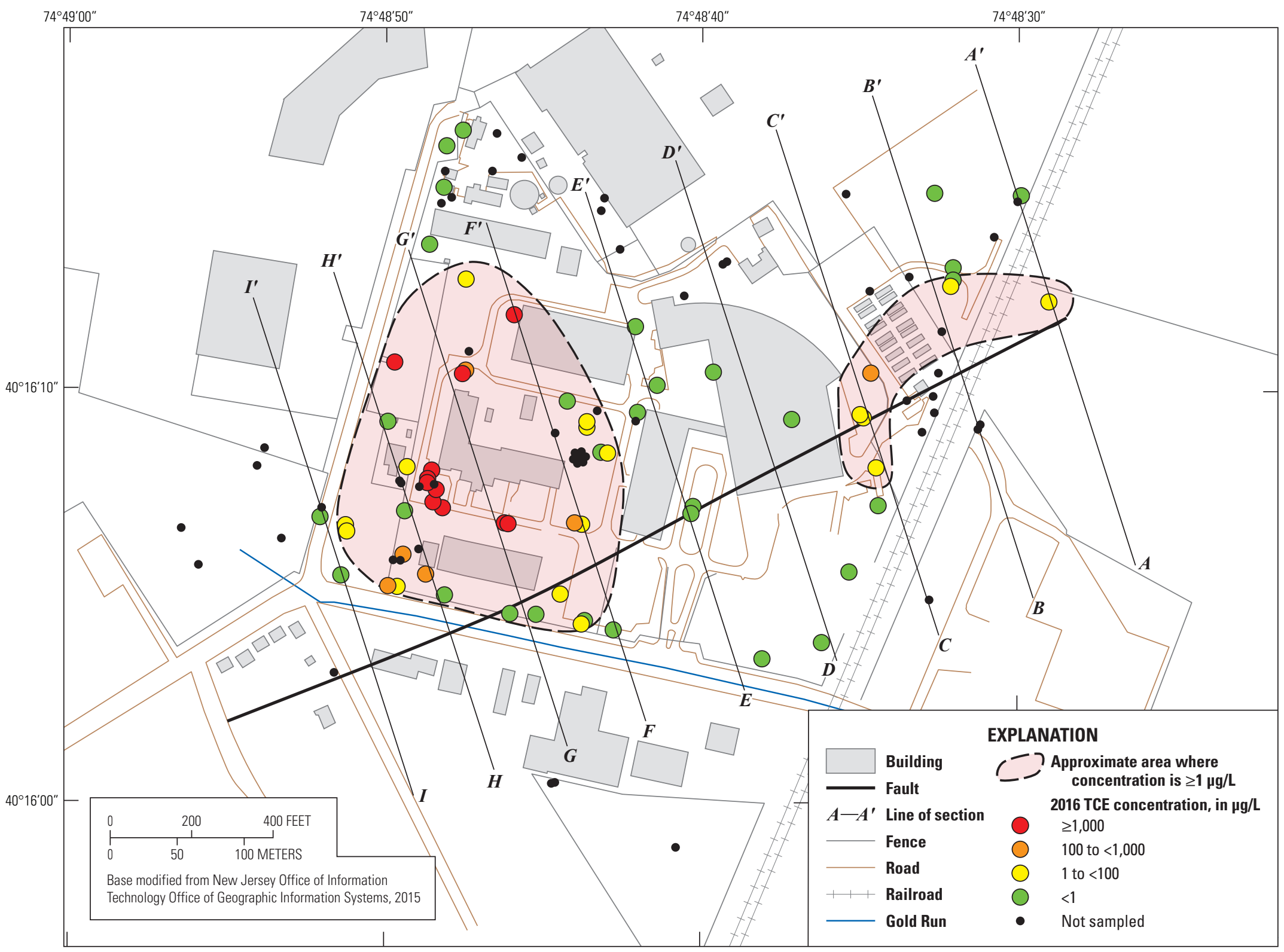

Figure 6. Areal extent of trichloroethene contamination in wells at the former Naval Air Warfare Center, West Trenton, New Jersey, 2016. (TCE, trichloroethene; mg/L, micrograms per liter) 


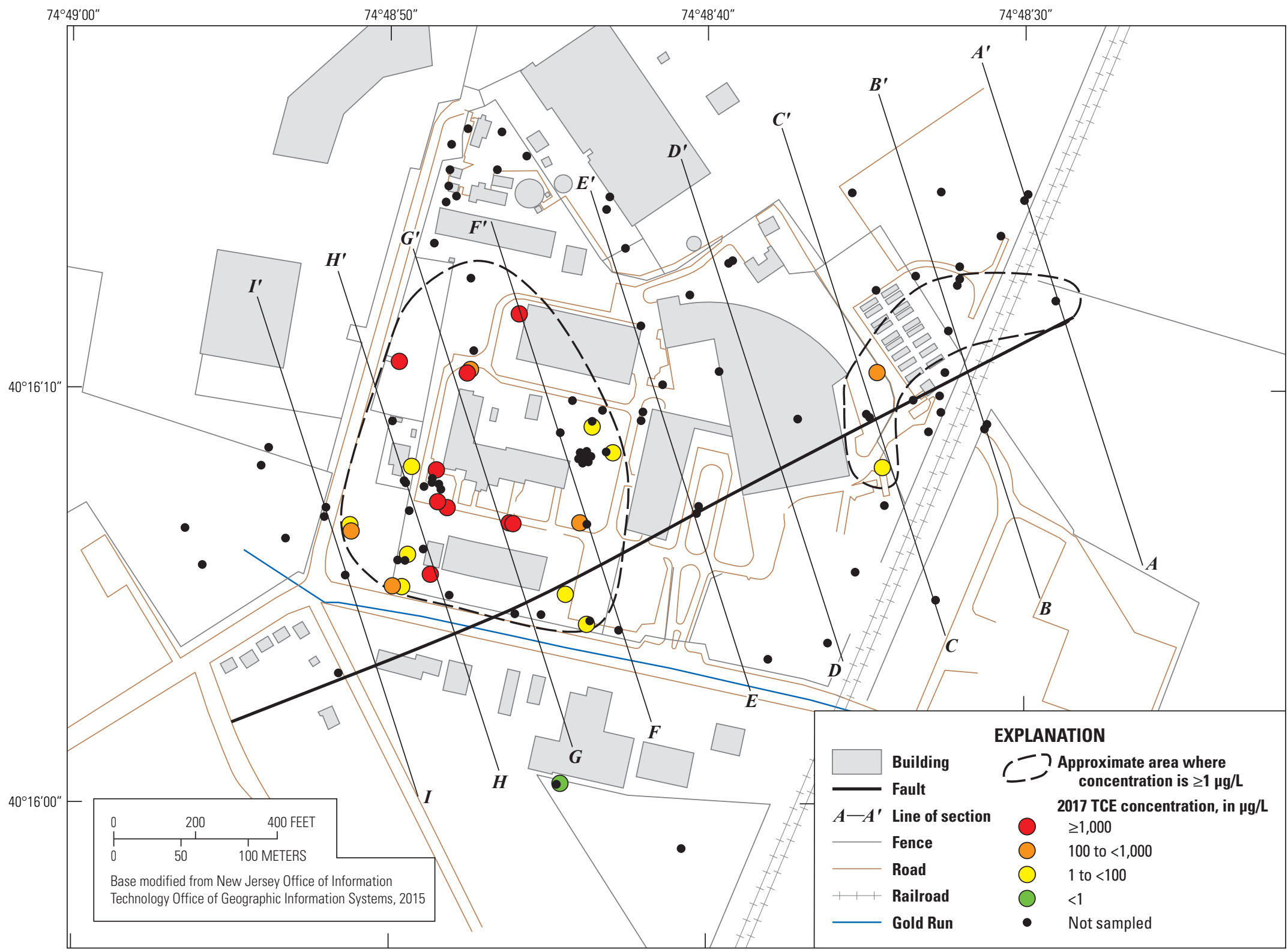

Figure 7. Areal extent of trichloroethene contamination in wells at the former Naval Air Warfare Center, West Trenton, New Jersey, 2017. (TCE, trichloroethene; mg/L, micrograms per liter) 
unchanged. The other 11 wells increased in cisDCE concentration by more than $58 \mu \mathrm{g} / \mathrm{L}$ or more than 30 percent and are discussed below.

The cisDCE concentrations are plotted in figures 8-11. In 2014 in the western Site 1 area (fig. 8), the shaded area of cisDCE contamination was approximately the same size as the TCE-contaminated area (fig. 4). In most cases, cisDCE concentrations were detected only in wells where TCE concentrations were detected. This is consistent with TCE being the contaminant spilled and cisDCE being its primary anaerobic biodegradation product. The Site 1 shaded area of cisDCE contamination was essentially identical in 2014 and 2016 (figs. 8 and 10). The likely areal extents of cisDCE contamination in 2015 (fig. 9) and 2017 (fig. 11) were estimated based on the smaller number of wells sampled during these two years and data from years before and after. Therefore, any decreases in the size of contamination areas in 2015 and 2017 were not because of any natural or engineered remediation.

From 2014 to 2017, a set of 14 wells had cisDCE concentrations $\geq 1,000 \mu \mathrm{g} / \mathrm{L}$ (red dots) in the Site 1 area. Most of these wells were in the center of the shaded area (figs. 8-11). Wells 36BR $(11,000 \mu \mathrm{g} / \mathrm{L}-56,000 \mu \mathrm{g} / \mathrm{L})$, BRP02 (73,000-12,000 $\mu \mathrm{g} / \mathrm{L})$, and $07 \mathrm{BR}(7,300-10,000 \mu \mathrm{g} / \mathrm{L})$ had the highest cisDCE concentrations during the period. The cisDCE concentrations were high in 36BR because it was the injection well for the 2008 bioaugmentation study that received a bacterial consortia and ESO (Shapiro and others, 2018) (fig. 2). The cisDCE concentrations were high in BRP02 because this well is in the area where jet fuel was stored and spilled, which provided a food source that stimulated degradation of TCE by the indigenous bacteria in the bedrock aquifer (fig. 2). Like the TCE areal distribution, almost all the highly contaminated wells (red dots) remained so over the 4-year period, indicating that cisDCE concentrations were not declining very quickly over time. Also, like the TCE areal distribution, cisDCE contamination was not found in wells to the west and south of the NAWC site.

All 11 wells that showed substantial increases in cisDCE concentration from 2014 to 2017 were in the Site 1 area. Concentrations of cisDCE in wells 07BR, 24BR, 25BR, 70BR-10, 71BR-A, 70BR-72, and 73BR-A increased by $\geq 1,300 \mu \mathrm{g} / \mathrm{L}$ over the study period. Wells $25 \mathrm{BR}, 70 \mathrm{BR}-10$, 71BR-A, 70BR-72, and 73BR-A are in the area of the 2008 bioaugmentation study, so their increased cisDCE concentrations may be due to the more favorable geochemical conditions created by the study in the bedrock aquifer. The smaller increases in wells 29BR and BRP02 probably are the result of pump-and-treat wells drawing in groundwater with higher cisDCE concentrations over the period.

In the Site 3 area on the eastern side of the NAWC site, the area of cisDCE contamination was smaller than the area of cisDCE contamination in the Site 1 area on the western side of the NAWC site from 2014 to 2017. The cisDCE concentrations in wells in the Site 3 area were generally 1-2 orders of magnitude lower than most of the cisDCE concentrations in wells in the Site 1 area. In 2014 (fig. 8), the Site 3 area of
cisDCE contamination appeared to originate near the former wastewater lagoon and sludge drying beds (fig. 1). In 2016, the cisDCE contamination area was slightly smaller than in 2014 because the concentration in well 11BR decreased from slightly $>1 \mu \mathrm{g} / \mathrm{L}$ to slightly $<1 \mu \mathrm{g} / \mathrm{L}$ (fig. 10 ). The likely areal extents of cisDCE contamination in 2015 (fig. 9) and 2017 (fig. 11) were estimated based on the smaller number of wells sampled during these two years and data from years before and after. Well 48BR, the pump-and-treat extraction well on the eastern side of the facility, was one of the most contaminated wells in this area with cisDCE concentrations ranging from 18 to $33 \mu \mathrm{g} / \mathrm{L}$ from 2014 to 2017 . Well 50BR, a monitoring well east of the railroad tracks, had cisDCE concentrations of $2.2-2.6 \mu \mathrm{g} / \mathrm{L}$ during the study period. These detected concentrations indicate that there was some flow of low-level cisDCE-contaminated groundwater off the site to the northeast and that the pump-and-treat system was not fully containing the contaminant plume on the eastern side of the site.

\section{Areal Extent of VC Contamination During 2014-17}

Of the 91 wells sampled for VC at NAWC from 2014 to 2017, 64 wells had concentrations of $\mathrm{VC}<1 \mu \mathrm{g} / \mathrm{L}$, and 27 wells had concentrations of $\mathrm{VC} \geq 1 \mu \mathrm{g} / \mathrm{L}$ in their most recent sampling. Between 2014 and their most recent sampling, 79 wells had either decreased or unchanged VC concentrations. Of the other 12 wells that experienced increases in $\mathrm{VC}$ concentration over this period, 7 increased $<10 \mu \mathrm{g} / \mathrm{L}$ or less than 30 percent and could be considered essentially unchanged. The other 5 wells increased in VC concentration by more than $26 \mu \mathrm{g} / \mathrm{L}$ or more than 30 percent and are discussed below.

The VC concentrations are plotted in figures $12-15$. In 2014 (fig. 12), the Site 1 shaded area of VC contamination was smaller in size than the TCE- or cisDCE-contaminated areas (figs. 4 and 8). In most cases, $\mathrm{VC}$ concentrations were detected only in wells where TCE and cisDCE concentrations also were detected. This is consistent with TCE being the contaminant spilled and cisDCE and VC being its primary anaerobic biodegradation products. The Site 1 shaded area of $2016 \mathrm{VC}$ contamination (fig. 14) was similar in size to that of 2014 (fig. 12). The likely areal extents of VC contamination in 2015 (fig. 13) and 2017 (fig. 15) in the Site 1 area were estimated based on the smaller number of wells sampled during these two years and data from the years before and after. Therefore, any decreases in size of contamination areas in 2015 and 2017 were not because of any natural or engineered remediation. Like the TCE and cisDCE areal distributions, VC contamination of $\geq 1 \mu \mathrm{g} / \mathrm{L}$ was not found in wells off the NAWC site to the west and south.

From 2014 to 2017, only three wells had VC concentrations $\geq 1,000 \mu \mathrm{g} / \mathrm{L}$ (red dots). Wells 36BR $(4,500-18,000$ $\mu \mathrm{g} / \mathrm{L}), \mathrm{BRP} 02(2,300-3,200 \mu \mathrm{g} / \mathrm{L})$, and 07BR (510 -1,200 $\mathrm{mg} / \mathrm{L}$ ) had the highest $\mathrm{VC}$ concentrations during 2014-17. The VC concentrations were high in 36BR because it was the injection well for the 2008 bioaugmentation study that received a bacterial consortia and ESO (Shapiro and others, 


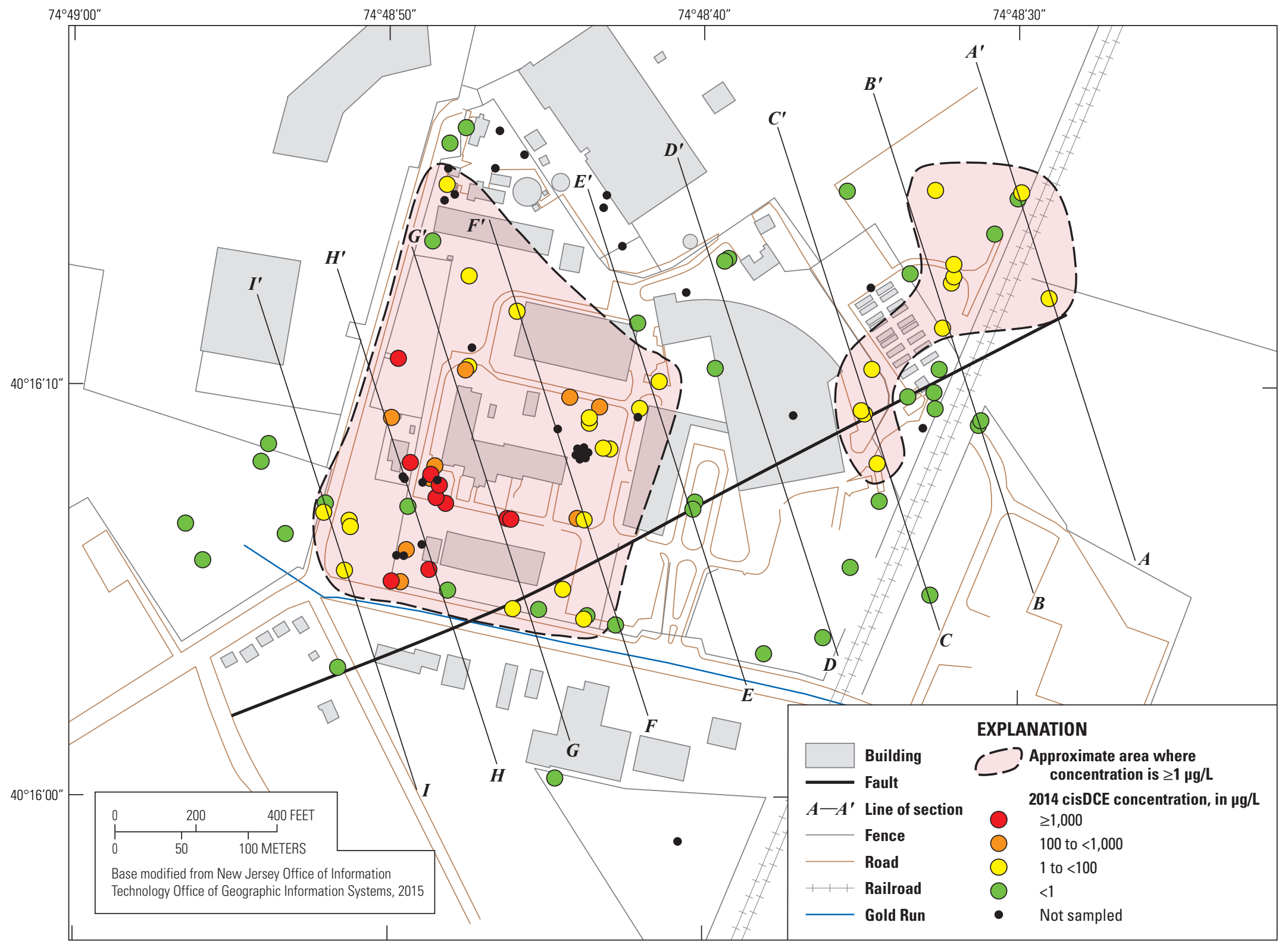

Figure 8. Areal extent of cis-1,2-dichloroethene contamination in wells at the former Naval Air Warfare Center, West Trenton, New Jersey, 2014. (cisDCE, cis-1,2-dichloroethene; $\mathrm{mg} / \mathrm{L}$, micrograms per liter) 


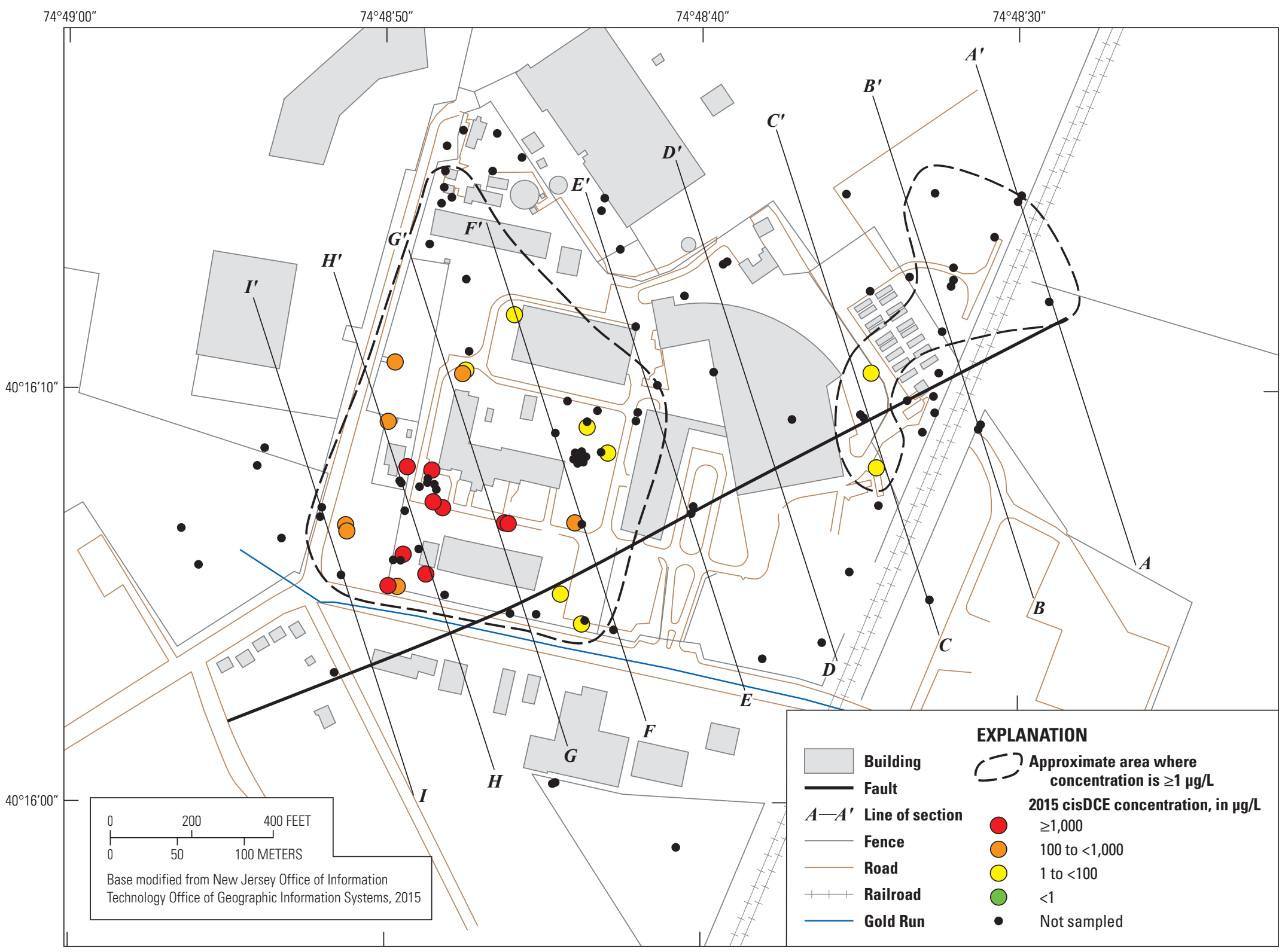

Figure 9. Areal extent of cis-1,2-dichloroethene contamination in wells at the former Naval Air Warfare Center, West Trenton, New Jersey, 2015. (cisDCE, cis-1,2-dichloroethene; $\mathrm{mg} / \mathrm{L}$, micrograms per liter) 


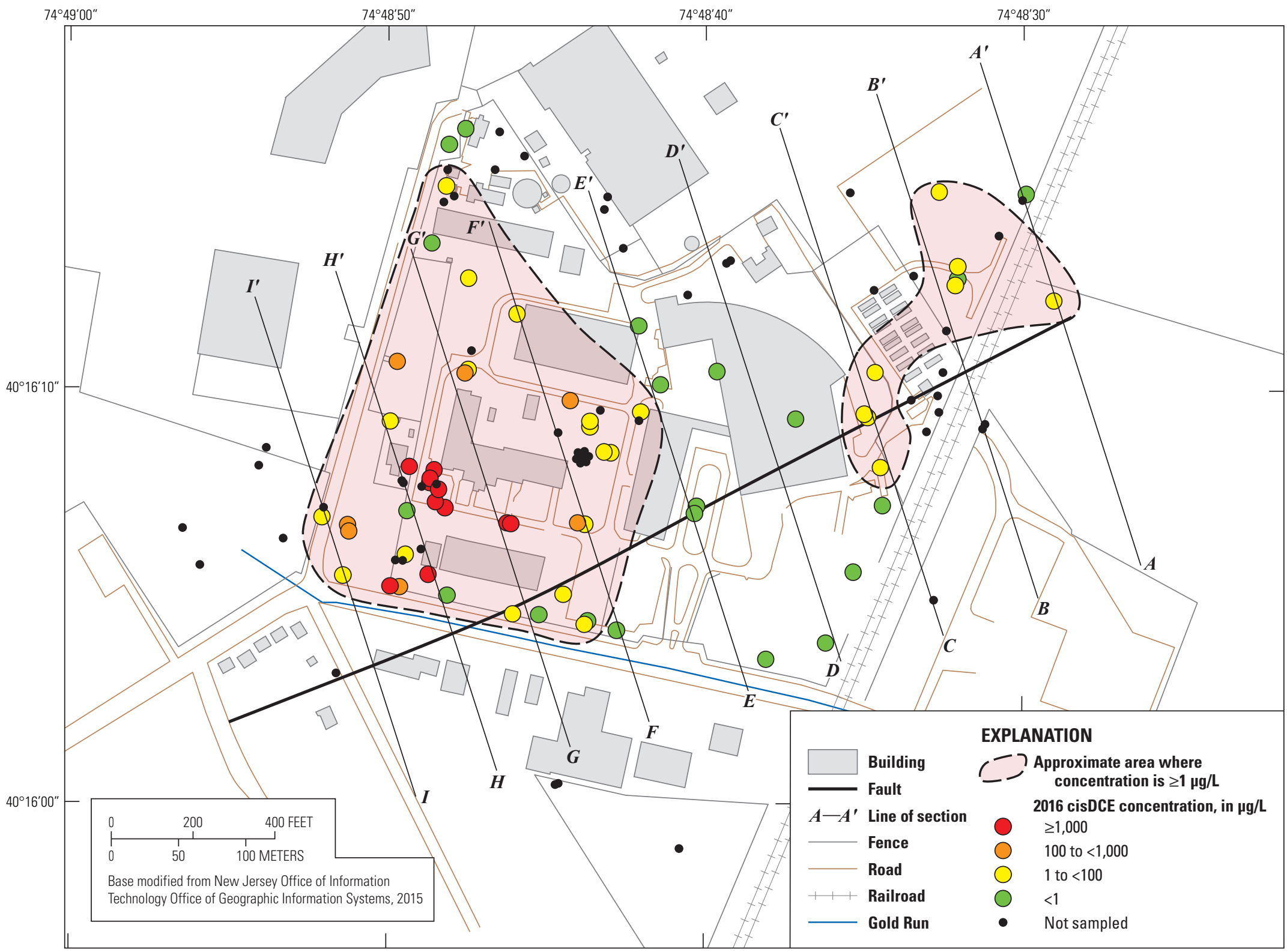

Figure 10. Areal extent of cis-1,2-dichloroethene contamination in wells at the former Naval Air Warfare Center, West Trenton, New Jersey, 2016. (cisDCE, cis-1,2-dichloroethene; mg/L, micrograms per liter) 


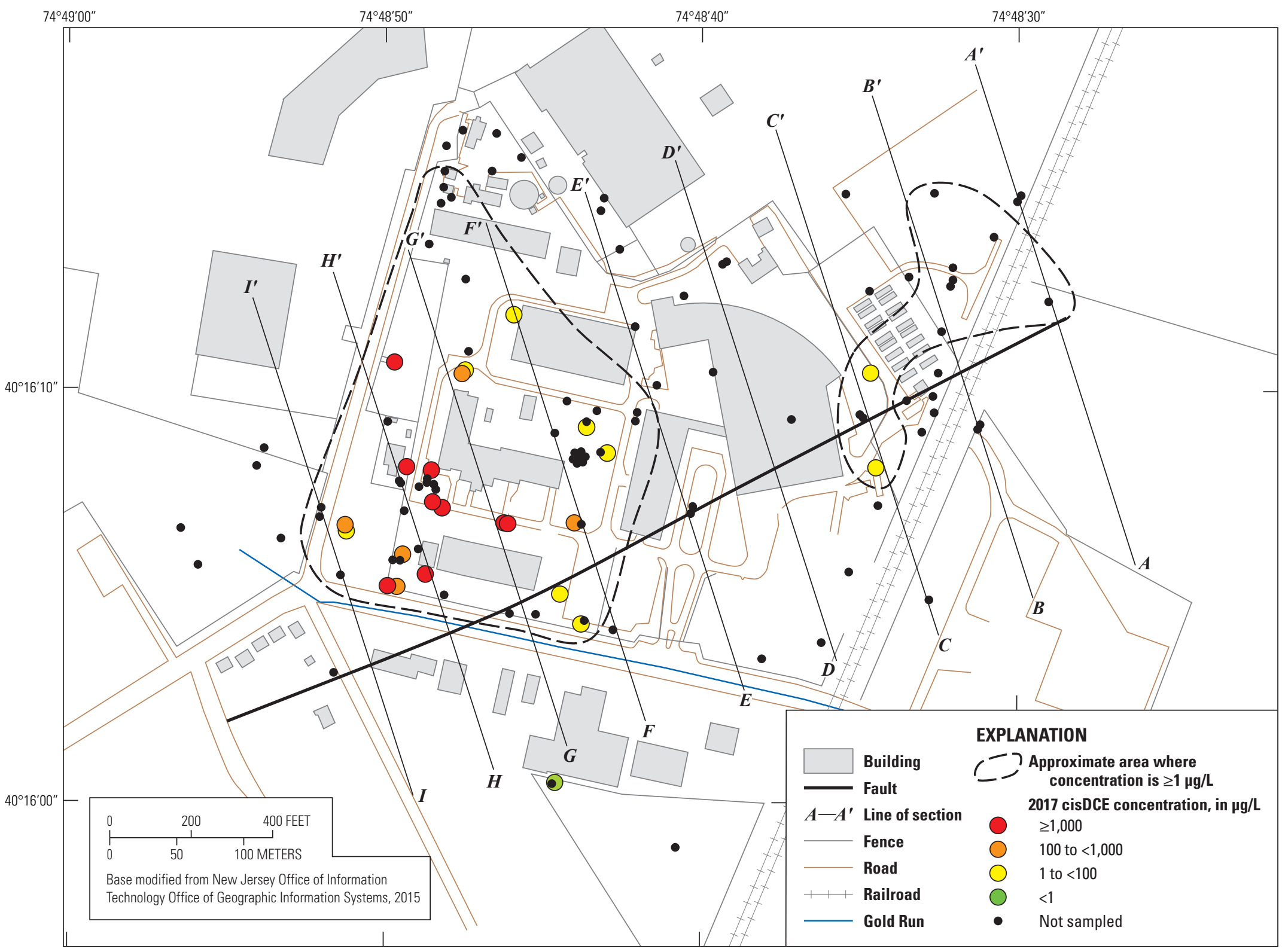

Figure 11. Areal extent of cis-1,2-dichloroethene contamination in wells at the former Naval Air Warfare Center, West Trenton, New Jersey, 2017. (cisDCE, cis-1,2-dichloroethene; $\mathrm{mg} / \mathrm{L}$, micrograms per liter) 


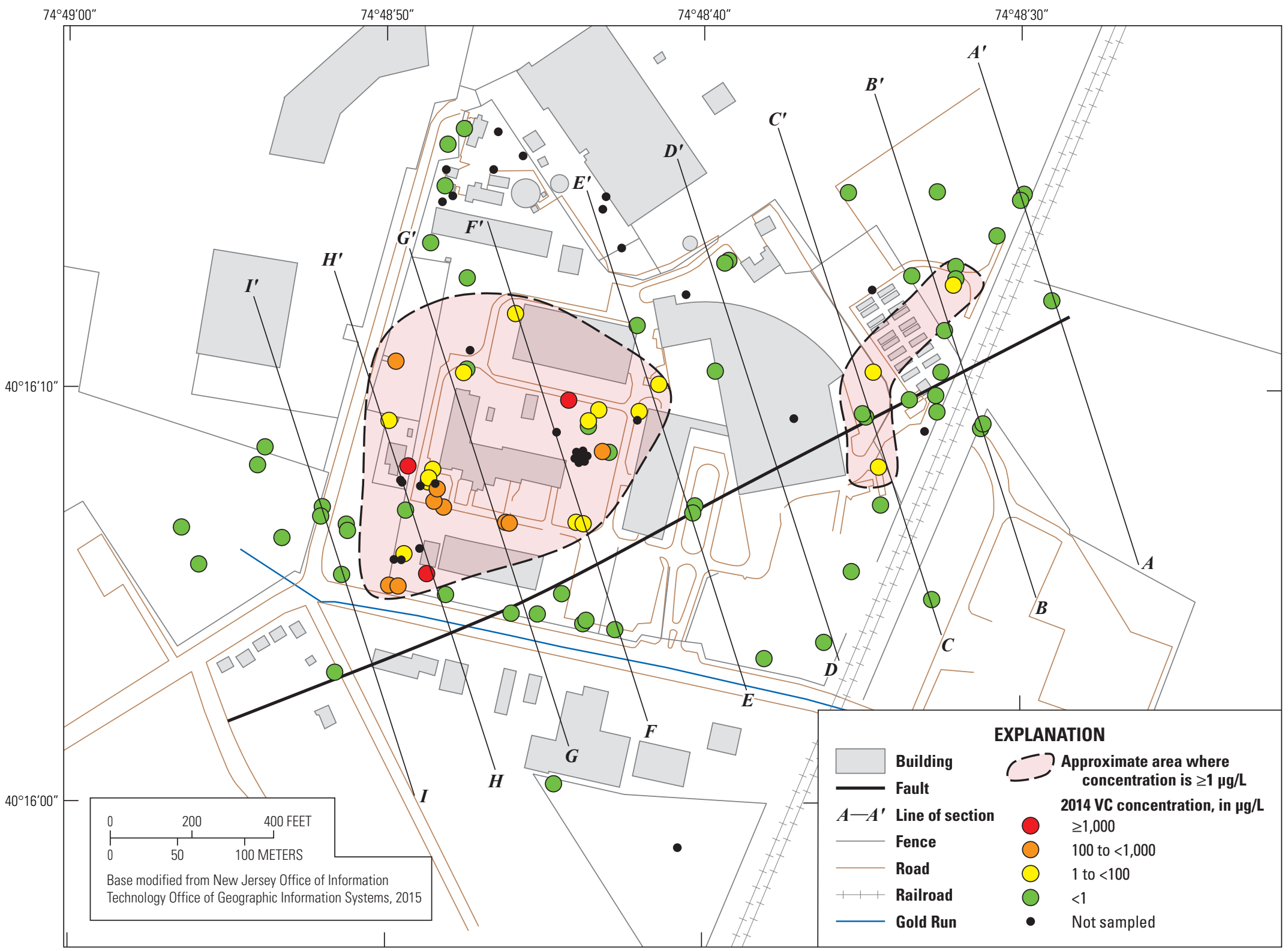

Figure 12. Areal extent of vinyl chloride contamination in wells at the former Naval Air Warfare Center, West Trenton, New Jersey, 2014. (VC, vinyl chloride; mg/L, micrograms per liter) 


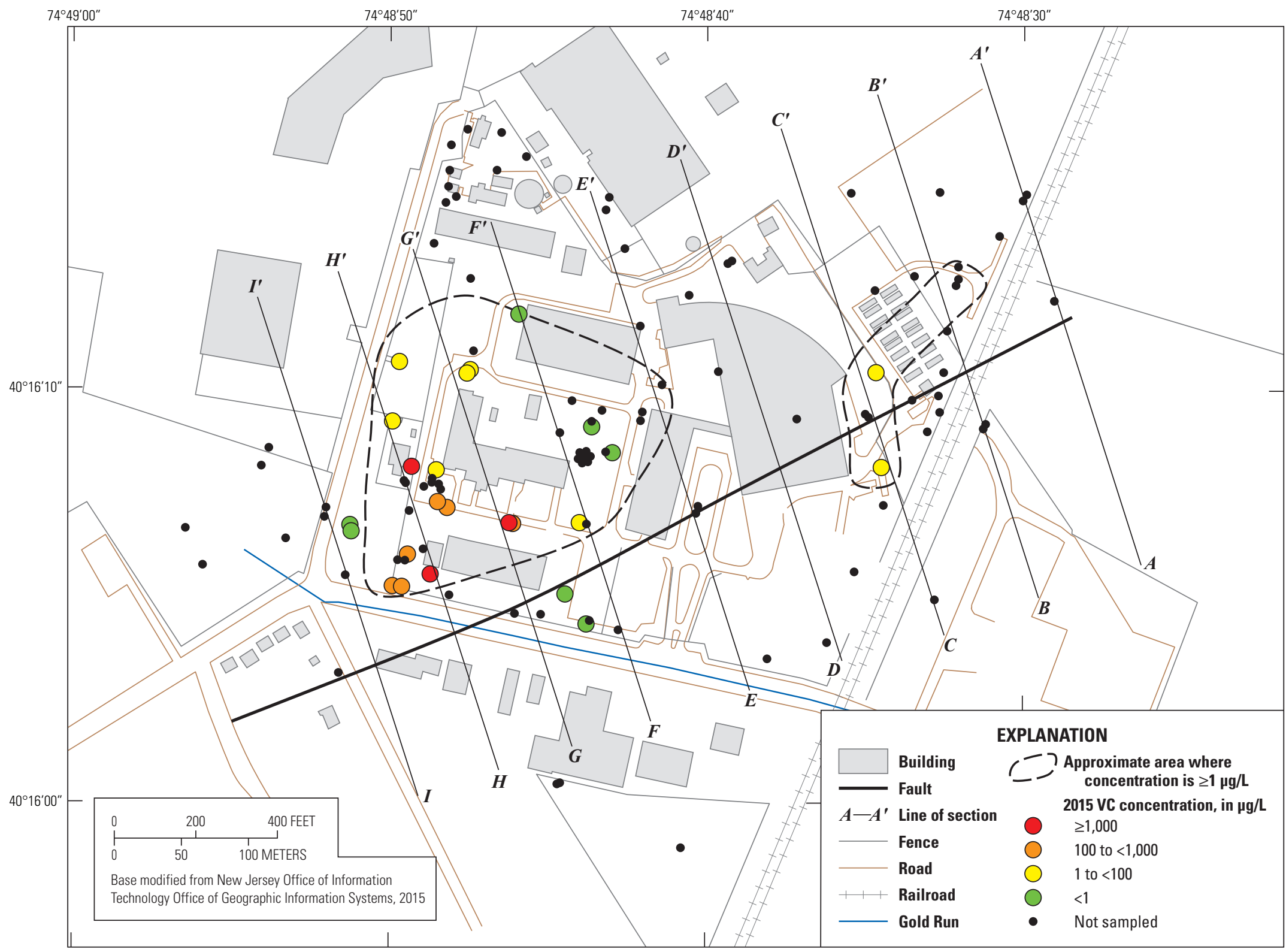

Figure 13. Areal extent of vinyl chloride contamination in wells at the former Naval Air Warfare Center, West Trenton, New Jersey, 2015. (VC, vinyl chloride; mg/L, micrograms per liter) 


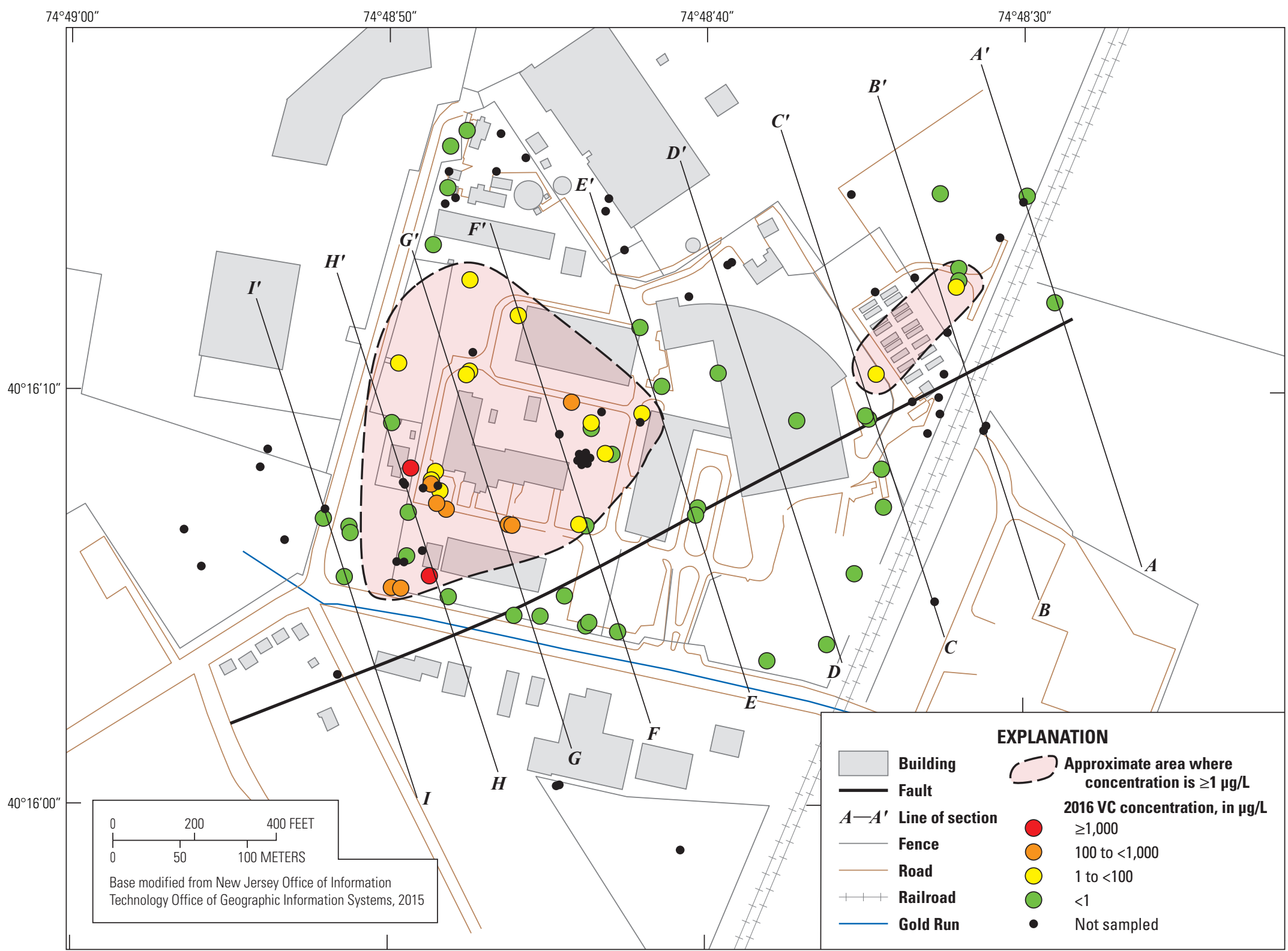

Figure 14. Areal extent of vinyl chloride contamination in wells at the former Naval Air Warfare Center, West Trenton, New Jersey, 2016. (VC, vinyl chloride; mg/L, micrograms per liter) 


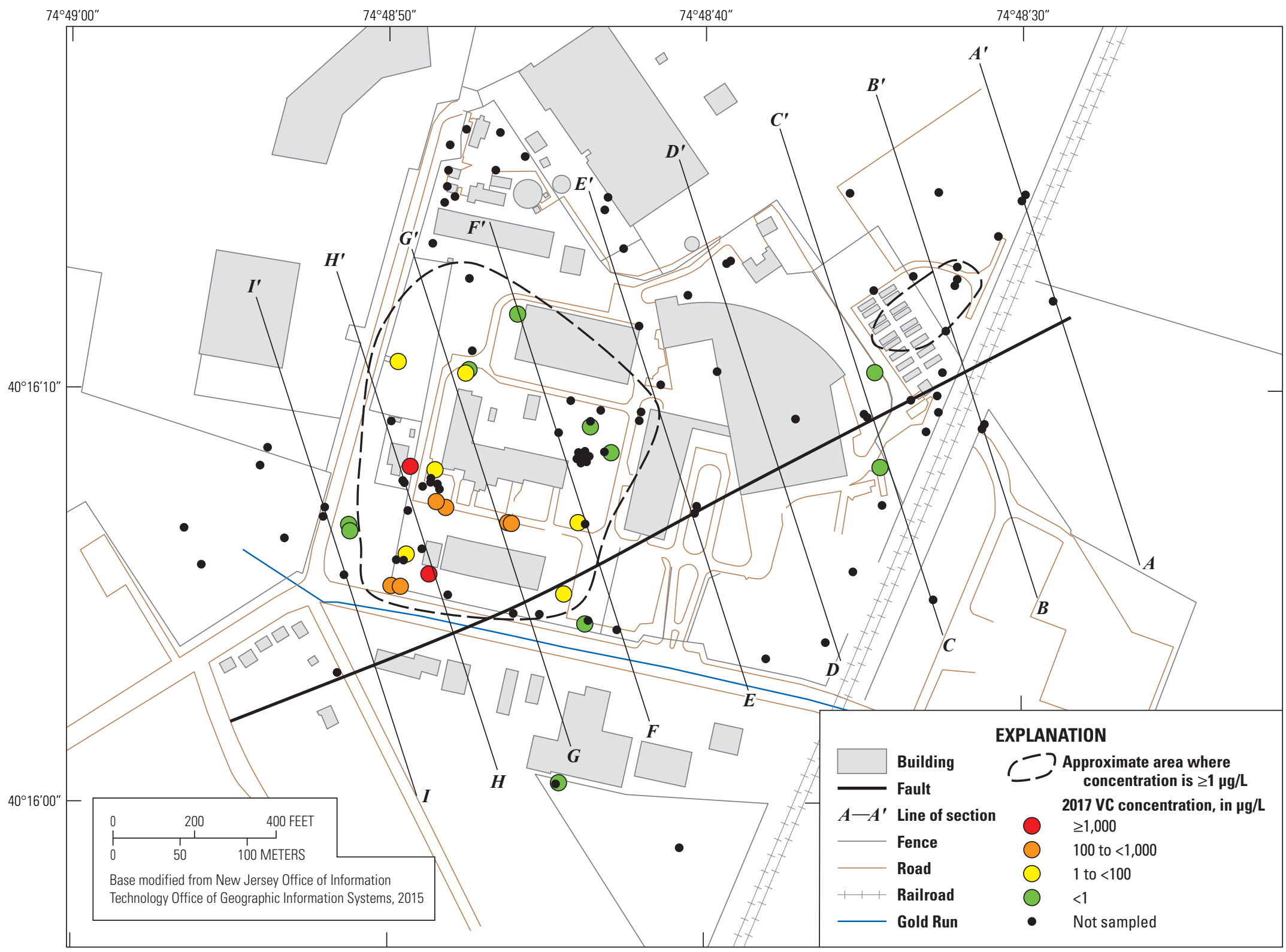

Figure 15. Areal extent of vinyl chloride contamination in wells at the former Naval Air Warfare Center, West Trenton, New Jersey, 2017. (VC, vinyl chloride; mg/L, micrograms per liter) 
2018). The VC concentrations were high in BRP02 because this well is in the area where jet fuel was stored and spilled, which provided a food source that stimulated degradation of TCE by the indigenous bacteria in the bedrock aquifer.

All five wells that showed substantial increases in VC concentration from 2014 to 2017 are in the Site 1 area. VC concentrations in wells 24BR, 70BR-10, 70BR-72, 73BR-A, and BRP01 increased by $\geq 26 \mu \mathrm{g} / \mathrm{L}$. Wells 70BR-10, 70BR-72, 73BR-A, and BRP01 are in the areas of the 2005 and 2008 bioaugmentation studies, so their increased VC concentrations may be due to the more favorable geochemical conditions created by these studies in the bedrock aquifer.

The Site 3 areas of VC contamination were smaller than the Site 1 areas of VC contamination at NAWC between 2014 and 2017. The VC concentrations in wells in Site 3 were generally one order of magnitude lower than most of the VC concentrations in wells in Site 1. In 2014 (fig. 12), the area of $\mathrm{VC}$ contamination appeared to originate near the former wastewater lagoon and sludge drying beds (fig. 1), similar to TCE and cisDCE. In 2016, the Site 3 VC contamination area was slightly smaller because concentrations in well 54BR decreased from slightly $>1 \mu \mathrm{g} / \mathrm{L}$ to slightly $<1 \mu \mathrm{g} / \mathrm{L}$ (fig. 14). The likely areal extents of VC contamination in 2015 (fig. 13) and 2017 (fig. 15) in the Site 3 area were estimated based on the smaller number of wells sampled during these two years and data from the years before and after. The highest VC concentrations in wells in the Site 3 area were all $<2.5 \mu \mathrm{g} / \mathrm{L}$. There was no evidence of any VC contamination in any offsite wells to the east of the railroad tracks during 2014-17.

\section{Vertical Extent of VOC Contamination at NAWC During 2014-17}

To show the variation in VOC concentrations with depth at NAWC, wells were projected onto a set of 13 lines of section labeled $\mathrm{A}-\mathrm{A}^{\prime}$ through $\mathrm{K}-\mathrm{K}^{\prime}$ across the site from east to west (shown on figures 4-15). Concentrations of VOCs are plotted on these lines of section and shown in figures 16-42. Sections A-A' through D-D' include the wells in the eastern Site 3 area of NAWC. Sections E-E' through I-I' include the wells in the western Site 1 area of NAWC. Sections J-J' and $\mathrm{K}-\mathrm{K}^{\prime}$ include wells west of the NAWC site on the airport property. Because all wells on these the two sections had VOC concentrations $<0.16 \mu \mathrm{g} / \mathrm{L}$ in all samples collected during 2014-17, these sections are not plotted or discussed. Wells with TCE, cisDCE, and VC concentrations $\geq 1 \mu \mathrm{g} / \mathrm{L}$ are defined as contaminated and are included on cross sections within dashed vertical areas with pink shading. VOC results from wells sampled in 2014 were compared to the most recent VOC results from wells sampled in 2015-2017 to see if any substantial concentration changes had occurred. All wells projected to the cross sections are shown at their correct elevations. However, because these are projections, the tops of some wells may appear to be below land surface.

\section{Vertical Extent of TCE Contamination During 2014-17}

\section{Site 3 Eastern Sections A-A' to D-D'}

TCE concentrations in wells along cross section A-A' in 2014 are shown in figure 16A, and the most recent TCE concentration in wells along section A-A' from 2015 to 2017 are shown in figure 16B. In 2014, low TCE concentrations $(1.5 \mu \mathrm{g} / \mathrm{L}$ and $3.7 \mu \mathrm{g} / \mathrm{L})$ were measured in samples from wells $11 \mathrm{BR}$ and 50BR that defined a vertical area of TCE contamination that extended from the shallow groundwater to about 80 $\mathrm{ft}$ below land surface. The main difference between 2014 and 2015-17 is that the TCE concentration in well 11BR decreased from $1.5 \mu \mathrm{g} / \mathrm{L}$ in 2014 to $<0.6 \mu \mathrm{g} / \mathrm{L}$ in 2016 . This slight decrease in concentration substantially reduced the area of vertical contamination shown on this cross section (fig. 16B).

Similarly, for section B-B', the TCE concentrations in 2014 and the most recent TCE concentrations from 2015 to 2017 are shown in figures $17 \mathrm{~A}$ and 17B. In 2014, TCE contamination of $\geq 1 \mu \mathrm{g} / \mathrm{L}$ was present from the shallow groundwater to about $96 \mathrm{ft}$ below land surface in well 51BR (fig. 17A). The main difference between the cross sections is that TCE concentrations in well 12BR decreased from $1.0 \mu \mathrm{g} / \mathrm{L}$ in 2014 to $<0.53 \mu \mathrm{g} / \mathrm{L}$ in 2016 and in well $51 \mathrm{BR}$ decreased from $1.8 \mu \mathrm{g} / \mathrm{L}$ in 2014 to $<1.5 \mu \mathrm{g} / \mathrm{L}$ in 2016 (fig. 17B). These slight decreases in the TCE concentrations dramatically reduced the vertical area of contamination shown along this section of the aquifer between the two periods.

Along section $\mathrm{C}-\mathrm{C}^{\prime}$, the TCE concentrations in 2014 were $<0.13 \mu \mathrm{g} / \mathrm{L}$ in 2 of the 3 shallow wells (11MW1 and 30S) with the third shallow well (31S) having only $1.1 \mu \mathrm{g} / \mathrm{L}$ (fig. 18A). Extraction well 48BR was the most contaminated well along this section in 2014 with a maximum TCE concentration of $520 \mu \mathrm{g} / \mathrm{L}$. Wells $31 \mathrm{BR}$ and 54BR had TCE concentrations of $6.9 \mu \mathrm{g} / \mathrm{L}$ and $51 \mu \mathrm{g} / \mathrm{L}$, respectively, in 2014 . TCE concentrations remained approximately the same during 2015-17 in all these wells (fig. 18B). TCE in 48BR varied over the study period but ended in 2017 at a concentration $(570 \mu \mathrm{g} / \mathrm{L})$ similar to that in 2014. The most recent TCE concentrations in $31 \mathrm{BR}$ and 54BR were $7.8 \mu \mathrm{g} / \mathrm{L}$ and $29 \mu \mathrm{g} / \mathrm{L}$, respectively, which were similar to their 2014 values.

The TCE concentrations in extraction well 48BR presumably originated from TCE contamination being drawn down from the Site 3 former wastewater lagoon and sludge disposal areas. The fact that well 54BR had TCE concentrations of 51 and $29 \mu \mathrm{g} / \mathrm{L}$ from 2014 to 2017 indicates that TCE contamination had moved vertically downward to a depth of $200 \mathrm{ft}$ below land surface and that TCE contamination was present on the southern side of the fault along this section.

All TCE concentrations in wells along section D-D' in 2014 were $<1 \mu \mathrm{g} / \mathrm{L}$ (fig. 19A). From 2015 to 2017, the most recent TCE concentrations in all wells sampled along this section remained at $<1 \mu \mathrm{g} / \mathrm{L}$ (fig. 19B). 


\section{A. 2014 TCE concentrations}

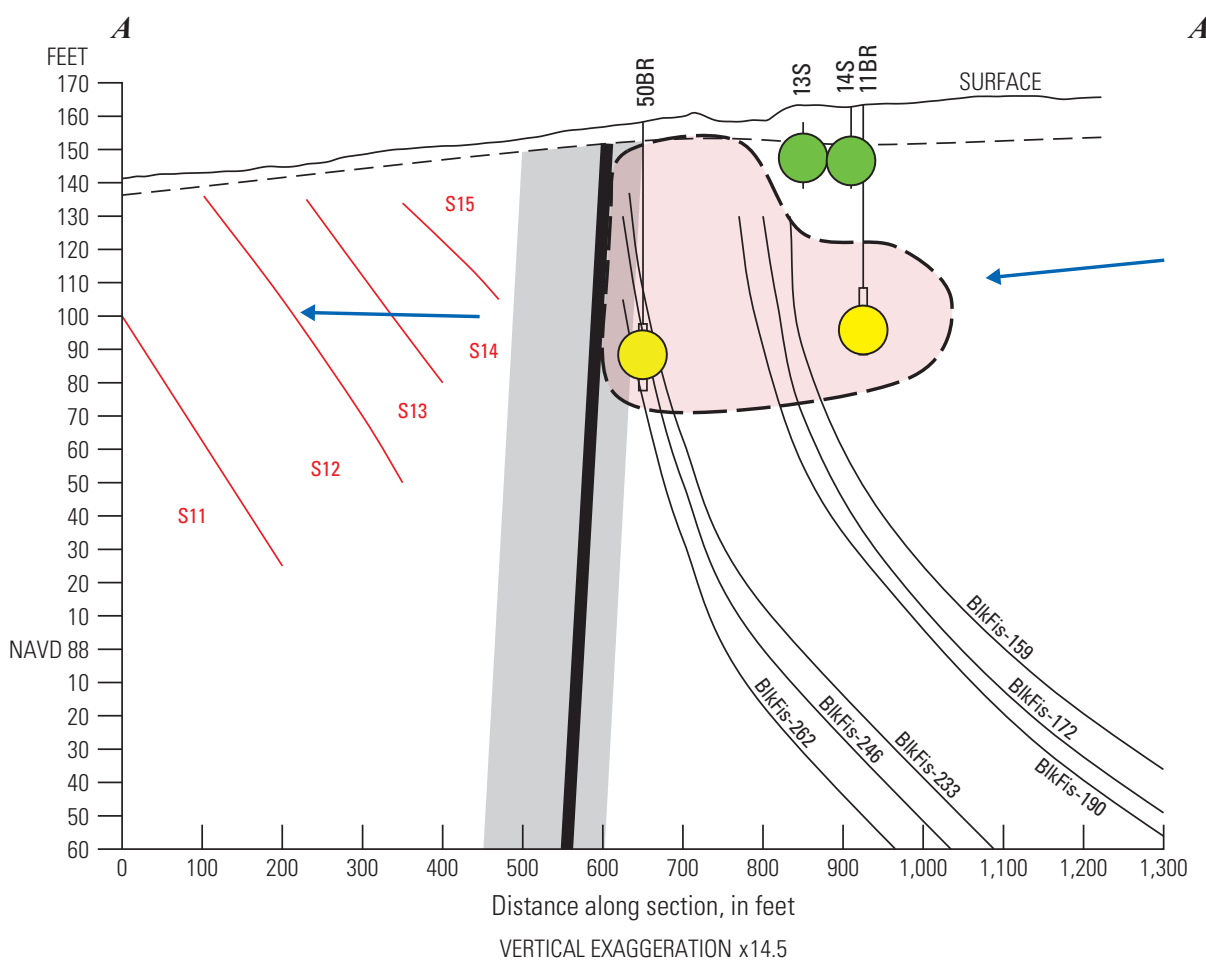

\section{B. 2015-17 TCE most recent concentrations}

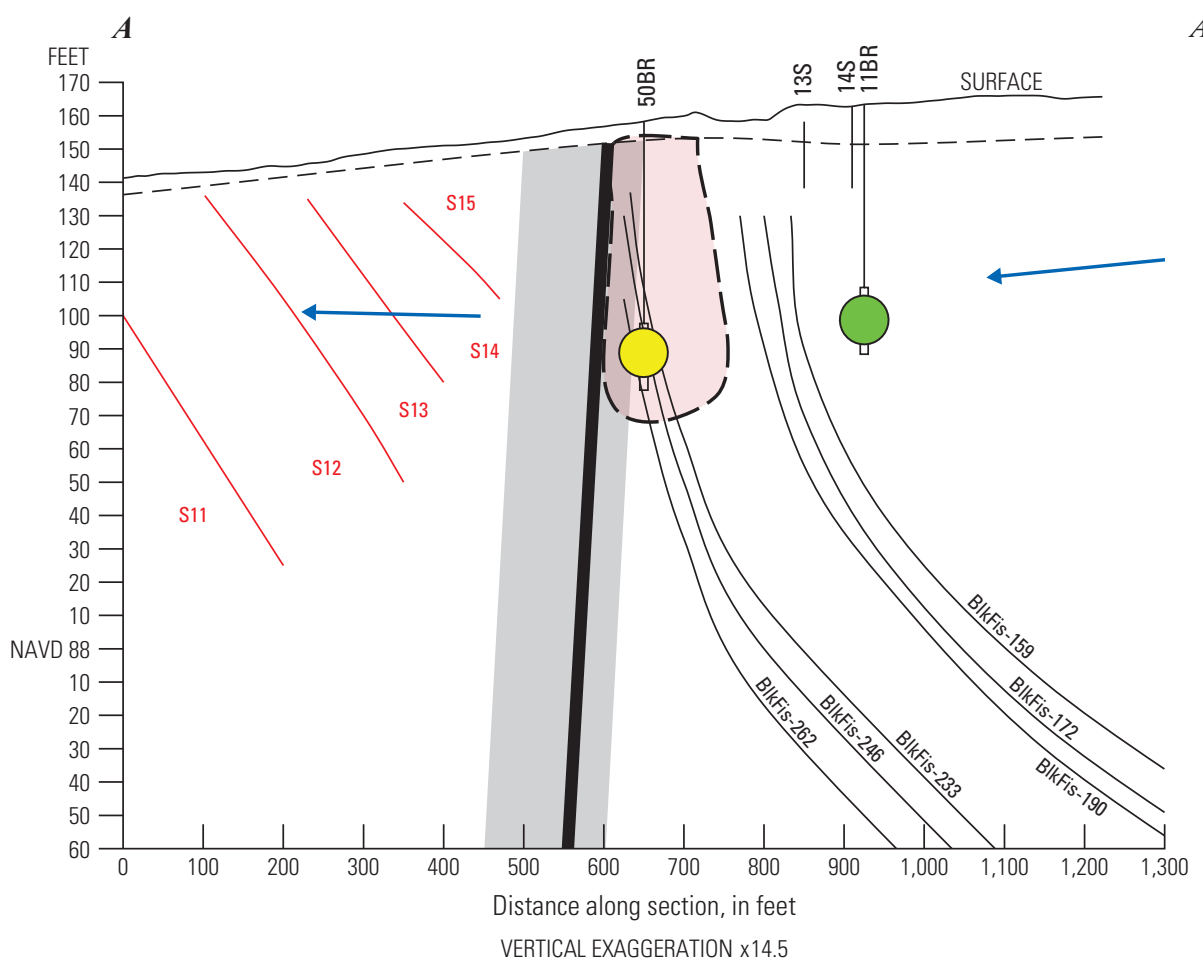

\begin{tabular}{|c|c|}
\hline & EXPLANATION \\
\hline & Approximate area where \\
\hline & Fault zone \\
\hline & Fault \\
\hline BlkFis-172 & $\begin{array}{l}\text { Lockatong Formation fissile } \\
\text { unit and identifier }\end{array}$ \\
\hline S12 & $\begin{array}{l}\text { Stockton Formation unit top } \\
\text { and identifier }\end{array}$ \\
\hline & Bottom of saprolite \\
\hline & Flow direction \\
\hline & $\begin{array}{l}\text { Surficial or bedrock well } \\
\text { and indentifier }\end{array}$ \\
\hline & $\begin{array}{l}\text { TCE concentration, in } \mu \mathrm{g} / \mathrm{L} \\
\geq 1,000\end{array}$ \\
\hline & 100 to $<1,000$ \\
\hline & 1 to $<100$ \\
\hline & $<1$ \\
\hline
\end{tabular}

Figure 16. Vertical extent of trichloroethene concentrations in wells along section $\mathrm{A}-\mathrm{A}^{\prime}$ in $A, 2014$ and $B, 2015-17$, former Naval Air Warfare Center, West Trenton, New Jersey. (TCE, trichloroethene; mg/L, micrograms per liter) 


\section{A. 2014 TCE concentrations}

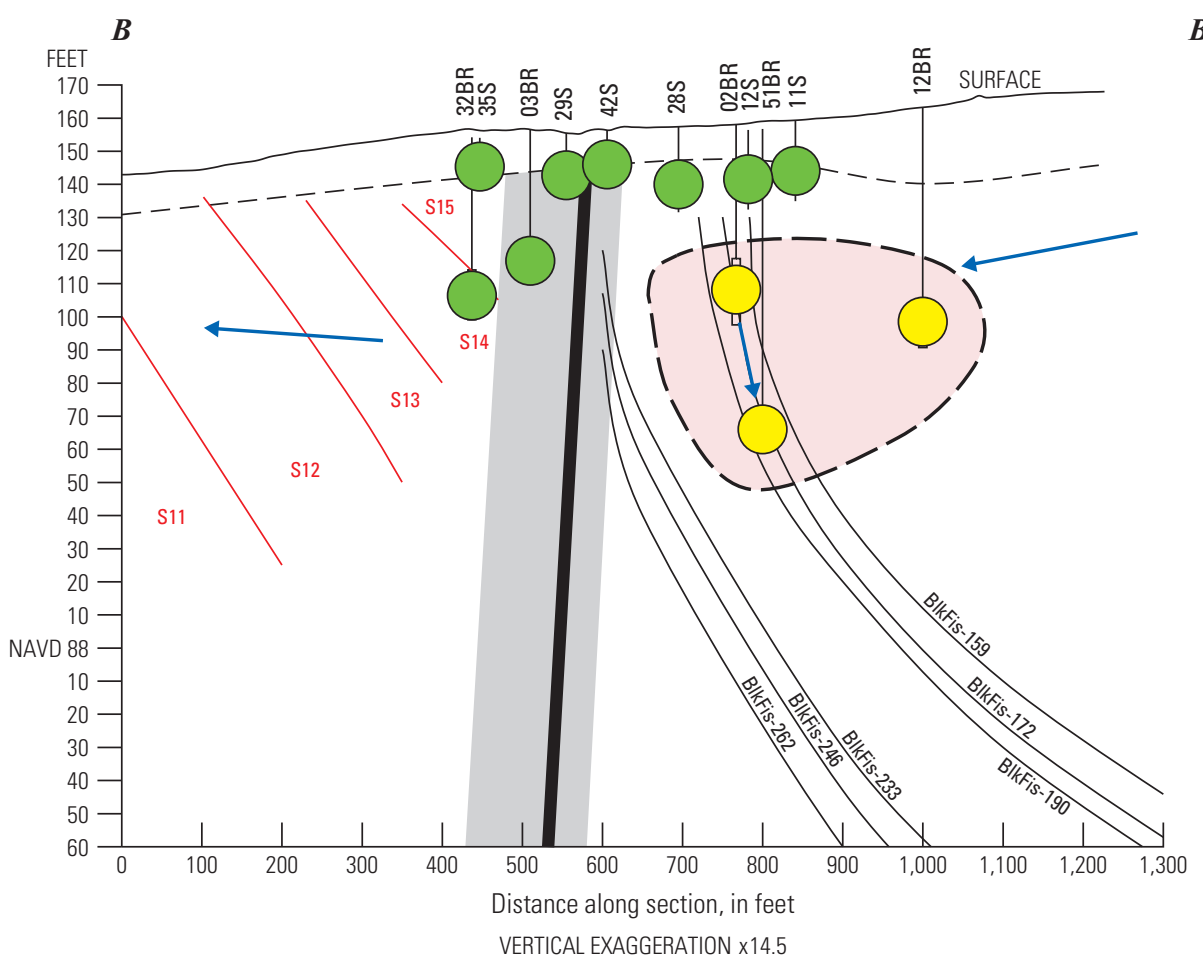

\section{B. 2015-17 TCE most recent concentrations}

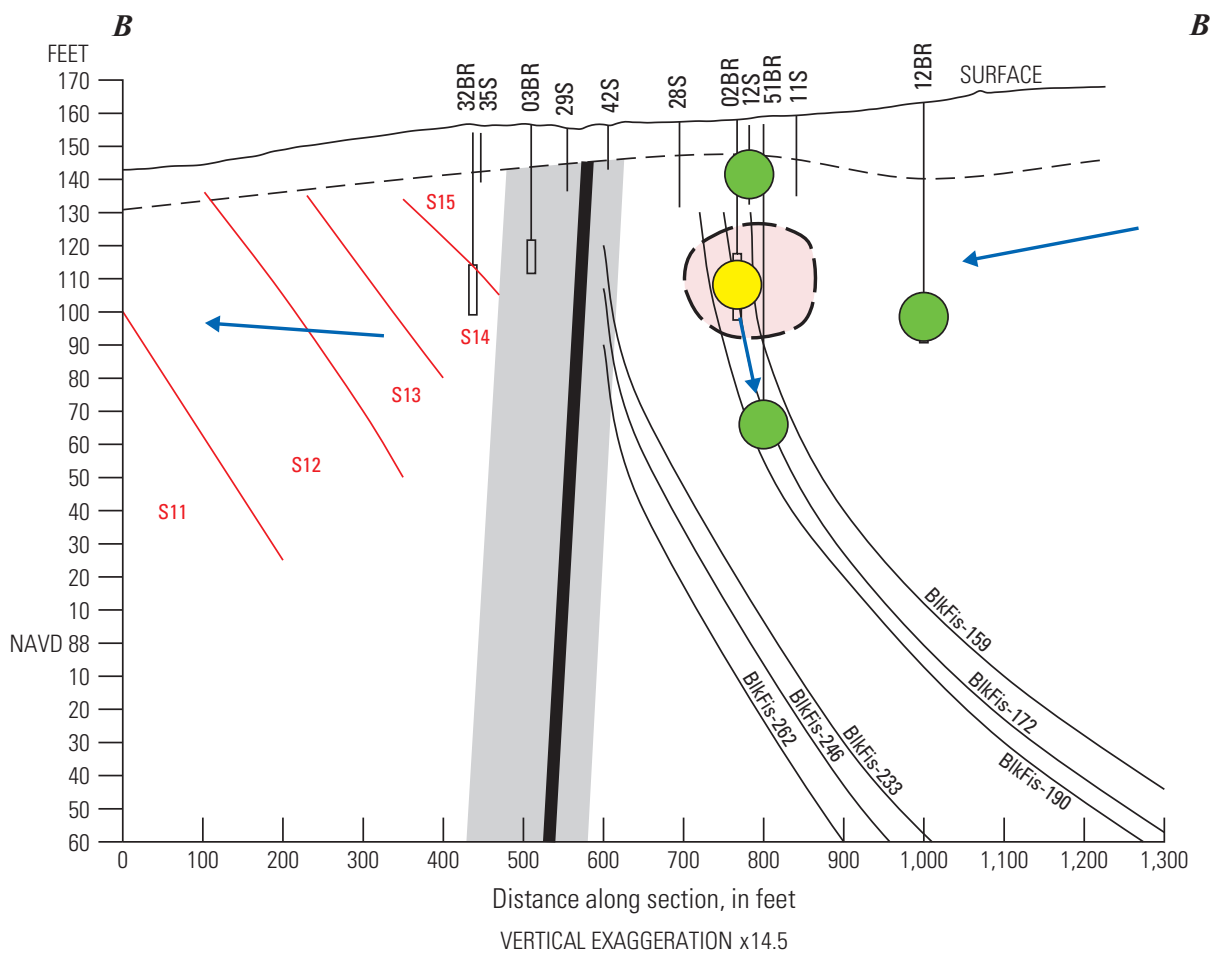

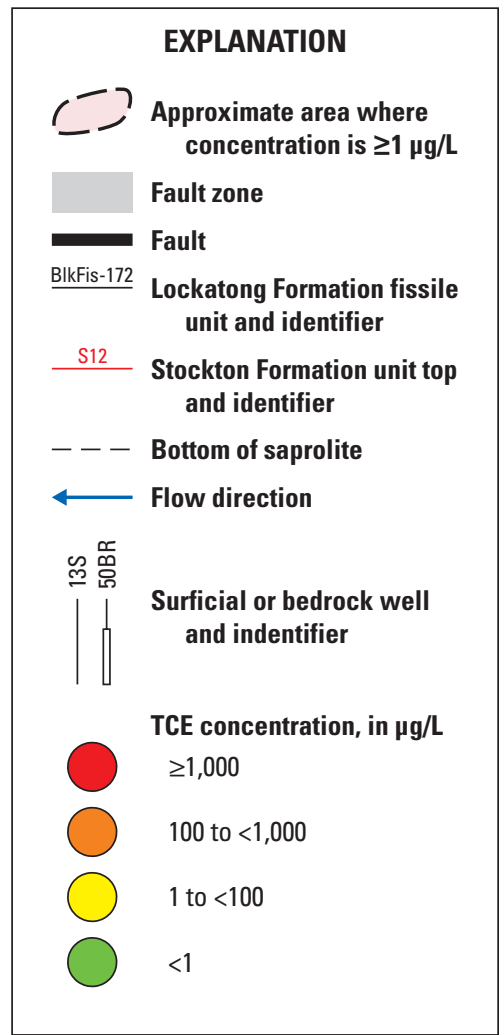

Figure 17. Vertical extent of trichloroethene concentrations in wells along section $B-B$ ' in $A, 2014$ and $B, 2015-17$, former Naval Air Warfare Center, West Trenton, New Jersey. (TCE, trichloroethene; $\mathrm{mg} / \mathrm{L}$, micrograms per liter) 


\section{A. 2014 TCE concentrations}

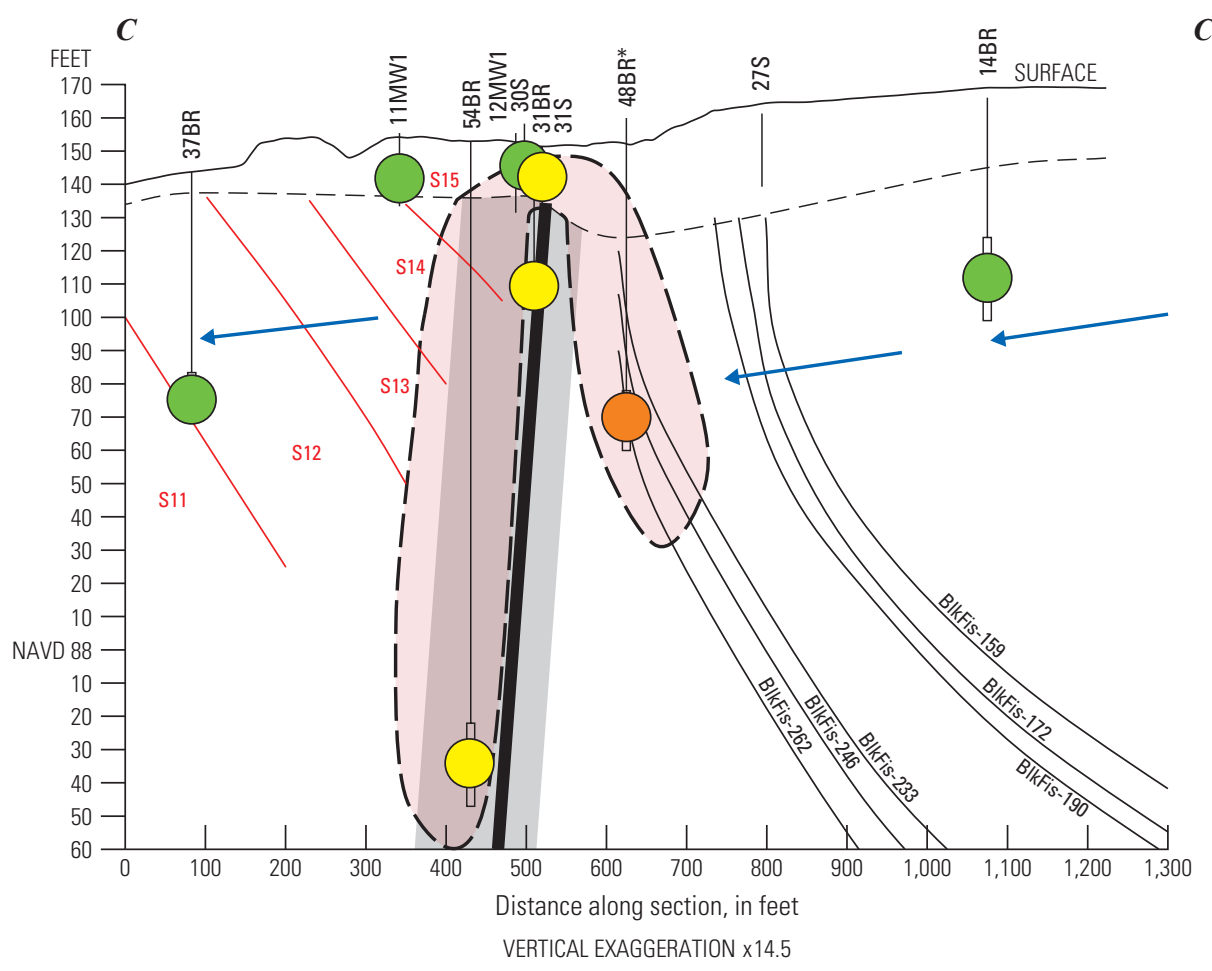

\section{B. 2015-17 TCE most recent concentrations}

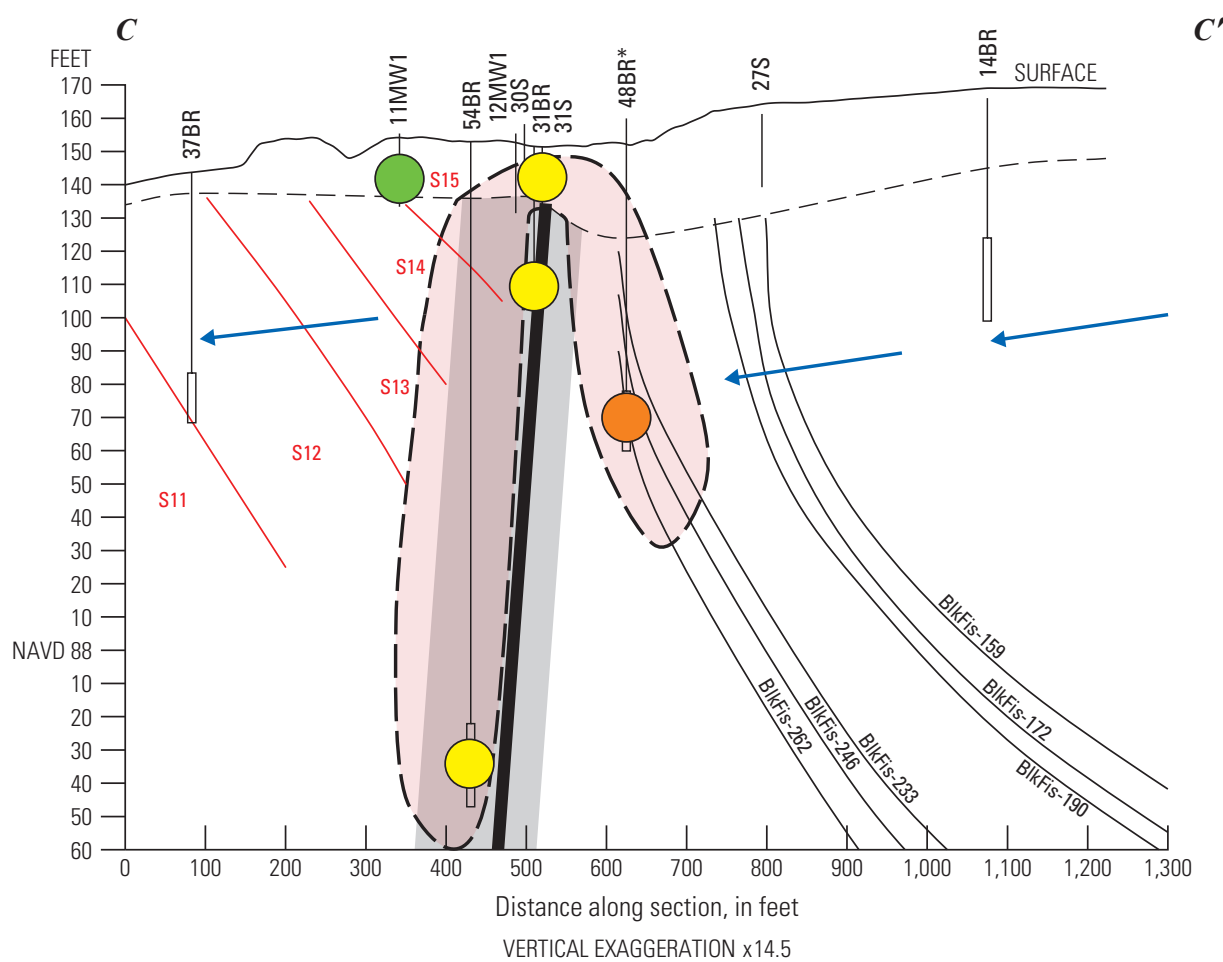

EXPLANATION
Fault zone
concentration is $\geq 1 \mu \mathrm{g} / \mathrm{L}$
Fault

Figure 18. Vertical extent of trichloroethene concentrations in wells along section $C-C^{\prime}$ in $A, 2014$ and $B, 2015-17$, former Naval Air Warfare Center, West Trenton, New Jersey. (TCE, trichloroethene; mg/L, micrograms per liter) 


\section{A. 2014 TCE concentrations}

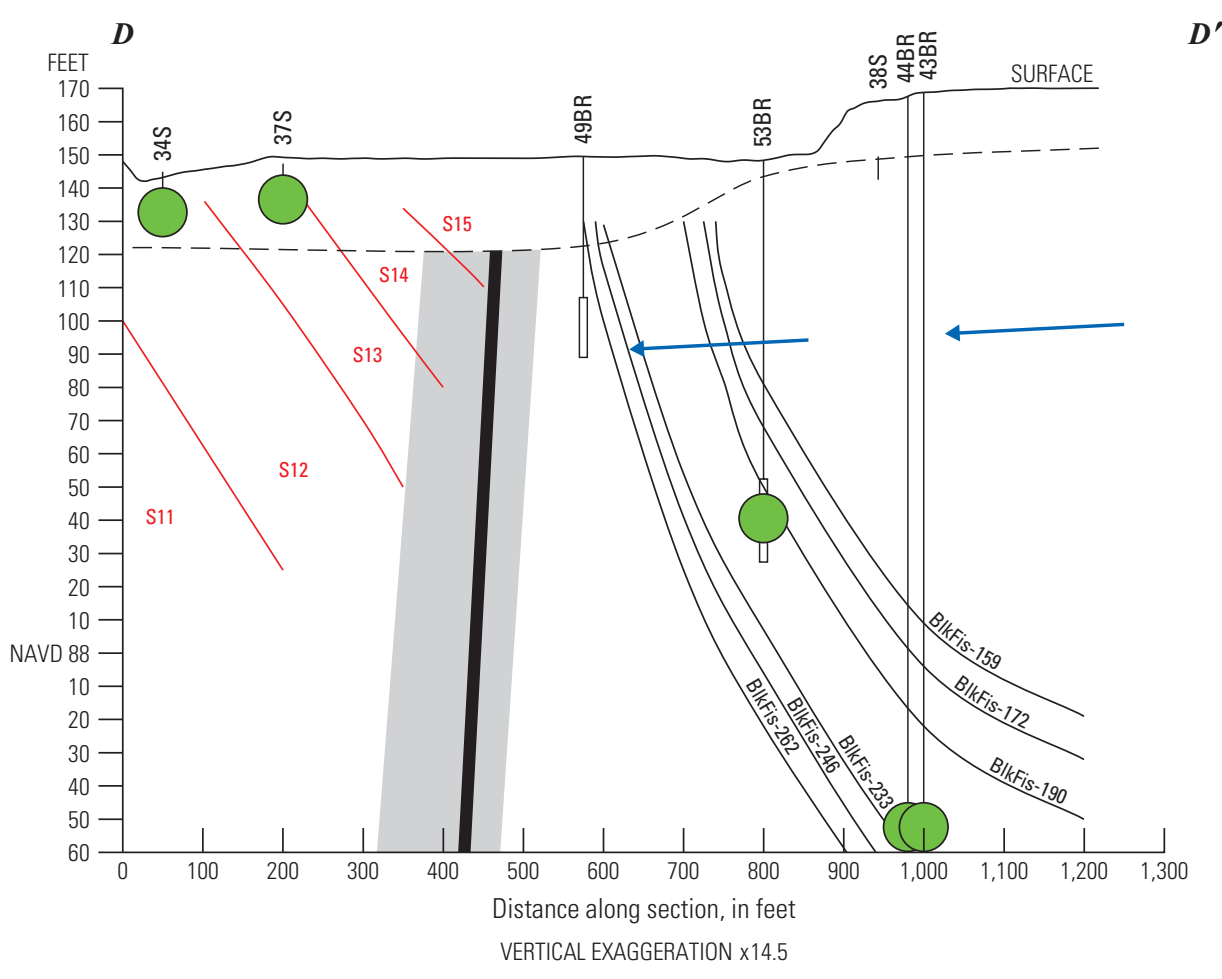

\section{B. 2015-17 TCE most recent concentrations}

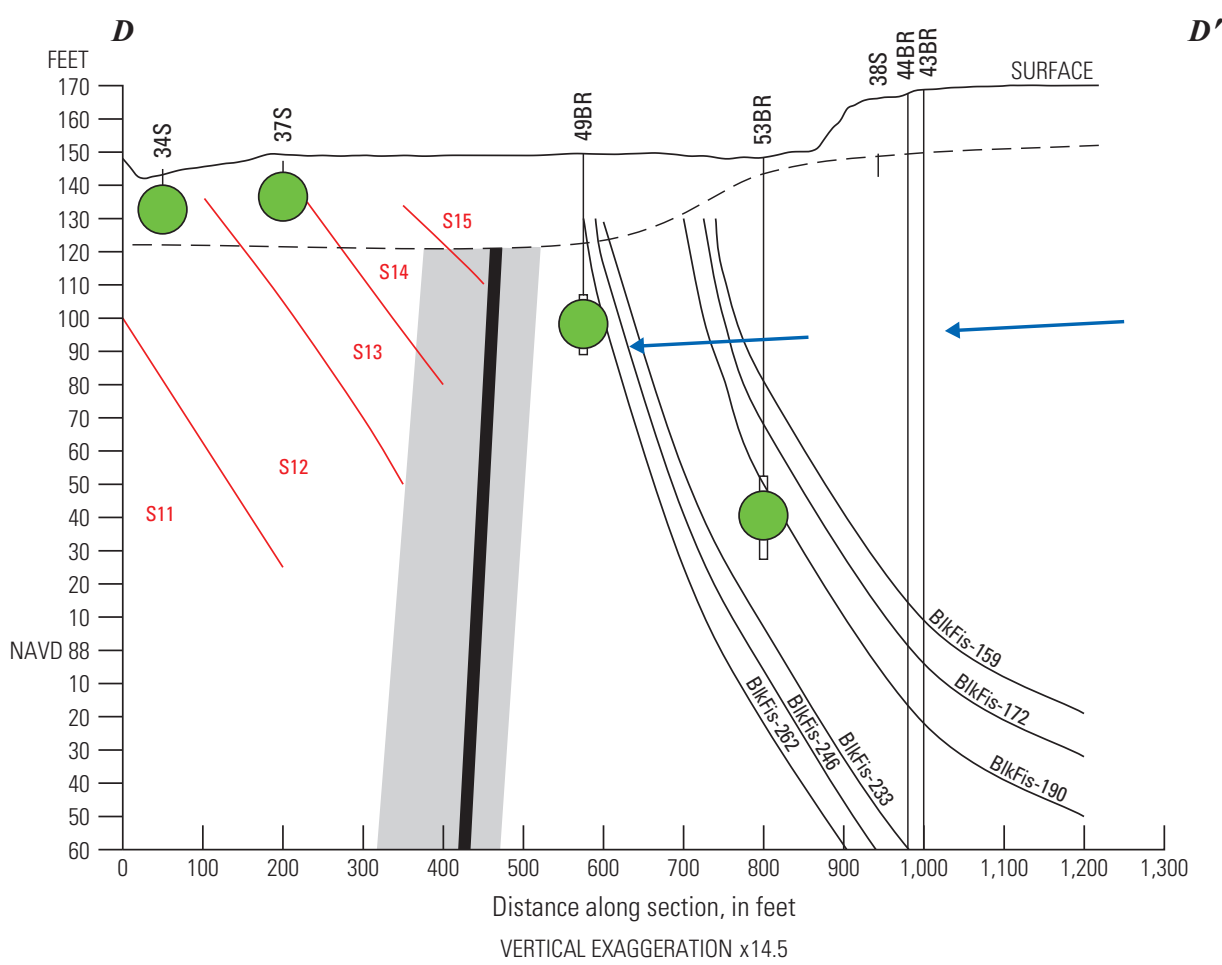

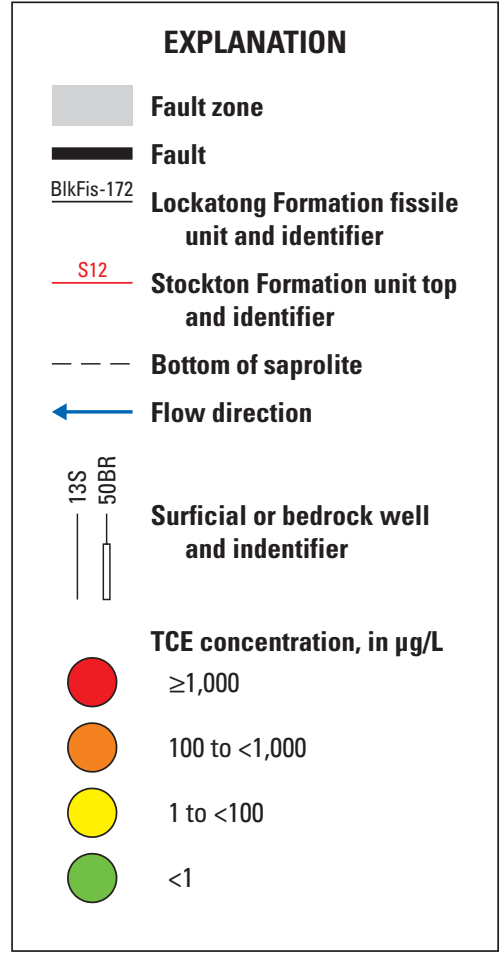

Figure 19. Vertical extent of trichloroethene concentrations in wells along section D-D' in A, 2014 and B, 2015-17, former Naval Air Warfare Center, West Trenton, New Jersey. (TCE, trichloroethene; mg/L, micrograms per liter) 


\section{Site 1 Western Sections E-E' to I-I'}

Farther to the west, TCE concentrations in wells along section E-E' in 2014 are shown in figure 20A, and concentrations from 2015 to 2017 are shown in figure 20B. Only two wells along this section had TCE concentrations $\geq 1 \mu \mathrm{g} / \mathrm{L}, 16 \mathrm{~S}$ with $1.1 \mu \mathrm{g} / \mathrm{L}$ and $41 \mathrm{BR}$ with $2.0 \mu \mathrm{g} / \mathrm{L}$ in 2014 . However, the most recent 2015-17 samples from these wells had concentrations $<1.2 \mu \mathrm{g} / \mathrm{L}$. The slight decrease in TCE concentrations in these two wells eliminated the shaded TCE-contaminated area along this section in figure $20 \mathrm{~B}$.

The vertical extent of TCE contamination in wells along section $\mathrm{F}-\mathrm{F}^{\prime}$ is shown for 2014 in figure 21A and for 2015-17 in figure $21 \mathrm{~B}$. The most contaminated well on this section for both time periods is deep well 46BR with TCE concentrations of $2,000 \mu \mathrm{g} / \mathrm{L}$ in 2014 to $2,100 \mu \mathrm{g} / \mathrm{L}$ in 2017 . The TCE in this well was apparently drawn downdip along bedding partings from the TCE-contaminated Site 1 area between Buildings 40 and 41 to $221 \mathrm{ft}$ below land surface by the pump sampling methods used in this well and by the pumping of nearby extraction well 45BR. Other contaminated wells along section F-F' in 2014 included wells 04BR $(490 \mu \mathrm{g} / \mathrm{L})$ and 23BR $(120 \mu \mathrm{g} / \mathrm{L})$. TCE concentrations in both wells decreased during 2015-17, with 04BR decreasing to $310 \mu \mathrm{g} / \mathrm{L}$ and $23 \mathrm{BR}$ decreasing to $58 \mu \mathrm{g} / \mathrm{L}$. TCE in well 38BR decreased from 2.7 $\mu \mathrm{g} / \mathrm{L}$ in 2014 to $<1 \mu \mathrm{g} / \mathrm{L}$ in 2016 , which resulted in the split in the shaded TCE-contaminated area shown on figure 21B.

TCE contamination was vertically widespread along section $\mathrm{G}-\mathrm{G}^{\prime}$ from shallow groundwater to a depth of at least 100 $\mathrm{ft}$ below land surface. The ranges in concentrations in wells along section $\mathrm{G}-\mathrm{G}^{\prime}$ are shown on figures $22 \mathrm{~A}$ and $22 \mathrm{~B}$. Wells 07BR, 24BR, 29BR, and 08BR were the most contaminated in 2014 and remained so during 2015-17. Well 24BR had the highest TCE concentrations ranging from $20,000 \mu \mathrm{g} / \mathrm{L}$ in 2014 to $29,000 \mu \mathrm{g} / \mathrm{L}$ in 2017 . The TCE concentration in well 29BR doubled from 2014 to $2017(1,900 \mu \mathrm{g} / \mathrm{L}$ to $3,800 \mu \mathrm{g} / \mathrm{L})$, indicating that this extraction well had started drawing in water with higher TCE concentrations over the last few years.

Section $\mathrm{H}-\mathrm{H}^{\prime}$ accounted for most of the Site 1 wells with TCE concentrations $\geq 1,000 \mu \mathrm{g} / \mathrm{L}$ during 2014-17 (fig. 23). Six of the extraction wells that pump water to the pump-andtreat plant (fig. 1) are located along this section. Extraction well 56BR (165 ft deep) had the highest TCE concentrations, ranging from $12,000 \mu \mathrm{g} / \mathrm{L}$ in 2014 to $10,000 \mu \mathrm{g} / \mathrm{L}$ in 2017 . Extraction well 45BR (210 ft deep) had TCE concentrations ranging from $2,000 \mu \mathrm{g} / \mathrm{L}$ in 2014 to $2,100 \mu \mathrm{g} / \mathrm{L}$ in 2017 . These two wells were the deepest wells found to be contaminated along this section. These two wells were open to two watertransmitting layers that underlie the area between Buildings 40 and 41 where most of the Site 1 TCE spills and leaks occurred in the past. Therefore, multiple layers deep in the fractured rock aquifer were highly contaminated with TCE by the past activities at NAWC.

Four wells had TCE concentrations $\geq 1,000 \mu \mathrm{g} / \mathrm{L}$ in 2014 (fig. 23A) and 2015-17 (fig. 23B). Well 25BR had TCE concentrations that ranged from $7,000 \mu \mathrm{g} / \mathrm{L}$ in 2014 to $6,100 \mu \mathrm{g} / \mathrm{L}$ in 2017. Extraction well 15BR had TCE concentrations that ranged from $3,900 \mu \mathrm{g} / \mathrm{L}$ in 2014 to $3,300 \mu \mathrm{g} / \mathrm{L}$ in 2017 . Well 71BR-A had TCE concentrations that ranged from $2,800 \mu \mathrm{g} / \mathrm{L}$ in 2014 to $4,300 \mu \mathrm{g} / \mathrm{L}$ in 2017. Extraction well BRP02 had TCE concentrations that ranged from $1,300 \mu \mathrm{g} / \mathrm{L}$ in 2014 to $2,500 \mu \mathrm{g} / \mathrm{L}$ in 2017 . These results show that, although concentrations fluctuated in these wells, all remained highly contaminated with TCE from 2014 to 2017 even though anthropogenic inputs of the solvent ceased in 1999.

The most noticeable change between the two periods is the decrease in TCE concentration in well 61BR from $97 \mu \mathrm{g} / \mathrm{L}$ in 2014 to $<1 \mu \mathrm{g} / \mathrm{L}$ in 2016 . This change resulted in a slightly smaller area of vertical TCE contamination along this section between 2014 and 2015-17 (fig. 23).

Most of the wells along section I-I' had TCE concentrations $<1 \mu \mathrm{g} / \mathrm{L}$ in 2014 (fig. 24A) and remained at that level or were not sampled during 2015-17 (fig. 24B). Well 60BR had TCE concentrations that ranged from 560 to $350 \mu \mathrm{g} / \mathrm{L}$, and well 40BR had TCE concentrations that ranged from 5.8 to 9.0 $\mu \mathrm{g} / \mathrm{L}$, over the 4 -year period. The TCE concentrations present in 40BR indicate that TCE contamination was present down to $120 \mathrm{ft}$ below land surface on the western border of the NAWC facility.

Wells along sections $\mathrm{J}-\mathrm{J}^{\prime}$ and $\mathrm{K}-\mathrm{K}^{\prime}$ were not plotted because all samples contained $<1 \mu \mathrm{g} / \mathrm{L}$ TCE. These wells are outside the NAWC site and are primarily on the Mercer County airport property. The fact that these wells all had no TCE contamination indicates that the pump-and-treat system was containing the contaminant plume hydraulically on the western side of the facility.

\section{Vertical Extent of cisDCE Contamination During 2014-17}

The vertical distribution of cisDCE on most cross sections is very similar to the vertical distribution of TCE. The similarity of these distributions is due in large part to the fact that cisDCE is the primary anaerobic biodegradation breakdown product of TCE. Concentrations of cisDCE at greater than the detection levels were present in only 10 wells where TCE concentrations were found to be $<1 \mu \mathrm{g} / \mathrm{L}$. These detections indicate that anaerobic biodegradation had broken down essentially all the TCE in these wells. Concentrations of cisDCE were higher than TCE concentrations in 30 of the 91 wells sampled during 2014-17, indicating active biodegradation was ongoing in at least one-third of the wells at the NAWC site.

\section{Site 3 Eastern Sections A-A' to D-D'}

The vertical extent of cisDCE contamination in wells along section $\mathrm{A}-\mathrm{A}^{\prime}$ in 2014 (fig. 25A) was identical to that of TCE in 2014 (fig. 16A). Low cisDCE concentrations (6.5 and $2.6 \mu \mathrm{g} / \mathrm{L}$ ) were present in wells $11 \mathrm{BR}$ and 50BR down to about $80 \mathrm{ft}$ below land surface (fig. 25A). The main difference between 2014 and 2015-17 is that the cisDCE concentration 


\section{A. 2014 TCE concentrations}

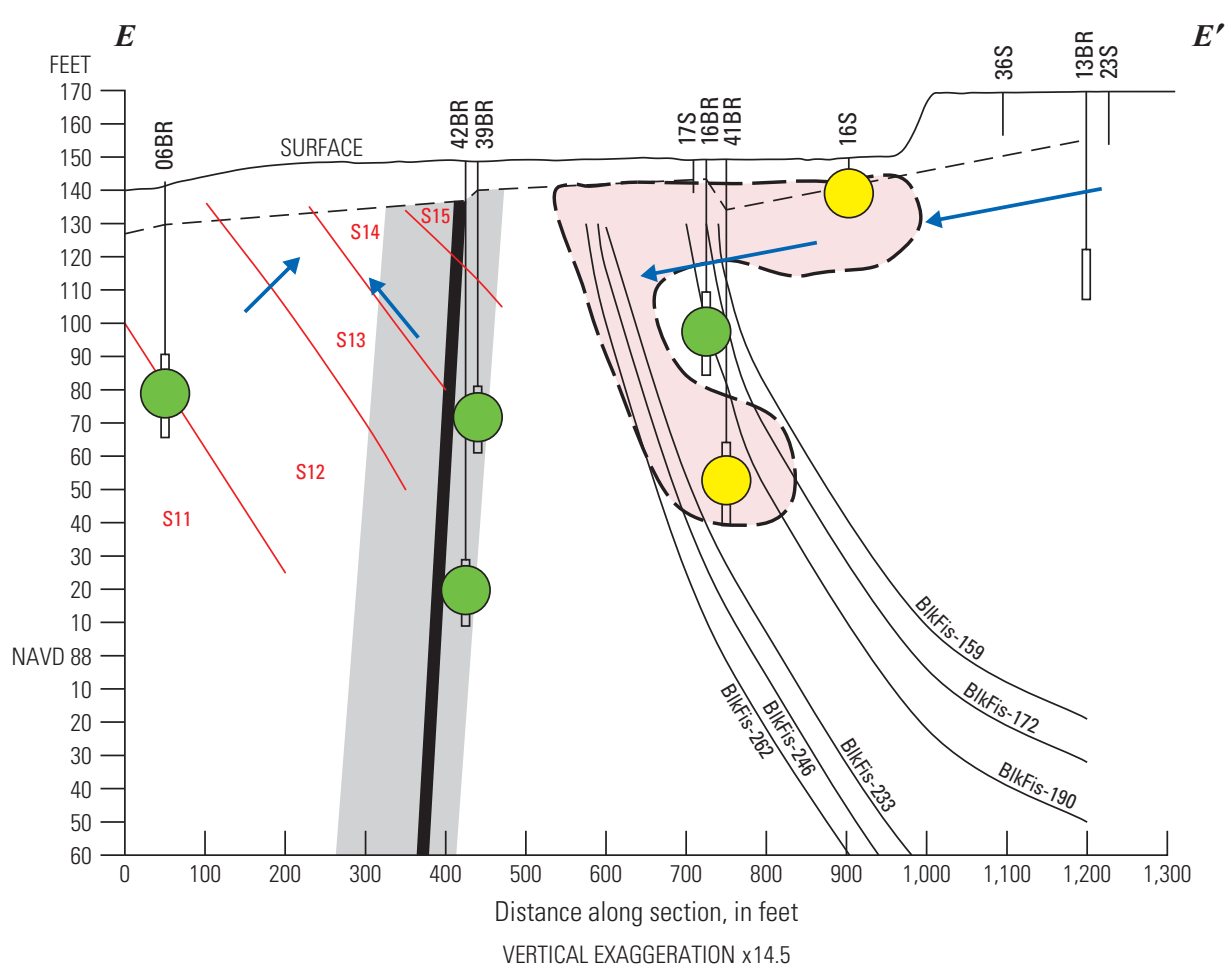

\section{B. 2015-17 TCE most recent concentrations}

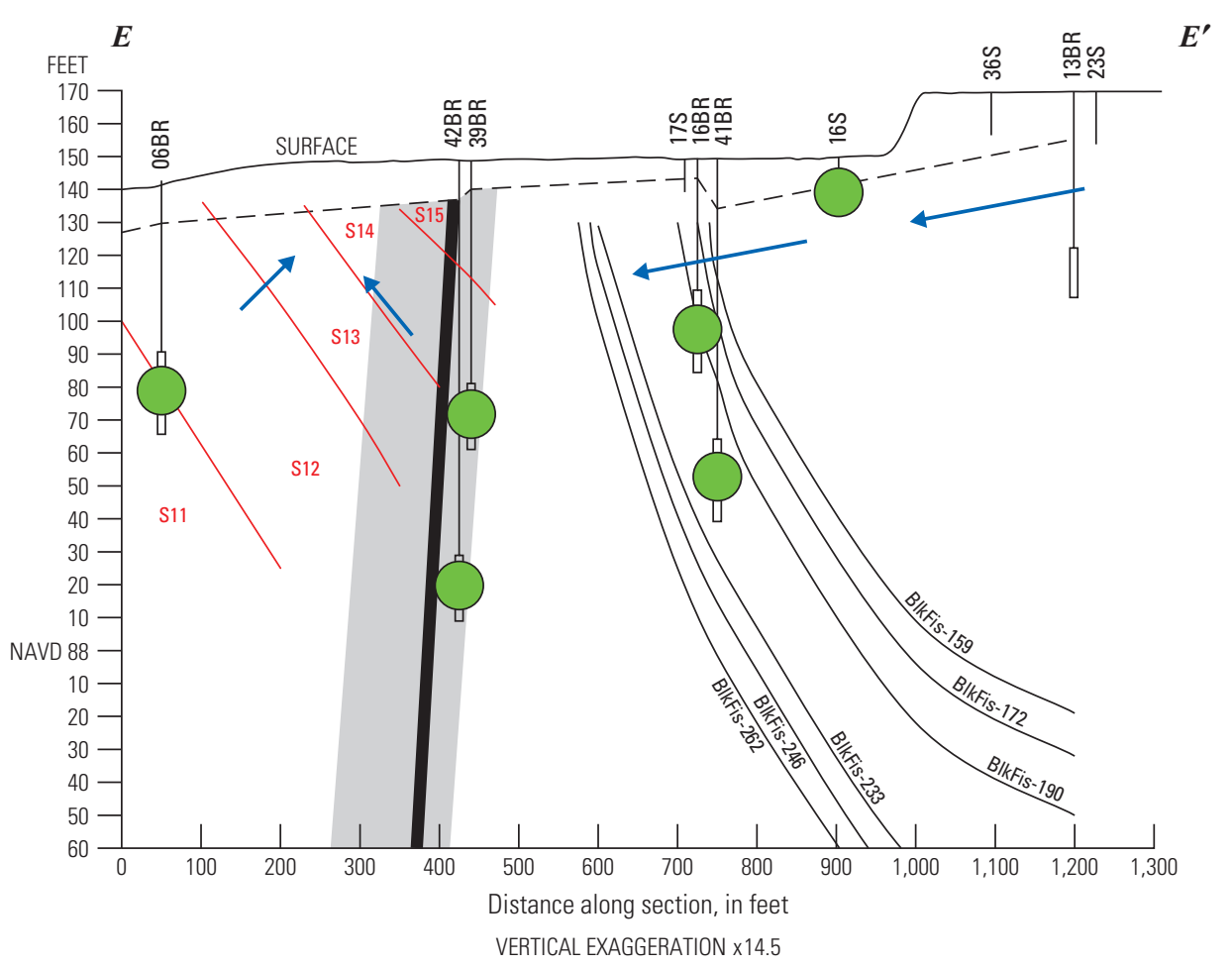

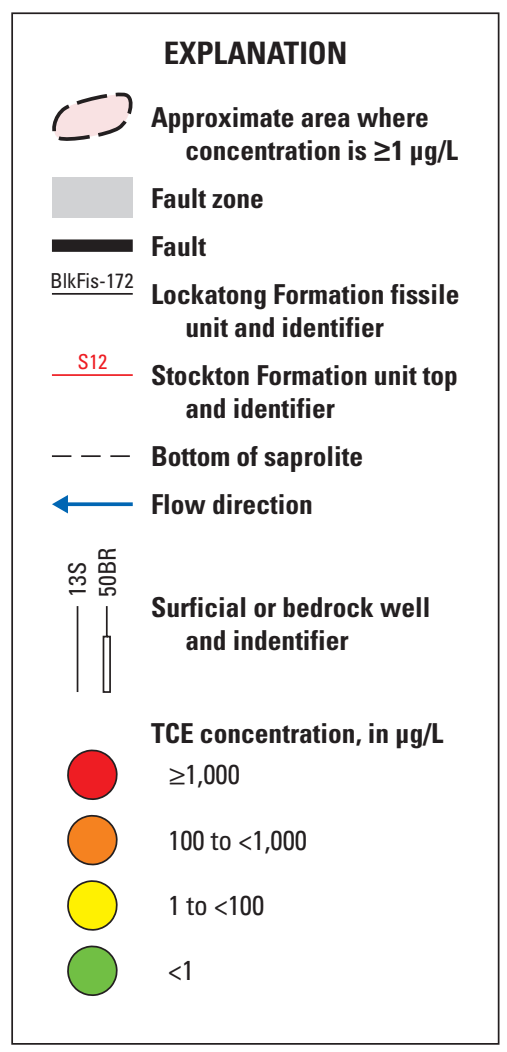

Figure 20. Vertical extent of trichloroethene concentrations in wells along section $\mathrm{E}-\mathrm{E}^{\prime}$ in $A, 2014$ and $B, 2015-17$, former Naval Air Warfare Center, West Trenton, New Jersey. (TCE, trichloroethene; mg/L, micrograms per liter) 


\section{A. 2014 TCE concentrations}

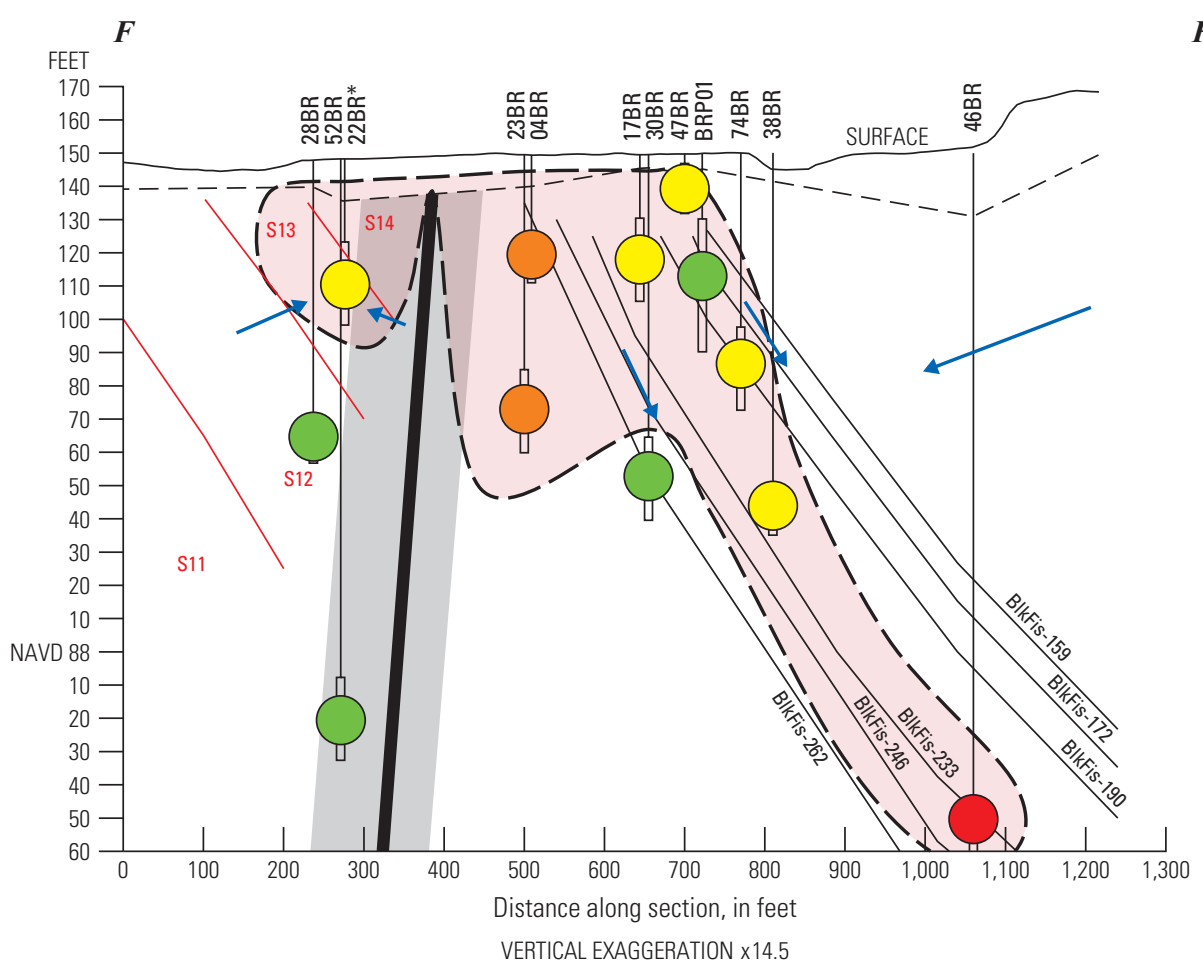

\section{B. 2015-17 TCE most recent concentrations}

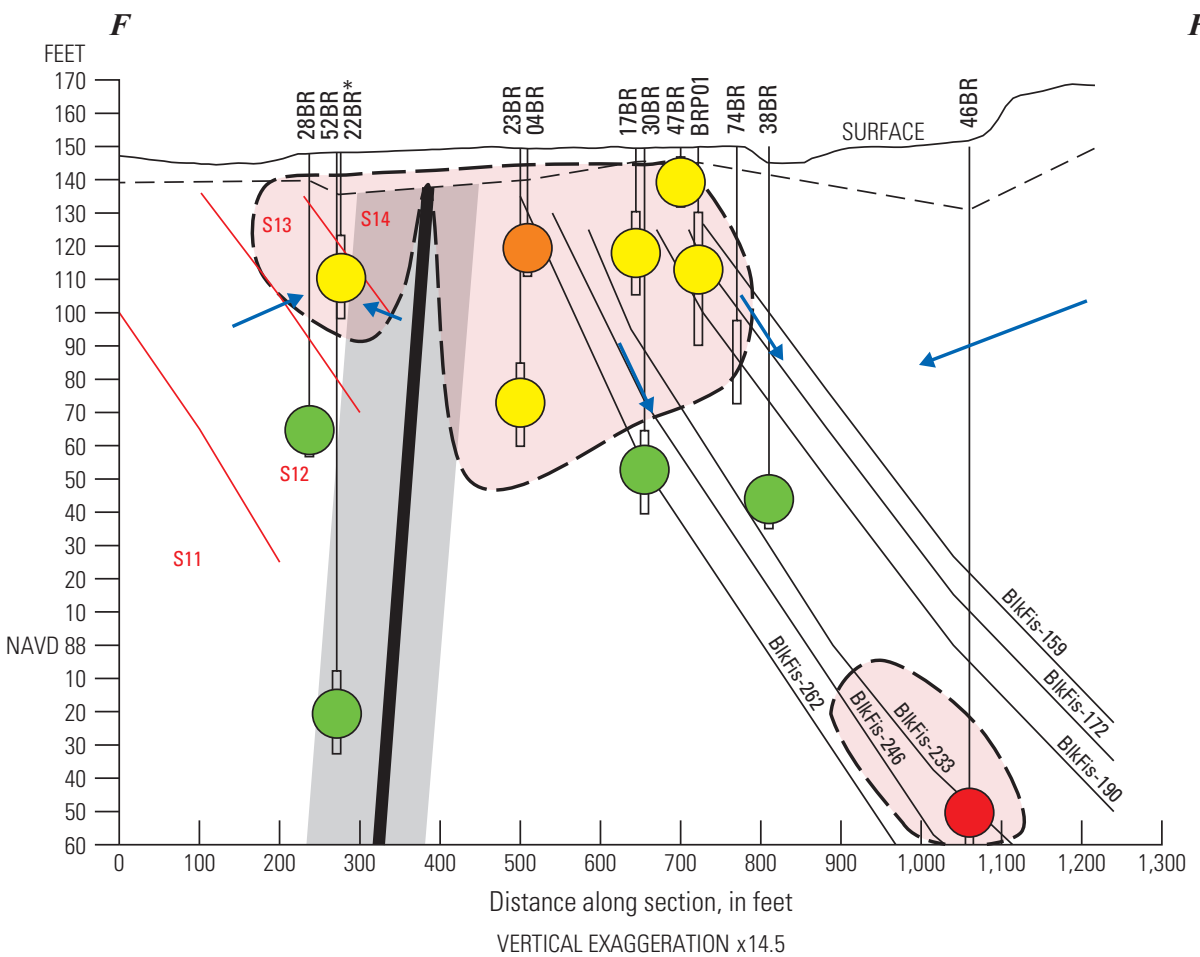

$F^{\prime}$

EXPLANATION
Fpproximate area where
concentration is $\geq 1 \mu \mathrm{g} / \mathrm{L}$
Fault zone
Fault

Figure 21. Vertical extent of trichloroethene concentrations in wells along section $\mathrm{F}-\mathrm{F}^{\prime}$ in $A, 2014$ and $B, 2015-17$, former Naval Air Warfare Center, West Trenton, New Jersey. (TCE, trichloroethene; mg/L, micrograms per liter) 


\section{A. 2014 TCE concentrations}

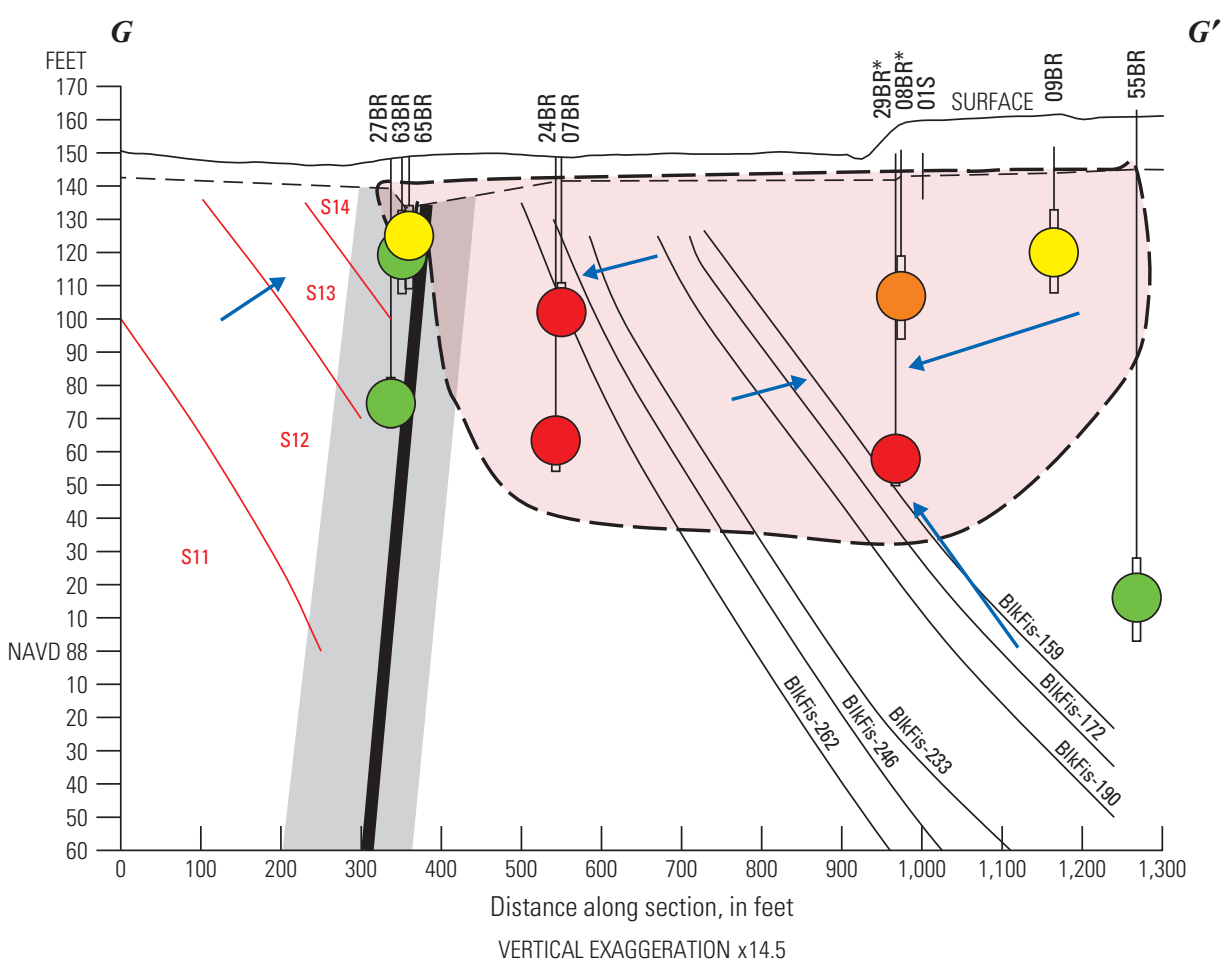

\section{B. 2015-17 TCE most recent concentrations}

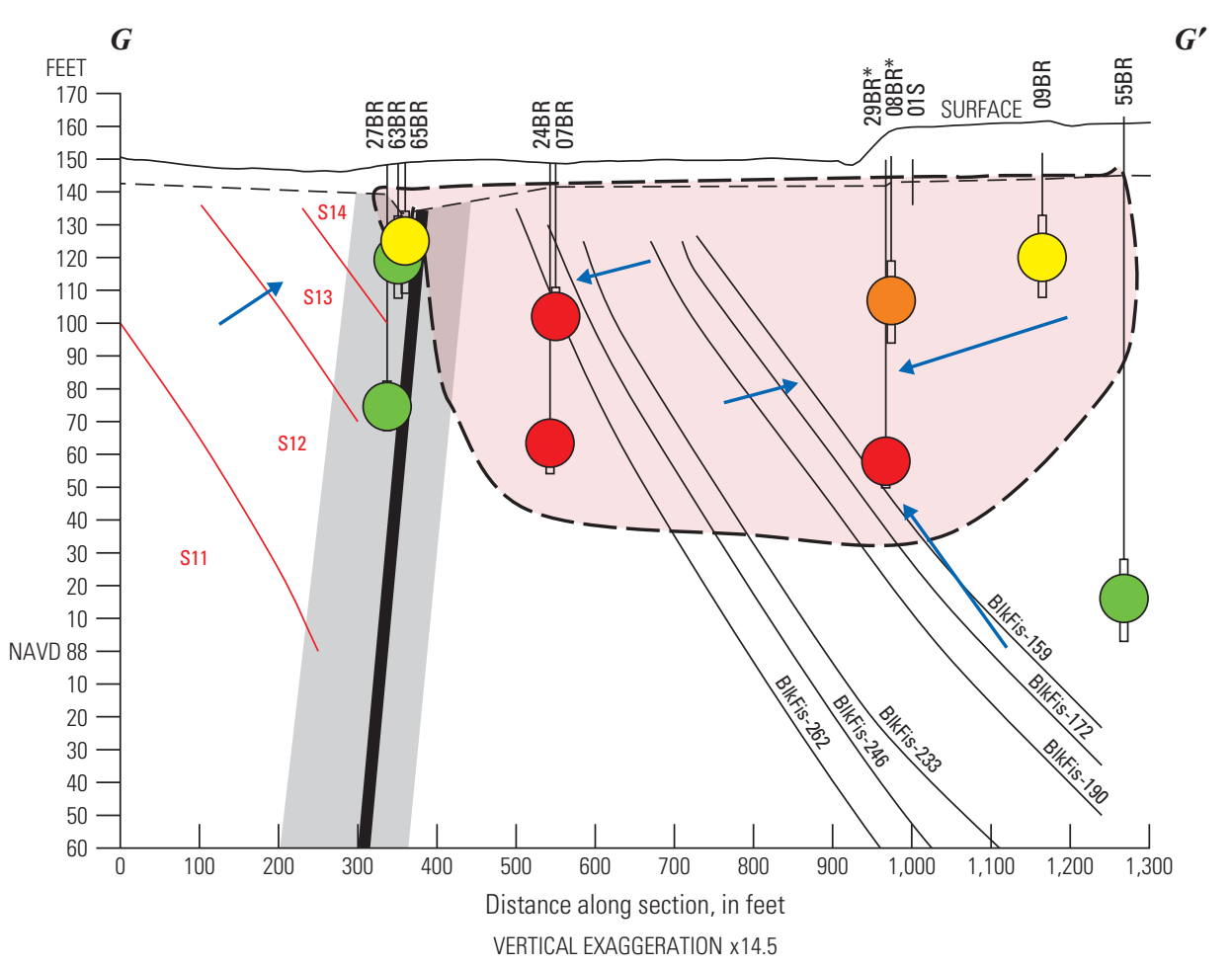

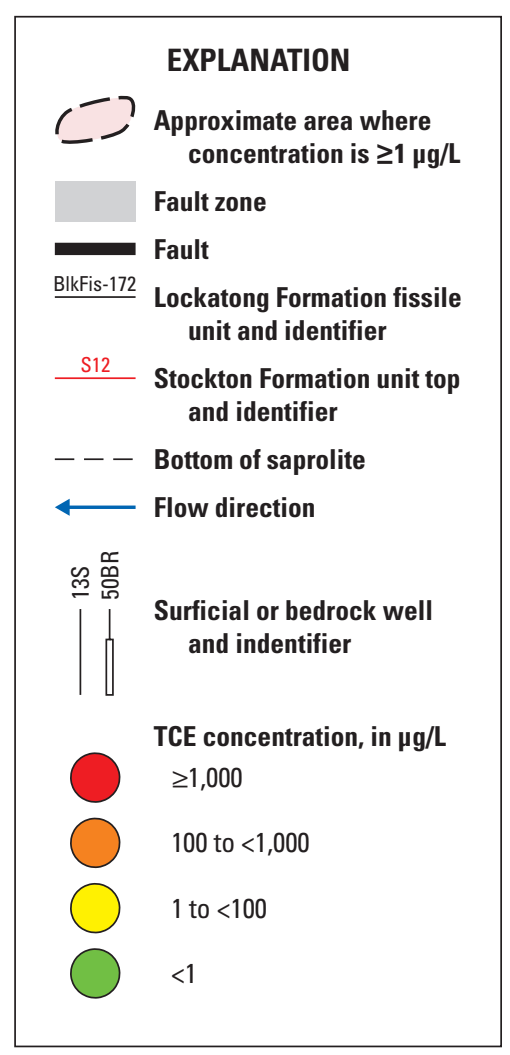

Figure 22. Vertical extent of trichloroethene concentrations in wells along section $\mathrm{G}-\mathrm{G}$ ' in $A, 2014$ and $B, 2015-17$, former Naval Air Warfare Center, West Trenton, New Jersey. (TCE, trichloroethene; mg/L, micrograms per liter) 


\section{A. 2014 TCE concentrations}

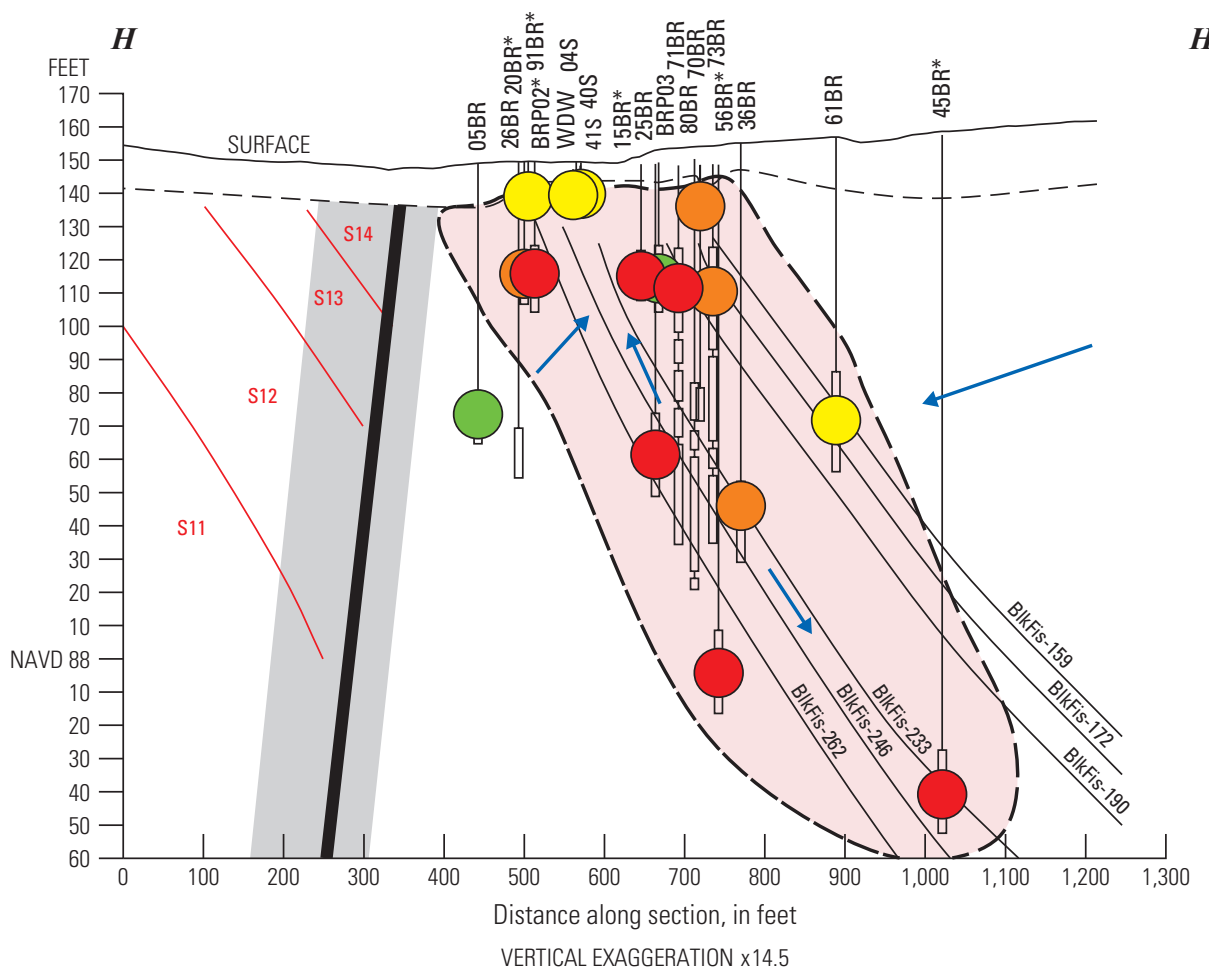

\section{B. 2015-17 TCE most recent concentrations}

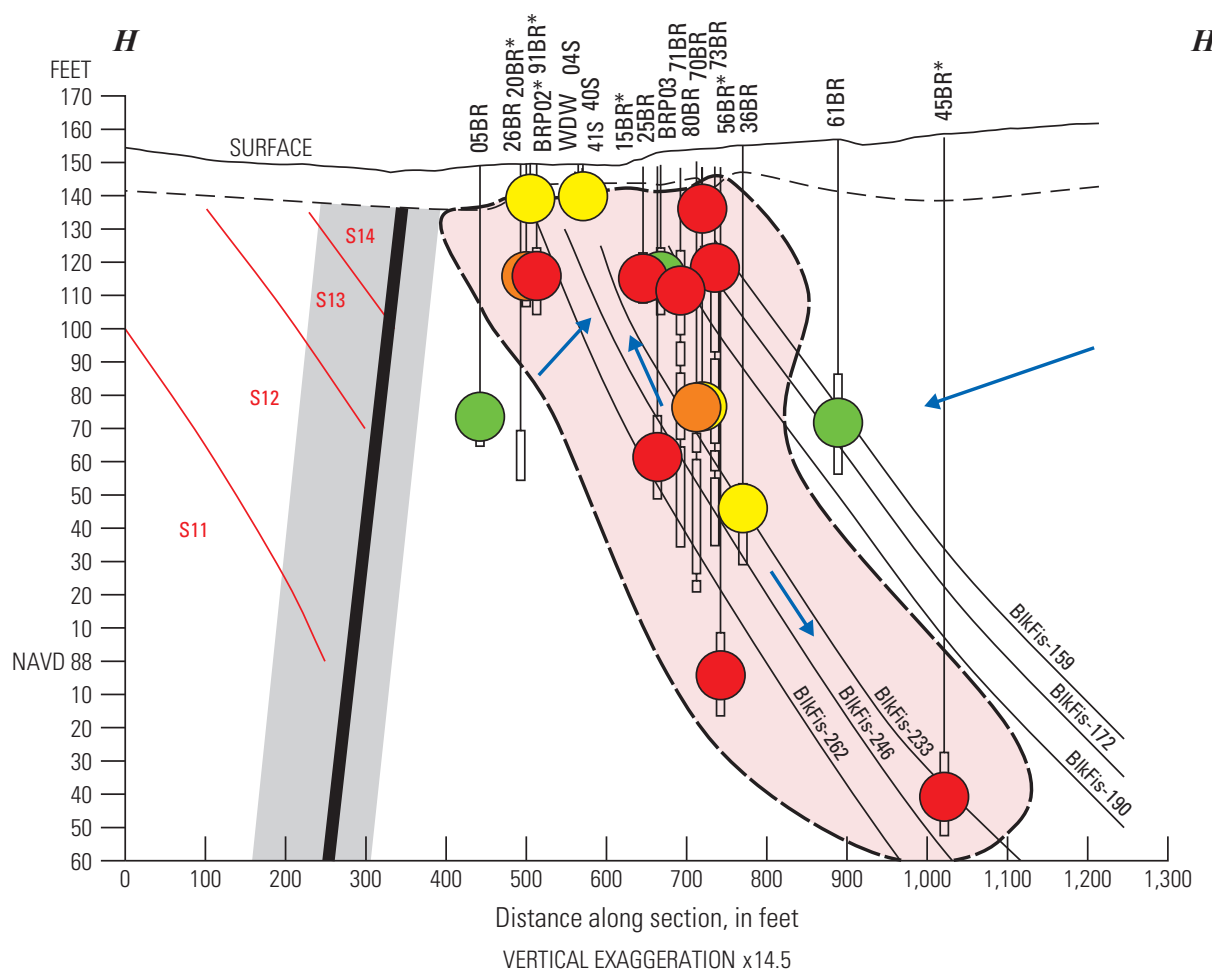

$\boldsymbol{H}^{\prime}$

\begin{tabular}{|c|c|}
\hline & EXPLANATION \\
\hline & $\begin{array}{l}\text { Approximate area where } \\
\text { concentration is } \geq 1 \mu \mathrm{g} / \mathrm{L}\end{array}$ \\
\hline & Fault zone \\
\hline & Fault \\
\hline & $\begin{array}{l}\text { Lockatong Formation fissile } \\
\text { unit and identifier }\end{array}$ \\
\hline & $\begin{array}{l}\text { Stockton Formation unit top } \\
\text { and identifier }\end{array}$ \\
\hline & Bottom of saprolite \\
\hline & Flow direction \\
\hline & $\begin{array}{l}\text { Surficial or bedrock well } \\
\text { and indentifier }\end{array}$ \\
\hline & $\begin{array}{l}\text { TCE concentration, in } \mu \mathrm{g} / \mathrm{L} \\
\geq 1,000\end{array}$ \\
\hline & 100 to $<1,000$ \\
\hline & 1 to $<100$ \\
\hline & $<1$ \\
\hline
\end{tabular}

Figure 23. Vertical extent of trichloroethene concentrations in wells along section $\mathrm{H}-\mathrm{H}^{\prime}$ in $A, 2014$ and $B, 2015-17$, former Naval Air Warfare Center, West Trenton, New Jersey. (TCE, trichloroethene; mg/L, micrograms per liter) 


\section{A. 2014 TCE concentrations}

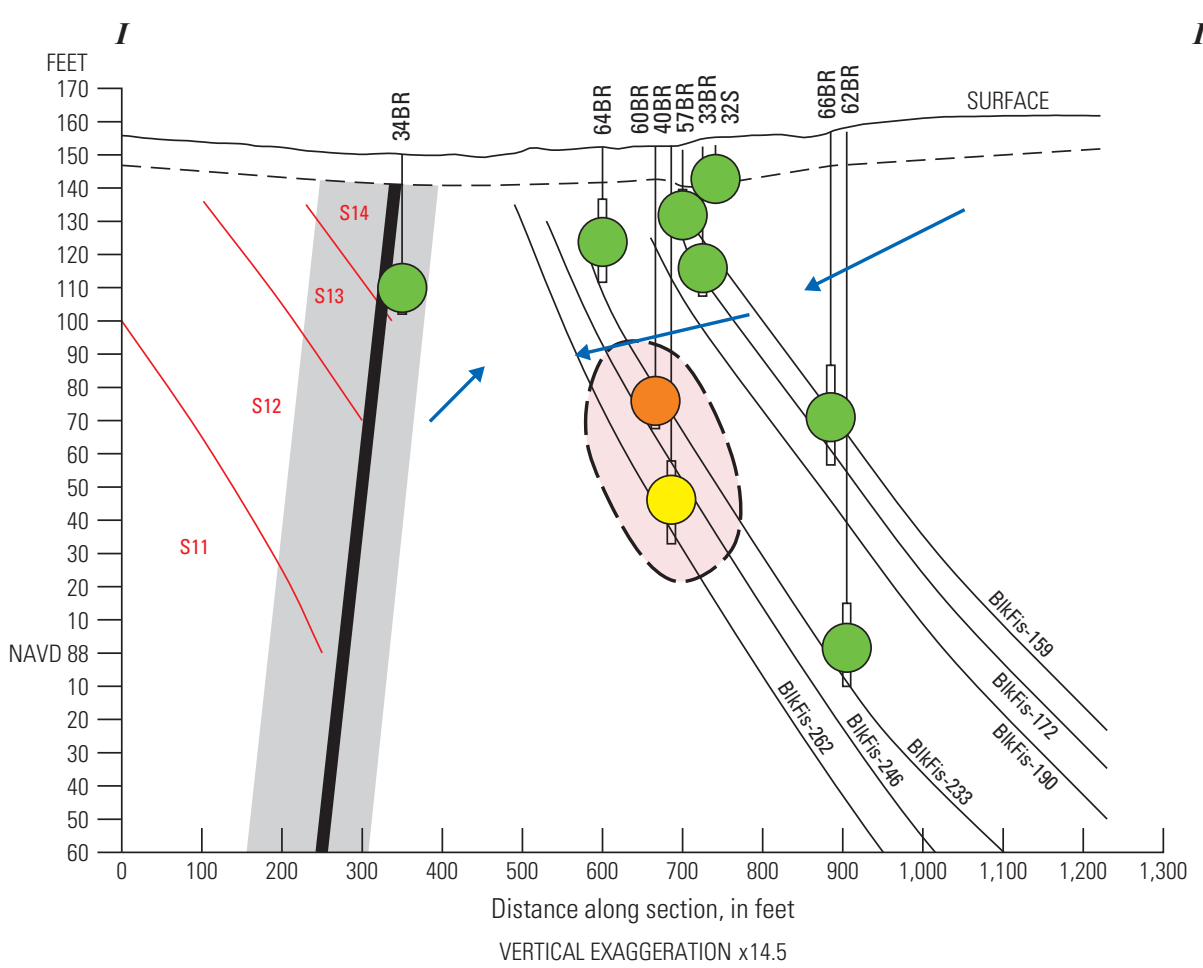

\section{B. 2015-17 TCE most recent concentrations}

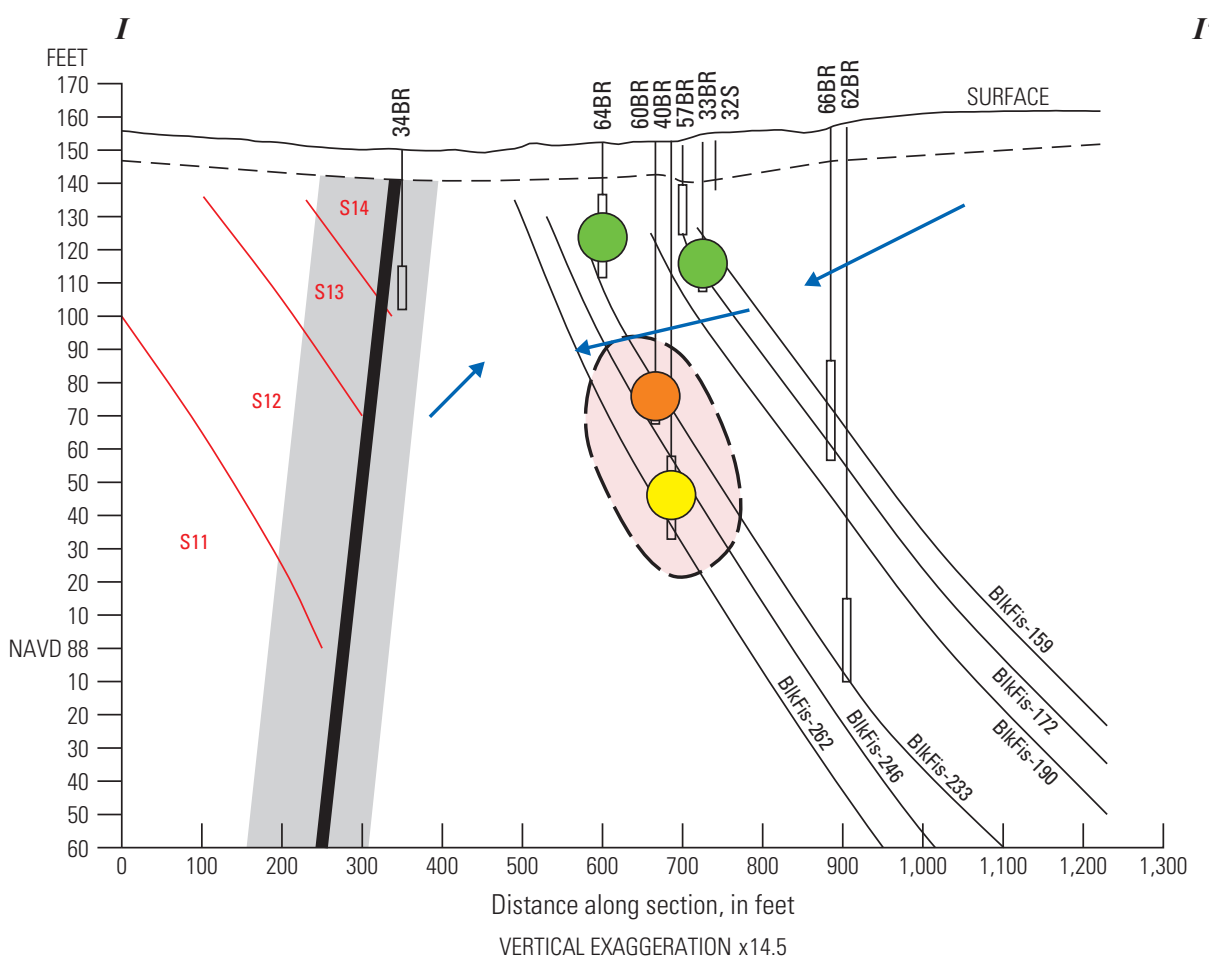

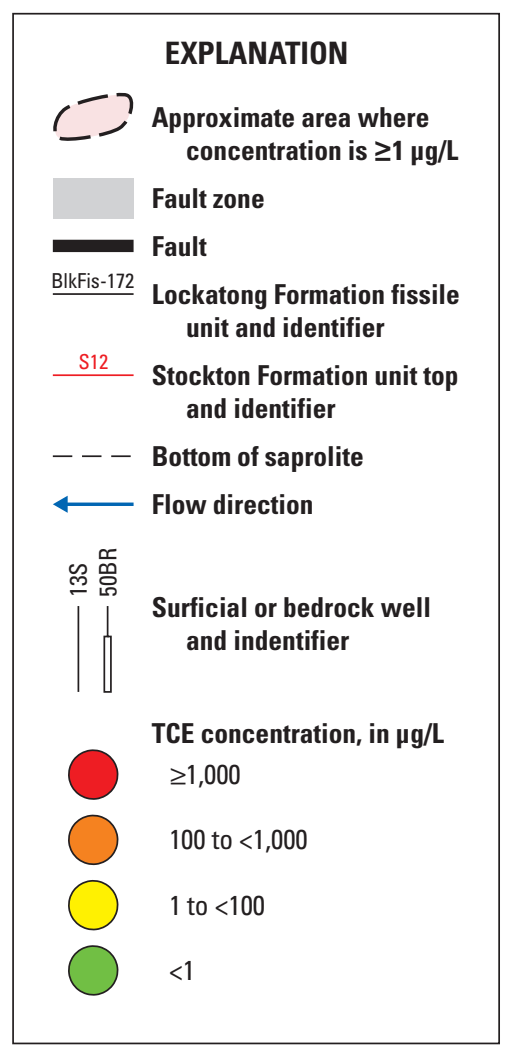

Figure 24. Vertical extent of trichloroethene concentrations in wells along section I-I' in A, 2014 and B, 2015-17, former Naval Air Warfare Center, West Trenton, New Jersey. (TCE, trichloroethene; mg/L, micrograms per liter) 
in well 11BR decreased from $6.5 \mu \mathrm{g} / \mathrm{L}$ in 2014 to $<1.1 \mu \mathrm{g} / \mathrm{L}$ in 2016 (fig. 25B). This slight decrease reduced the shaded area of vertical contamination on this section substantially.

The vertical extent of cisDCE contamination along section B-B' (fig.26A) was more widespread than that of TCE in 2014 (fig. 26A and 17A). Concentrations of cisDCE $\geq 1$ $\mu \mathrm{g} / \mathrm{L}$ were present in five wells to a maximum depth of $96 \mathrm{ft}$ (fig. 26A) in 2014. Four of these wells had cisDCE concentrations between 2.0 and $6.9 \mu \mathrm{g} / \mathrm{L}$, whereas well $02 \mathrm{BR}$ had the highest cisDCE concentration $(33 \mu \mathrm{g} / \mathrm{L})$ of any wells along this section. The difference between the 2014 data (fig. 26A) and the 2015-17 data (fig. 26B) was that the concentration in well $12 \mathrm{~S}$ decreased to $<0.6 \mu \mathrm{g} / \mathrm{L}$ and well $28 \mathrm{~S}$ was not sampled. Therefore, the vertical extent of the cisDCE contamination was smaller in the most recent samples.

The vertical extent of detectable cisDCE concentrations along section $\mathrm{C}-\mathrm{C}^{\prime}$ ranged from shallow groundwater depths in well $31 \mathrm{~S}$, to $200 \mathrm{ft}$ below land surface in well 54BR (fig. 27A). This vertical distribution is very similar to the vertical distribution of TCE along this section (fig. 18). The vertical extent of cisDCE concentrations in wells along section C-C' was essentially unchanged between 2014 and 2015-17 (fig 27A and fig 27B). Wells 31BR, 31S, 48BR, and 54BR had cisDCE concentrations between $1.6 \mu \mathrm{g} / \mathrm{L}$ and $37 \mu \mathrm{g} / \mathrm{L}$ during this 4-year period.

No cisDCE concentrations $\geq 1 \mu \mathrm{g} / \mathrm{L}$ were present in samples from wells along section D-D' during 2014 or 2015-17 (fig. 28). This matches the absence of detectable TCE in wells along this section observed previously (fig. 19).

\section{Site 1 Western Sections E-E' to I-I'}

Most of the wells along section E-E' in 2014 were characterized by low cisDCE concentrations (fig. 29A); only wells 16BR and $41 \mathrm{BR}$ had concentrations $\geq 1 \mu \mathrm{g} / \mathrm{L}$. The detectable cisDCE concentrations extended from shallow groundwater to $110 \mathrm{ft}$ below land surface in well 41BR (fig 29A). The main difference from 2014 to $2015-17$ is that the concentration in well 41BR decreased from $3.9 \mu \mathrm{g} / \mathrm{L}$ to $<0.41 \mu \mathrm{g} / \mathrm{L}$. Therefore, the vertical extent of the shaded area of cisDCE contamination is smaller for 2015-17 (fig. 29B).

Most wells along section F-F' were contaminated with cisDCE in 2014 and 2015-17. The vertical distribution of cisDCE concentrations $\geq 1 \mu \mathrm{g} / \mathrm{L}$ in wells along this section was from the shallow groundwater down to $221 \mathrm{ft}$ into the bedrock aquifer in well 46BR (fig. 30). The vertical extent of cisDCE contamination did not change from 2014 to 2015-17. The highest cisDCE concentrations along this section were present in wells 04BR, 38BR, and 74BR, which had concentrations from 100 to $<1,000 \mu \mathrm{g} / \mathrm{L}$ in 2014 (fig. 30A). The main difference between the periods is that well 74BR was not sampled during 2015-17 (fig. 30B). The cisDCE concentration in 38BR decreased substantially from 780 to $320 \mu \mathrm{g} / \mathrm{L}$, and in 46BR decreased from 96 to $13 \mu \mathrm{g} / \mathrm{L}$ over the 4 -year period.
The vertical distribution of cisDCE concentrations in wells along section $\mathrm{G}-\mathrm{G}^{\prime}$ was from the shallow groundwater to at least $100 \mathrm{ft}$ below land surface in wells 24BR and 29BR (fig. 31). The vertical extent of cisDCE contamination did not change from 2014 (fig 31A) to 2015-17 (fig 31B). Wells 07BR and 24BR were the two wells most contaminated with cisDCE; concentrations ranged from 4,300 to $10,000 \mu \mathrm{g} / \mathrm{L}$ over the 4-year period. These two wells are in the center of the Site 1 area where much of the TCE was originally spilled or leaked. The high concentrations of cisDCE indicate TCE biodegradation has been stimulated in the aquifer at these wells.

Along Section $\mathrm{H}-\mathrm{H}^{\prime}$, concentrations of cisDCE $\geq 1 \mu \mathrm{g} / \mathrm{L}$ extended vertically from shallow groundwater to $210 \mathrm{ft}$ below land surface in well 45BR (fig. 32). This section includes many of the most contaminated wells at the former NAWC site. Of the 16 wells sampled along this section in 2014, nine had cisDCE concentrations $\geq 1,000 \mu \mathrm{g} / \mathrm{L}$ (red dots), and 5 wells had cisDCE concentrations from 100 to $<1,000 \mu \mathrm{g} / \mathrm{L}$ (orange dots) (fig. 32A). These high cisDCE concentrations indicate there was a great deal of anaerobic biodegradation of TCE going on in this area. Some biodegradation was due to the 2008 bioaugmentation project that occurred between 2008 and 2013 in wells along this section (Shapiro and others, 2018), but most of the biodegradation in other wells was undoubtedly due to the activity of indigenous dechlorinating bacteria at the site. The major differences between the plots for 2014 (fig. 32A) and 2015-17 (fig. 32B) are that cisDCE concentrations in well 45BR decreased from 2,100 to $930 \mu \mathrm{g} / \mathrm{L}$ and in well 61BR decreased from 150 to $9.4 \mu \mathrm{g} / \mathrm{L}$. The overall shaded vertical area of cisDCE contamination, however, did not change over the 4-year period.

The vertical distribution of cisDCE in wells along Section I-I' extended from shallow groundwater to $120 \mathrm{ft}$ below land surface in well 40BR (fig. 33). In 2014, wells 33BR, 40BR, 60BR, and 64BR had cisDCE concentrations from 1 to $<100$ $\mu \mathrm{g} / \mathrm{L}$ (yellow dots) (fig. 33A). During 2015-17, cisDCE concentrations remained the same in 33BR and 64BR but increased slightly in 60BR (from 38 to $96 \mu \mathrm{g} / \mathrm{L}$ ) and 40BR (from 72 to $130 \mu \mathrm{g} / \mathrm{L}$ ) (fig. 33B). These increases in cisDCE were most likely due to increased biodegradation of TCE in the aquifer at these wells. The overall extent of cisDCE contamination $\geq 1 \mu \mathrm{g} / \mathrm{L}$ did not change from 2014 to 2017 .

\section{Vertical Extent of VC Contamination During 2014-17}

The vertical distribution of $\mathrm{VC}$ concentrations along most lines of section were similar to the vertical distribution of cisDCE discussed previously. The similarity of these distributions is because $\mathrm{VC}$ is the biological breakdown product of cisDCE. In most wells at NAWC, VC was not the VOC found in the highest concentration because there is usually a "stall" in the anaerobic biodegradation of TCE during the step where cisDCE is degraded to VC (Wiedemeier and 


\section{A. 2014 cisDCE concentrations}

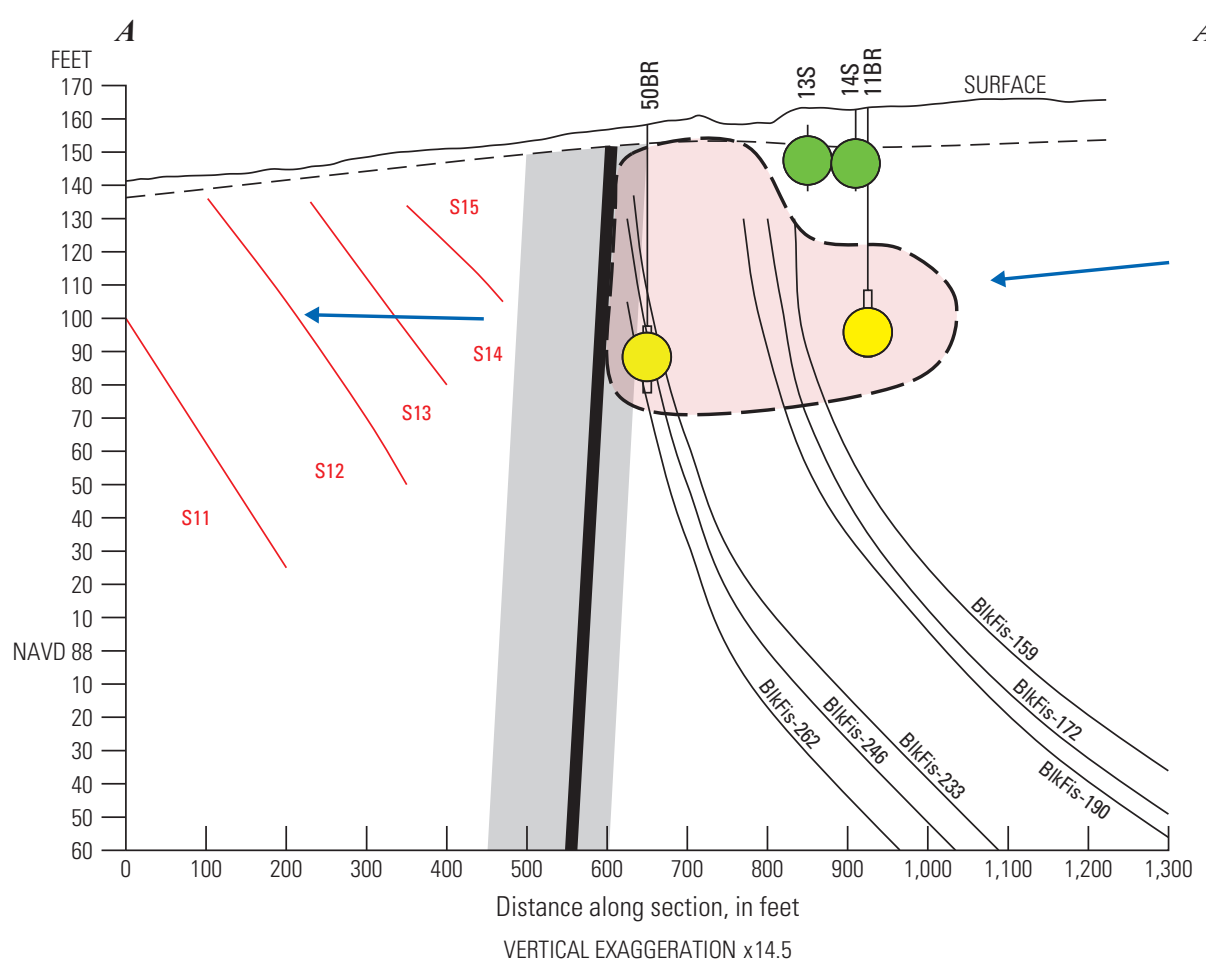

\section{B. 2015-17 cisDCE most recent concentrations}

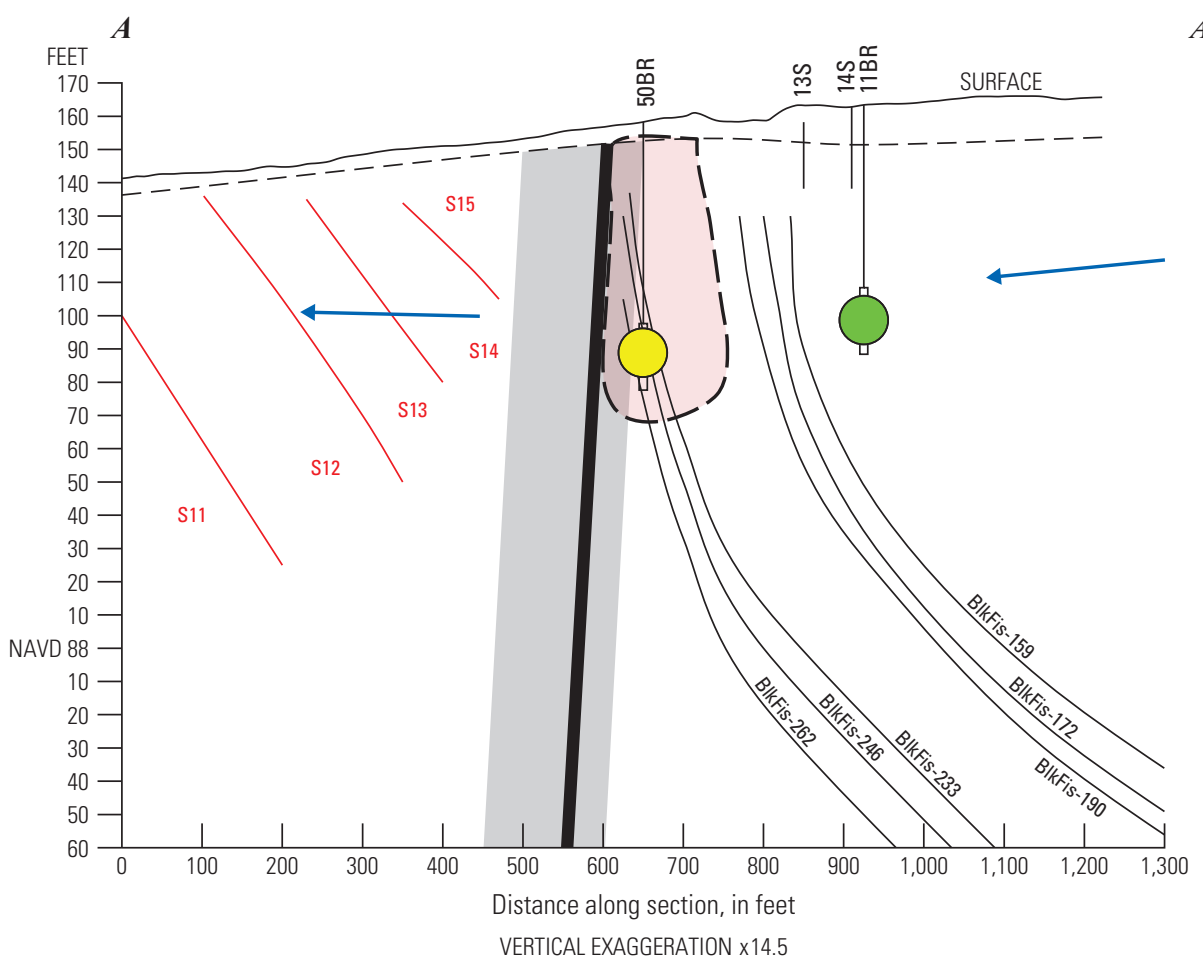

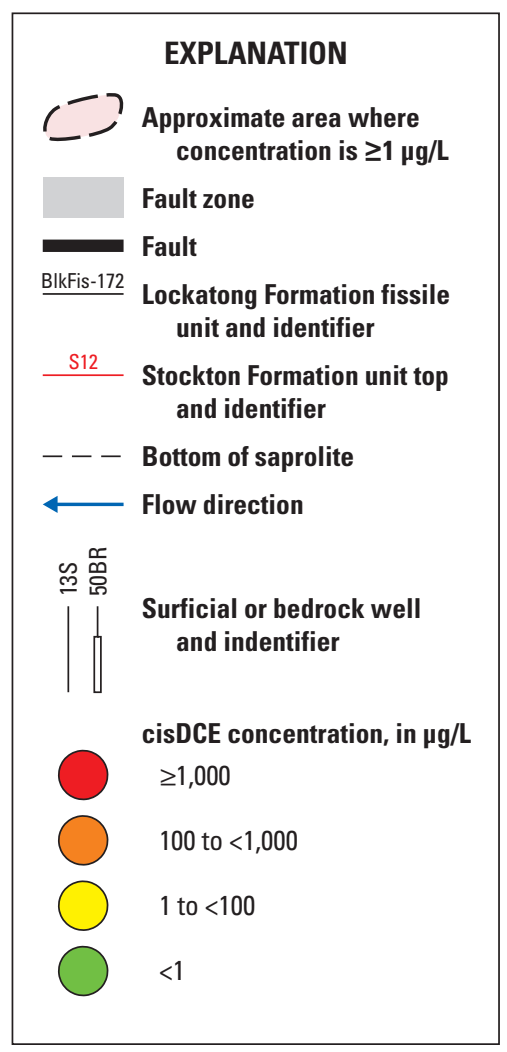

Figure 25. Vertical extent of cis-1,2-dichloroethene concentrations in wells along section A-A' in A, 2014 and B, 2015-17, former Naval Air Warfare Center, West Trenton, New Jersey. (cisDCE, cis-1,2-dichloroethene; mg/L, micrograms per liter) 


\section{A. 2014 cisDCE concentrations}

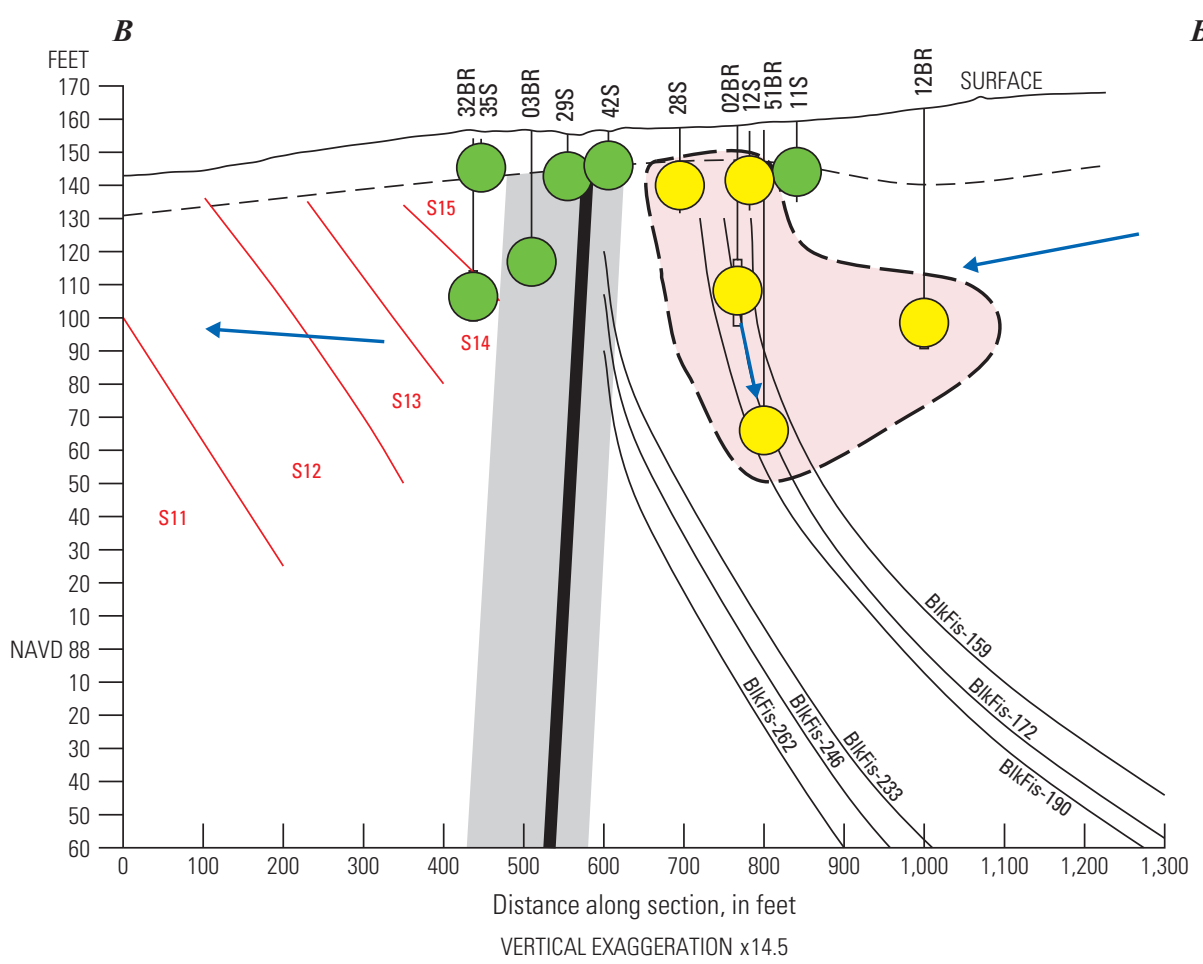

\section{B. 2015-17 cisDCE most recent concentrations}

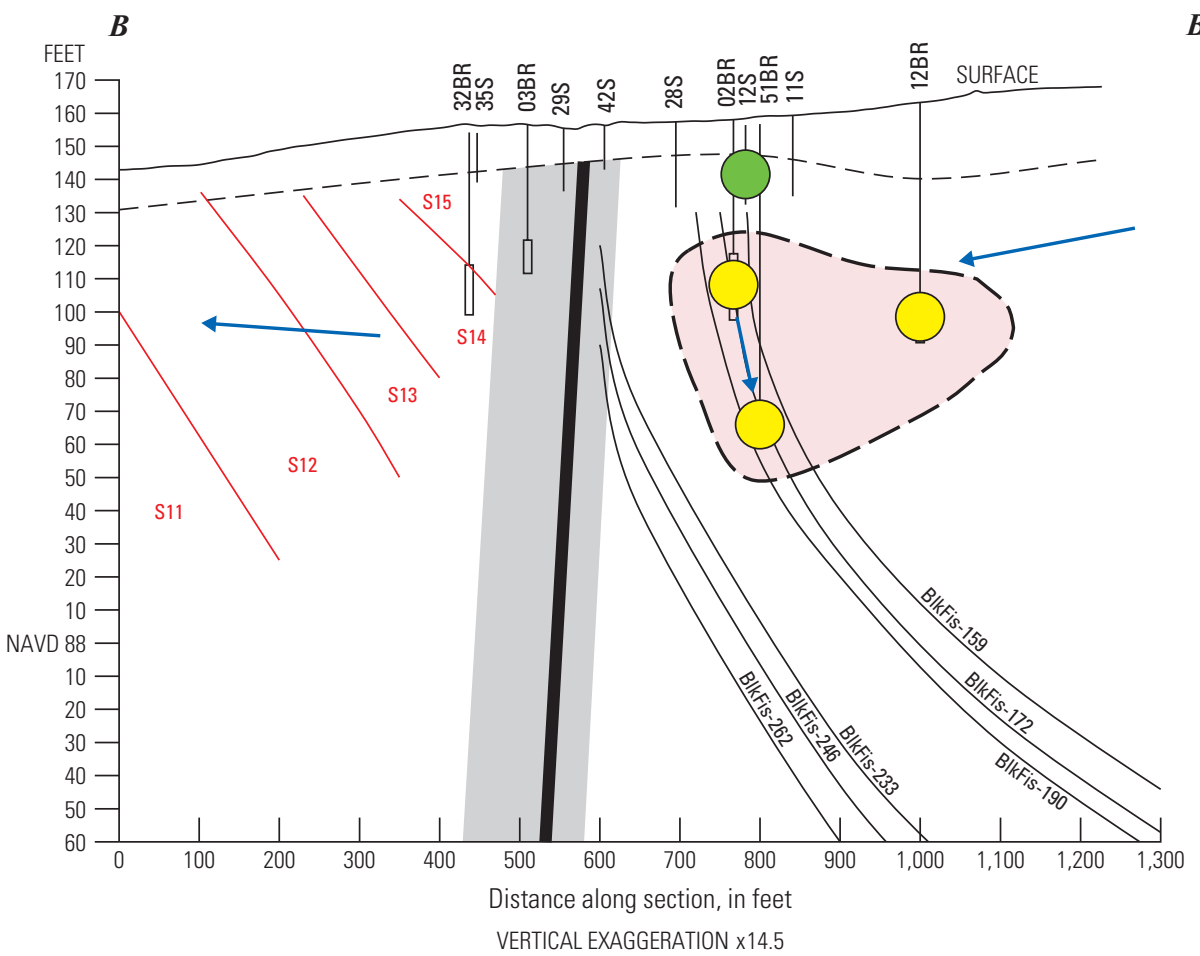

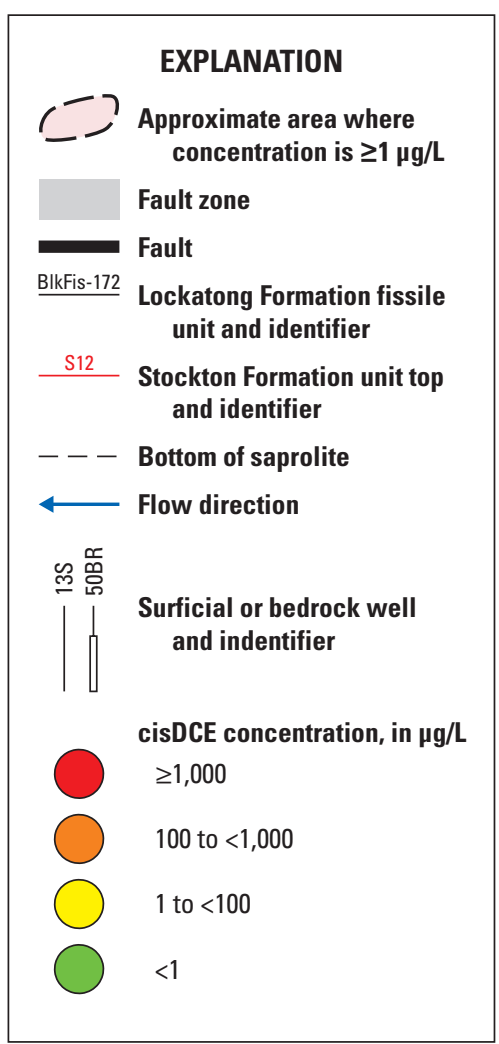

Figure 26. Vertical extent of cis-1,2-dichloroethene concentrations in wells along section $B-B$ ' in $A, 2014$ and $B, 2015-17$, former Naval Air Warfare Center, West Trenton, New Jersey. (cisDCE, cis-1,2-dichloroethene; mg/L, micrograms per liter) 


\section{A. 2014 cisDCE concentrations}

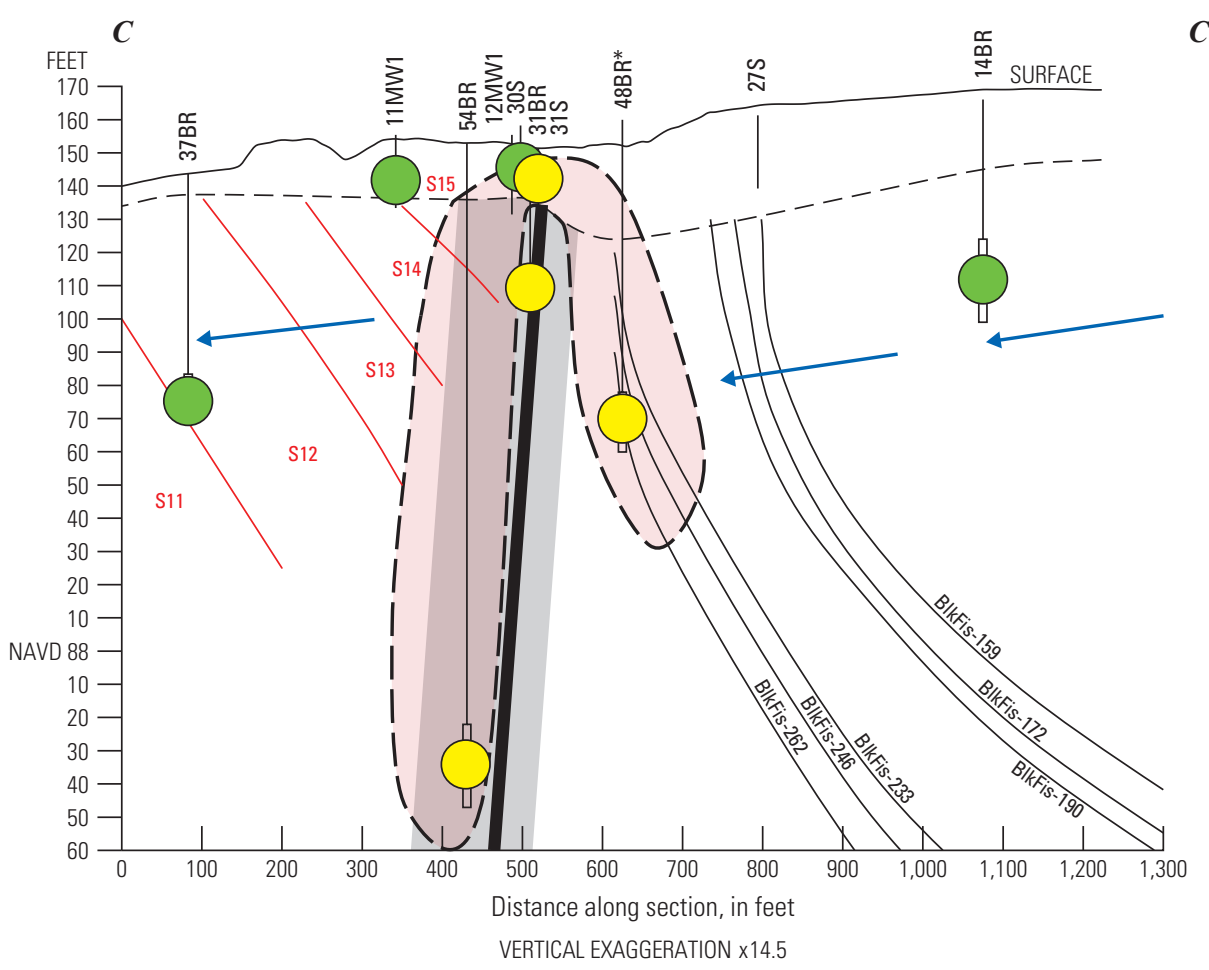

\section{B. 2015-17 cisDCE most recent concentrations}

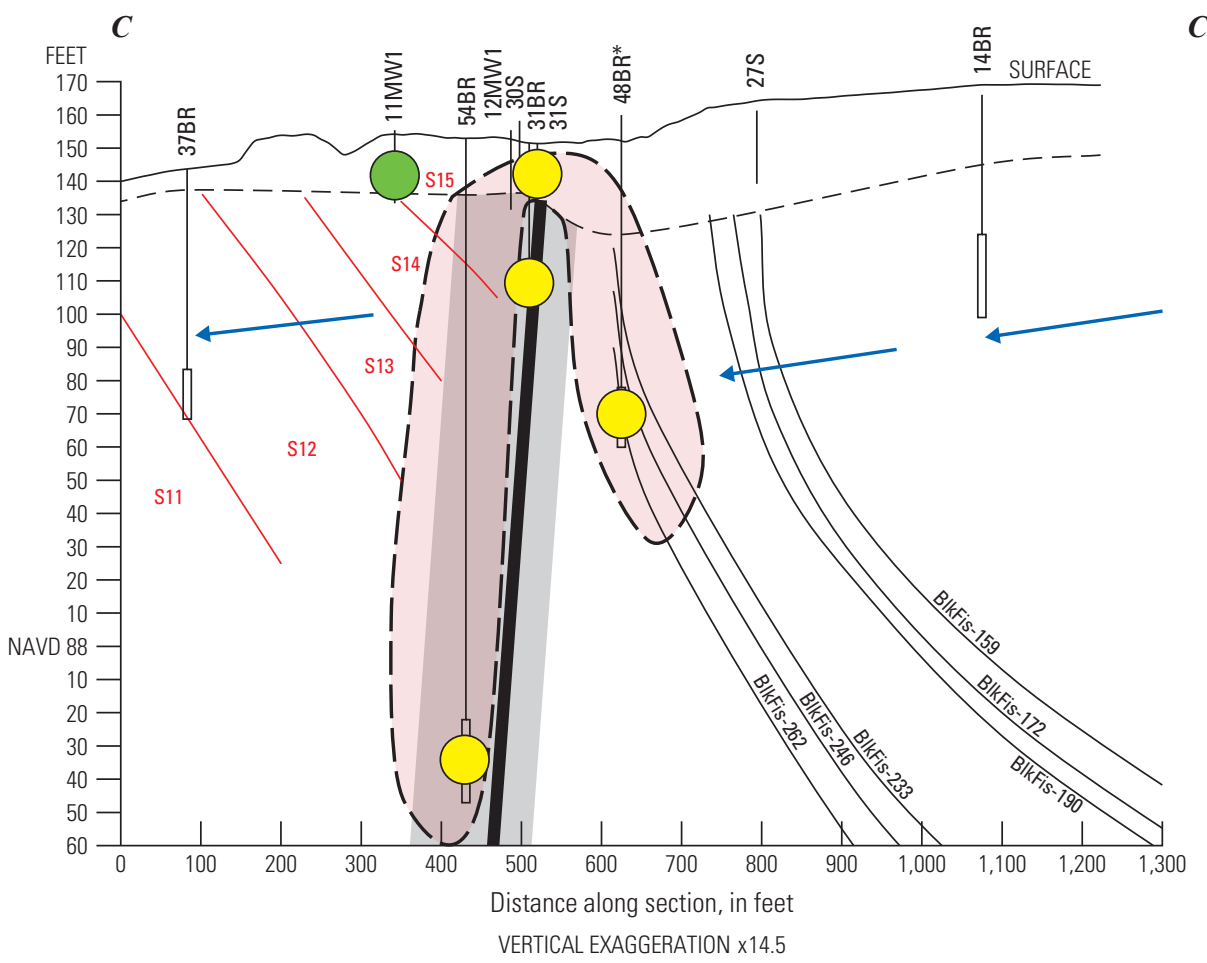

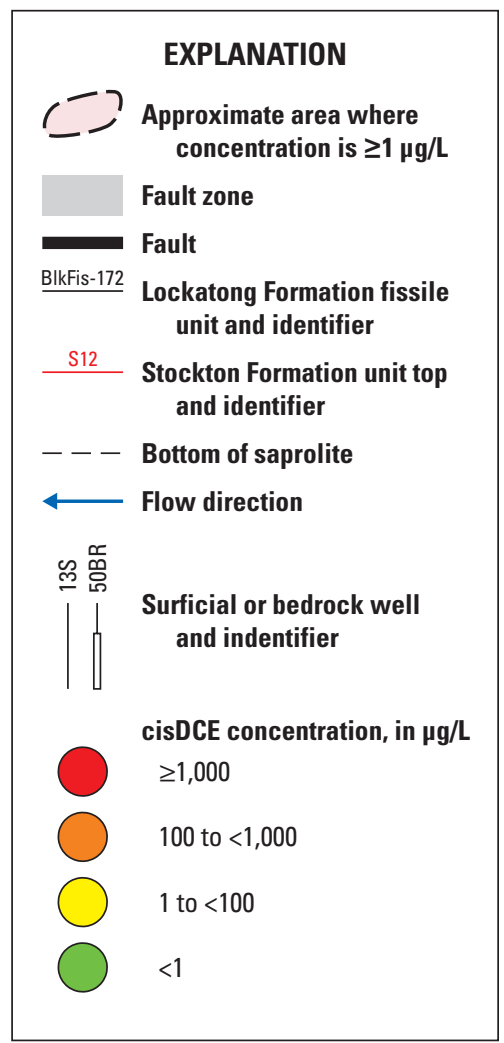

Figure 27. Vertical extent of cis-1,2-dichloroethene concentrations in wells along section $C-C$ ' in $A, 2014$ and $B, 2015-17$, former Naval Air Warfare Center, West Trenton, New Jersey. (cisDCE, cis-1,2-dichloroethene; mg/L, micrograms per liter) 


\section{A. 2014 cisDCE concentrations}

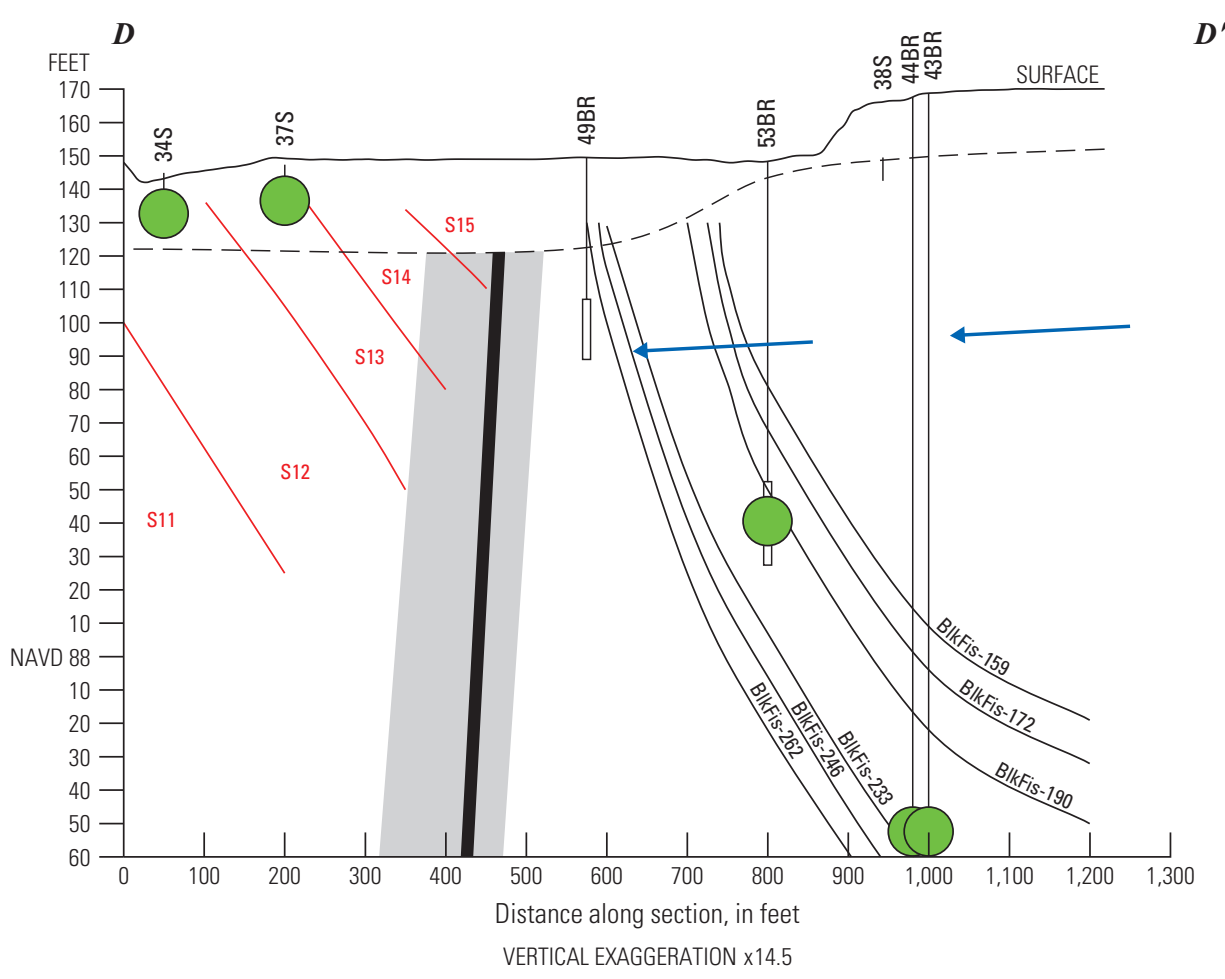

\section{B. 2015-17 cisDCE most recent concentrations}

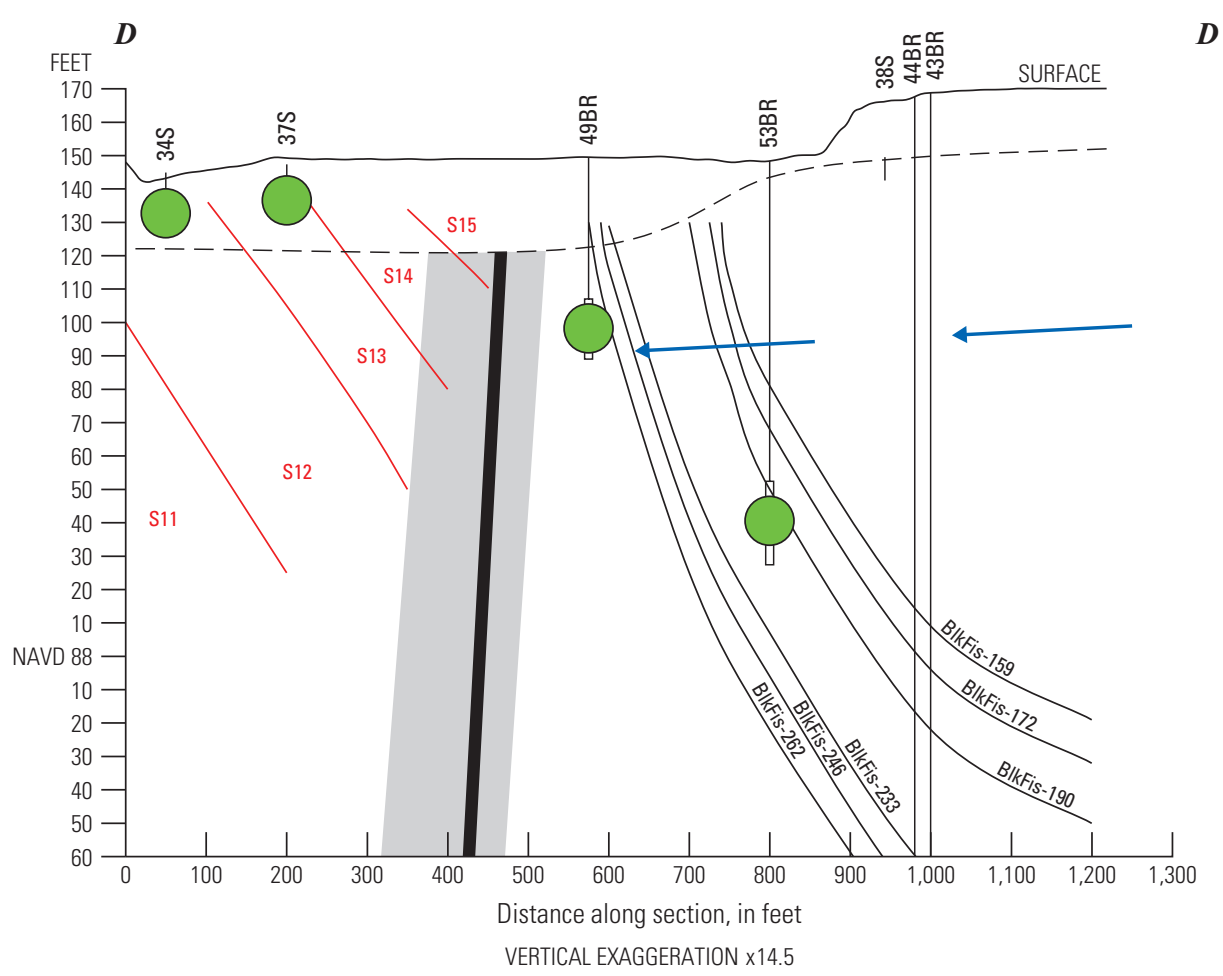

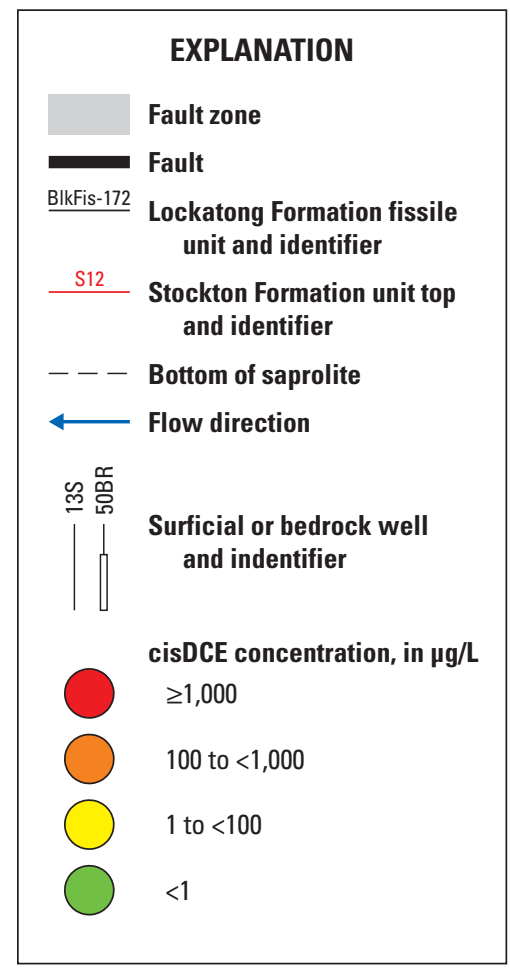

Figure 28. Vertical extent of cis-1,2-dichloroethene concentrations in wells along section D-D' in A, 2014 and B, 2015-17, former Naval Air Warfare Center, West Trenton, New Jersey. (cisDCE, cis-1,2-dichloroethene; mg/L, micrograms per liter) 


\section{A. 2014 cisDCE concentrations}

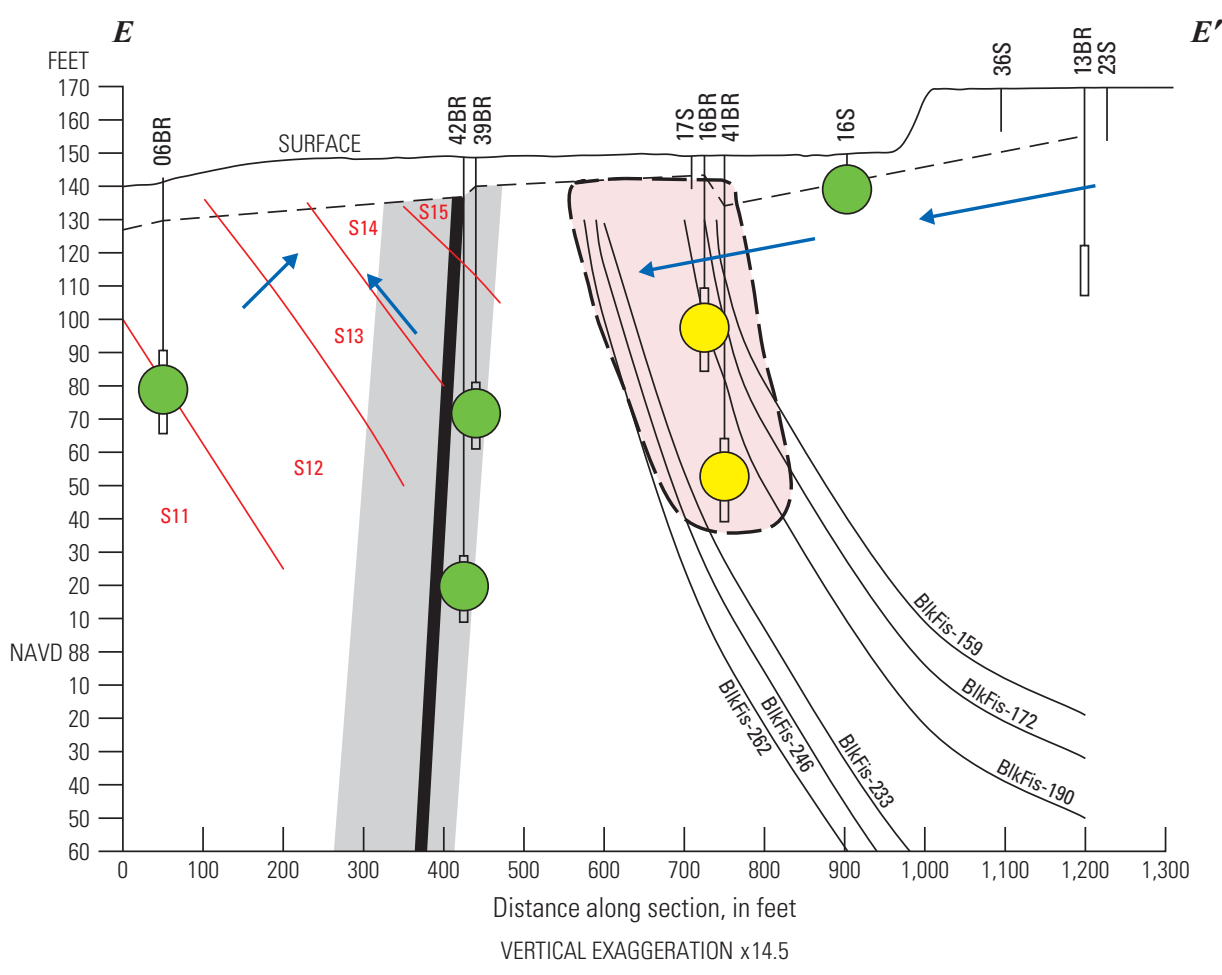

\section{B. 2015-17 cisDCE most recent concentrations}

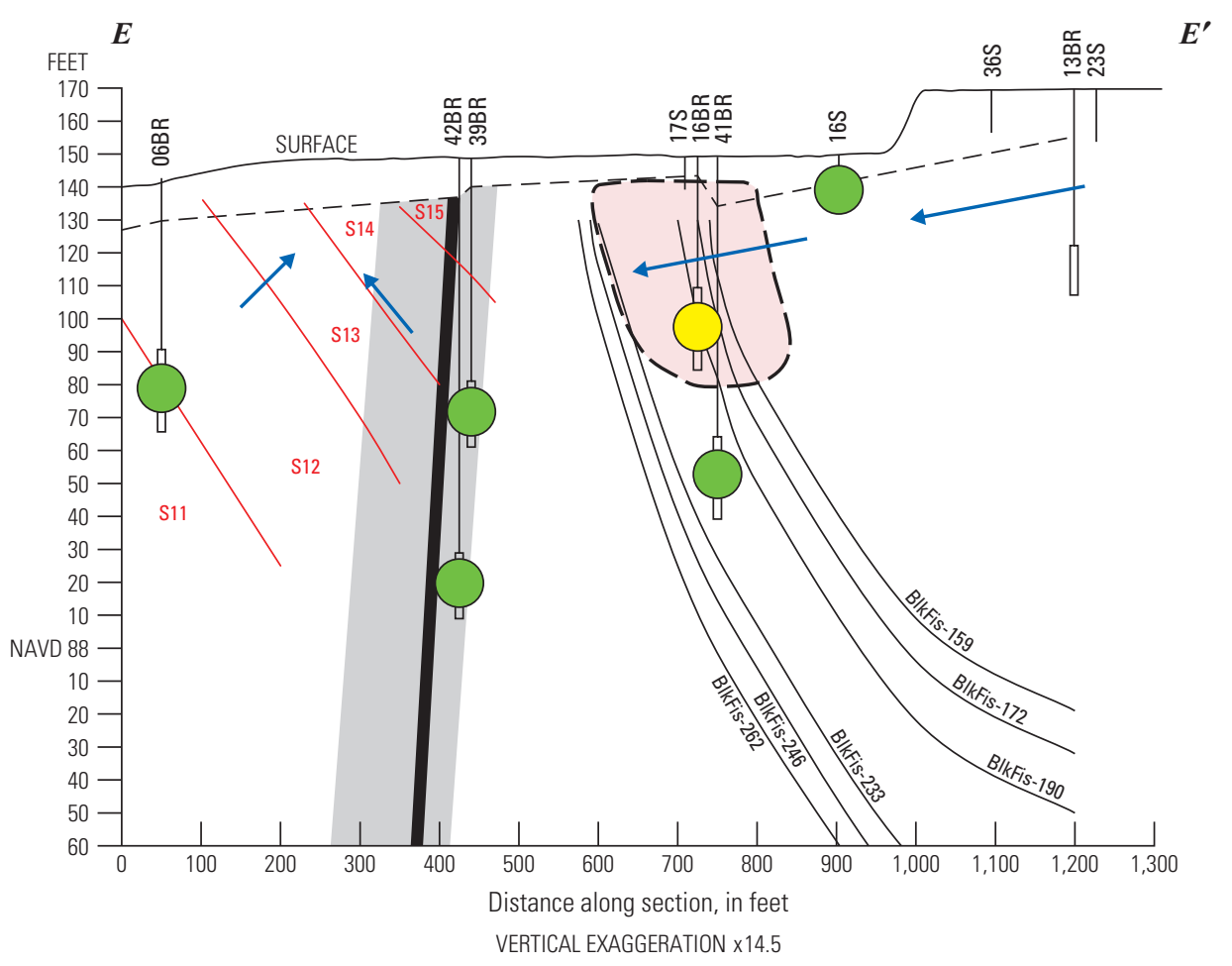

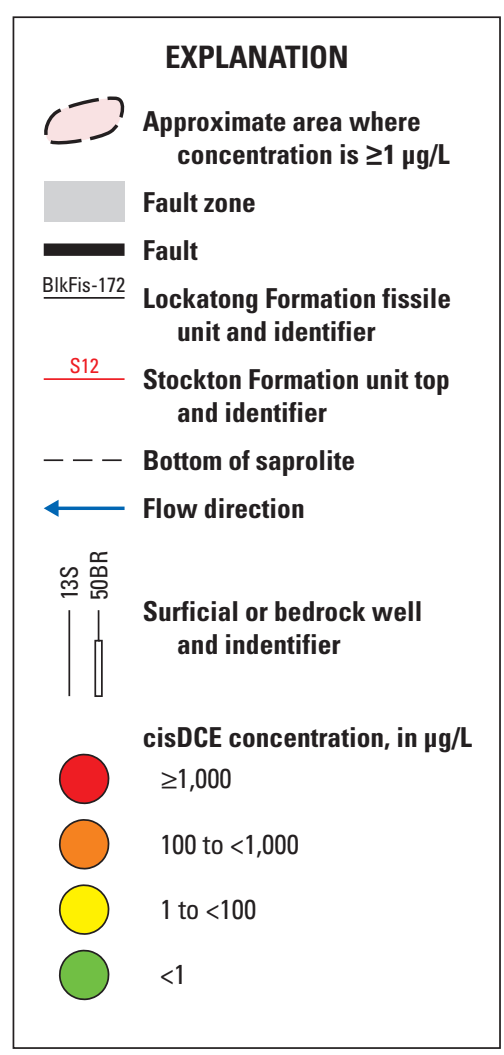

Figure 29. Vertical extent of cis-1,2-dichloroethene concentrations in wells along section $\mathrm{E}-\mathrm{E}$ ' in $A, 2014$ and B, 2015-17, former Naval Air Warfare Center, West Trenton, New Jersey. (cisDCE, cis-1,2-dichloroethene; mg/L, micrograms per liter) 


\section{A. 2014 cisDCE concentrations}

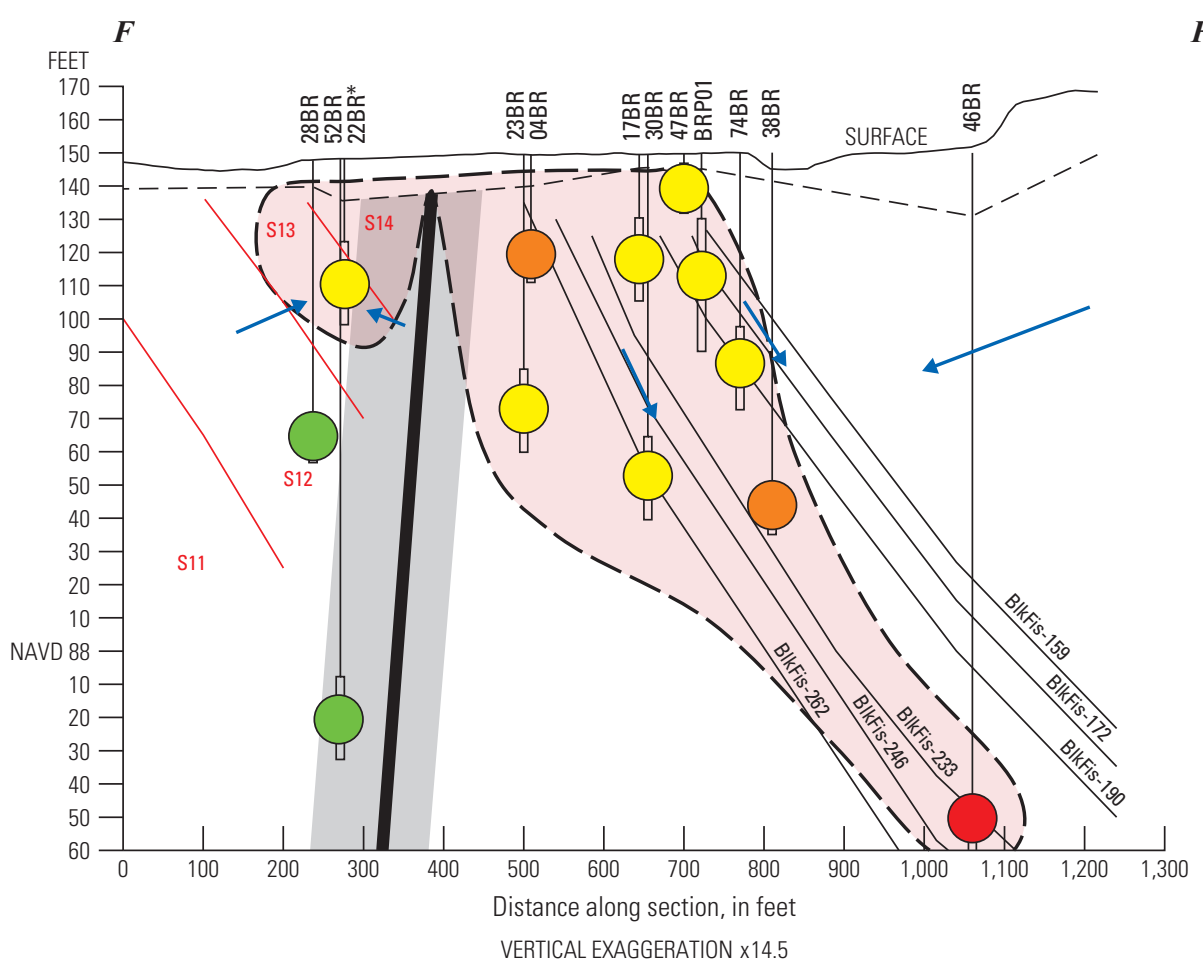

\section{B. 2015-17 cisDCE most recent concentrations}

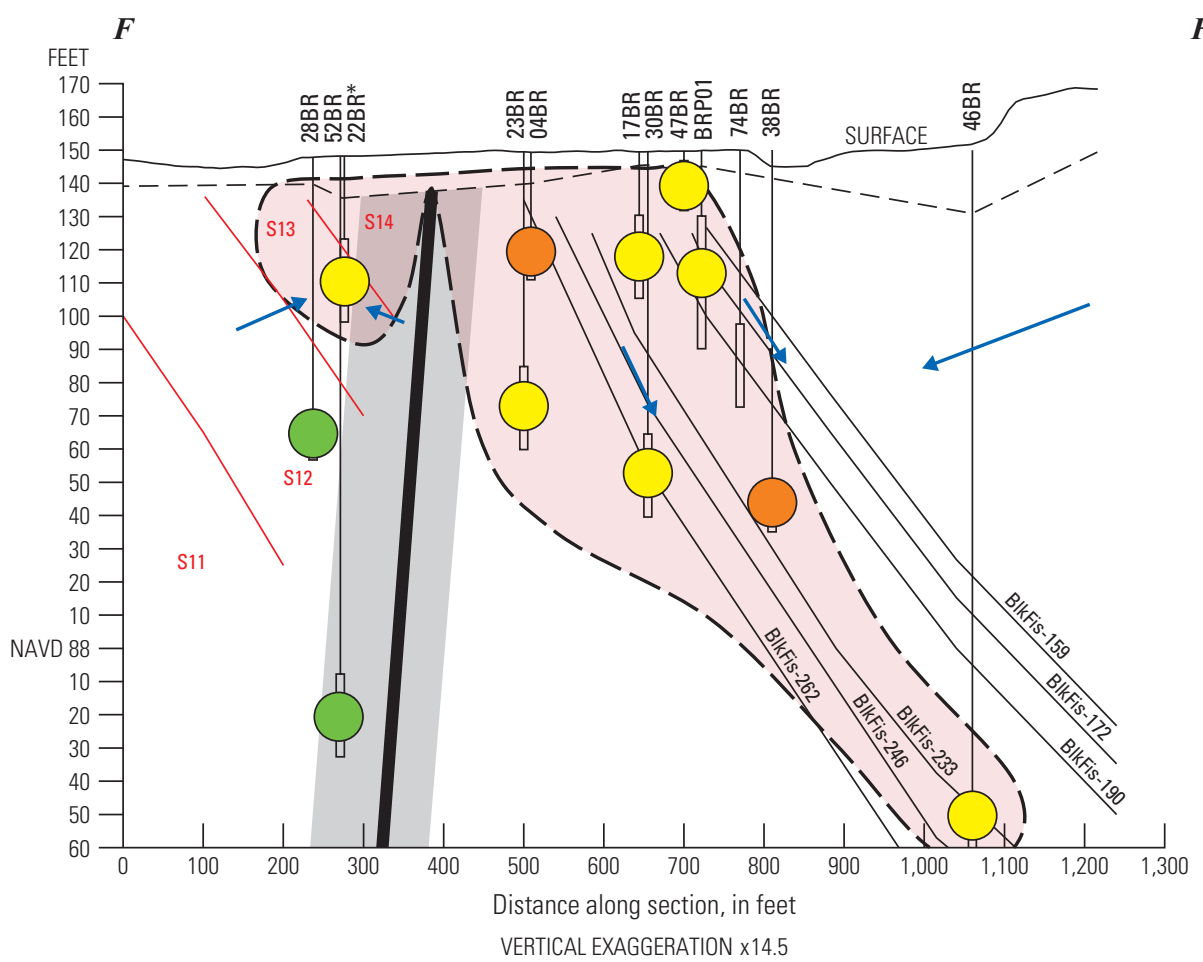

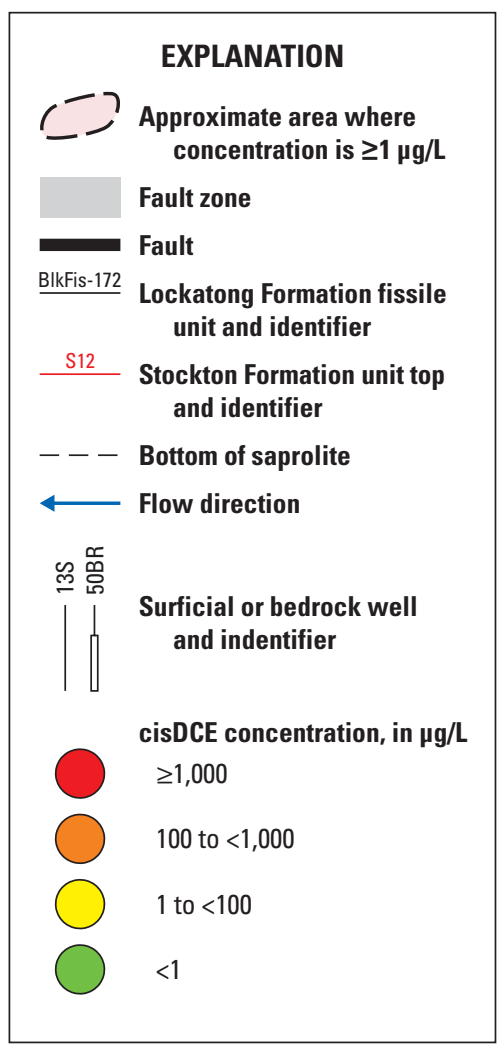

Figure 30. Vertical extent of cis-1,2-dichloroethene concentrations in wells along section $\mathrm{F}-\mathrm{F}^{\prime}$ in $A, 2014$ and B, 2015-17, former Naval Air Warfare Center, West Trenton, New Jersey. (cisDCE, cis-1,2-dichloroethene; mg/L, micrograms per liter) 


\section{A. 2014 cisDCE concentrations}

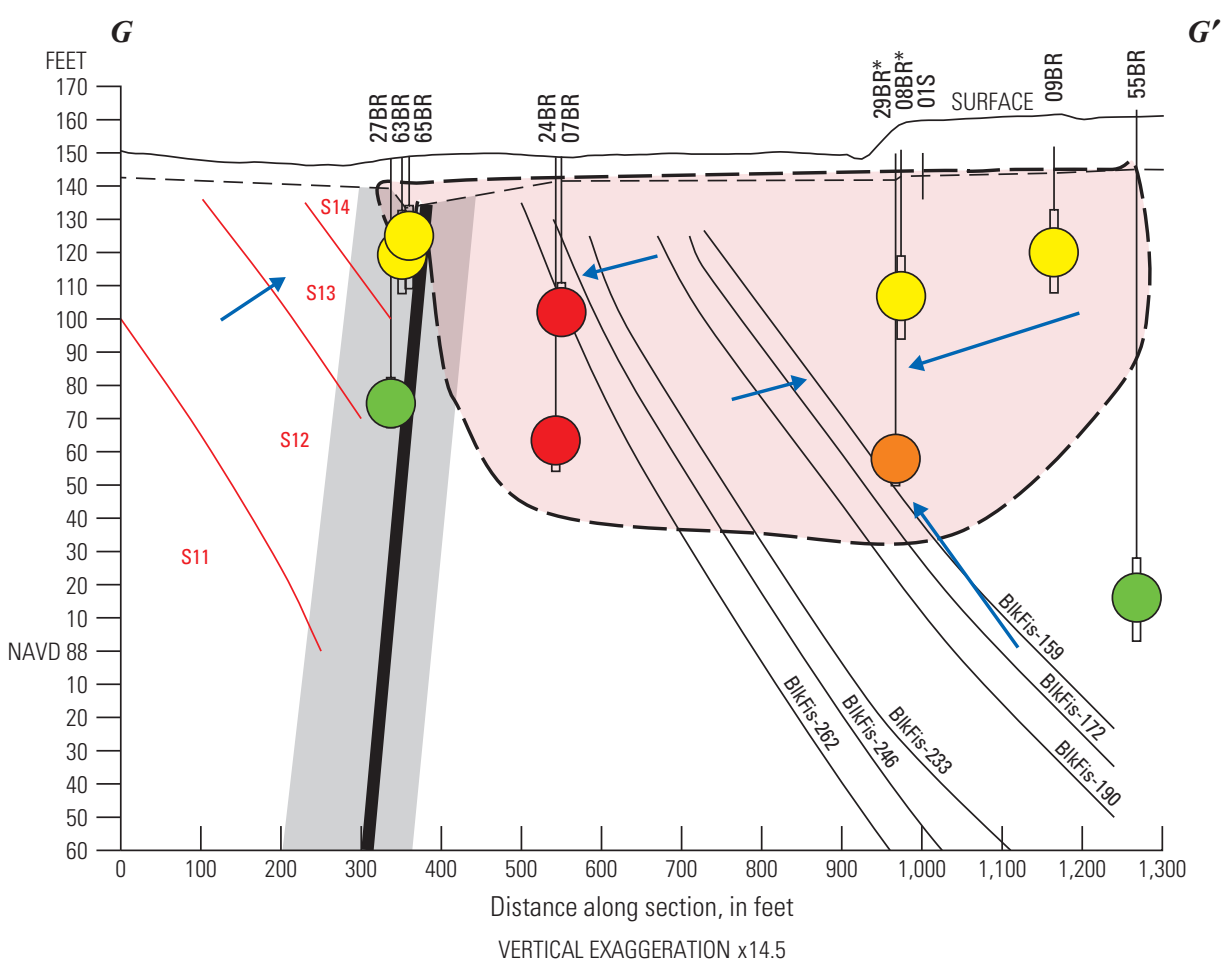

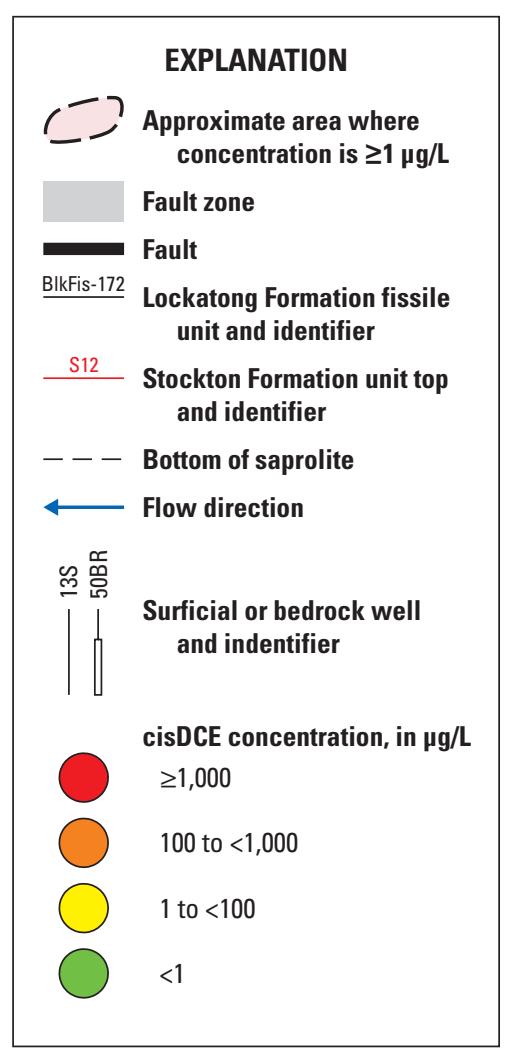

\section{B. 2015-17 cisDCE most recent concentrations}

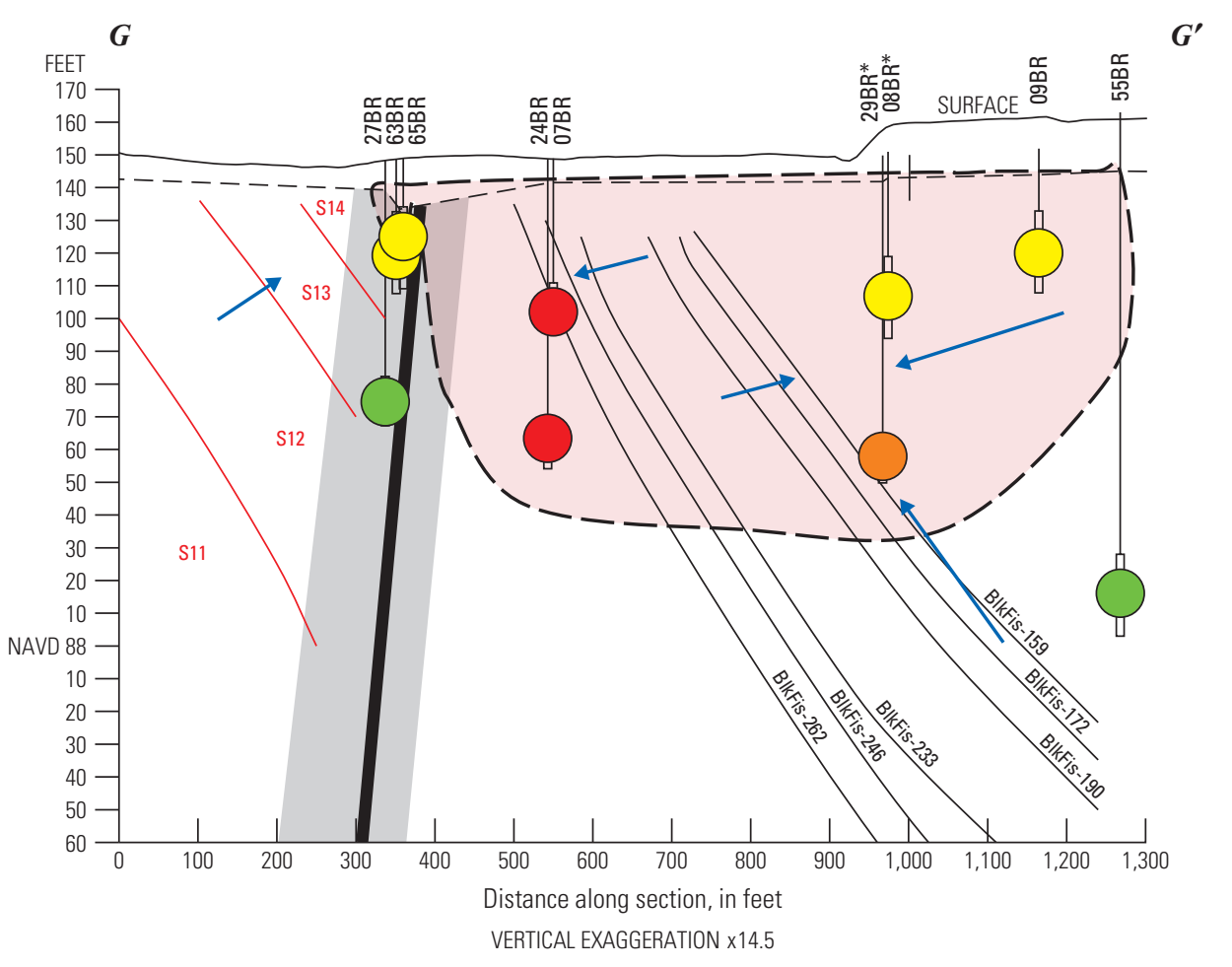

Figure 31. Vertical extent of cis-1,2-dichloroethene concentrations in wells along section $\mathrm{G}-\mathrm{G}$ ' in $A, 2014$ and $B$, 2015-17, former Naval Air Warfare Center, West Trenton, New Jersey. (cisDCE, cis-1,2-dichloroethene; mg/L, micrograms per liter) 


\section{A. 2014 cisDCE concentrations}

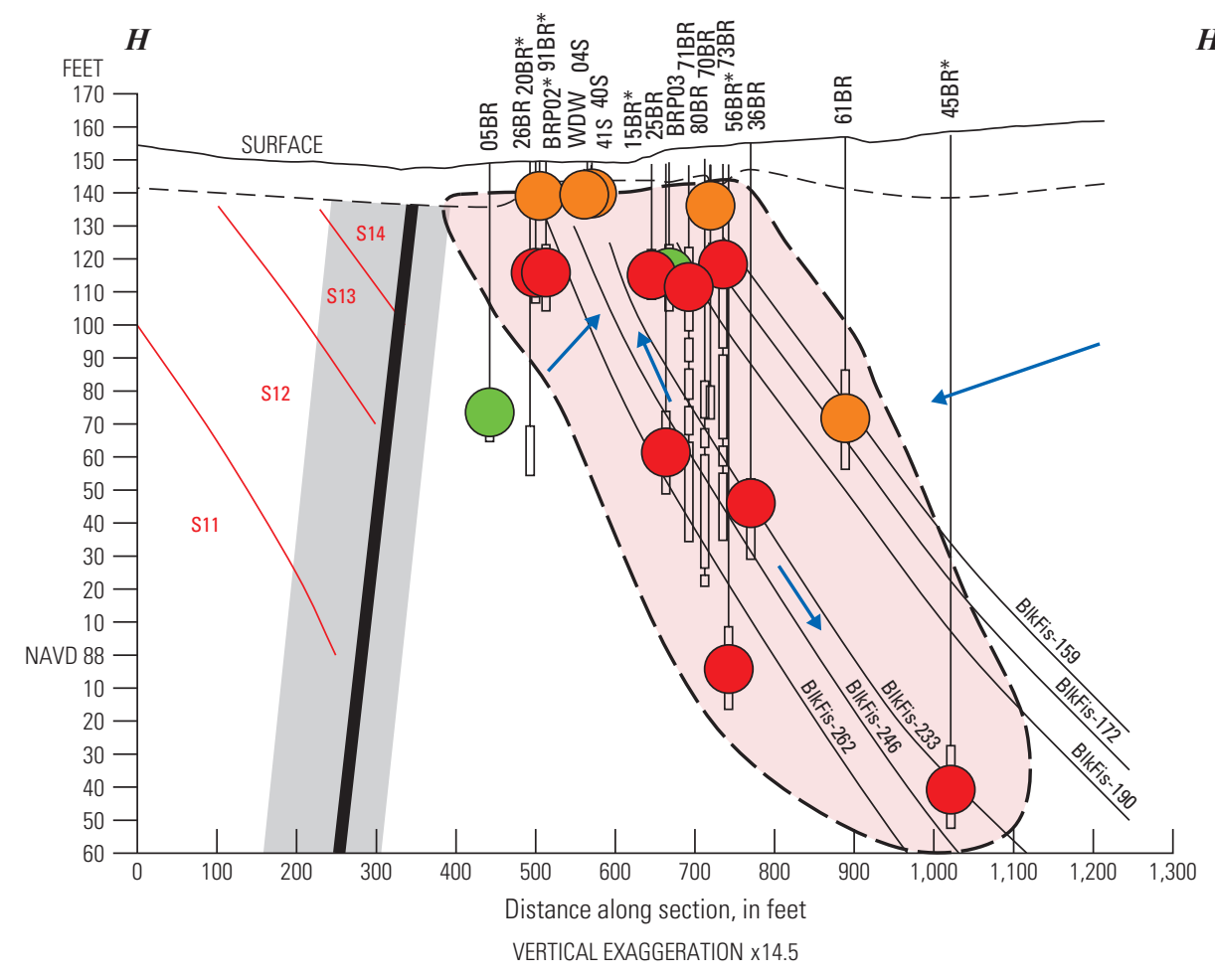

$\boldsymbol{H}^{\prime}$

\section{B. 2015-17 cisDCE most recent concentrations}

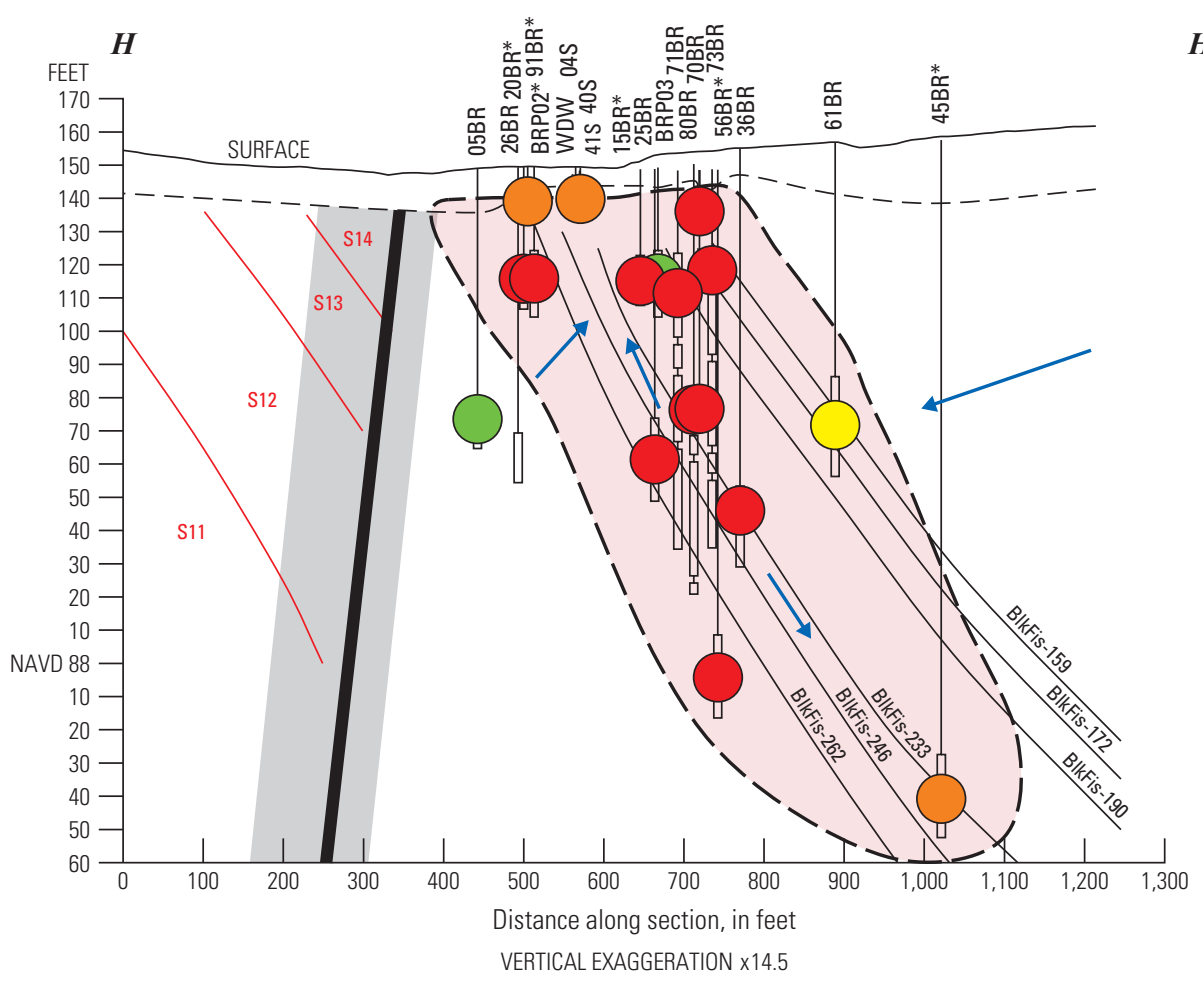

EXPLANATION
concentration is $\geq 1 \mu \mathrm{g} / \mathrm{L}$
Fault zone
Fault
Lockatong Formation fissile
unit and identifier
Stockton Formation unit top
and identifier

Figure 32. Vertical extent of cis-1,2-dichloroethene concentrations in wells along section $\mathrm{H}-\mathrm{H}$ ' in $A, 2014$ and $B, 2015-17$, former Naval Air Warfare Center, West Trenton, New Jersey. (cisDCE, cis-1,2-dichloroethene; mg/L, micrograms per liter) 


\section{A. 2014 cisDCE concentrations}

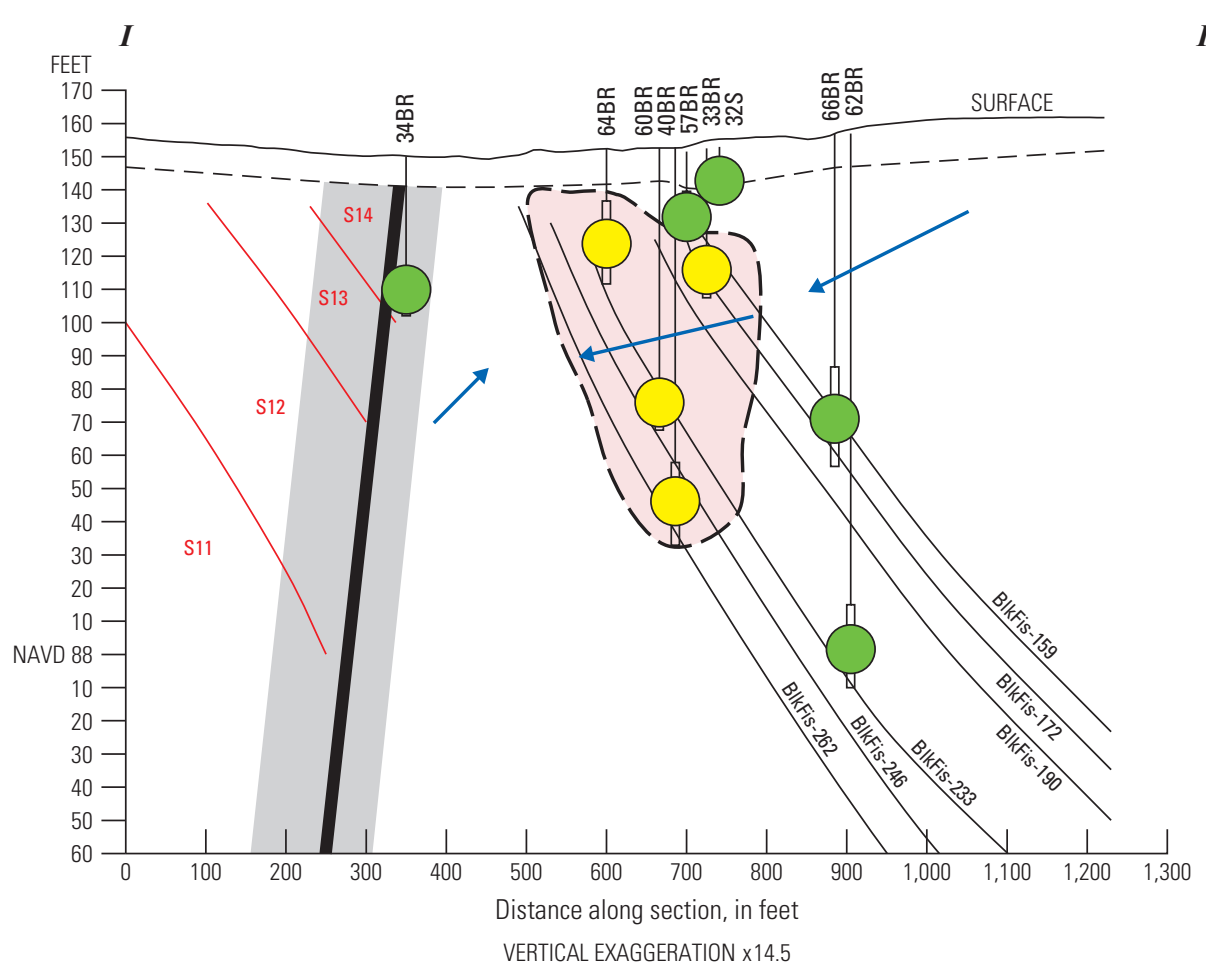

\section{B. 2015-17 cisDCE most recent concentrations}

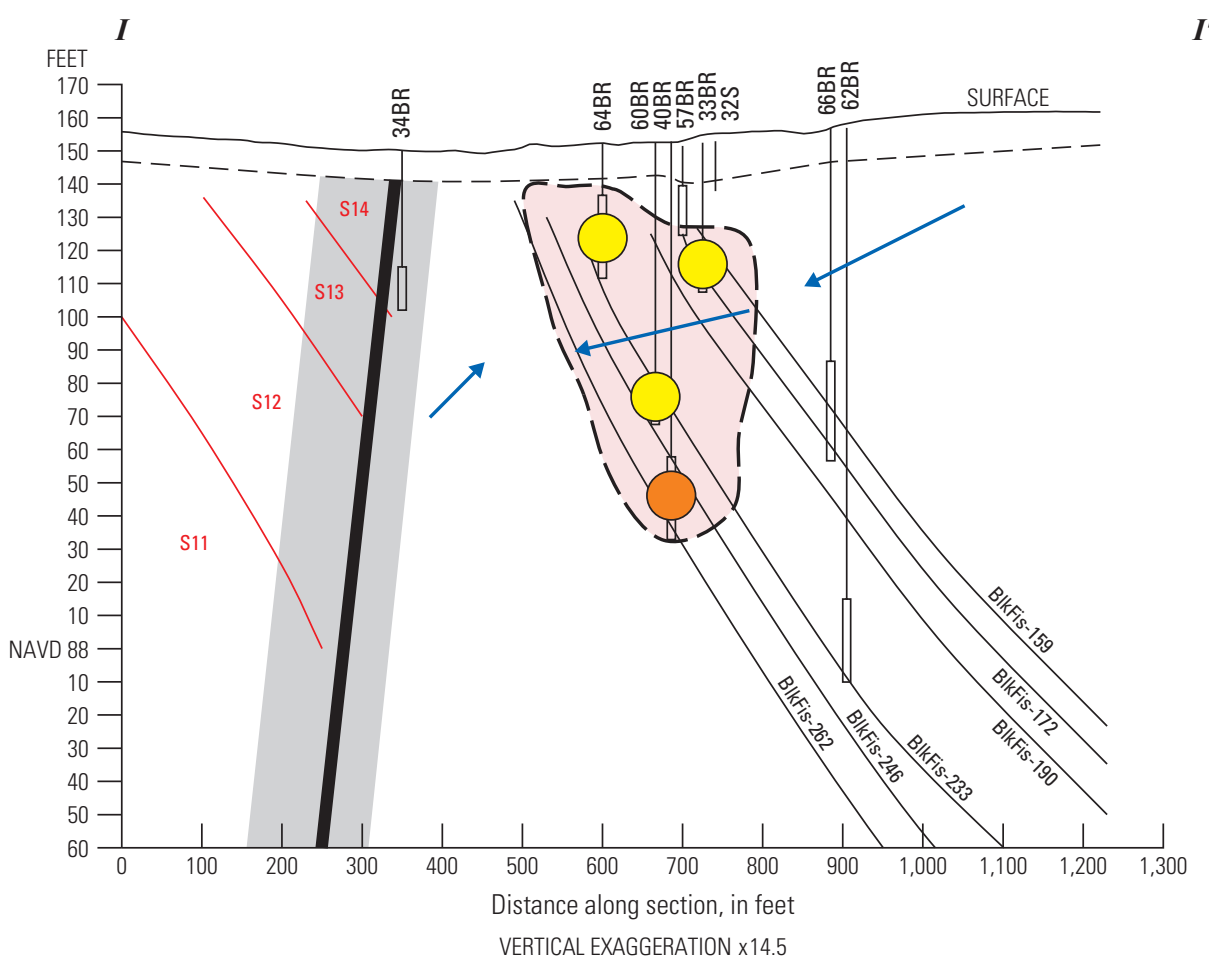

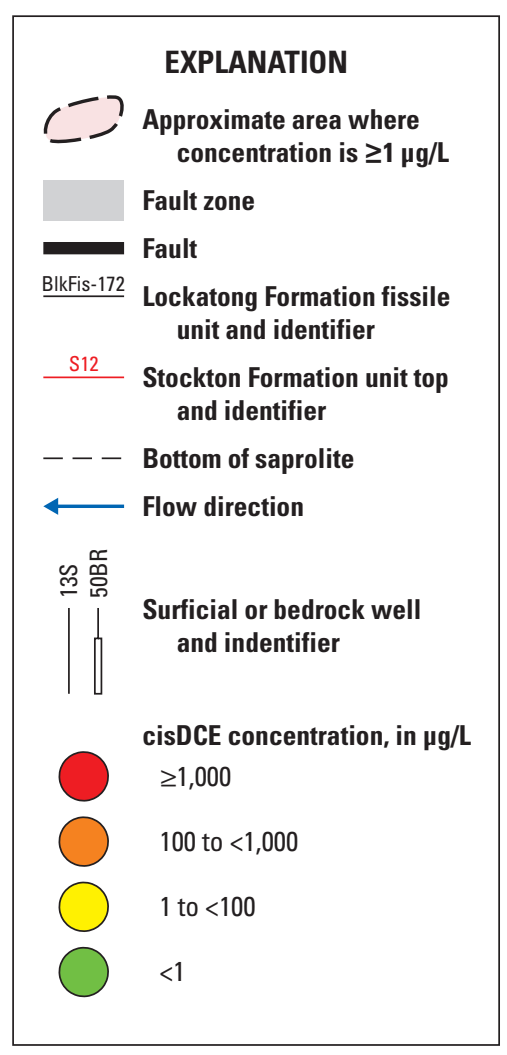

Figure 33. Vertical extent of cis-1,2-dichloroethene concentrations in wells along section I-I' in $A, 2014$ and $B, 2015-17$, former Naval Air Warfare Center, West Trenton, New Jersey. (cisDCE, cis-1,2-dichloroethene; mg/L, micrograms per liter) 
others, 1998). Because of this phenomenon, VC is often present at much lower concentrations than TCE and cisDCE in wells at NAWC.

\section{Site 3 Eastern Sections A-A' to D-D'}

No wells sampled along section A-A' were found to contain $\mathrm{VC}$ at concentrations $\geq 1 \mu \mathrm{g} / \mathrm{L}$ from 2014 to 2017 (fig. 34). On Section B-B', only well 02BR (60 ft deep) had VC concentrations $\geq 1 \mu \mathrm{g} / \mathrm{L}$ during $2014-17$ (fig. 35). VC concentrations in well 02BR were low, ranging from 1.3 to $1.8 \mu \mathrm{g} / \mathrm{L}$.

Along section $\mathrm{C}-\mathrm{C}^{\prime}$, the vertical distribution of $\mathrm{VC}$ in 2014 was estimated to extend from shallow groundwater to $200 \mathrm{ft}$ below land surface in well 54BR (fig. 36A). Wells 48BR $(1.2 \mu \mathrm{g} / \mathrm{L})$ and 54BR $(1.5 \mu \mathrm{g} / \mathrm{L})$ were the only wells with concentrations of $\mathrm{VC} \geq 1 \mu \mathrm{g} / \mathrm{L}$ in 2014. In 2017, VC concentrations in these two wells were $<1 \mu \mathrm{g} / \mathrm{L}$, so the vertical area of contamination was eliminated on figure 36B. No wells sampled on section D-D' were found to contain VC concentrations $\geq 1 \mu \mathrm{g} / \mathrm{L}$ during 2014-17 (fig. 37).

\section{Site 1 Western Sections E-E' to I-I'}

The vertical distribution of $\mathrm{VC}$ concentrations $\geq 1 \mu \mathrm{g} / \mathrm{L}$ in wells along section E-E' ranged from shallow groundwater to $110 \mathrm{ft}$ below land surface in well 41BR in 2014 (fig. 38A). The only wells with VC concentrations $\geq 1$ $\mu \mathrm{g} / \mathrm{L}$ were $16 \mathrm{BR}(2.0 \mu \mathrm{g} / \mathrm{L})$ and 41BR $(4.3 \mu \mathrm{g} / \mathrm{L})$. During 2015-17, the VC concentration in well 16BR stayed the same, whereas in well 41BR, the concentration decreased to $<1 \mu \mathrm{g} / \mathrm{L}$ (fig. 38B). Because of this decrease, the vertical extent of VC contamination was smaller for 2015-17.

The vertical distribution of VC concentrations in wells on section $\mathrm{F}-\mathrm{F}^{\prime}$ extended from shallow groundwater to $221 \mathrm{ft}$ below land surface in well 46BR in 2014 (fig. 39A). Seven wells along this section had VC concentrations $\geq 1$ $\mu \mathrm{g} / \mathrm{L}$ in 2014 . The two wells with the highest VC concentrations in 2014 were 38BR $(1,100 \mu \mathrm{g} / \mathrm{L})$ and 30BR $(150 \mu \mathrm{g} / \mathrm{L})$ (fig. 39A). During 2015-17, VC concentrations decreased in wells 38BR (to $670 \mu \mathrm{g} / \mathrm{L}$ ) and 30BR (to $6.3 \mu \mathrm{g} / \mathrm{L}$ ) (fig. 39B). These decreases along with a decrease in $\mathrm{VC}$ concentration in well 23BR (from 1.5 to $<1 \mu \mathrm{g} / \mathrm{L}$ ) account for the differences in the $\mathrm{VC}$ contamination extent in wells along this section over the 4-year period. Well 38BR and well 30BR were part of the 2005 bioaugmentation study in which bacteria and ESO were injected to stimulate degradation of TCE (Geosyntec Consultants, 2010).

Along section $\mathrm{G}-\mathrm{G}^{\prime}$, the vertical distribution of $\mathrm{VC}$ in 2014 was estimated to extend from shallow groundwater to $100 \mathrm{ft}$ below land surface in well 29BR (fig. 40A). Only three wells had VC concentrations $\geq 1 \mu \mathrm{g} / \mathrm{L}$ in 2014-wells 07BR $(930 \mu \mathrm{g} / \mathrm{L}), 24 \mathrm{BR}(330 \mu \mathrm{g} / \mathrm{L})$, and 29BR $(7.3 \mu \mathrm{g} / \mathrm{L})$. During 2015-17, VC concentrations remained about the same in 07BR and 29BR but increased in 24BR (to $990 \mu \mathrm{g} / \mathrm{L}$ ) (fig. 40B). VC concentrations increased from $<1 \mu \mathrm{g} / \mathrm{L}$ to 3.4 $\mu \mathrm{g} / \mathrm{L}$ in well 65BR and from $<1 \mu \mathrm{g} / \mathrm{L}$ to $2.3 \mu \mathrm{g} / \mathrm{L}$ in well 09BR from 2014 to 2015-17. These increases are the cause for the expanded VC contamination extent on Section $\mathrm{G}-\mathrm{G}$ ' for 2015-17.

Like the TCE and cisDCE vertical distributions, VC contamination was present in wells along section $\mathrm{H}-\mathrm{H}$ ' from shallow groundwater to $210 \mathrm{ft}$ below land surface in well 45BR in 2014 (fig. 41A). Wells 36BR (15,000 $\mu \mathrm{g} / \mathrm{L})$ and BRP02 (2,900 $\mu \mathrm{g} / \mathrm{L}$ ) had the highest VC concentrations of the wells along this section and of all wells at the NAWC site in 2014. During 2015-17, VC concentrations remained high in wells 36BR $(4,500 \mu \mathrm{g} / \mathrm{L})$ and in BRP02 (2,400 $\mu \mathrm{g} / \mathrm{L})$ (fig. 41B). The main reason for the decrease in the vertical area of $\mathrm{VC}$ contamination between the two periods is that the $\mathrm{VC}$ concentration in well $61 \mathrm{BR}$ decreased from $9.1 \mu \mathrm{g} / \mathrm{L}$ in 2014 to $<1 \mu \mathrm{g} / \mathrm{L}$ in 2016 (fig. 41B).

Well 36BR was the injection well for the 2008 bioaugmentation project during which it received additional bacteria and ESO as a food source to stimulate anaerobic biodegradation of TCE (Shapiro and others, 2018). Most of the TCE has been degraded in this well, but cisDCE and VC concentrations have remained high owing to the stall in degradation of cisDCE to VC and VC to ethene. The decrease in VC concentration in 36BR from 2014 to 2017 may indicate that the ESO that was helping to stimulate biodegradation had finally been used up. Overall, these results confirmed that biodegradation is ongoing in the fractured rock aquifer underlying NAWC.

No VC concentrations $\geq 1 \mu \mathrm{g} / \mathrm{L}$ were present in samples from wells along section I-I' from 2014 to 2017 (fig. 42). This confirms that no VC is leaving the NAWC site via groundwater flow to the south or west.

\section{Variation in VOC Contamination over Time}

The variation in VOC concentrations over time in wells at the former NAWC site was evaluated by plotting graphs of TCE, cisDCE, and VC over time. Trends were not determined by statistical tests. The upward and downward trends discussed in this section were determined by visual interpretation of the plots of concentration versus time in figures 43 to 51. VOC data from 1990 through 2017 were included in the graphs to give a better picture of the way VOC concentrations have changed over the entire project timeline. The results are presented grouped by wells along each line of section starting on the eastern side of the NAWC site and moving towards the western side of the site. The data used to make these plots are presented in Appendix 2. All graphs have the same logarithmic concentration scale on the Y-axis and the same time scale on the $\mathrm{X}$-axis to allow for easy comparison of the VOC contamination levels between wells over time.

\section{Site 3 Eastern Sections A-A' to D-D'}

TCE, cisDCE, and VC concentrations in wells 11BR, $13 \mathrm{~S}$, and $14 \mathrm{~S}$ along section $\mathrm{A}-\mathrm{A}^{\prime}$ all had stable or downward trends over time, and concentrations in the three wells had 


\section{A. 2014 VC concentrations}

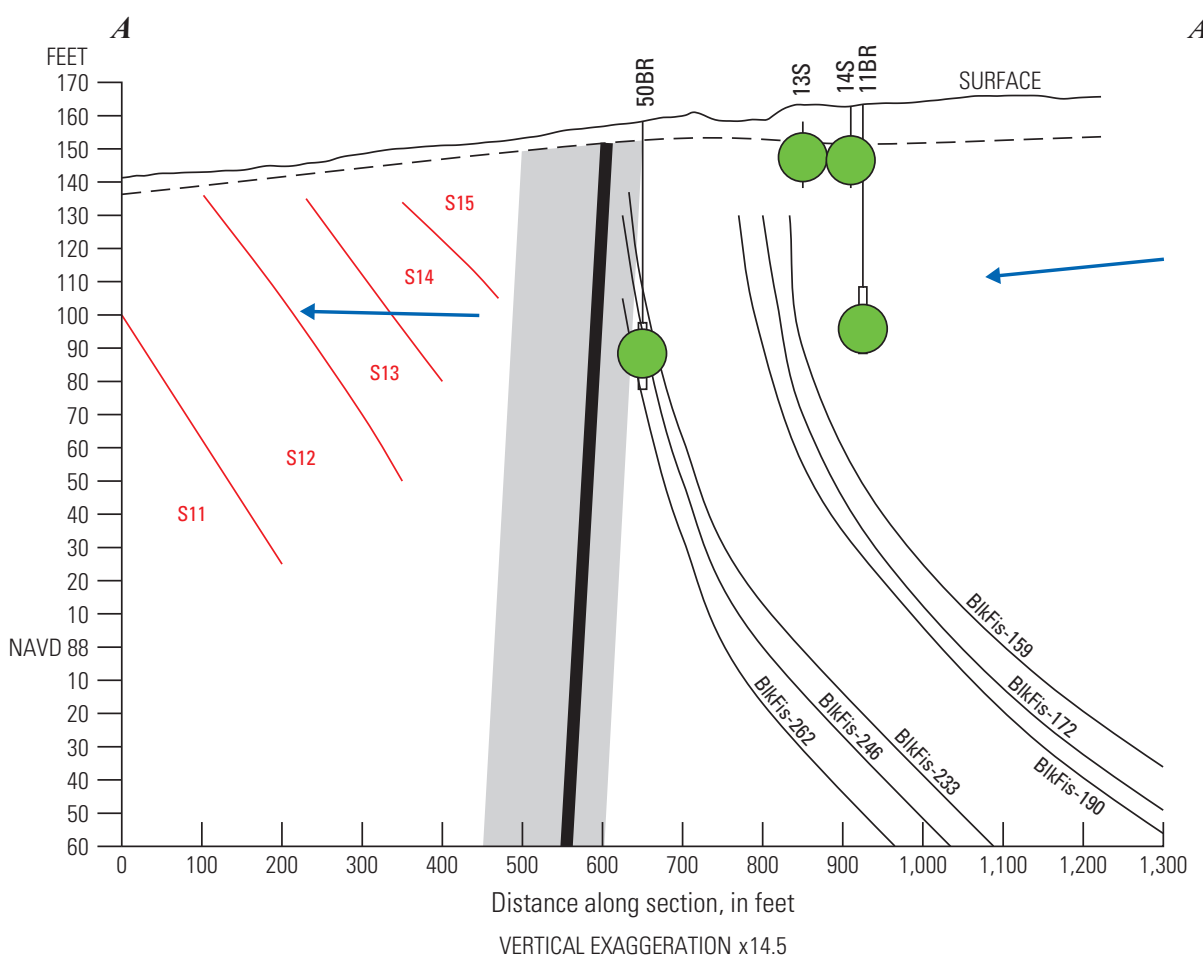

\section{B. 2015-17 VC most recent concentrations}

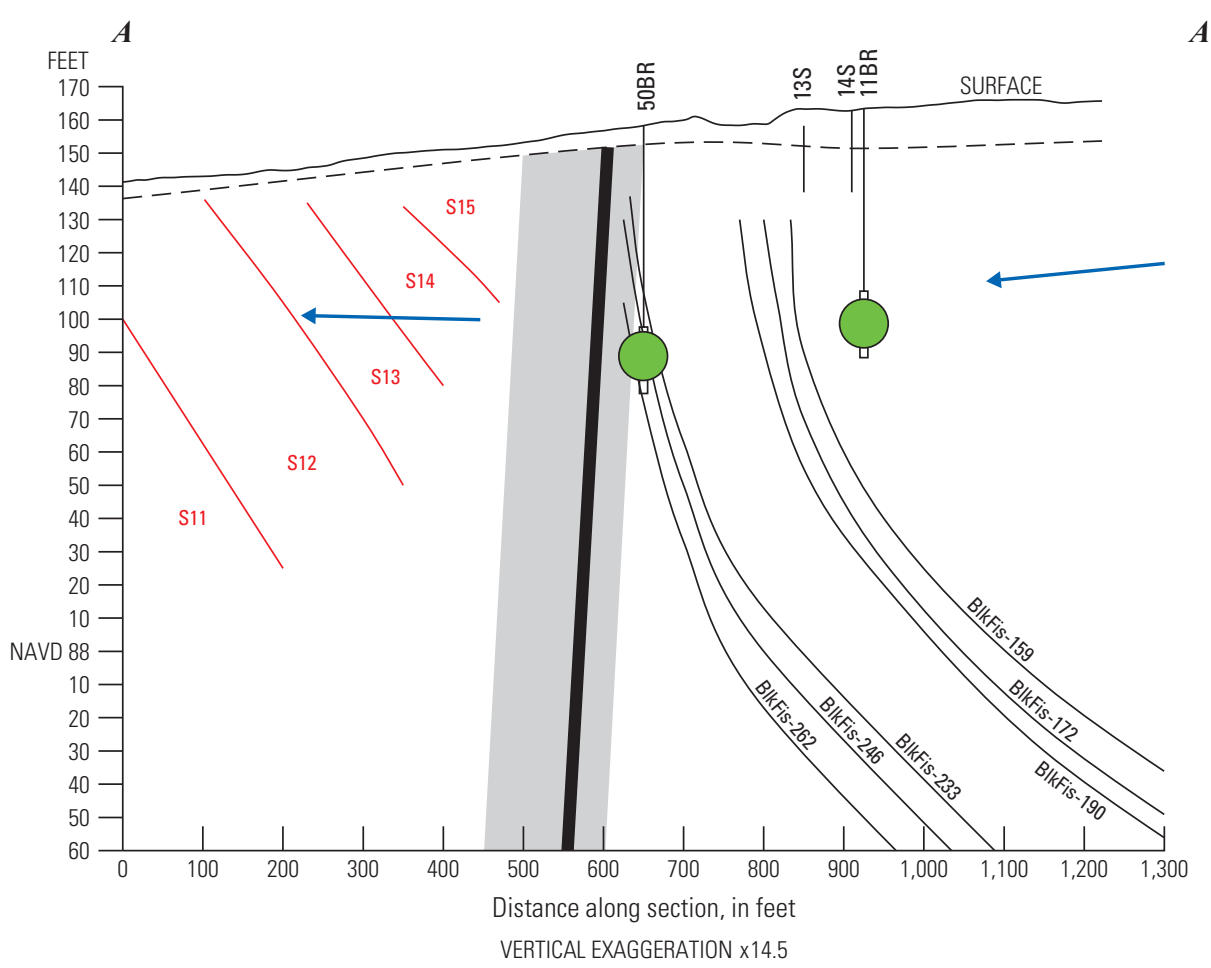

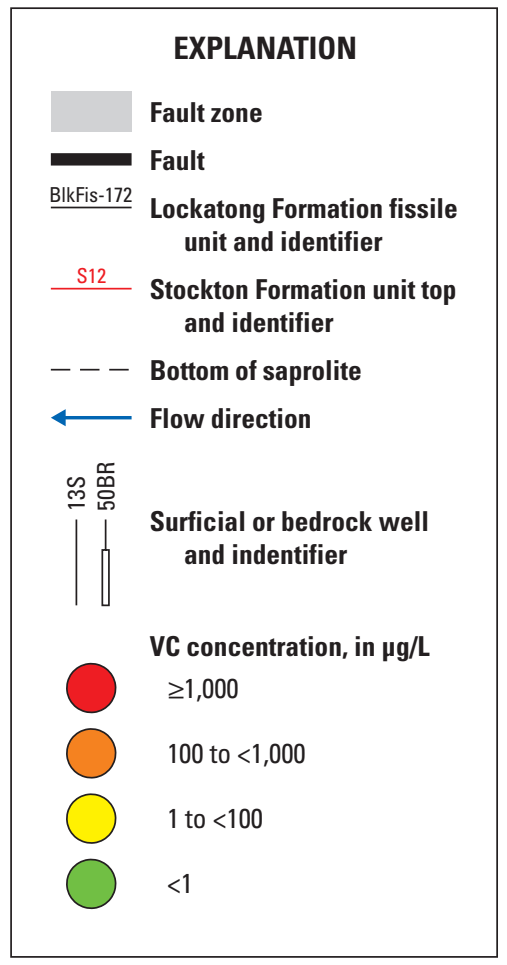

Figure 34. Vertical extent of vinyl chloride concentrations in wells along section $A-A^{\prime}$ in $A, 2014$ and $B, 2015-17$, former Naval Air Warfare Center, West Trenton, New Jersey. (VC, vinyl chloride; mg/L, micrograms per liter) 


\section{A. 2014 VC concentrations}

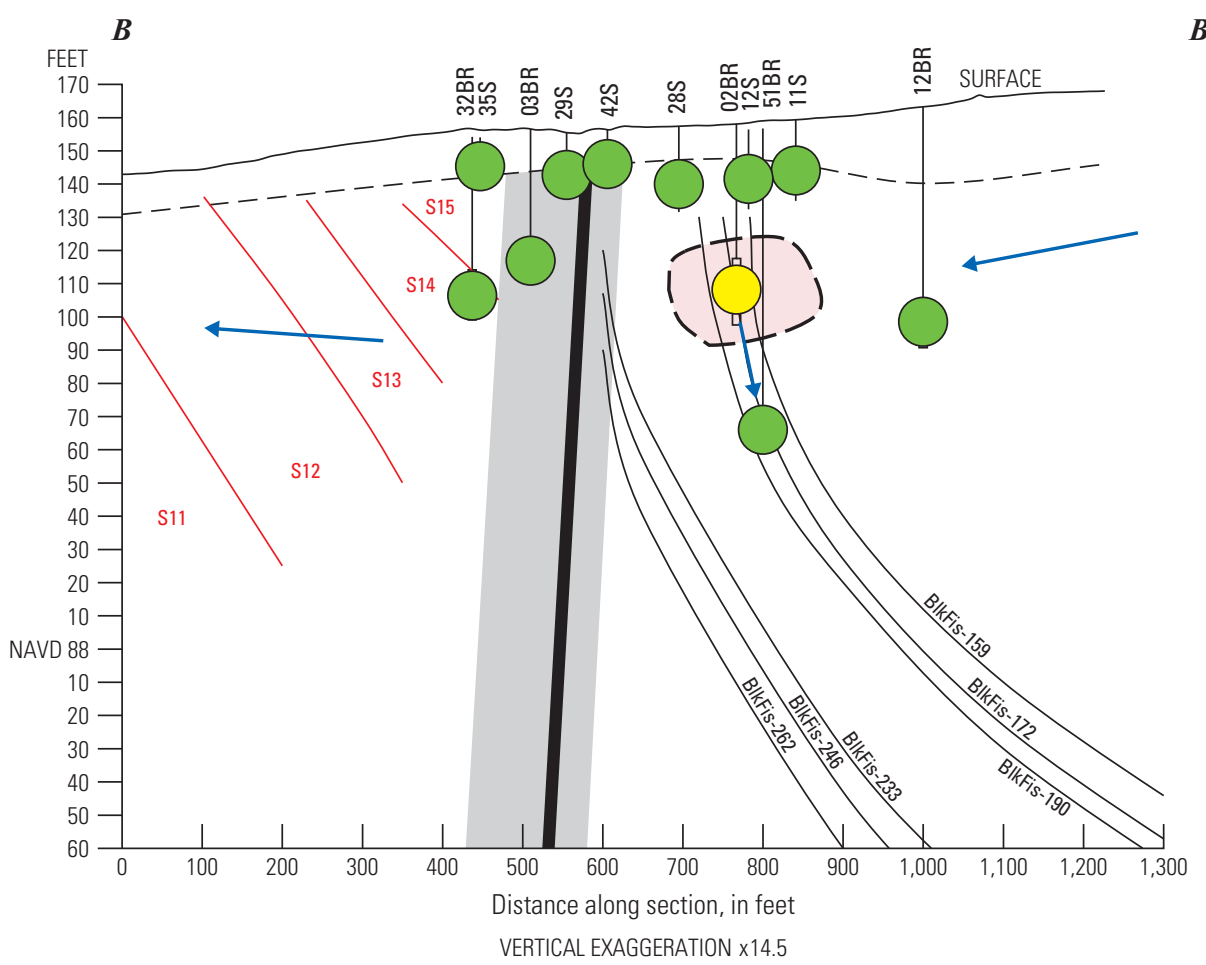

\section{B. 2015-17 VC most recent concentrations}

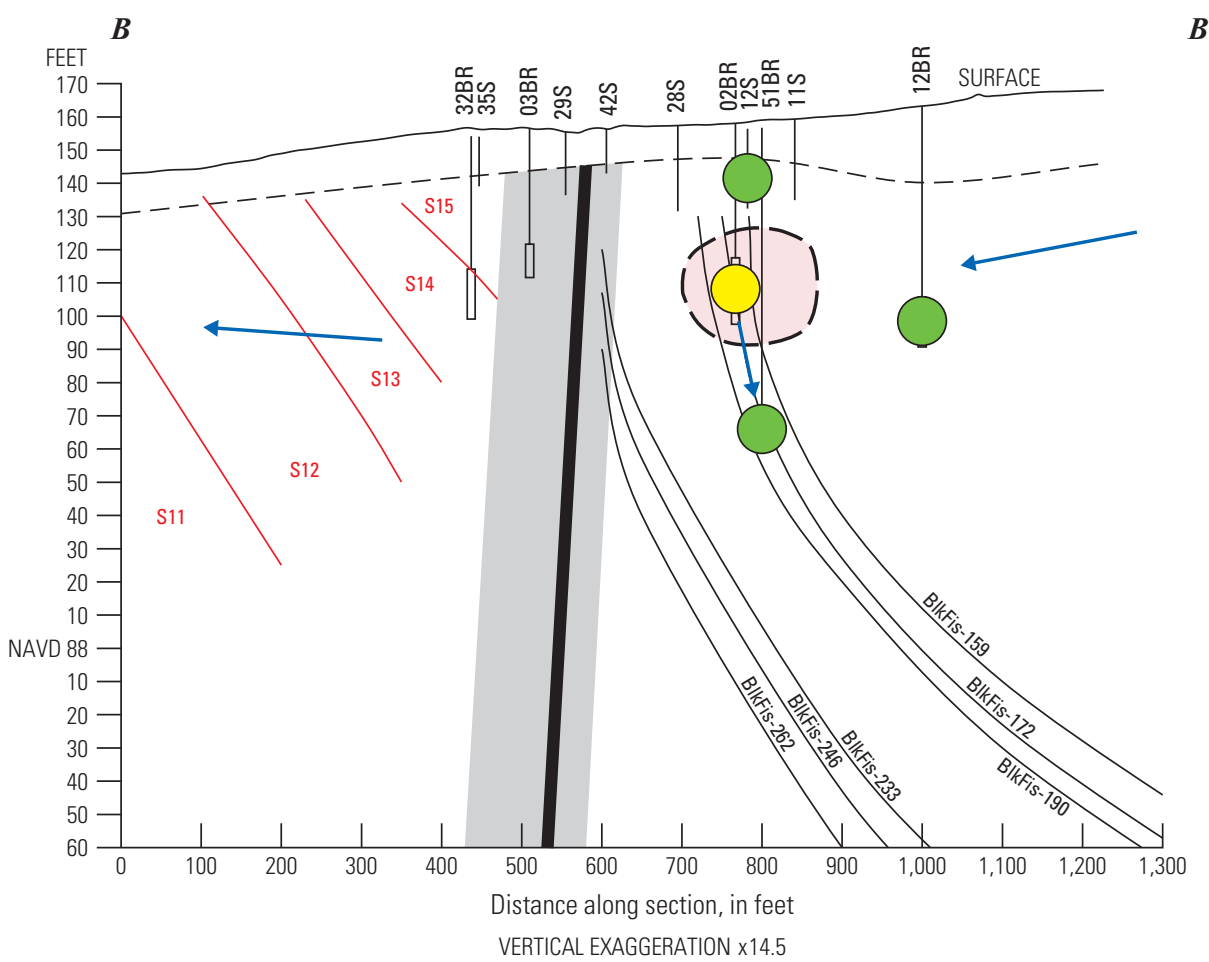

Approximate area where
concentration is $\geq 1 \mu \mathrm{g} / \mathrm{L}$
Fault zone
Fault
Lockatong Formation fissile
unit and identifier
Stockton Formation unit top
and identifier

Figure 35. Vertical extent of vinyl chloride concentrations in wells along section B-B' in $A, 2014$ and $B, 2015-17$, former Naval Air Warfare Center, West Trenton, New Jersey. (VC, vinyl chloride; mg/L, micrograms per liter) 


\section{A. 2014 VC concentrations}

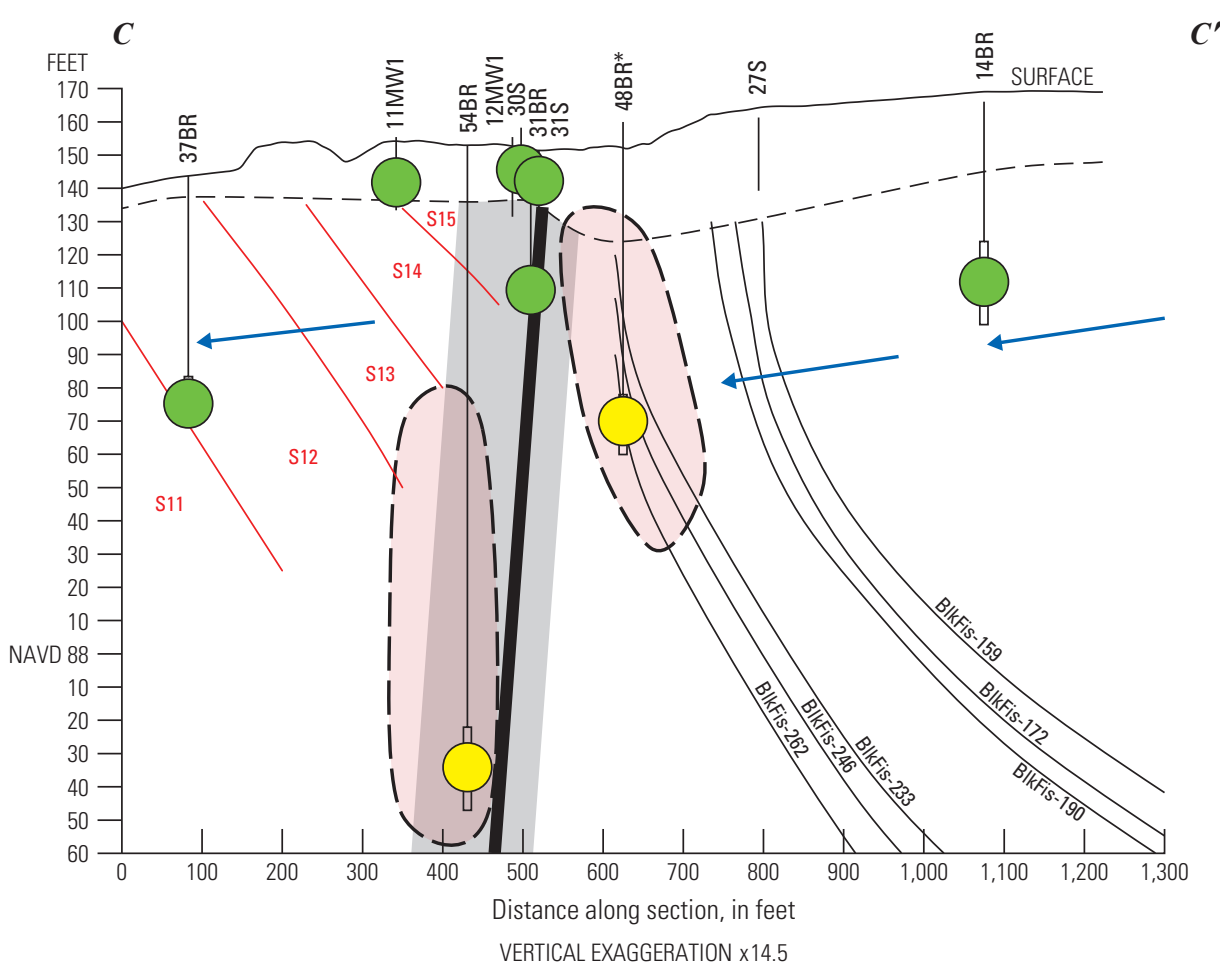

\section{B. 2015-17 VC most recent concentrations}

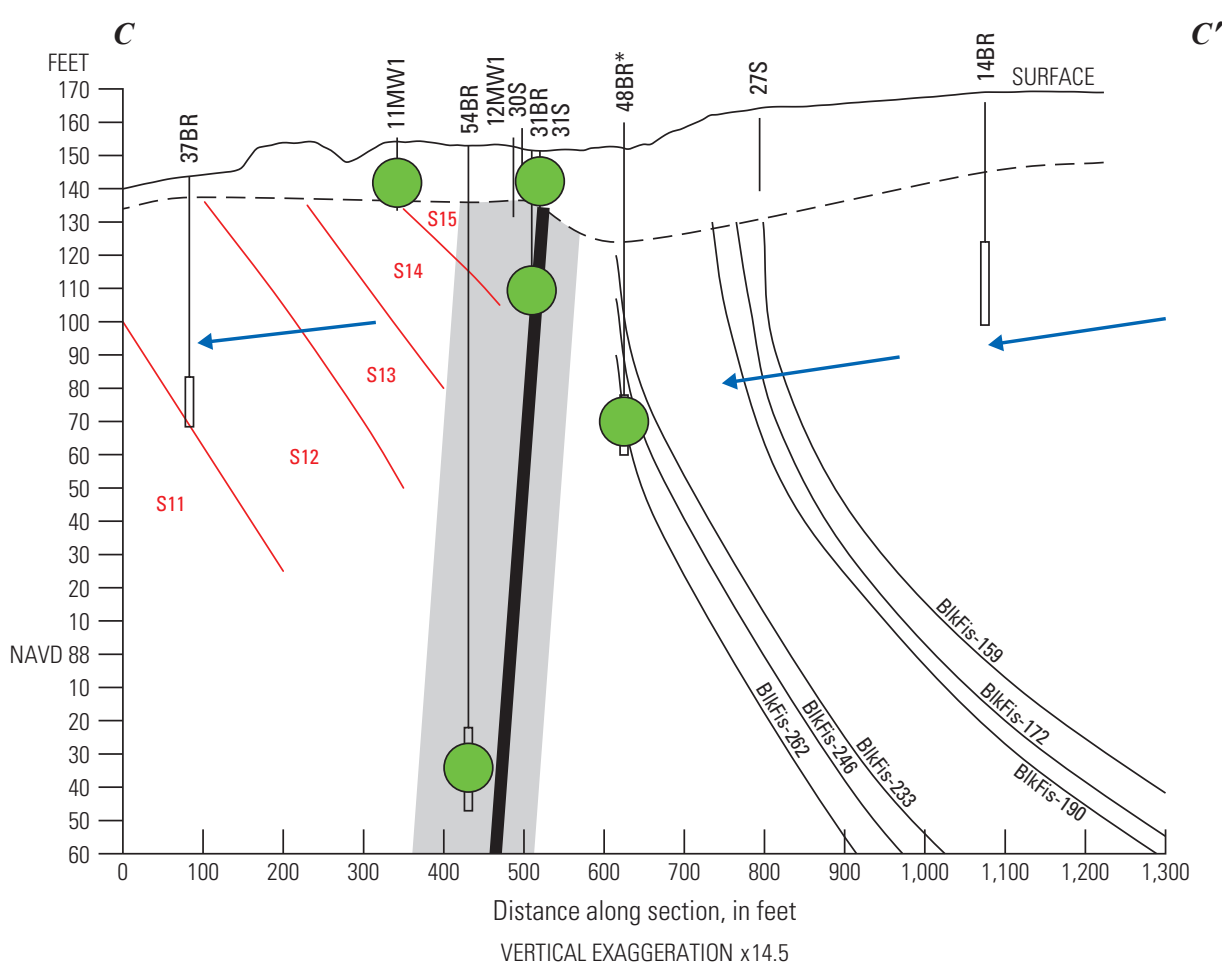

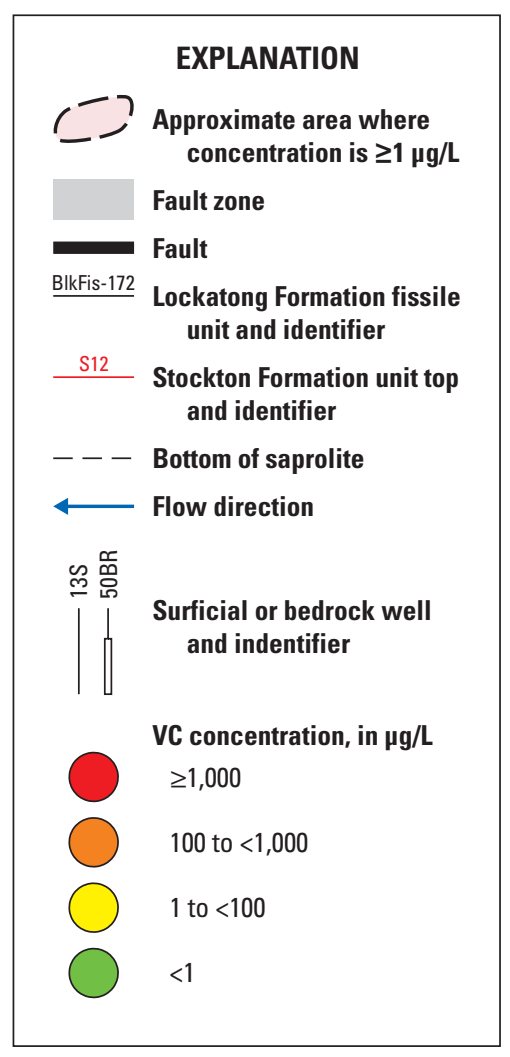

Figure 36. Vertical extent of vinyl chloride concentrations in wells along section $C-C^{\prime}$ in $A, 2014$ and $B, 2015-17$, former Naval Air Warfare Center, West Trenton, New Jersey. (VC, vinyl chloride; mg/L, micrograms per liter) 


\section{A. 2014 VC concentrations}

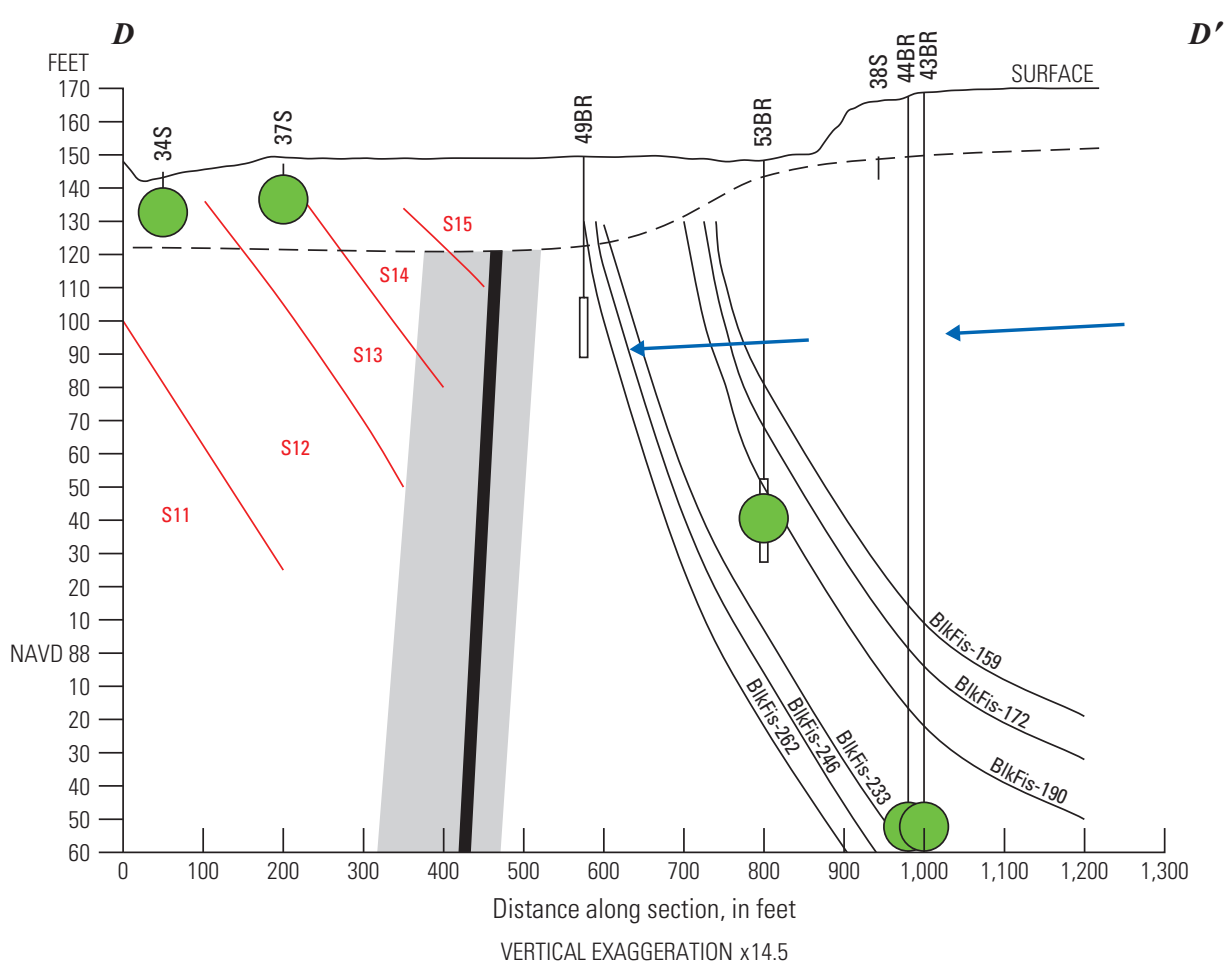

\section{B. 2015-17 VC most recent concentrations}

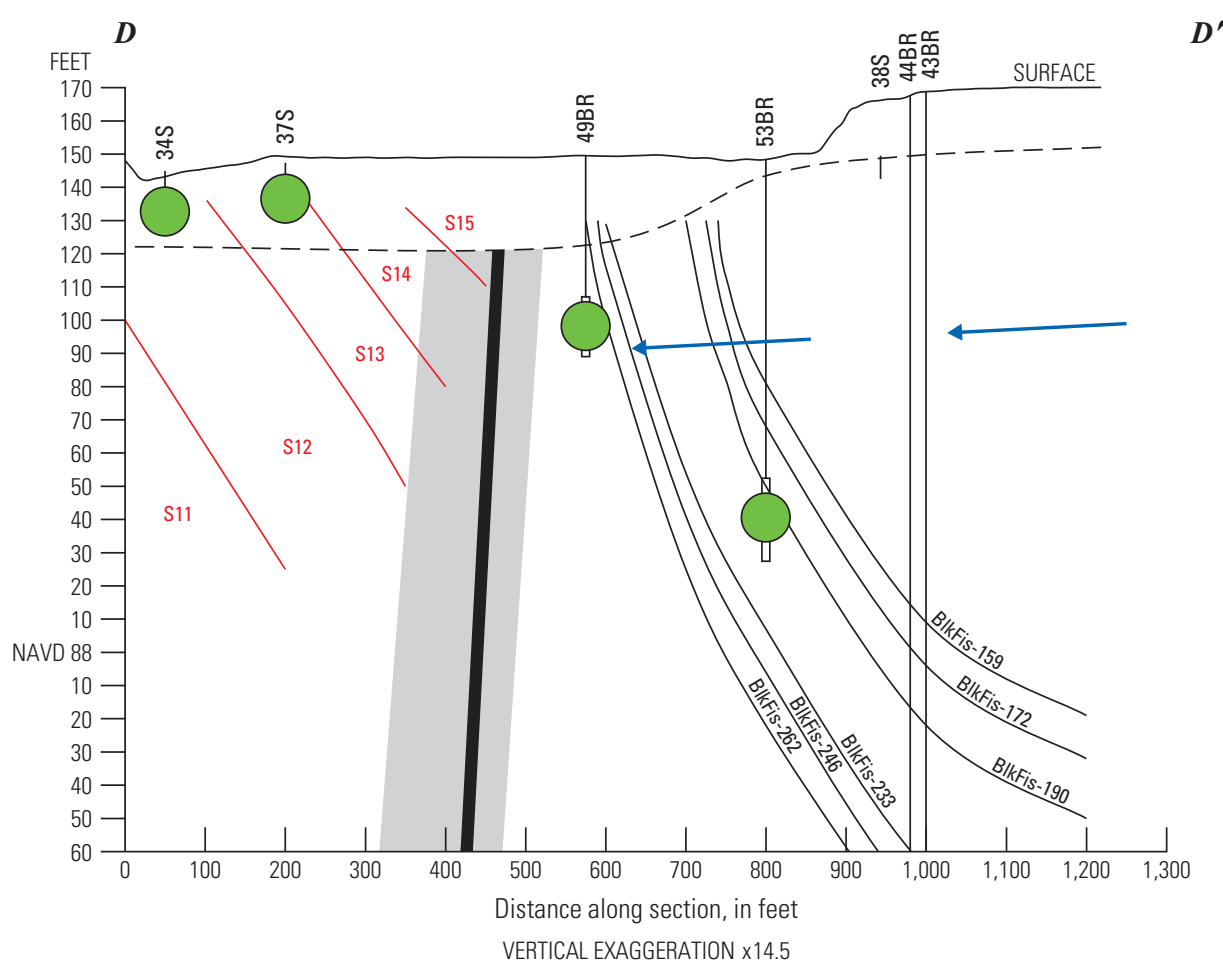

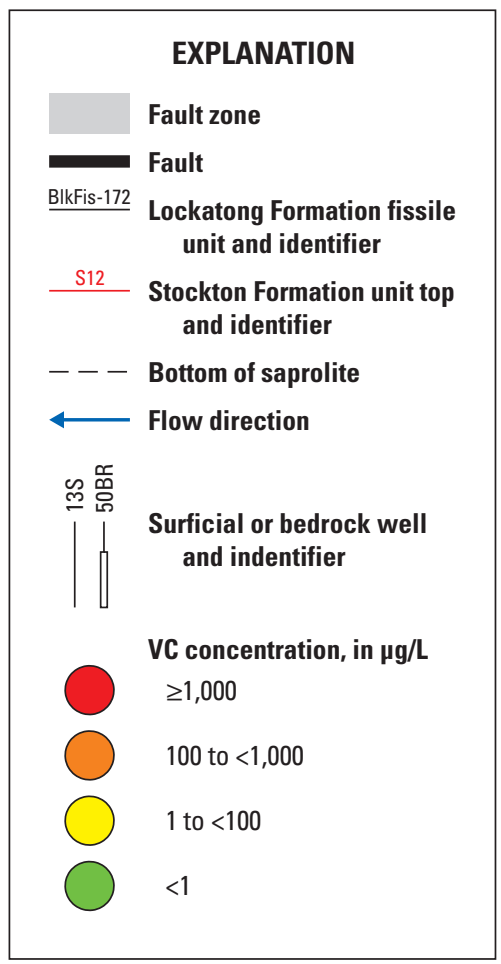

Figure 37. Vertical extent of vinyl chloride concentrations in wells along section D-D' in $A, 2014$ and B, 2015-17, former Naval Air Warfare Center, West Trenton, New Jersey. (VC, vinyl chloride; mg/L, micrograms per liter) 


\section{A. 2014 VC concentrations}

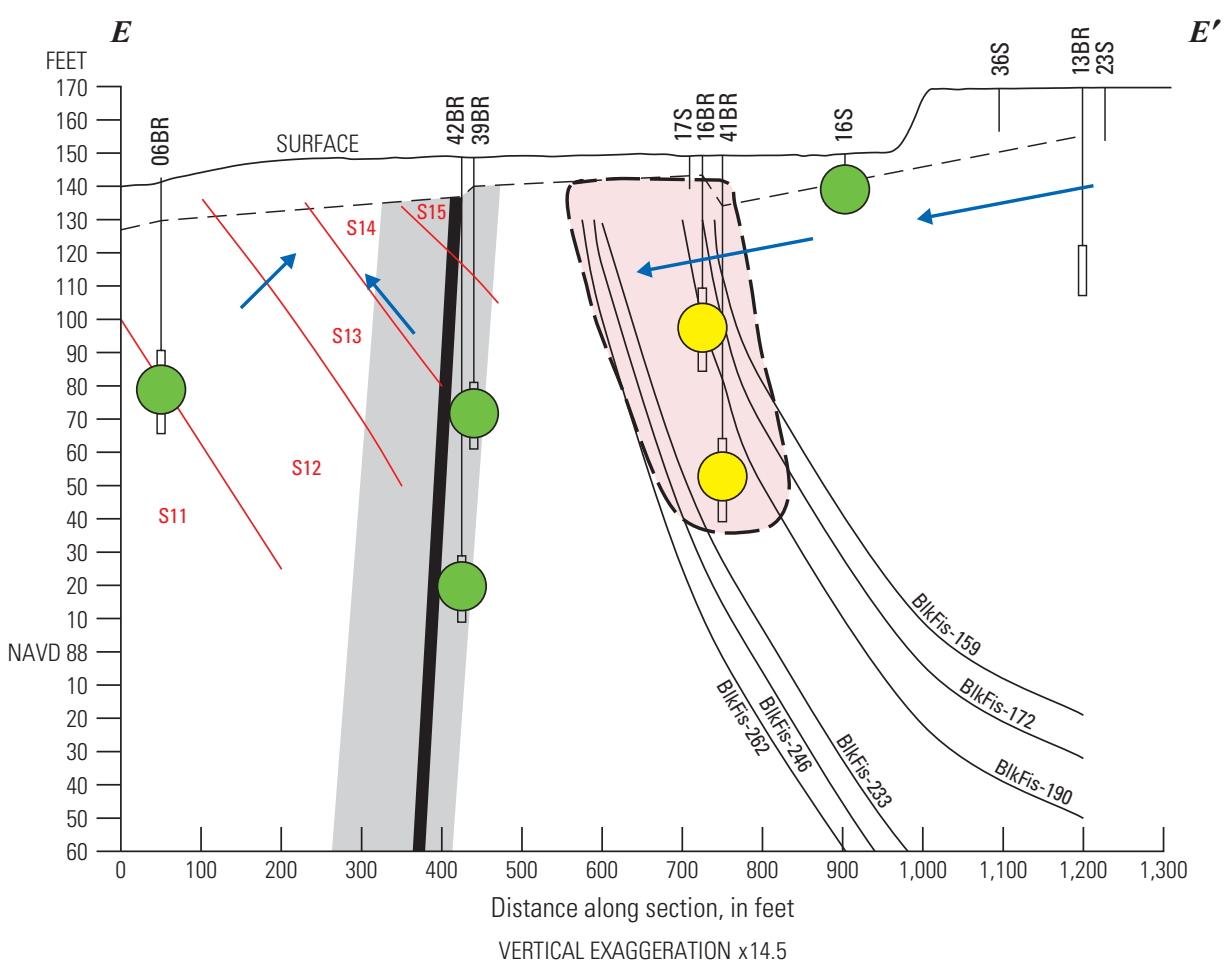

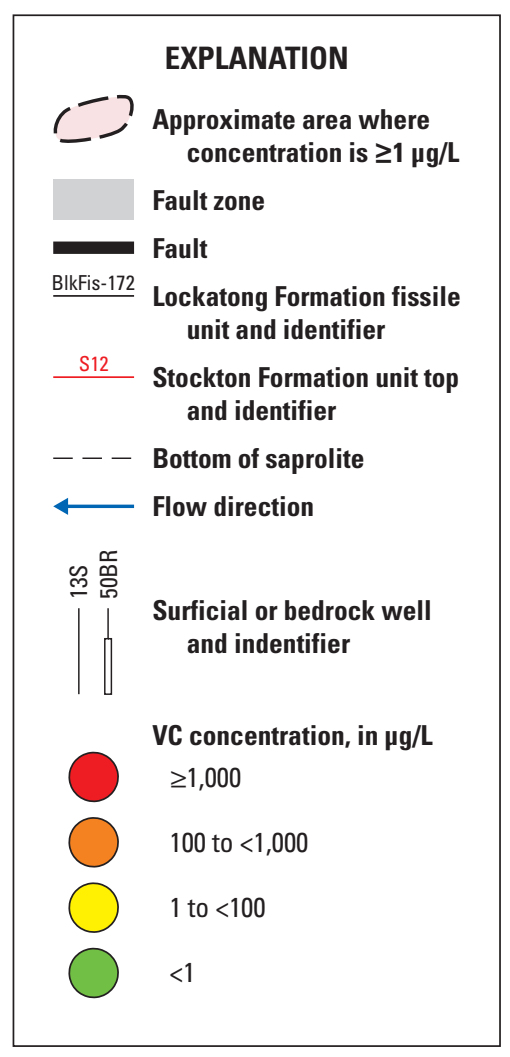

\section{B. 2015-17 VC most recent concentrations}

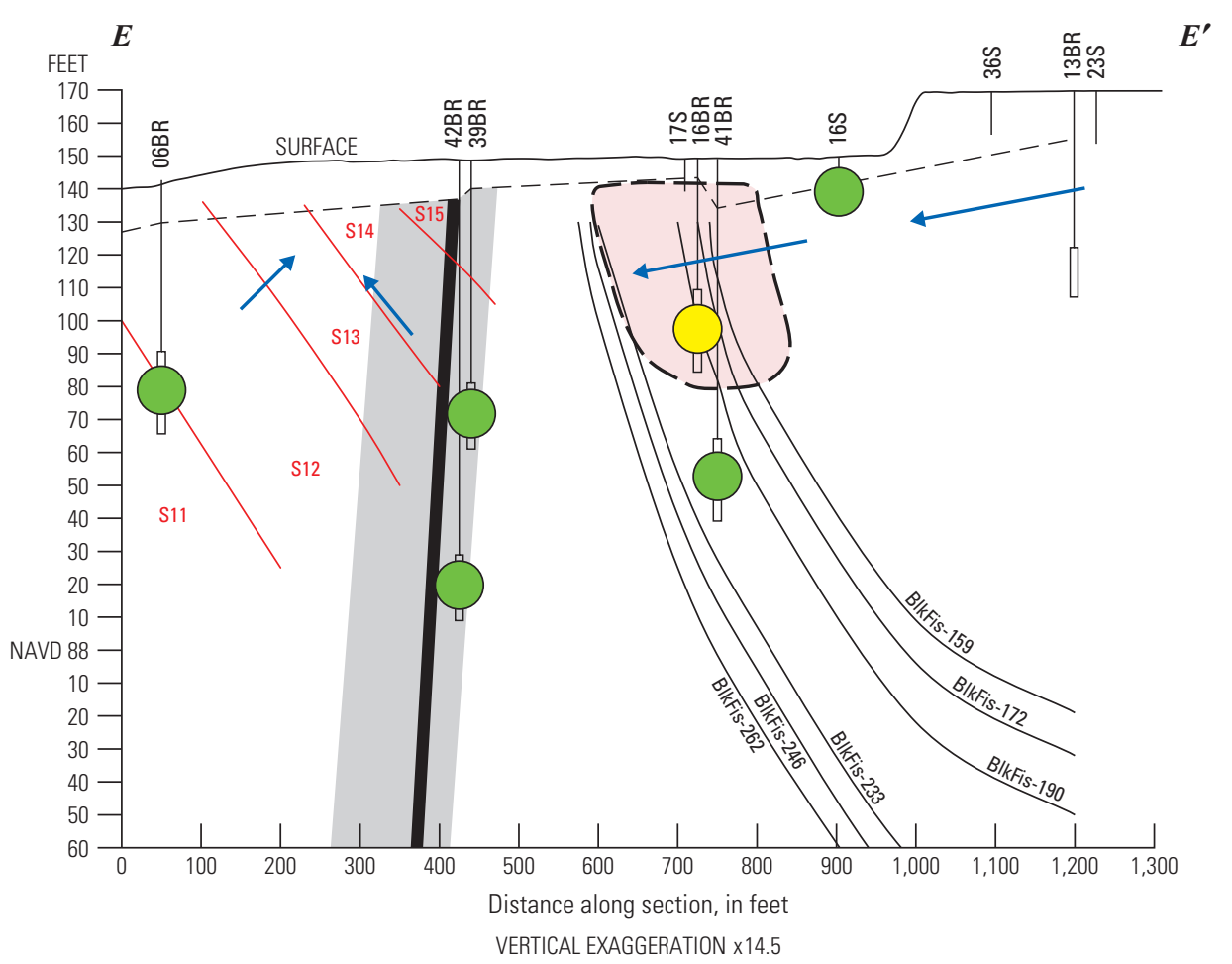

VC concentration, in $\mu \mathrm{g} / \mathrm{L}$ 


\section{A. 2014 VC concentrations}

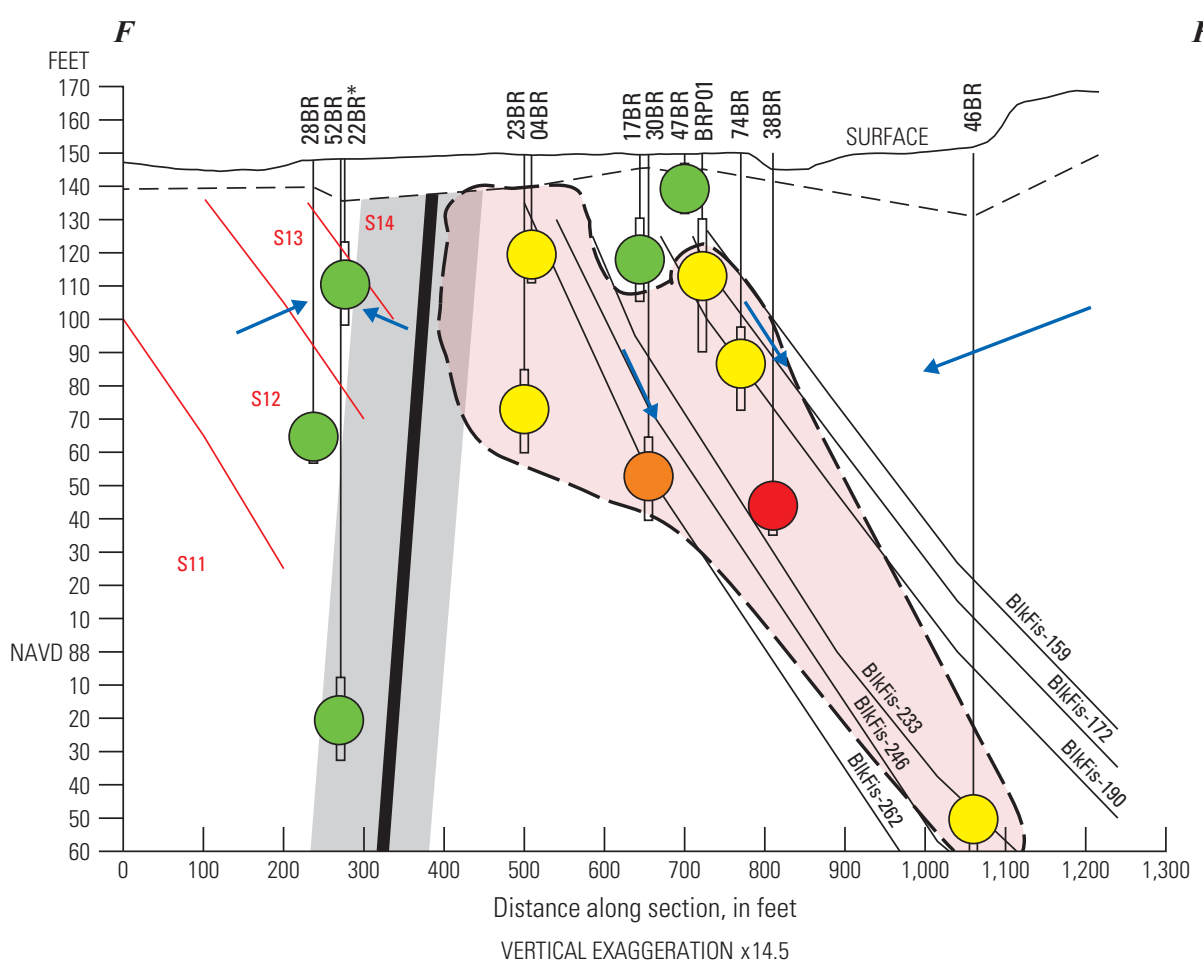

\section{B. 2015-17 VC most recent concentrations}

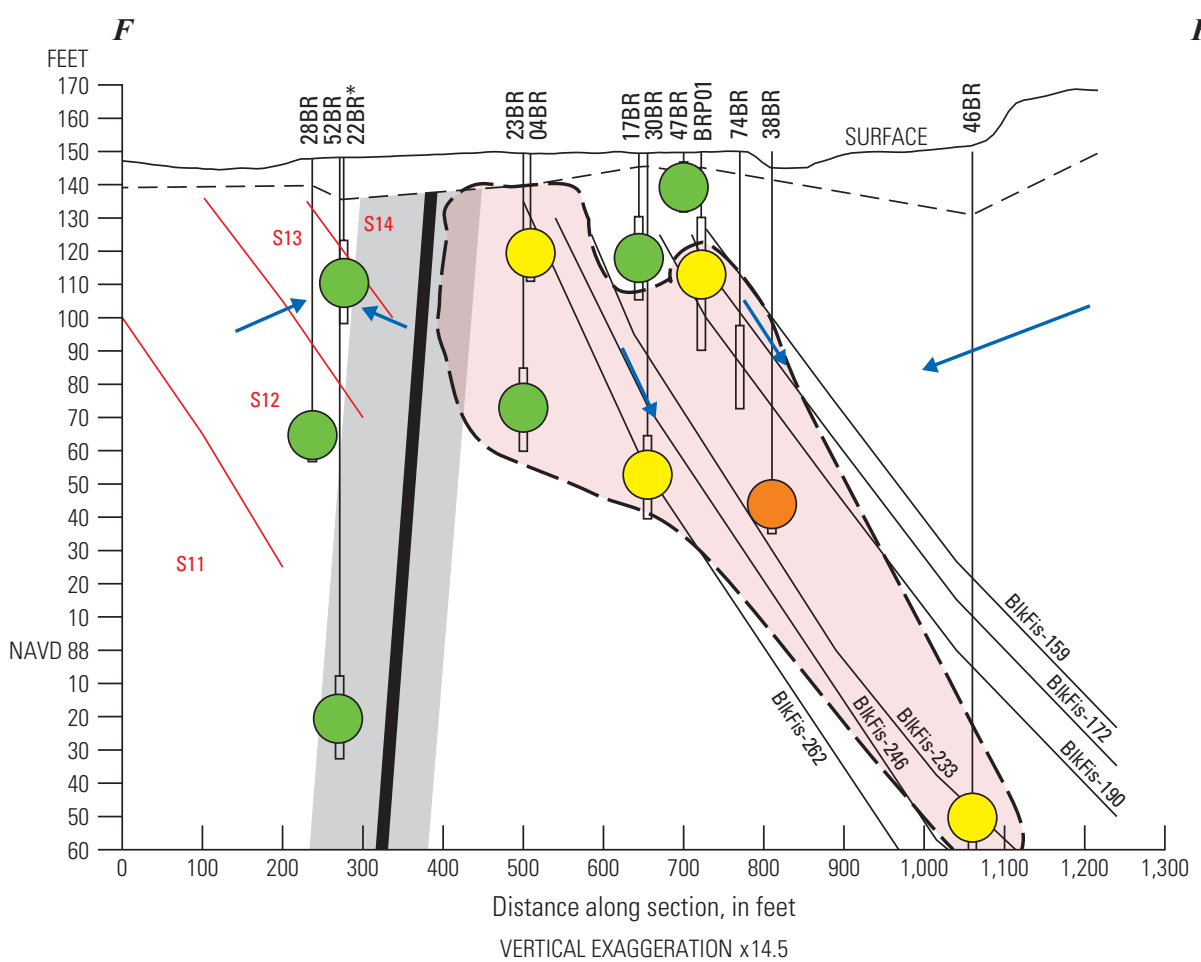

$\boldsymbol{F}^{\prime}$

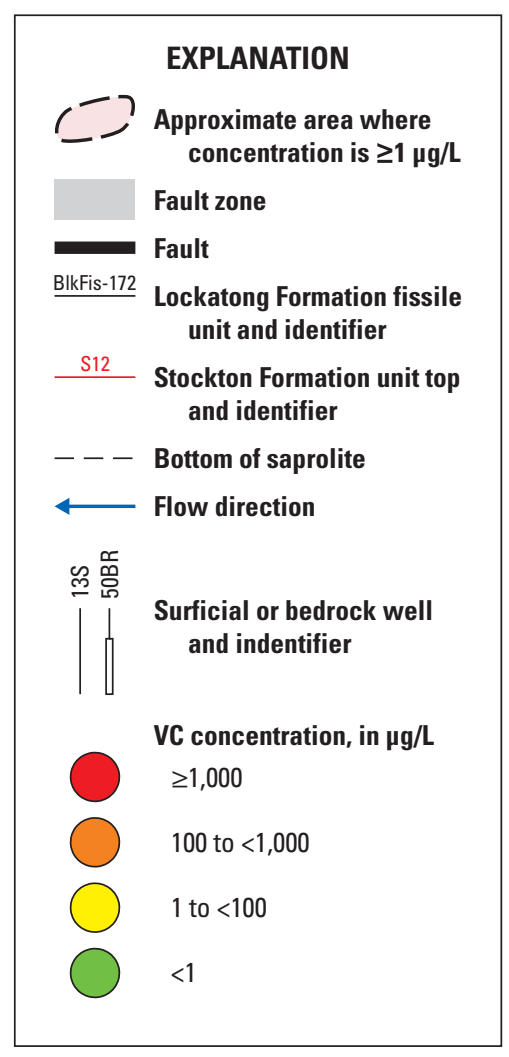

Figure 39. Vertical extent of vinyl chloride concentrations in wells along section $\mathrm{F}-\mathrm{F}^{\prime}$ in $A, 2014$ and $B, 2015-17$, former Naval Air Warfare Center, West Trenton, New Jersey. (VC, vinyl chloride; mg/L, micrograms per liter) 


\section{A. 2014 VC concentrations}

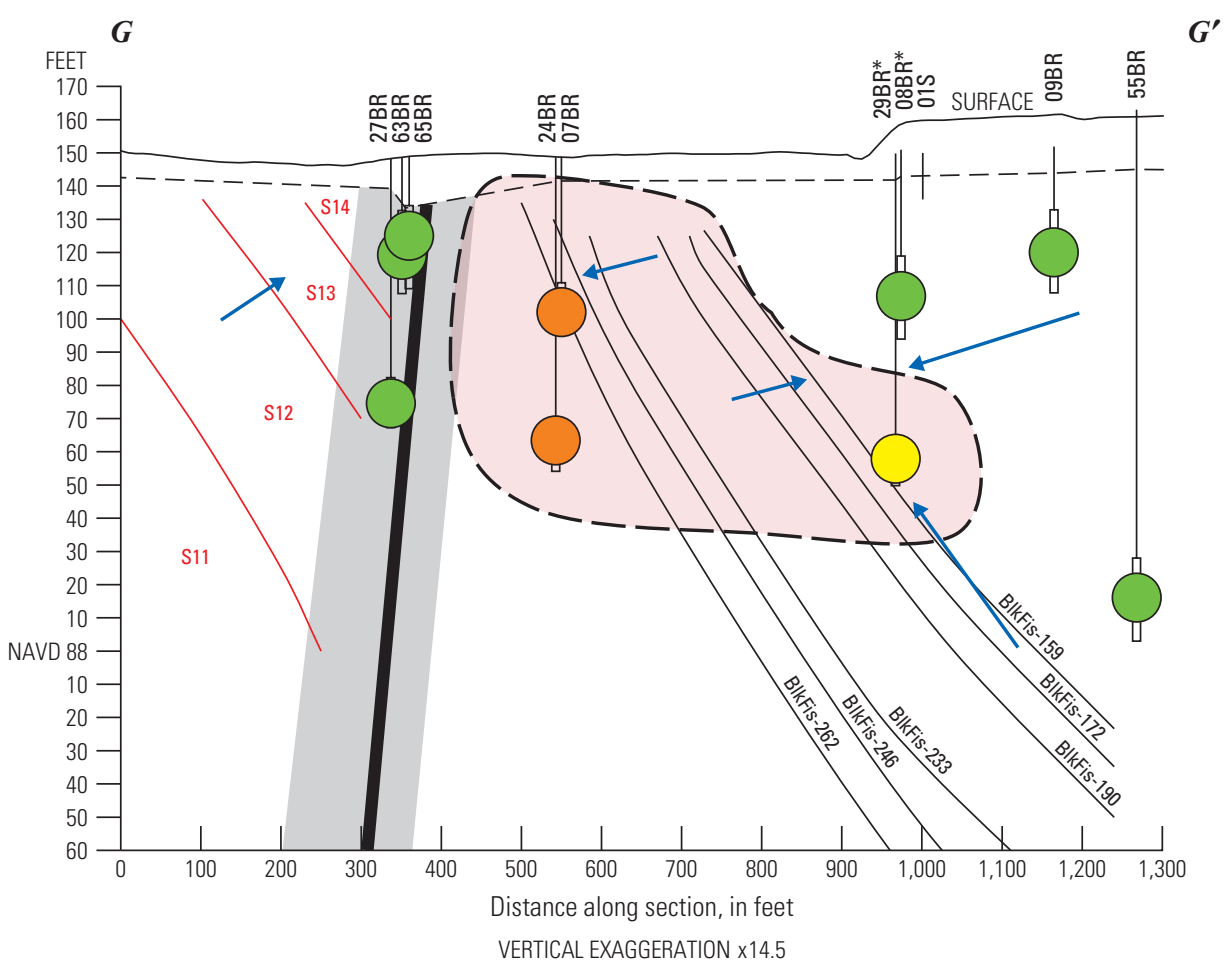

\section{B. 2015-17 VC most recent concentrations}

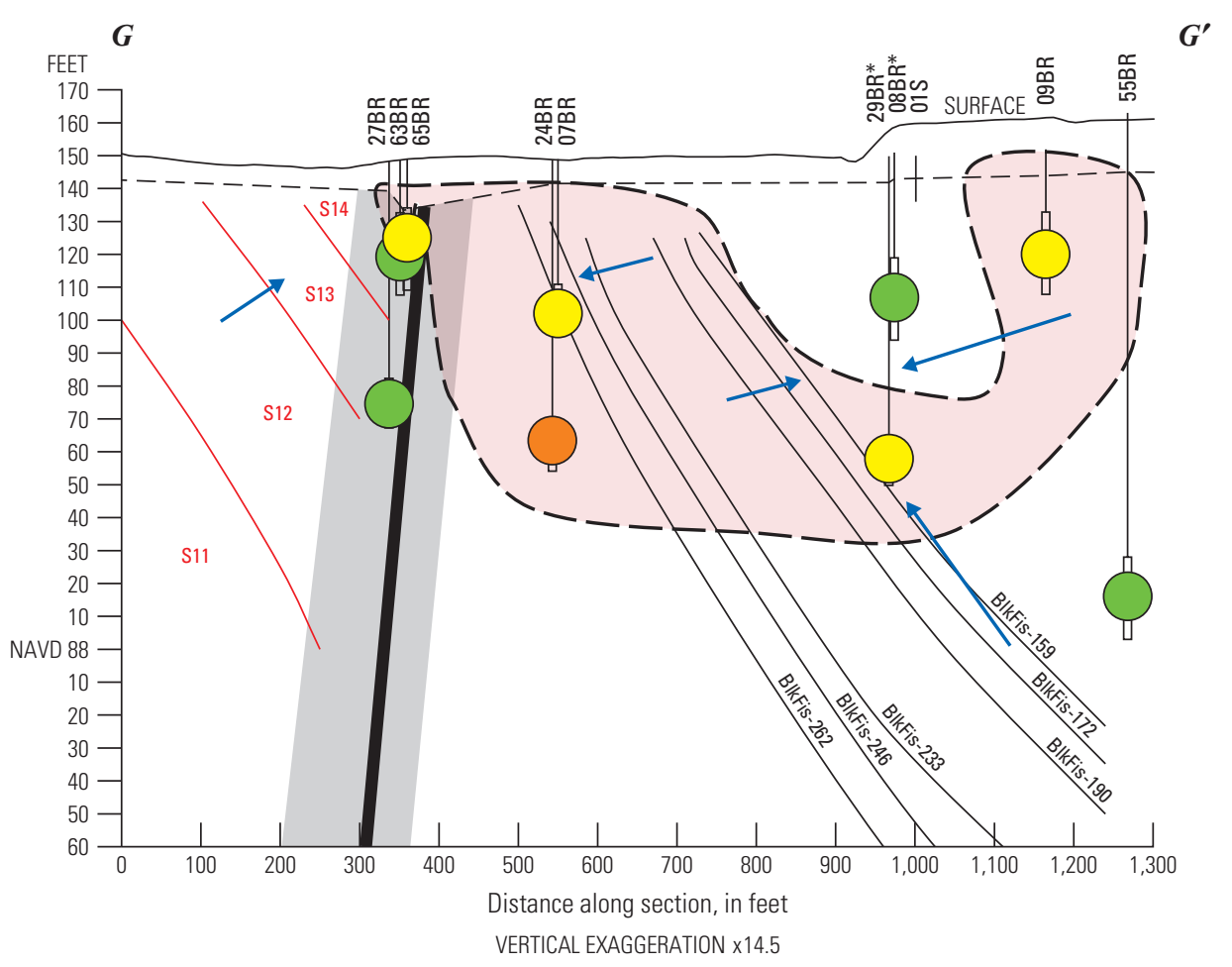

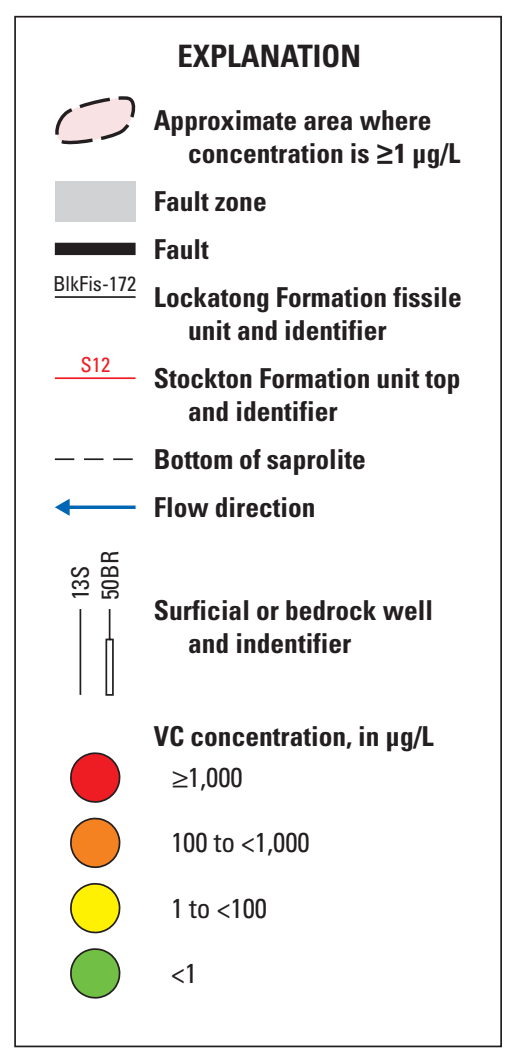

Figure 40. Vertical extent of vinyl chloride concentrations in wells along section $G^{-} G^{\prime}$ in $A, 2014$ and $B, 2015-17$, former Naval Air Warfare Center, West Trenton, New Jersey. (VC, vinyl chloride; mg/L, micrograms per liter) 


\section{A. 2014 VC concentrations}

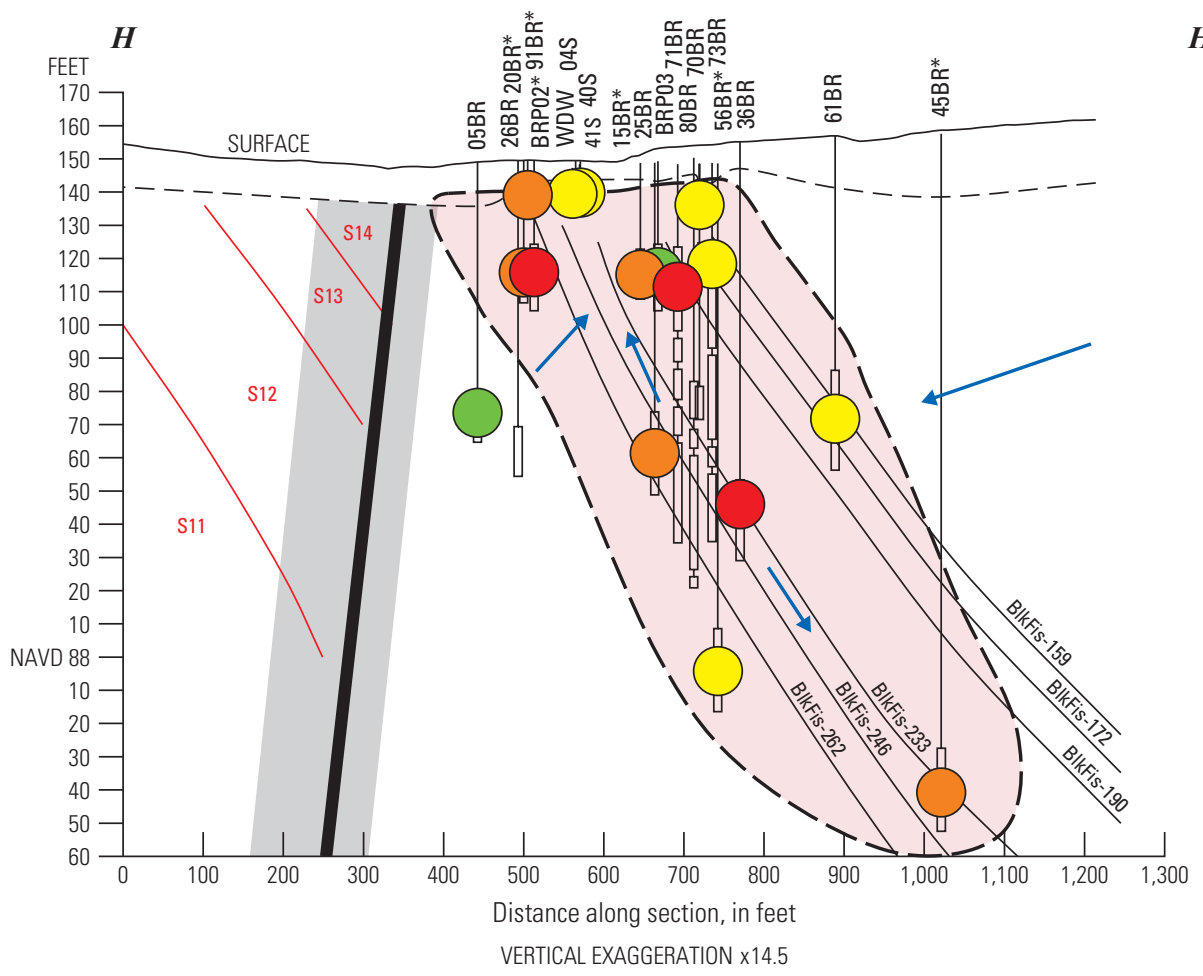

\section{B. 2015-17 VC most recent concentrations}

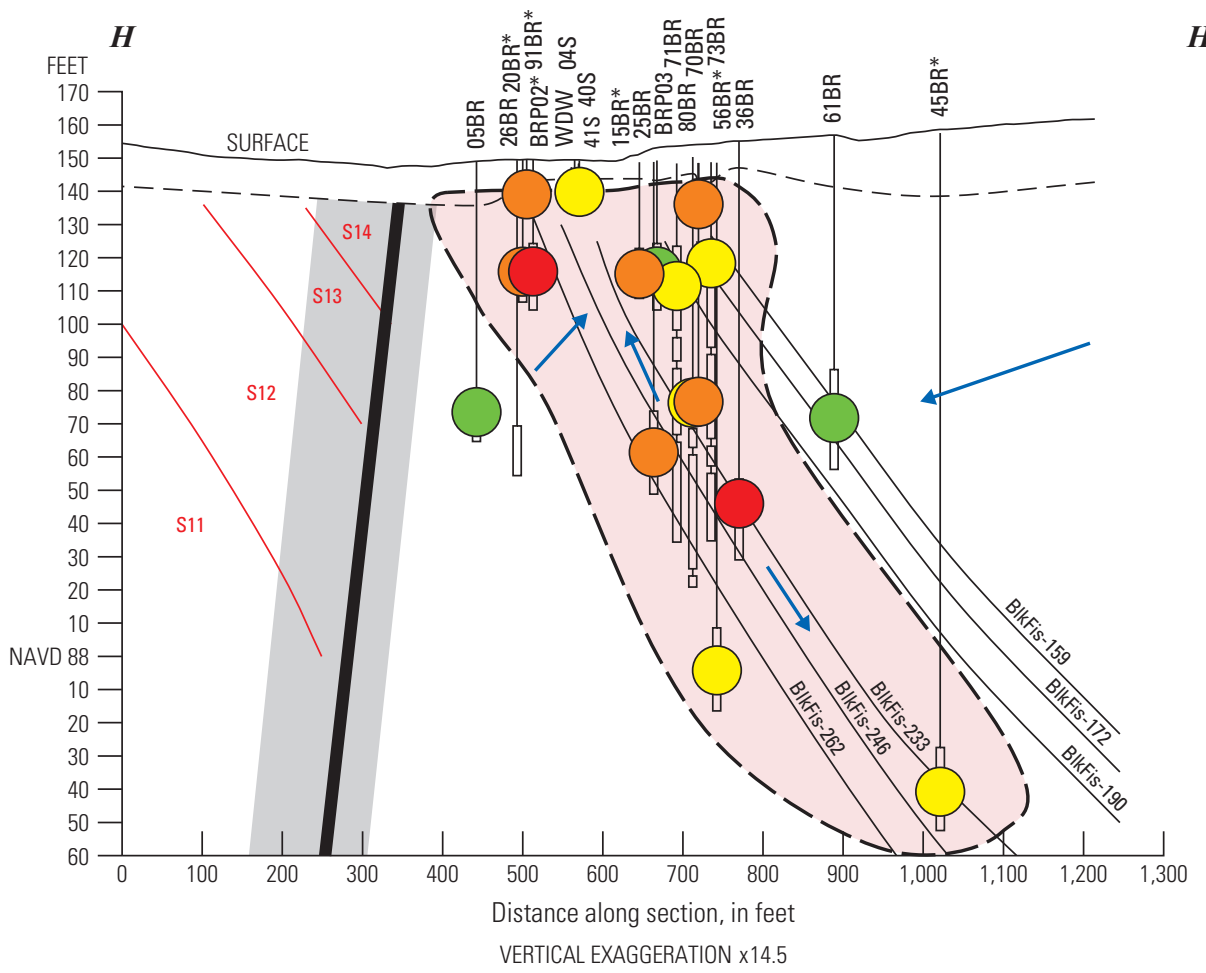

$\boldsymbol{H}^{\prime}$

Approximate area where
concentration is $\geq 1 \mu \mathrm{g} / \mathrm{L}$
Fault zone
Fault
Lockatong Formation fissile
unit and identifier
Stockton Formation unit top
and identifier

Figure 41. Vertical extent of vinyl chloride concentrations in wells along section $\mathrm{H}-\mathrm{H}^{\prime}$ in $A, 2014$ and $B, 2015-17$, former Naval Air Warfare Center, West Trenton, New Jersey. (VC, vinyl chloride; mg/L, micrograms per liter) 


\section{A. 2014 VC concentrations}

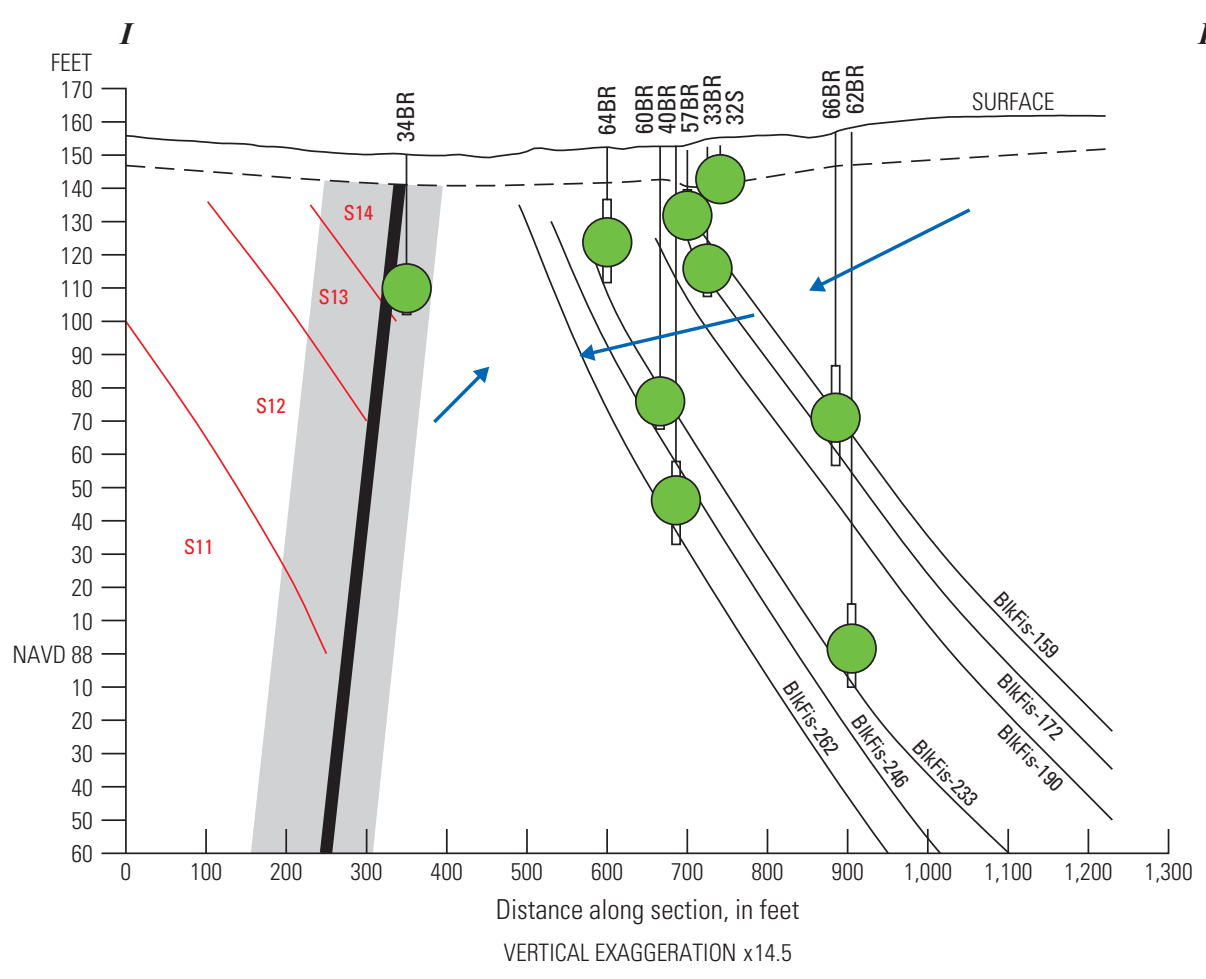

\section{B. 2015-17 VC most recent concentrations}

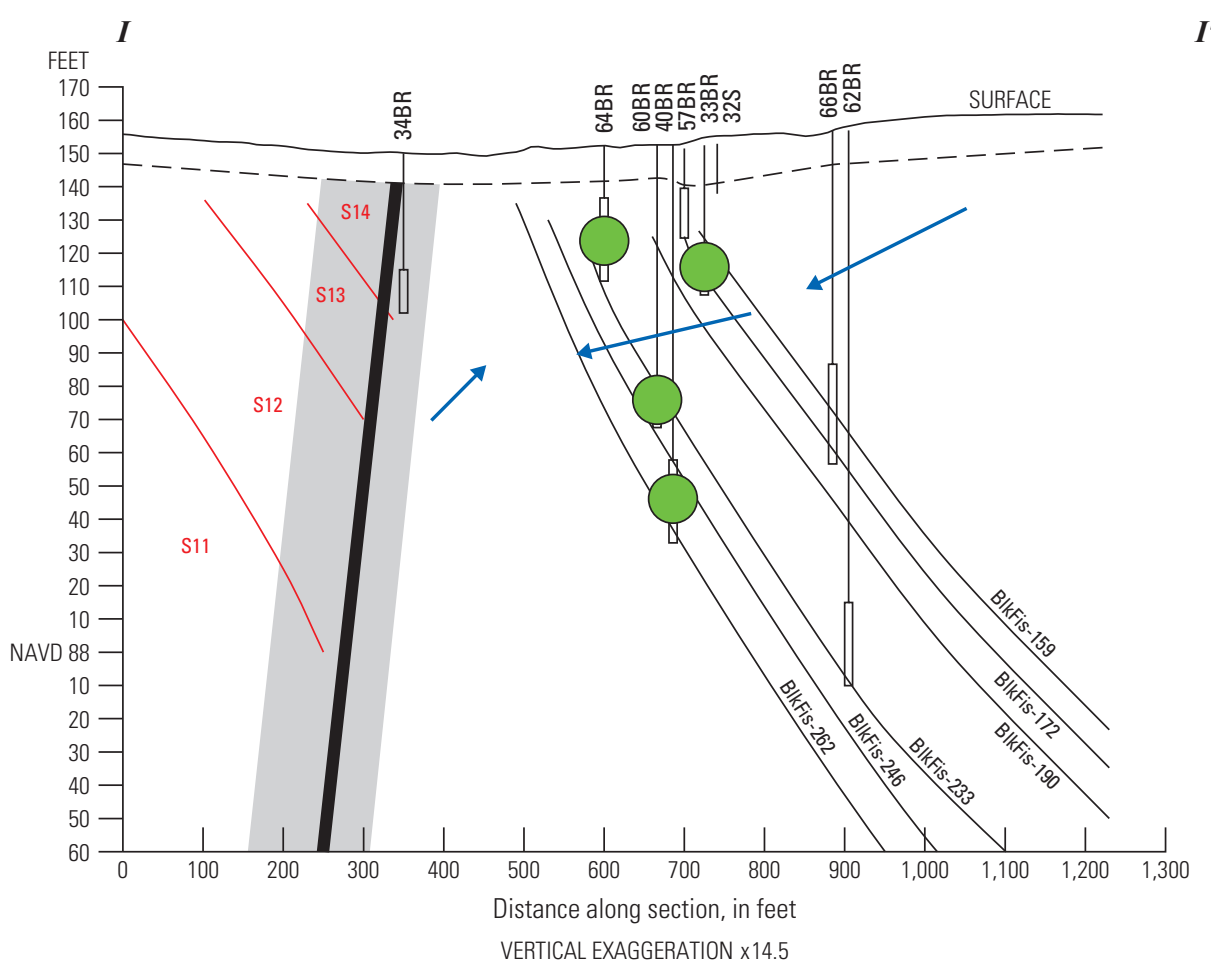

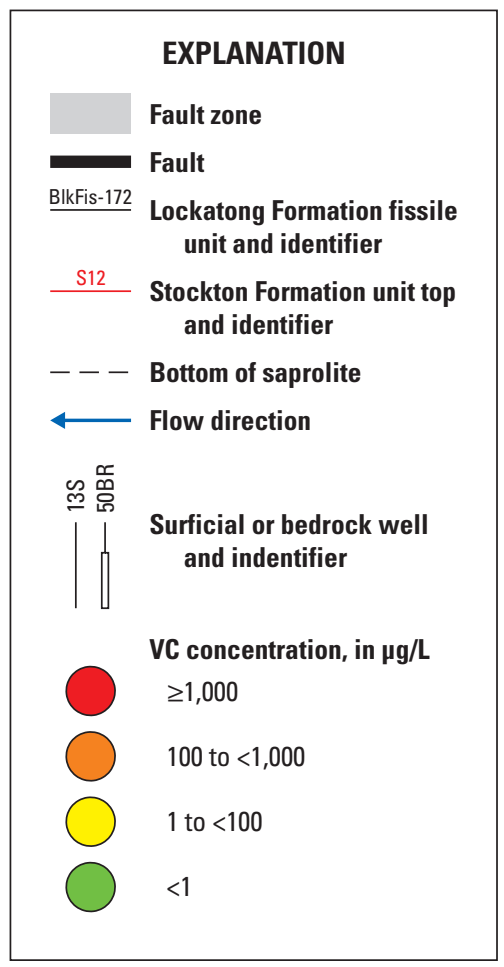

Figure 42. Vertical extent of vinyl chloride concentrations in wells along section I-I' in A, 2014 and B, 2015-17, former Naval Air Warfare Center, West Trenton, New Jersey. (VC, vinyl chloride; mg/L, micrograms per liter) 
decreased to $<1 \mu \mathrm{g} / \mathrm{L}$ the last time the wells were sampled in 2016 (fig. 43). Well 50BR had measurable TCE and cisDCE concentrations of 3.3 and $2.2 \mu \mathrm{g} / \mathrm{L}$, respectively, in 2016, but both VOCs also had recent slight downward trends over time. This well is to the east of the railroad tracks (the eastern boundary of NAWC), so these results indicate that though TCE and cisDCE concentrations are migrating offsite to the northeast of NAWC, the concentrations are decreasing with time.

Trends in TCE, cisDCE, and VC concentrations were stable or gradually downward over time in wells $11 \mathrm{~S}, 12 \mathrm{~S}$, 42S, 29S, 03BR, 32BR, and 35S along section B-B' (fig. 44). All VOCs in these wells had decreased to $<1 \mu \mathrm{g} / \mathrm{L}$ as of their last sampling from 2014 to 2016. Wells 12BR and 28S also had downward trends for all VOCs over time, and both wells had low VOC concentrations (all compounds $<5.8 \mu \mathrm{g} / \mathrm{L}$ ) from 2014 to 2016. Well 51BR had a downward concentration trend for all VOCs, with TCE and cisDCE concentrations of $<1.4$ $\mu \mathrm{g} / \mathrm{L}$ and $<6.8 \mu \mathrm{g} / \mathrm{L}$, respectively, in 2016. Although concentrations of all VOCs in well 02BR varied, overall concentrations remained about the same from 1990 to 2016.

Wells 14BR, 30S, 11MW1, and 37BR, along section $\mathrm{C}-\mathrm{C}^{\prime}$ had stable to gradually downward trending VOC concentrations since they were included in the long-term monitoring plan (fig. 45). All these wells had VOC concentrations $<1 \mu \mathrm{g} / \mathrm{L}$ as of their last sampling. Wells $31 \mathrm{BR}$ and $31 \mathrm{~S}$ had downward trends in TCE and cisDCE with concentrations of each $<10$ $\mu \mathrm{g} / \mathrm{L}$ in 2016. Since 2010, well 54BR had a downward trend in TCE concentrations but an upward trend in cisDCE concentrations, indicating some ongoing anaerobic biodegradation in this well. Well 48BR, the extraction well in the Site 3 area of NAWC, had a very stable to gradually downward trend in TCE, cisDCE, and VC concentrations from 1998 to 2017.

All wells along section D-D' (43BR, 44BR, 53BR, 49BR, 37S, 34S and 19BR) had stable or downward concentration trends for all VOCs since they were included in the long-term monitoring plan (fig. 46). Concentrations of TCE, cisDCE, and VC were $<1 \mu \mathrm{g} / \mathrm{L}$ in all these wells as of their last sampling in 2014 or 2016.

\section{Site 1 Western Sections E-E' to I-I'}

All wells along section E-E' (16S, 41BR, 16BR, 39BR, 42BR, and 06BR) had stable or downward trends for all VOCs since they were included in the long-term monitoring plan (fig. 47). All compound concentrations were $<1.2 \mu \mathrm{g} / \mathrm{L}$ in wells $16 \mathrm{~S}, 39 \mathrm{BR}, 42 \mathrm{BR}$, and 06BR as of their last sampling in 2016. Wells 41BR and 16BR were injection wells that were part of the 2005 bioaugmentation study conducted by the Navy, ECOR Solutions, and Geosyntec Consultants from 2005 to 2008 (Geosyntec Consultants, 2010). Concentrations of TCE dropped precipitously in well 41BR from 670 to $<1 \mu \mathrm{g} / \mathrm{L}$ and in well $16 \mathrm{BR}$ from 290 to $<1 \mu \mathrm{g} / \mathrm{L}$ during the first 6 months following the bacteria and ESO injection and have remained at low levels since then. Concentrations of cisDCE and VC also dropped initially in 2005 in both wells but rebounded in 2008 (fig. 47) to an order of magnitude less or about the same as they had been before the injection. The concentrations of cisDCE and $\mathrm{VC}$ have either remained stable or have been decreasing since 2008 in wells 41BR and 16BR.

Along section F-F', wells 52BR, 28BR, 35MW1, and $35 \mathrm{MW}$, all had VOC concentrations $<1 \mathrm{mg} / \mathrm{L}$ during the 2014-17 period. Wells 46BR, 74BR, 47BR, 17BR, 04BR, 52BR, and 28BR had stable or gradual downward concentration trends for TCE, cisDCE, and VC since they were included in the long-term monitoring plan (fig. 48). Wells BRP01 and 38BR were injection wells for bacteria and ESO during the 2005 bioaugmentation study (Geosyntec Consultants, 2010). TCE concentrations in well 38BR decreased rapidly from $16,000 \mu \mathrm{g} / \mathrm{L}$ to $8.1 \mu \mathrm{g} / \mathrm{L}$ in the first 6 months after the injection. TCE concentrations in well BRP01 also decreased from 210 to $<2.7 \mu \mathrm{g} / \mathrm{L}$ in the first 6 months after the injection.

TCE concentrations in these two wells remained below these levels in the ensuing years. Concentrations of cisDCE and $\mathrm{VC}$ in these two wells dropped initially but rebounded as the biodegradation proceeded (fig. 48). As of 2017, concentrations of cisDCE and VC were just slightly less than they were prior to the 2005 bioaugmentation injection.

Well 30BR received an injection of ESO in 2008 as a continuation of the 2005 experiment. TCE concentrations decreased in this well from 17,000 to $<1 \mu \mathrm{g} / \mathrm{L}$ within 1 year and have remained low (fig. 38). Concentrations of cisDCE and $\mathrm{VC}$ rebounded slightly after about 6 years but were much lower in 2017 than prior to the 2008 ESO injection. Well 22BR has generally exhibited a gradually downward trend in all VOC concentrations except for a large increase in all VOCs in 2012. The cause for this increase is unknown, but concentrations returned to their pre-2012 levels, and the downward trend has been re-established. Well 23BR had decreasing VOC concentrations until 2009-10 when the well water was removed as part of a diffusion experiment. The groundwater that refilled this well as it recovered had much higher VOC concentrations. Since 2012, the concentrations of all VOCs have been declining from those higher levels.

Along section $\mathrm{G}-\mathrm{G}^{\prime}$, wells 55BR, 27BR, 33S, and 35BR, remained uncontaminated throughout the 2014-17 period. Wells 09BR, 21BR, 63BR, and 65BR, had stable to gradually downward concentration trends for TCE, cisDCE, and VC since they were included in the long-term monitoring plan (fig. 49). Wells 07BR and 24BR remained highly contaminated and had stable to gradually downward concentration trends for all three VOCs for many years prior to 2017. Extraction well 08BR was highly contaminated and has had relatively stable TCE and cisDCE concentrations since 2012. Extraction well 29BR is one of the few wells at NAWC that had upward TCE, cisDCE, and VC concentration trends since 2012. This well has started drawing in more highly contaminated groundwater since 2012. A possible explanation may be that the area of the drawdown cone for well 29BR has spread to intersect new, more highly contaminated fractures in the aquifer previously not under the influence of its pumping. 
Along section $\mathrm{H}-\mathrm{H}^{\prime}$, wells $05 \mathrm{BR}$ and BRP03 are the only wells with $<1 \mu \mathrm{g} / \mathrm{L}$ concentrations of TCE, cisDCE and VC (fig. 50). Wells 40S, 70BR-10, 70BR-72, 73BR-A, 71BR-A, 80BR-A, 61BR, WDW, and 91BR have had stable or gradually downward concentration trends for TCE since they were included in the long-term monitoring plan (fig. 50). Elevated TCE concentrations $(\geq 1,000 \mu \mathrm{g} / \mathrm{L})$ in extraction wells $45 \mathrm{BR}$ and 56BR varied mostly owing to pump malfunction problems but have remained stable since the pumps were repaired. Extraction wells 15BR, 20BR, and BRP02 had high TCE and cisDCE concentrations $(\geq 1,000 \mu \mathrm{g} / \mathrm{L})$, and all have had gradual downward trends for both compounds over time. Well 25BR had increasing VOC concentrations during 2008-17, but this is probably due to its being part of a diffusion study from 2009 to 2010 , during which the water in the well was replaced.

Well 36BR, once the well with the highest TCE concentrations $(\geq 100,000 \mu \mathrm{g} / \mathrm{L})$ at the NAWC site, had TCE concentrations $<10 \mu \mathrm{g} / \mathrm{L}$ in 2017 (fig. 50). This precipitous drop was owing to its use as an injection well for the 2008 bioaugmentation study when it received additional bacteria and ESO as a bacterial food source (Shapiro and others, 2018). The TCE concentrations dropped 3-4 orders of magnitude immediately after the injection in 2008 and remained at this level thereafter. Since the bioaugmentation injection, cisDCE and VC concentrations have increased because they were produced as biodegradation of TCE proceeded. Though still ongoing, the anaerobic biodegradation has "stalled" so all the cisDCE has not degraded to VC and ethene. This phenomenon is frequently found at sites where anaerobic biodegradation of TCE is occurring (Wiedemeier and others, 1998).

Most wells along section I-I' to the west of the NAWC property had TCE, cisDCE, and VC concentrations of $<1$ $\mu \mathrm{g} / \mathrm{L}$ (fig. 51). Only wells 60BR and 40BR, a well doublet in the parking lot near the NAWC treatment plant, had TCE and cisDCE concentrations $>1 \mu \mathrm{g} / \mathrm{L}$ in 2017. In well 60BR (85 $\mathrm{ft}$ deep), TCE had been decreasing slowly for many years to $120 \mu \mathrm{g} / \mathrm{L}$ in 2013 , but then some variability occurred, and the concentration increased to $350 \mu \mathrm{g} / \mathrm{L}$ by 2017 . The cisDCE concentrations in this well increased from $13 \mu \mathrm{g} / \mathrm{L}$ in 2013 to $96 \mu \mathrm{g} / \mathrm{L}$ by 2017 . The reason for the variability in $60 \mathrm{BR}$ is not immediately clear. In well 40BR (120 ft deep), TCE concentrations had slowly decreased since the late 1990s to $9 \mu \mathrm{g} / \mathrm{L}$ in 2017, but since 2010, cisDCE concentrations increased from $53 \mu \mathrm{g} / \mathrm{L}$ to $130 \mu \mathrm{g} / \mathrm{L}$ in 2017 . These findings indicate that natural anaerobic biodegradation is occurring in this well, with TCE degrading to cisDCE. Overall the results for wells along section I-I' (as well as sections $\mathrm{J}-\mathrm{J}^{\prime}$ and $\mathrm{K}-\mathrm{K}^{\prime}$ ) show that TCE, cisDCE, and $\mathrm{VC}$ in all other wells to the west of NAWC have remained at $<1 \mu \mathrm{g} / \mathrm{L}$, so no VOCs have been transported off site to the south and west.

\section{PFAS Contamination at the Former NAWC Site During 2015-17}

In 2015, the Navy and its contractor sampled only well 48BR on the northeastern side of the former NAWC site for PFAS compounds. PFOS, PFOA, and PFNA were detected at $230 \mathrm{ng} / \mathrm{L}, 37 \mathrm{ng} / \mathrm{L}$, and $6.4 \mathrm{ng} / \mathrm{L}$, respectively, in this well (Watermark Environmental, 2016b; Appendix 2). The PFOS concentration was much greater than the EPA HAL of $70 \mathrm{ng} / \mathrm{L}$ and the NJDEP MCL of $13 \mathrm{ng} / \mathrm{L}$. The PFOA concentration was less than its EPA HAL of $70 \mathrm{ng} / \mathrm{L}$ but above its NJDEP MCL of $14 \mathrm{ng} / \mathrm{L}$. The PFNA concentration was less than its NJDEP MCL of $13 \mathrm{ng} / \mathrm{L}$.

Based on these findings, additional sampling for PFASs was done in 2016 and 2017 (Watermark Environmental, 2017; Tetra Tech, 2018b). The areal extent of PFOS, PFOA, and PFNA contamination was evaluated by plotting the data on sets of maps for 2016 and 2017. The bulk of the PFAS contamination was in the Site 3 area on the eastern side of the NAWC facility (figs. 52-57). Because of the location, the PFOS, PFOA, and PFNA contamination was evaluated vertically in the aquifer by plotting the data on the eastern lines of section A-A' through D-D' only. No graphs of PFAS concentrations over time were plotted because only 1 or 2 years of data were available for most wells.

\section{Areal Extent of PFAS Contamination in Groundwater During 2015-17}

In 2016, the Navy and its contractor sampled the 10 pump-and-treat extraction wells plus 6 wells in the northeastern part of the former NAWC site. PFOS, PFOA, and PFNA were detected in $48 \mathrm{BR}$ at $310 \mathrm{ng} / \mathrm{L}, 48 \mathrm{ng} / \mathrm{L}, 15 \mathrm{ng} / \mathrm{L}$, respectively (Appendix 2). In well 19BR, a well on the Trenton Mercer Airport property (fig. 1), and well 14BR, the well just east of the old NAWC firehouse, all three PFASs were present at $<23 \mathrm{ng} / \mathrm{L}$. However, wells $11 \mathrm{BR}, 13 \mathrm{~S}$, and $14 \mathrm{~S}$ all had $\geq 1,000 \mathrm{ng} / \mathrm{L}$ PFOS, 41-69 ng/L PFOA, and $<13 \mathrm{ng} / \mathrm{L}$ PFNA (figs. 52, 53, and 54). These wells are in the northeastern corner of the NAWC site near the border with the airport property. All other wells sampled in 2016 contained concentrations $<14 \mathrm{ng} / \mathrm{L}$, except for a few estimated concentrations near the detection levels for PFASs in 16S, 91BR, and 22BR (Appendix 2).

The source of the PFAS contamination was not obvious, based on the initial limited sampling results from 2015 and 2016. It is unknown whether the PFAS contamination was due to firefighting activities at the former NAWC site or at the Trenton-Mercer Airport. The former fire chief at NAWC during 1985-95 was contacted to find out if he could recall the use of AFFF at NAWC. The fire chief's recollection was that he had never used AFFF in putting out any actual fires or in any live fire training exercises at NAWC. He did recall, however, that he had tested one 5-gallon drum of AFFF to see how it expanded with the addition of the recommended volume of 
Well 50BR

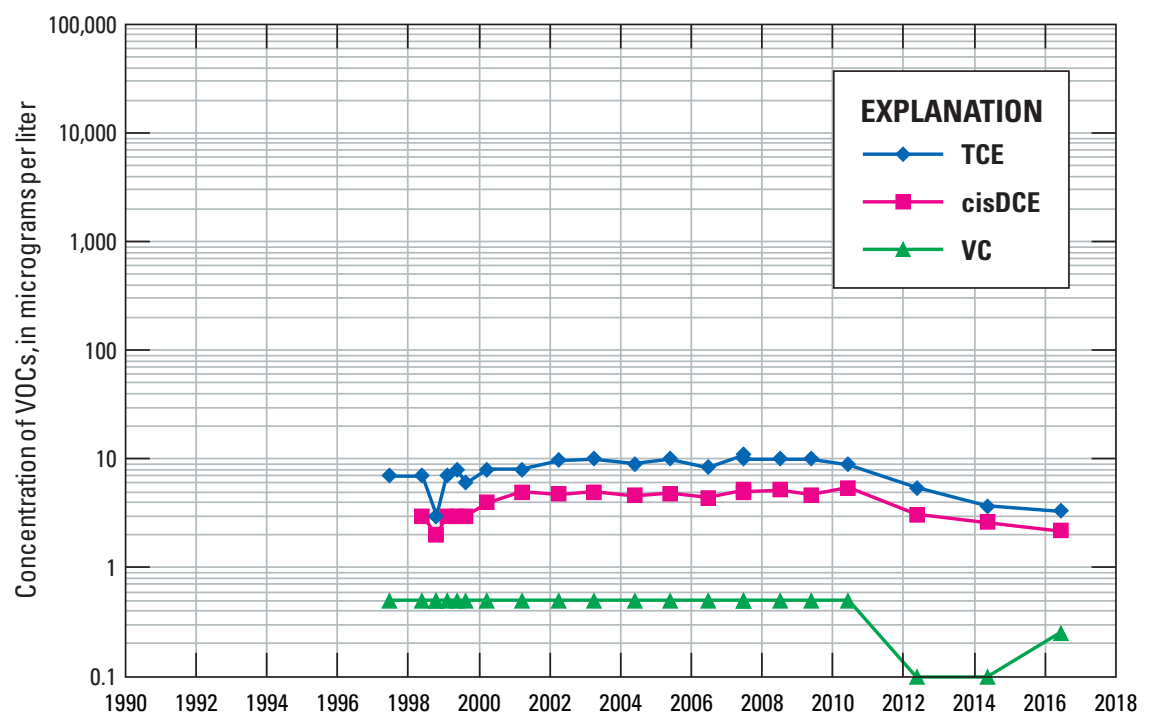

\section{Well 14S}

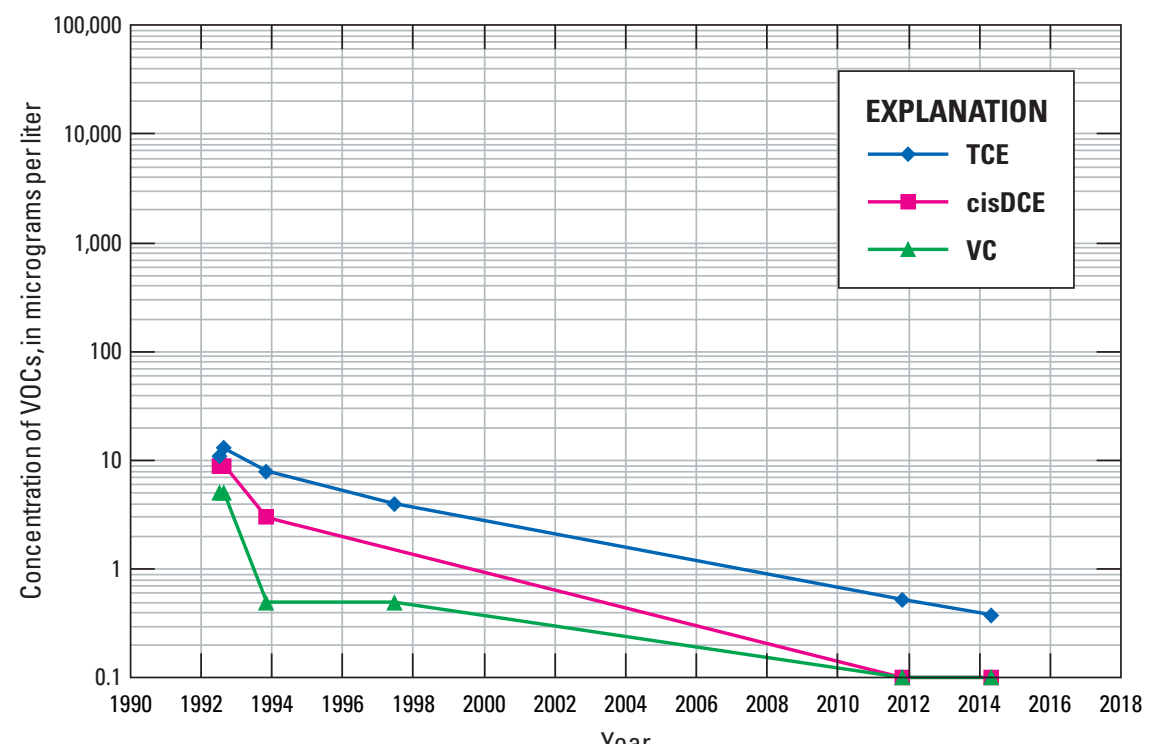

Well 13S

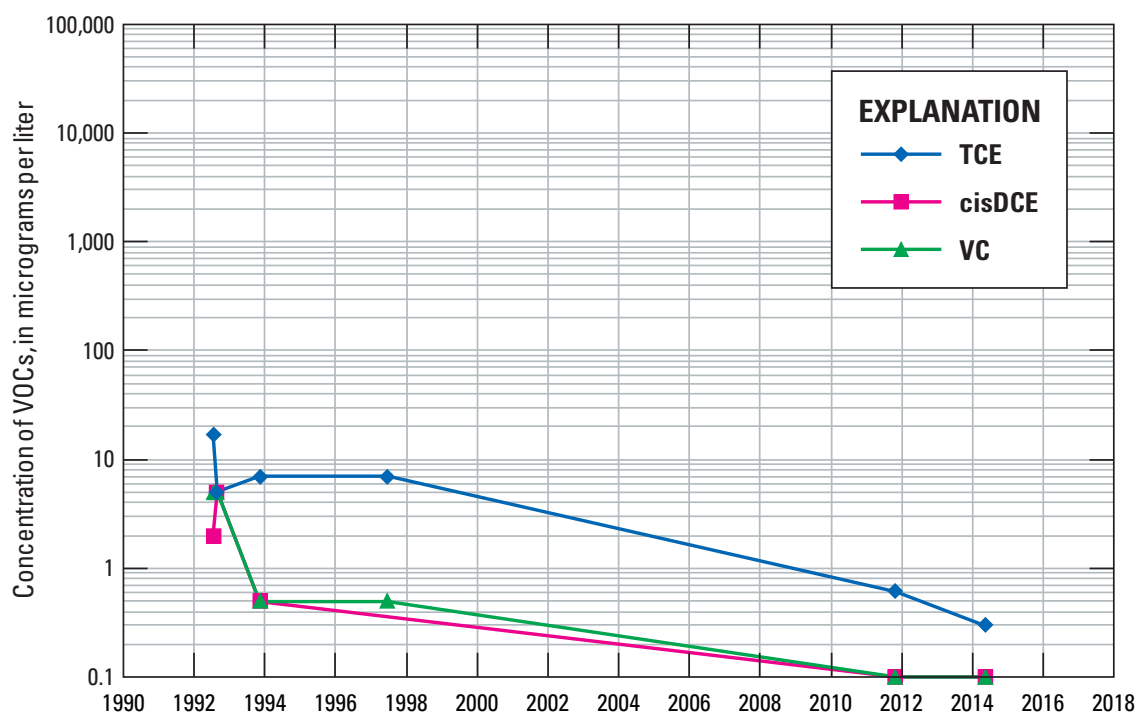

\section{Well 11BR}

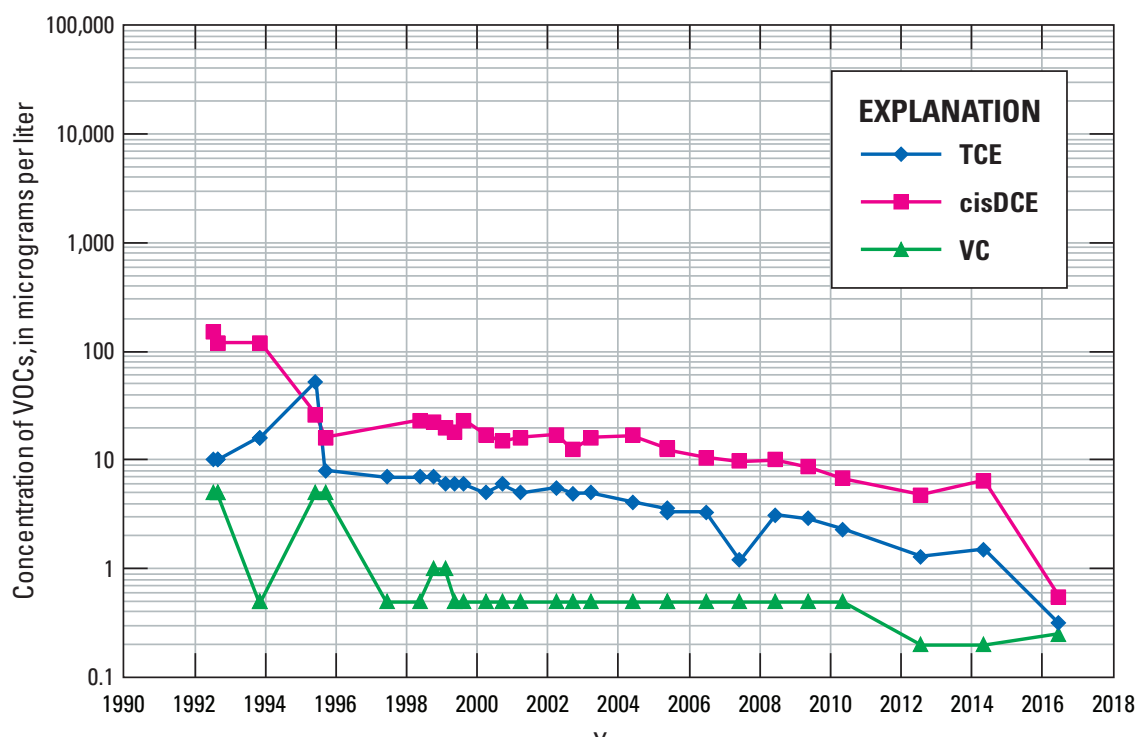

Figure 43. Volatile organic compound concentrations over time in wells along section A-A', former Naval Air Warfare Center, West Trenton, New Jersey, 1992-2017. (VOC, volatile organic compound; TCE, trichloroethene; cisDCE, cis-1,2-dichloroethene; VC, vinyl chloride) 


\section{Well 35S}

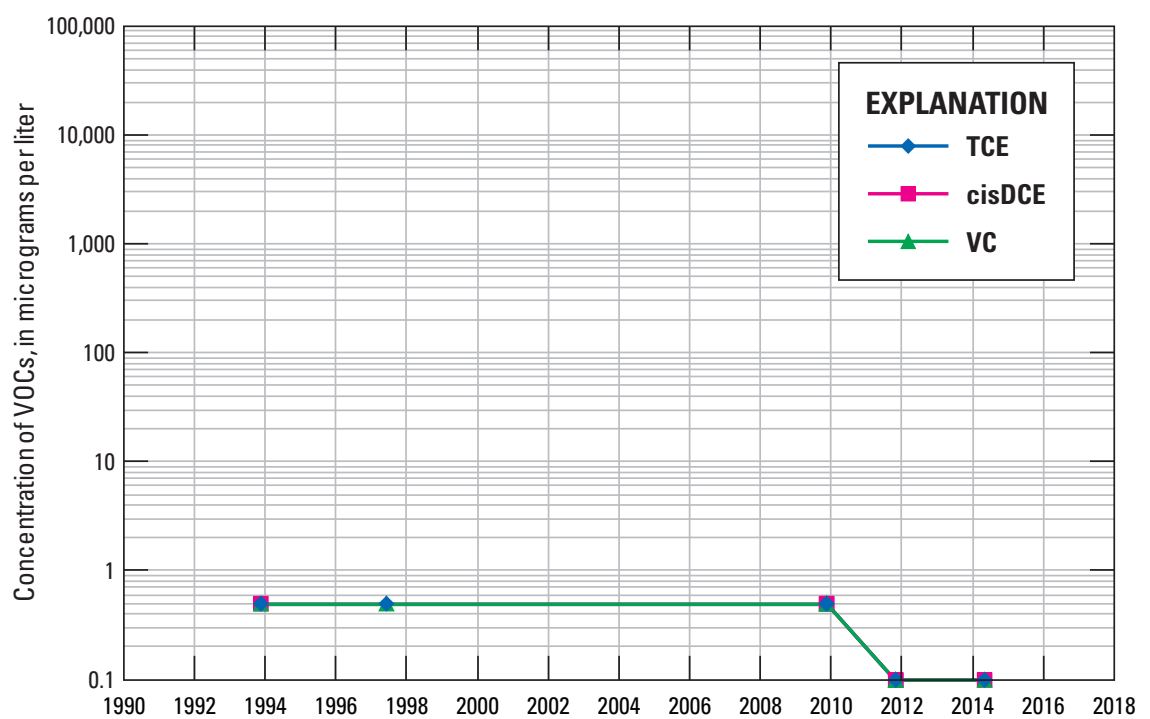

\section{Well 03BR}

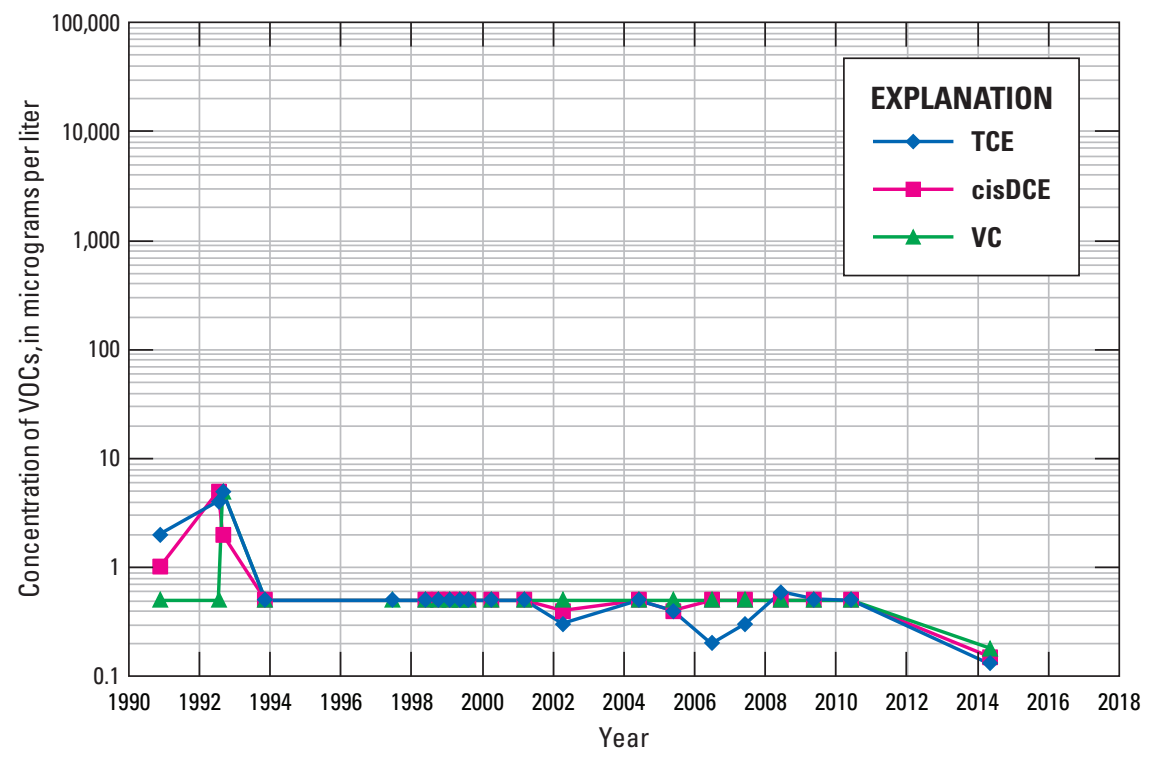

Well 32BR

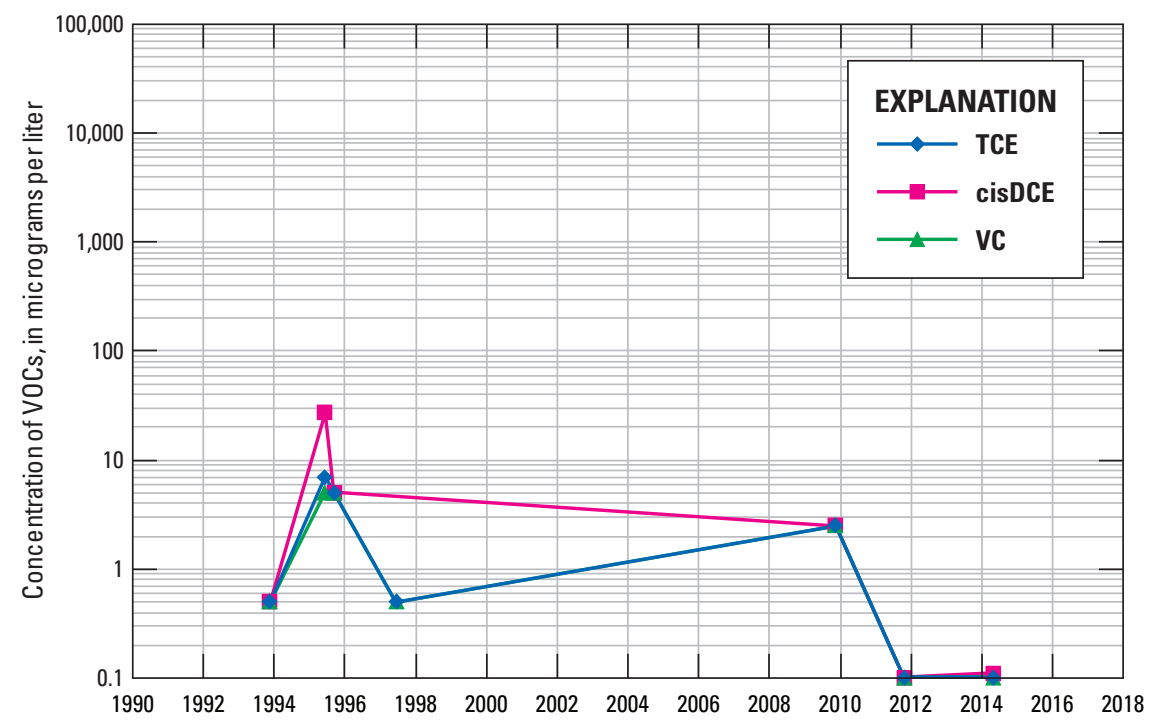

Well $29 S$

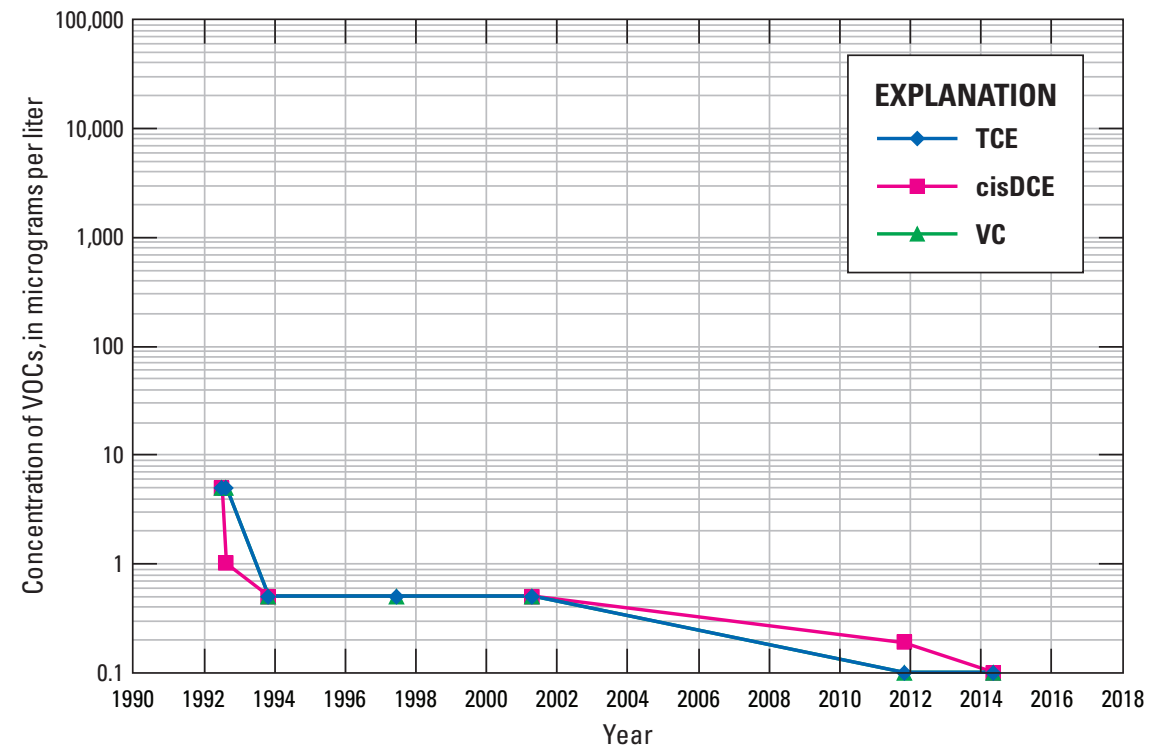

Figure 44. Volatile organic compound concentrations over time in wells along section B-B', former Naval Air Warfare Center, West Trenton, New Jersey, 1990-2017. (VOC, volatile organic compound; TCE, trichloroethene; cisDCE, cis-1,2-dichloroethene; VC, vinyl chloride) 
Well 42S

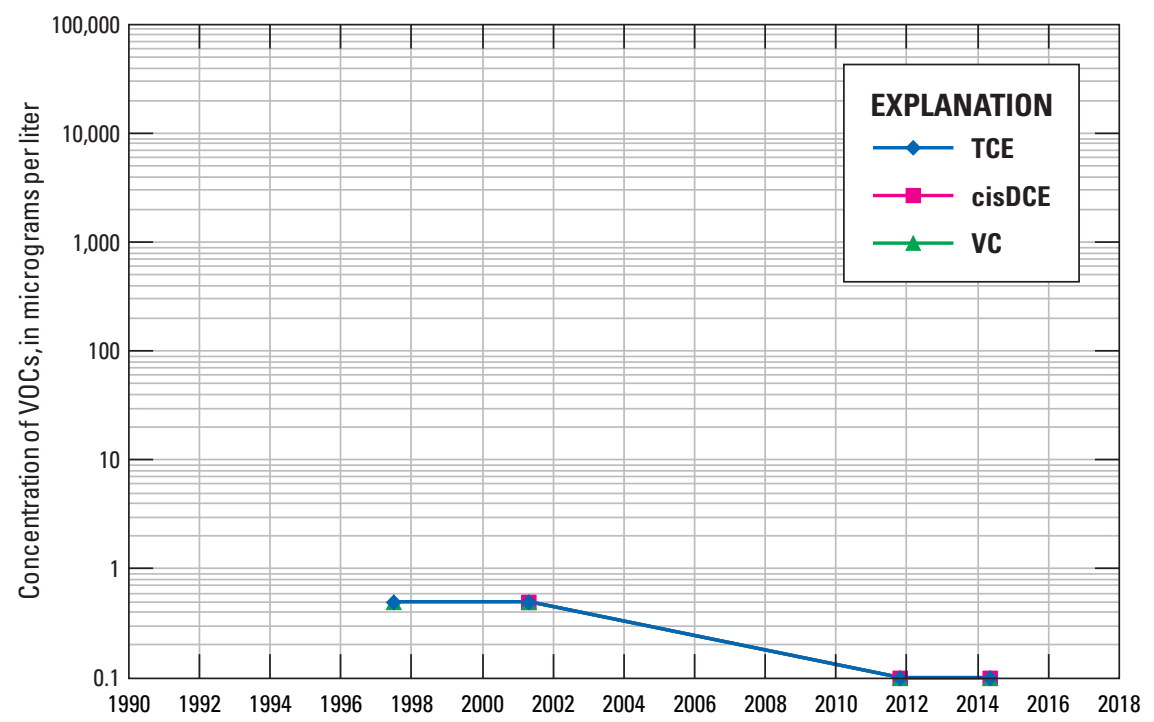

\section{Well 12S}

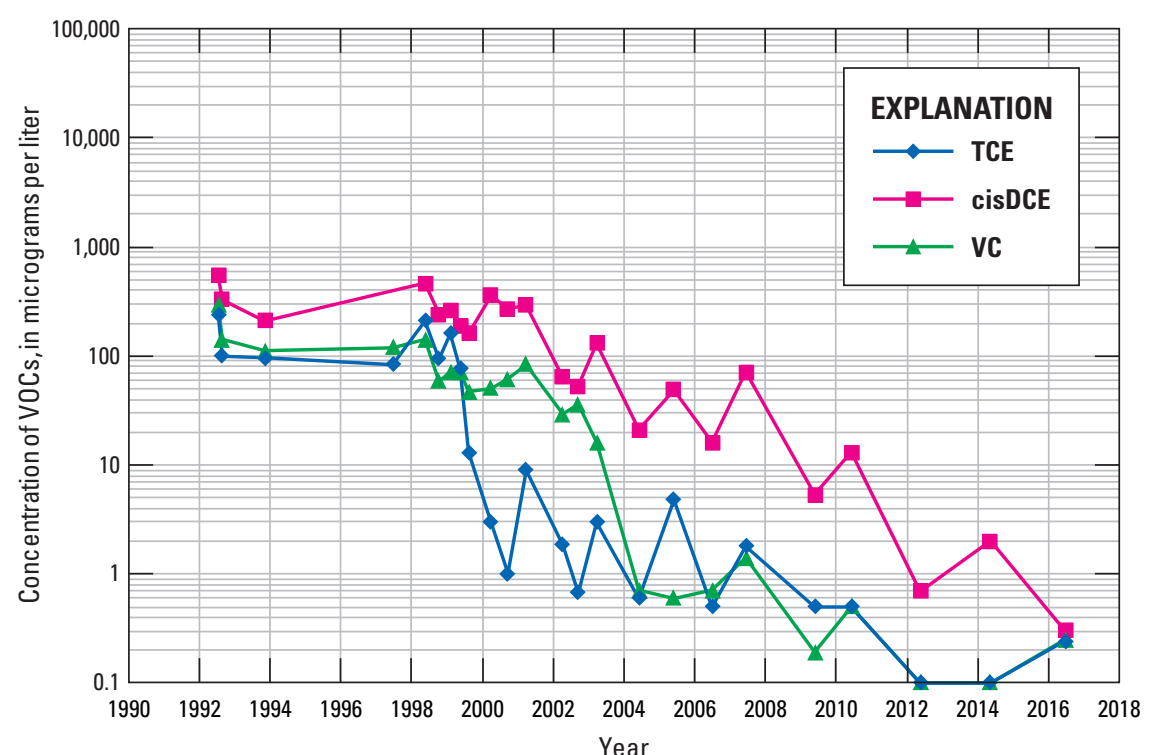

Well 28S

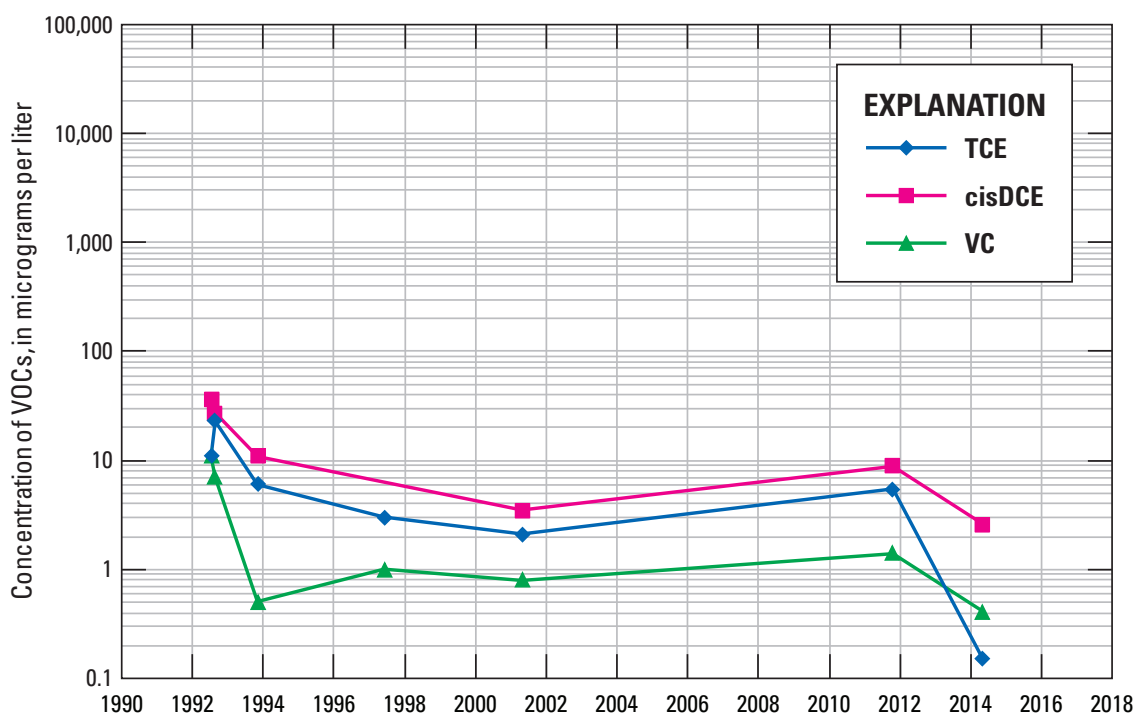

\section{Well 02BR}

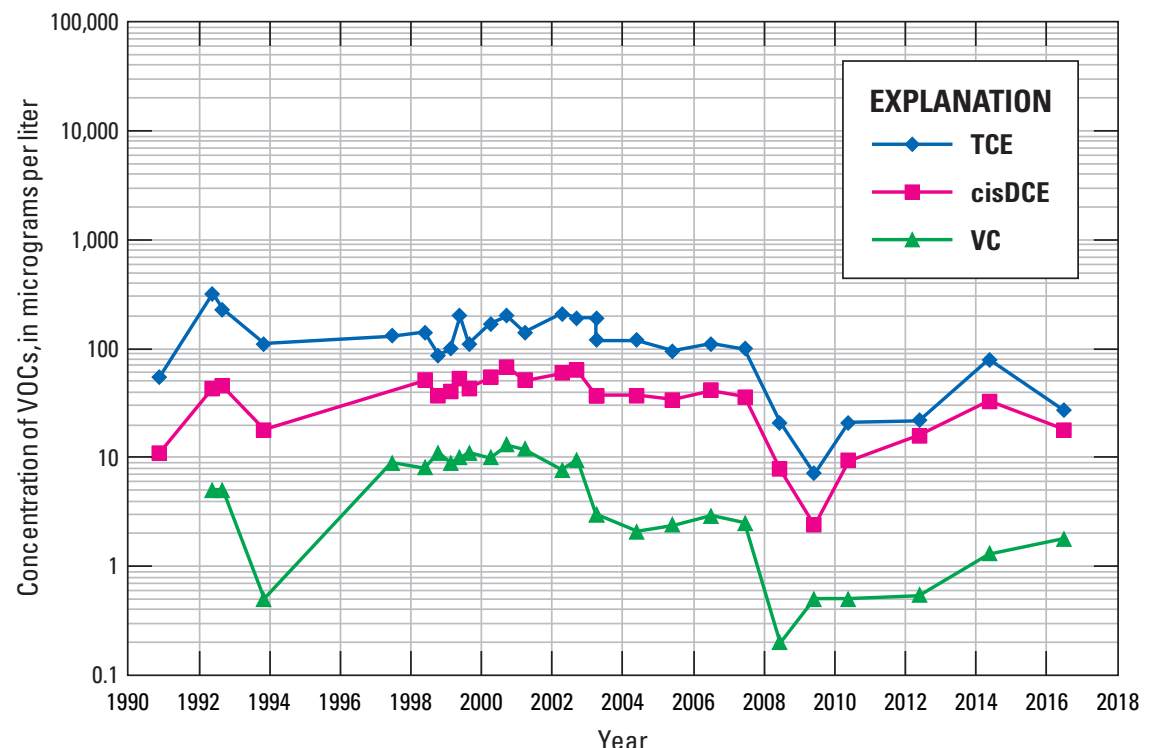

Figure 44. Volatile organic compound concentrations over time in wells along section B-B', former Naval Air Warfare Center, West Trenton, New Jersey, 1990-2017. (VOC, volatile organic compound; TCE, trichloroethene; cisDCE, cis-1,2-dichloroethene; VC, vinyl chloride)—Continued 


\section{Well 51BR}

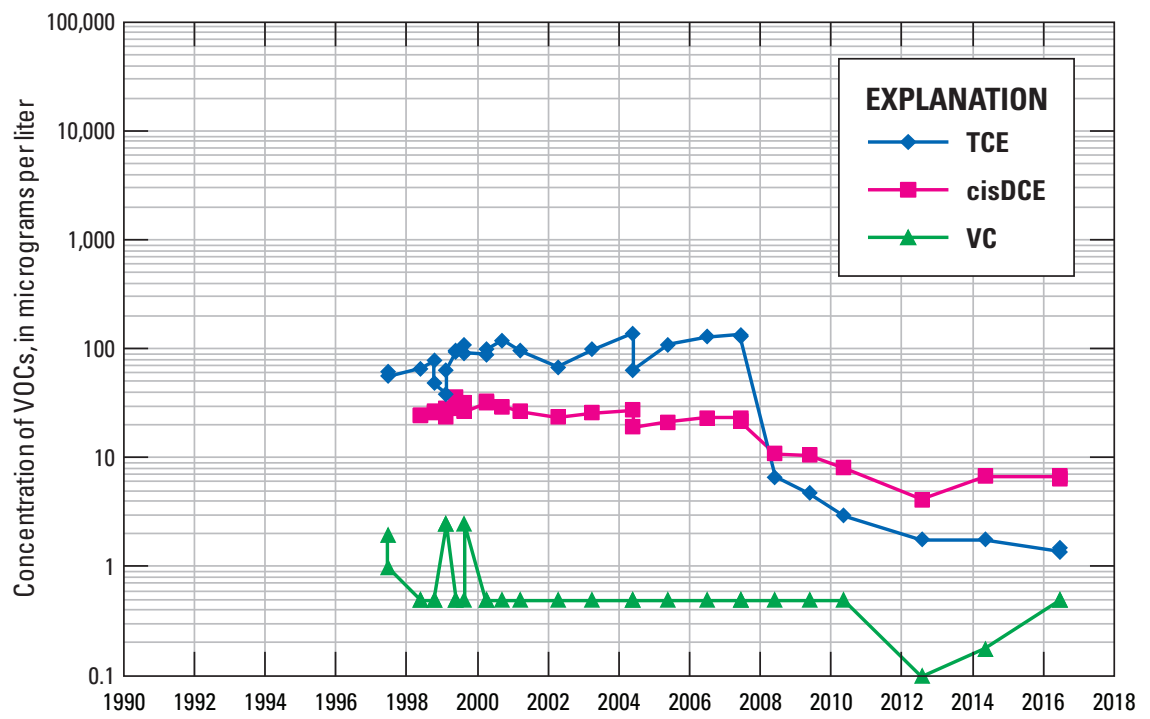

\section{Well 12BR}

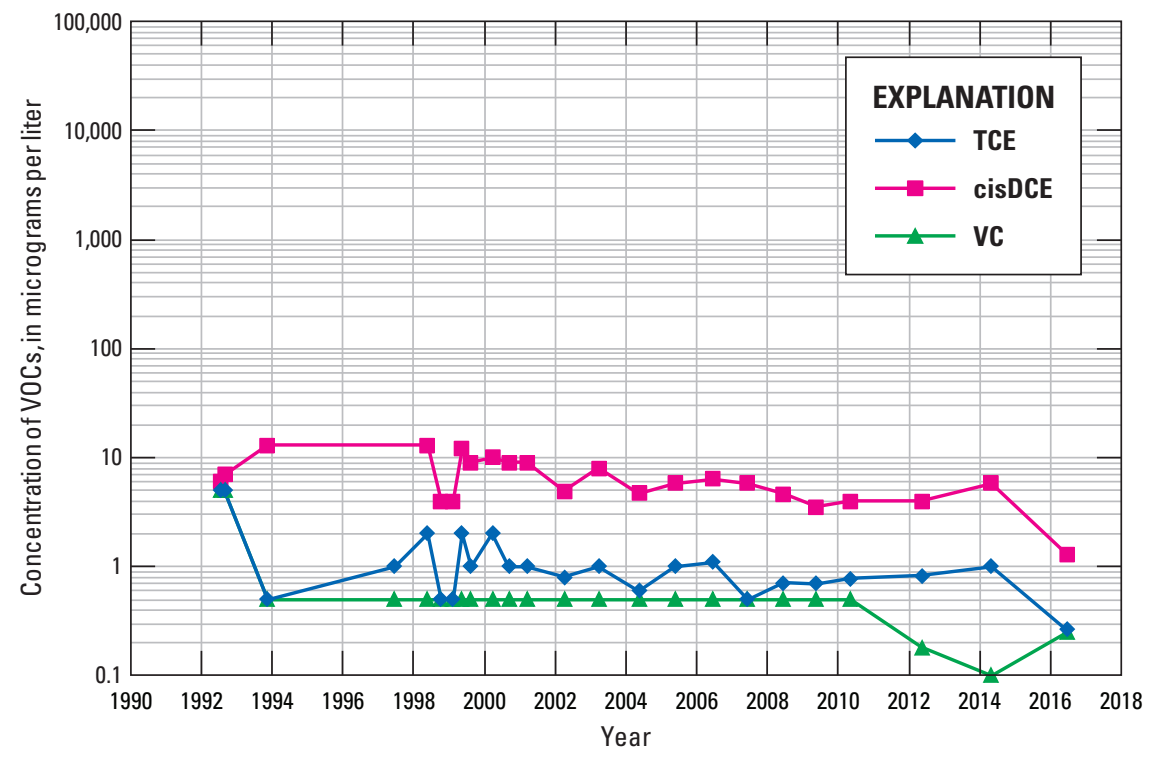

Well 11S

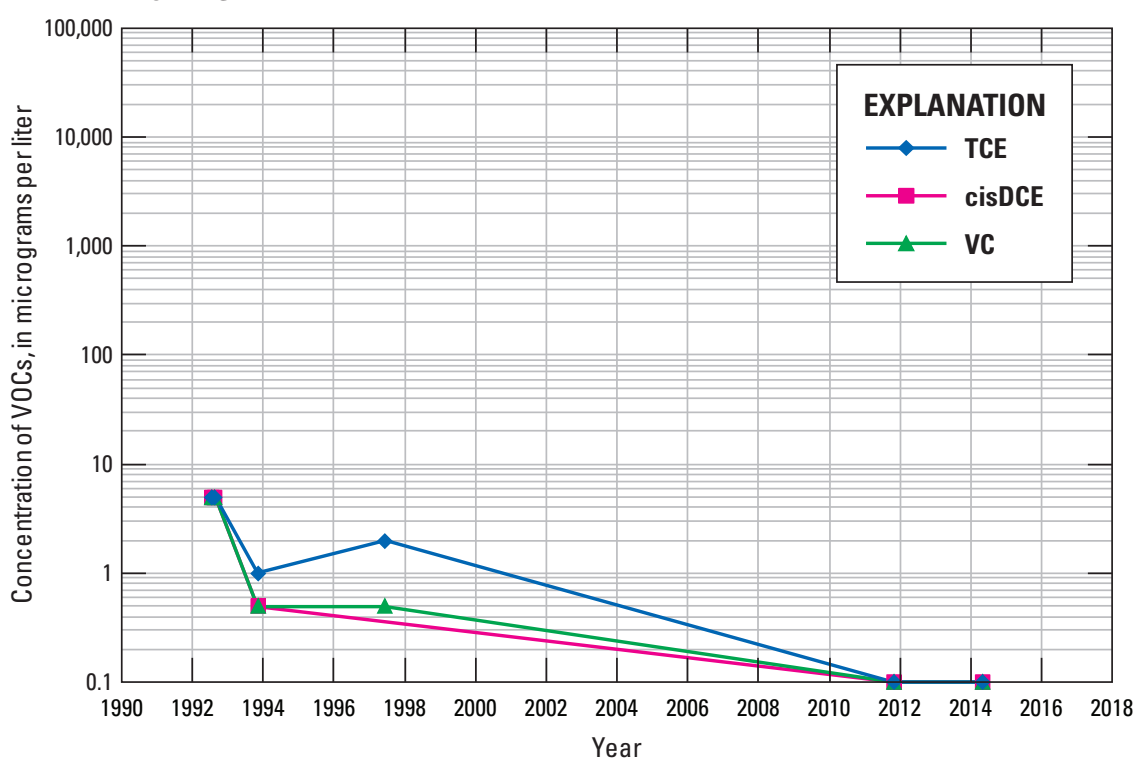

Figure 44. Volatile organic compound concentrations over time in wells along section B-B', former Naval Air Warfare Center, West Trenton, New Jersey, 1990-2017. (V0C, volatile organic compound; TCE, trichloroethene; cisDCE, cis-1,2-dichloroethene; VC, vinyl chloride)—Continued 
Well 37BR

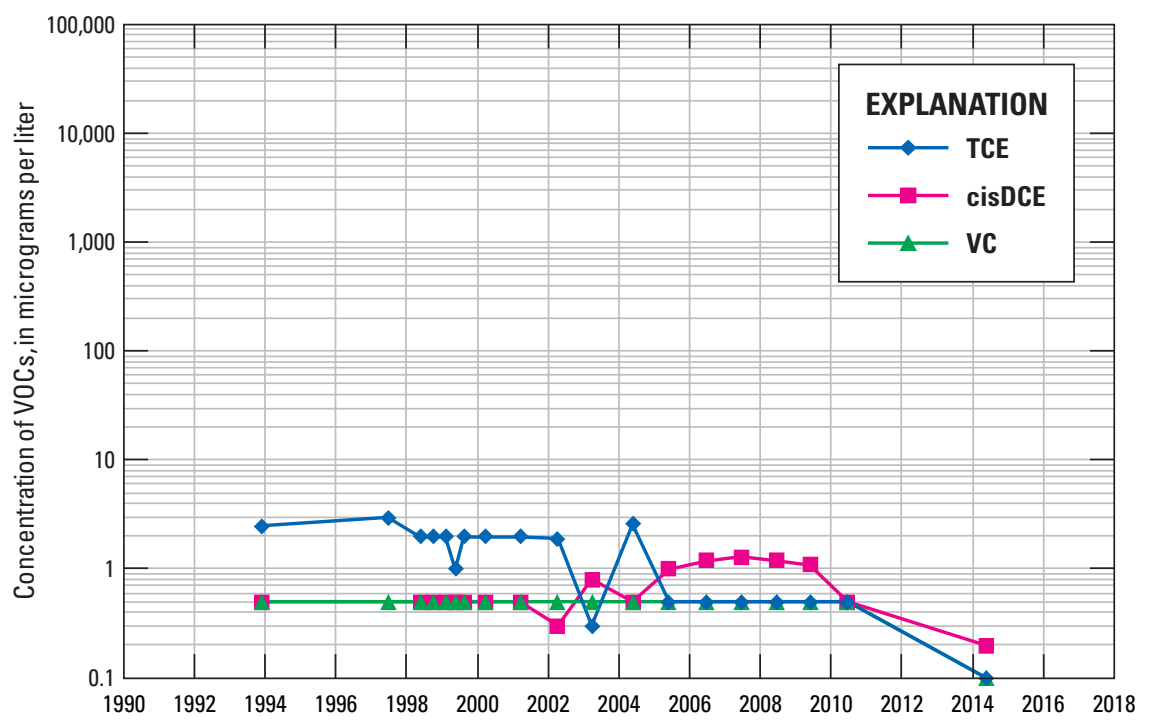

\section{Well 54BR}

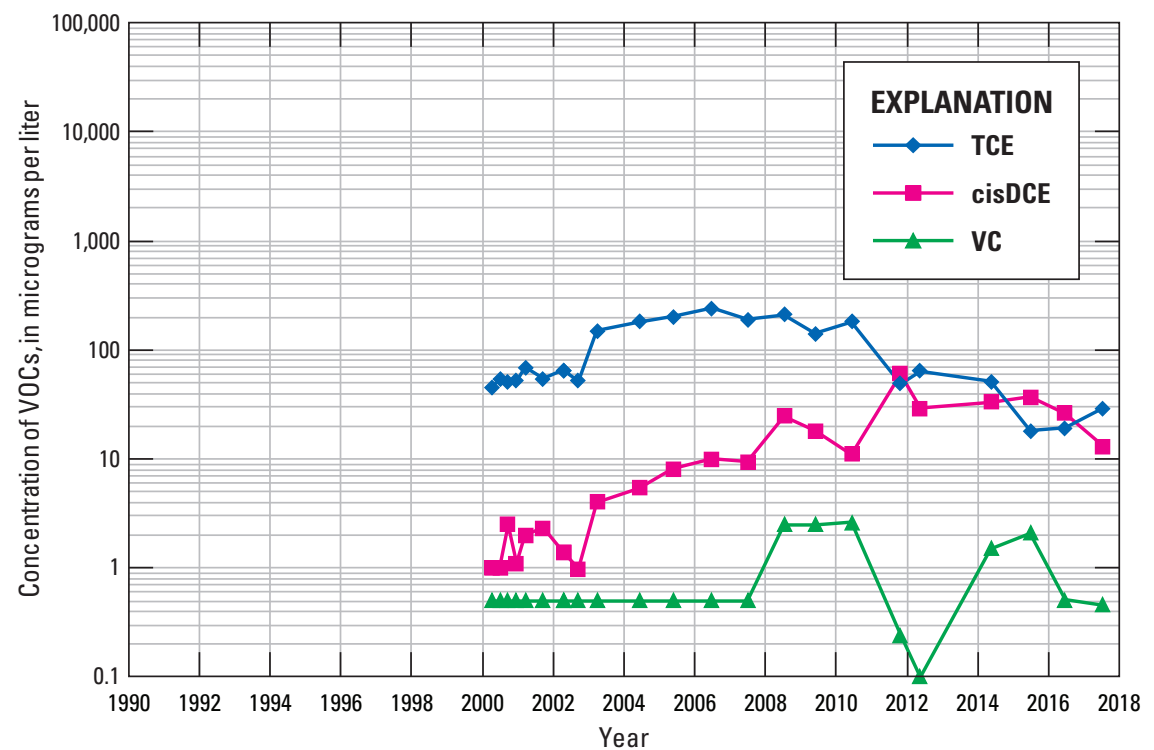

Well 11MW1

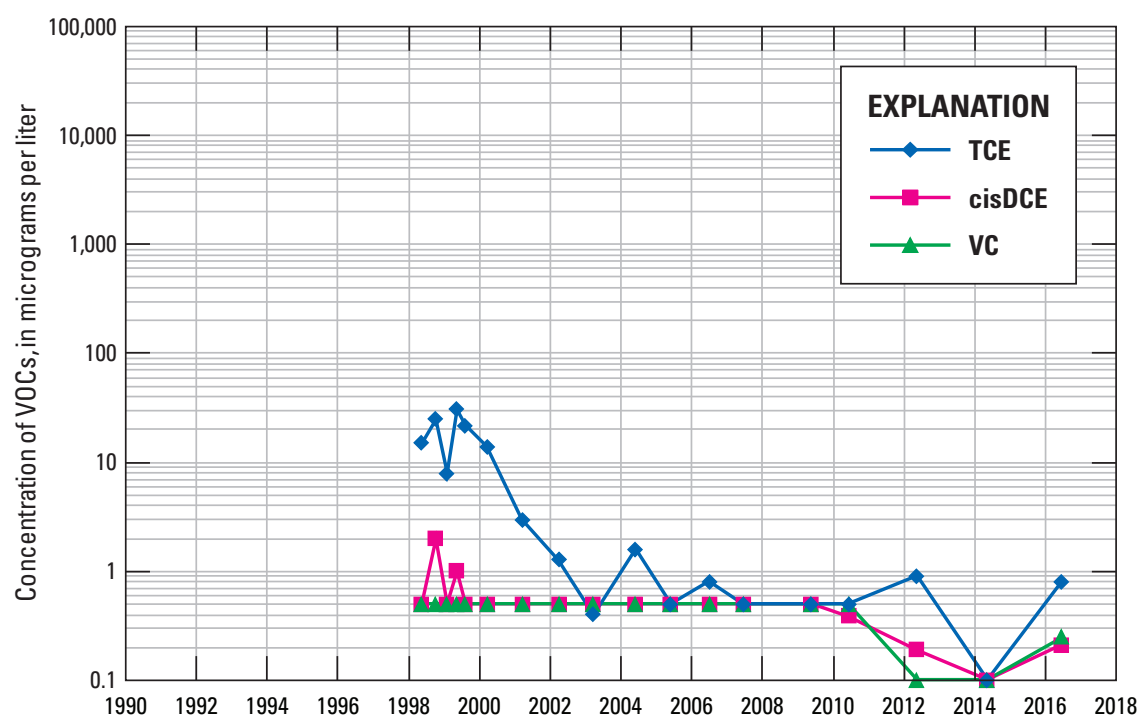

\section{Well 31BR}

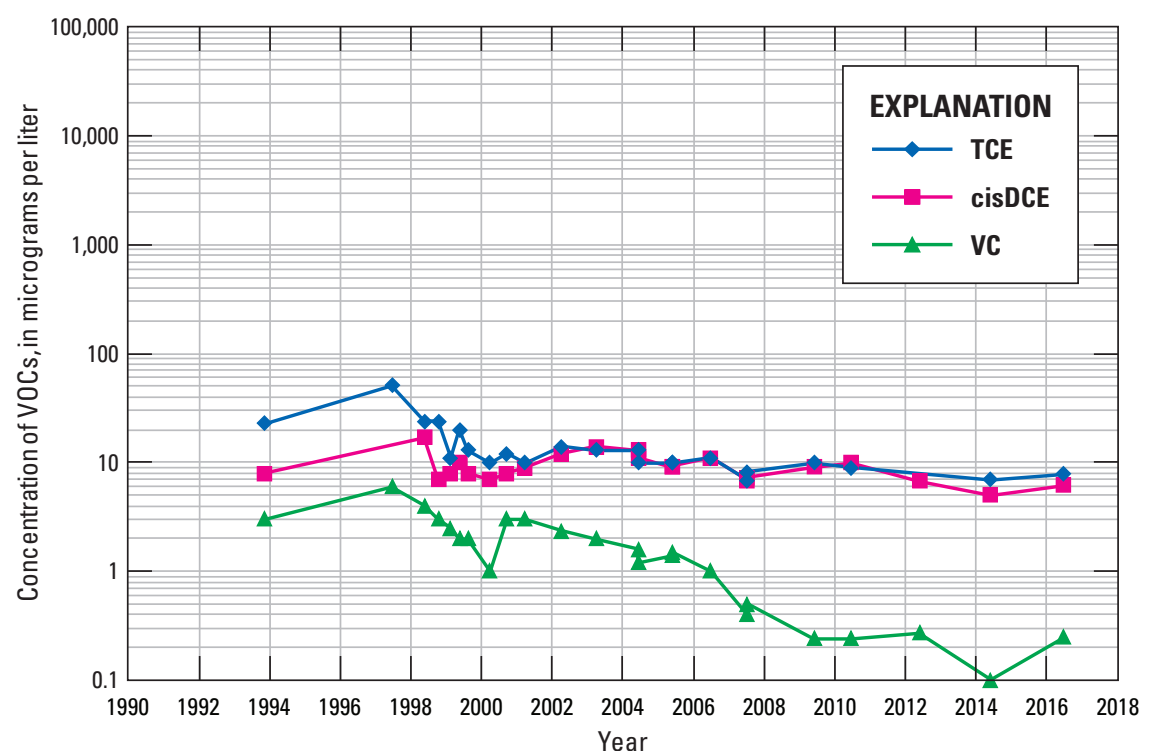

Figure 45. Volatile organic compound concentrations over time in wells along section C-C', former Naval Air Warfare Center, West Trenton, New Jersey 1992-2017. (VOC, volatile organic compound; TCE, trichloroethene; cisDCE, cis-1,2-dichloroethene; VC, vinyl chloride) 
Well $31 S$

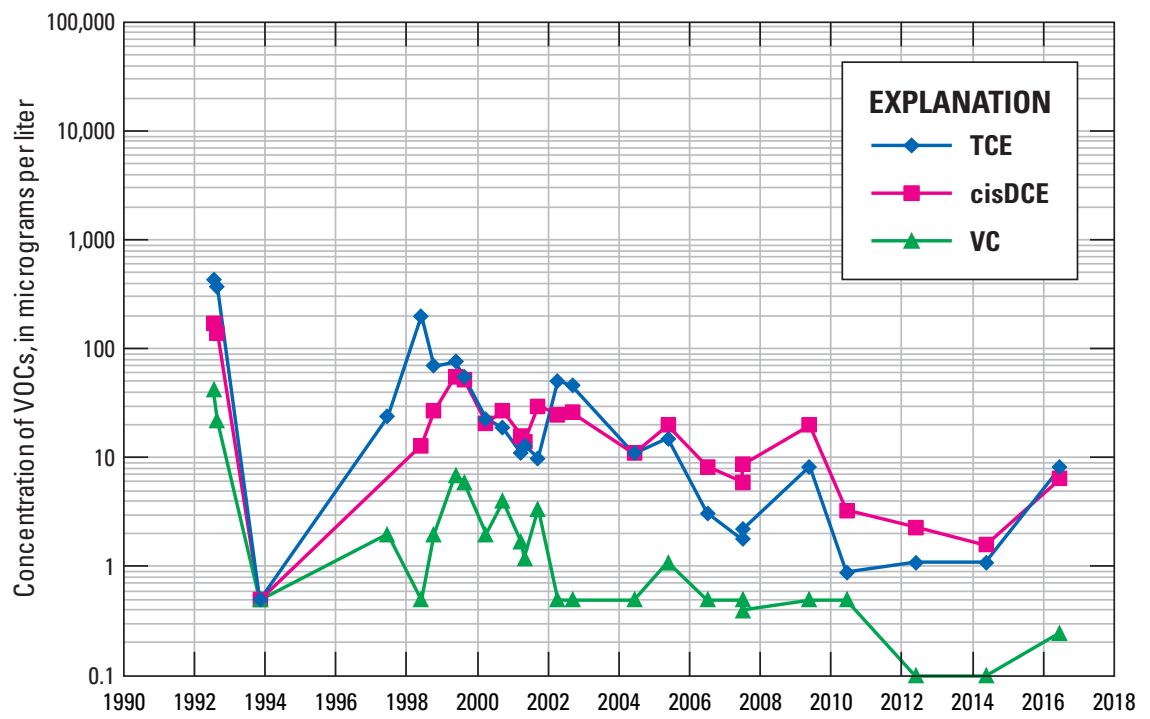

Well 48BR

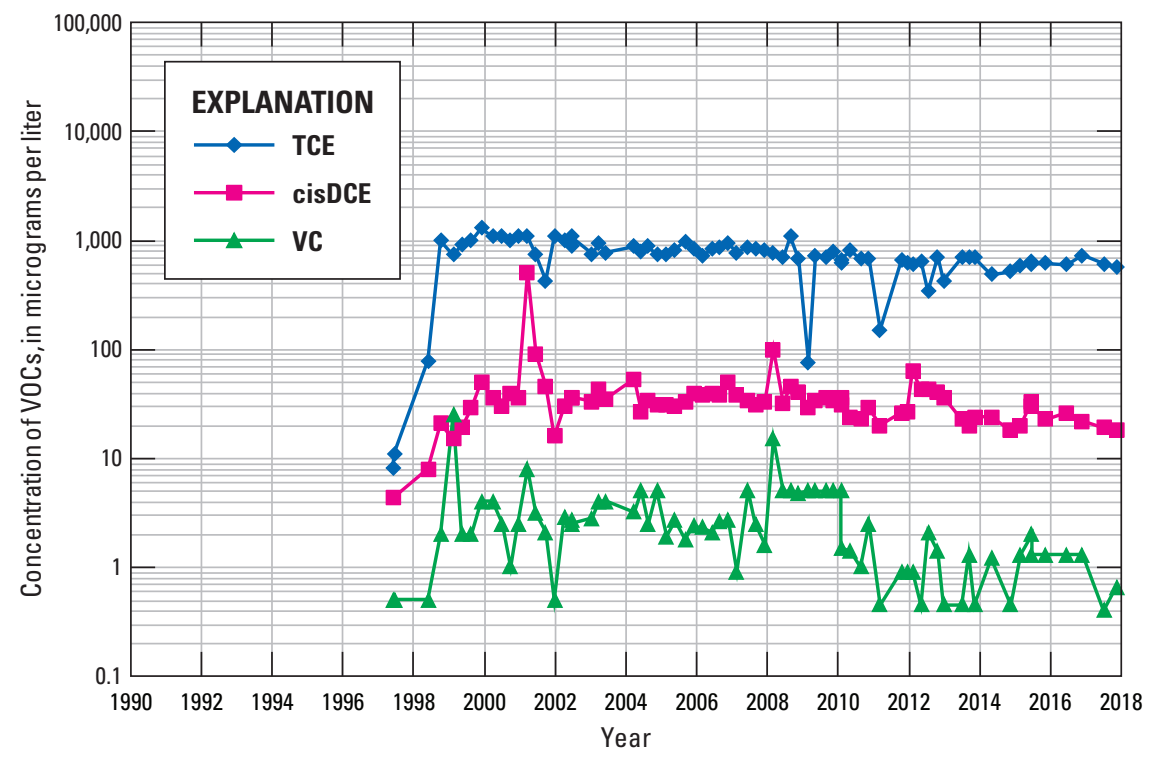

Well 30S

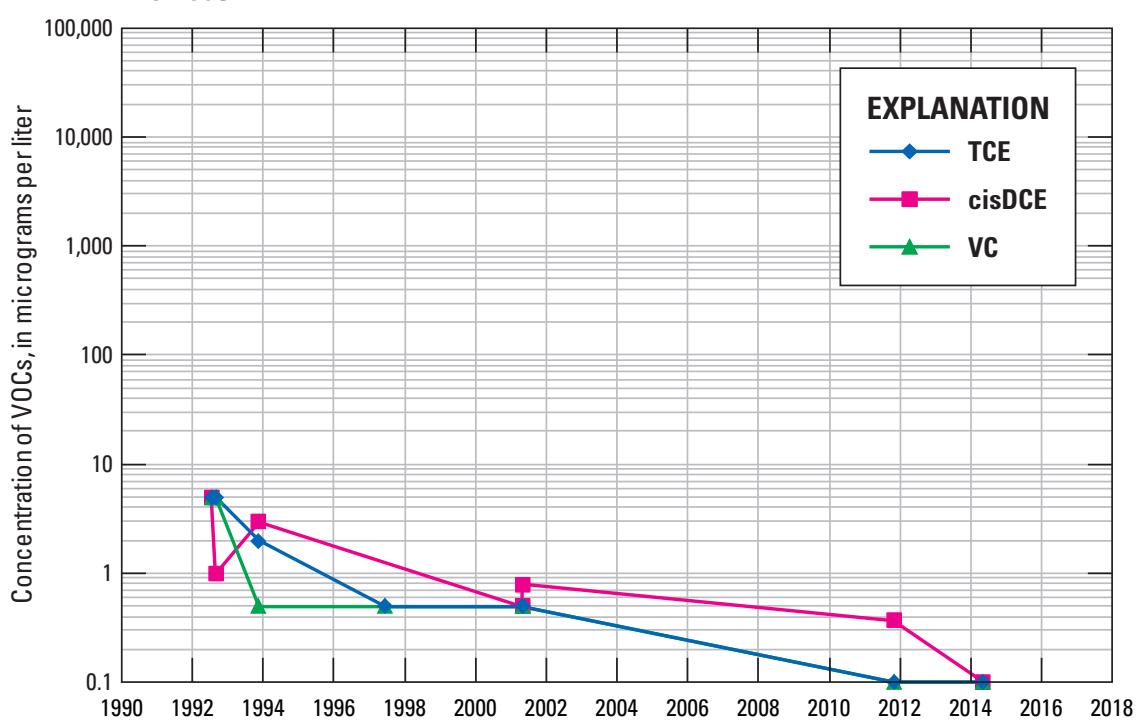

Well 14BR

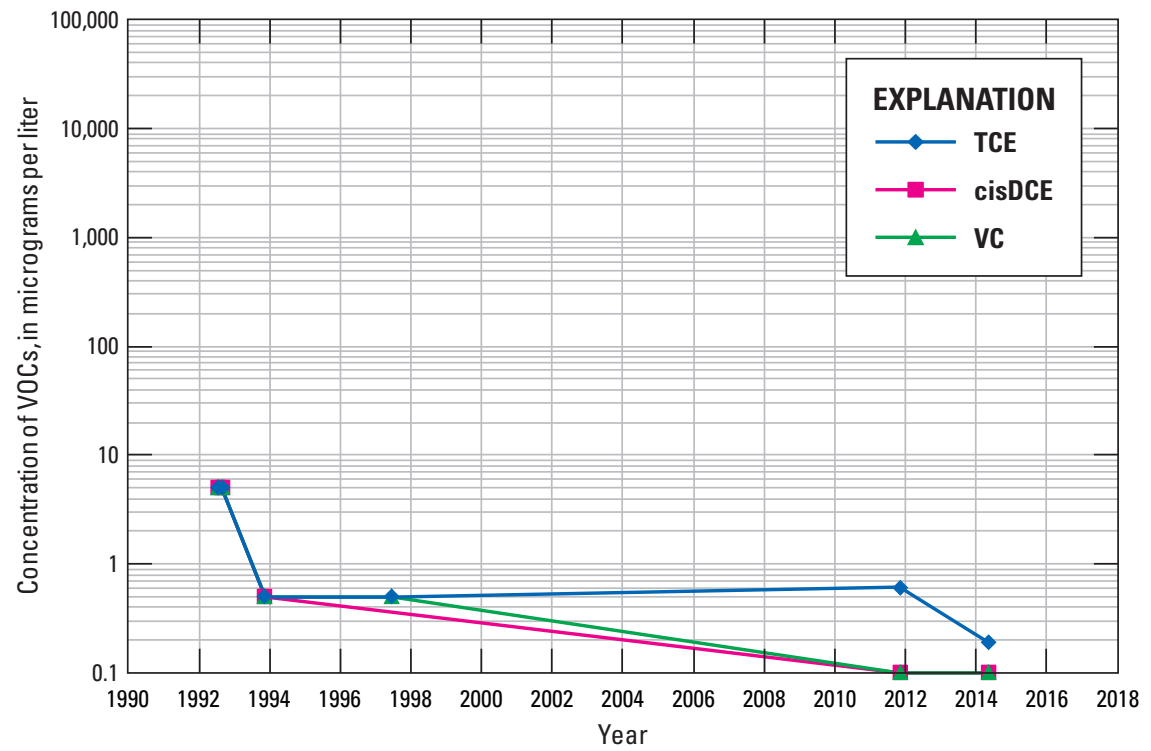

Figure 45. Volatile organic compound concentrations over time in wells along section C-C', former Naval Air Warfare Center, West Trenton, New Jersey 1992-2017. (V0C, volatile organic compound; TCE, trichloroethene; cisDCE, cis-1,2-dichloroethene; VC, vinyl chloride)—Continued 
Well 34S

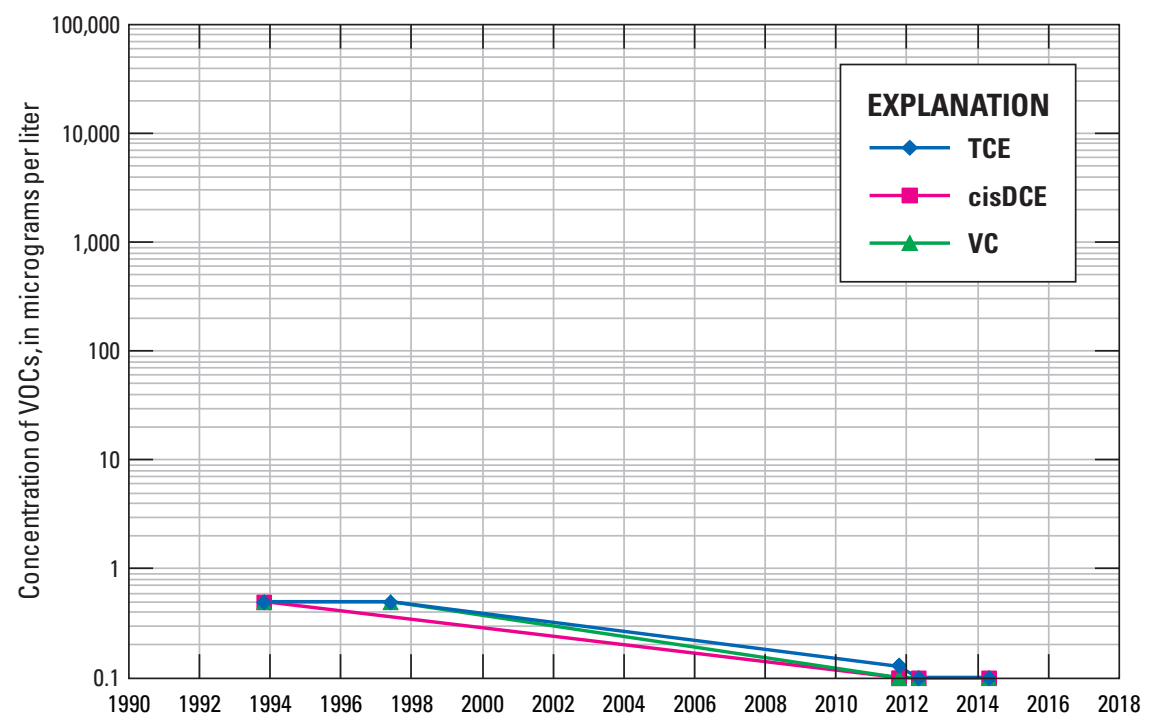

\section{Well 49BR}

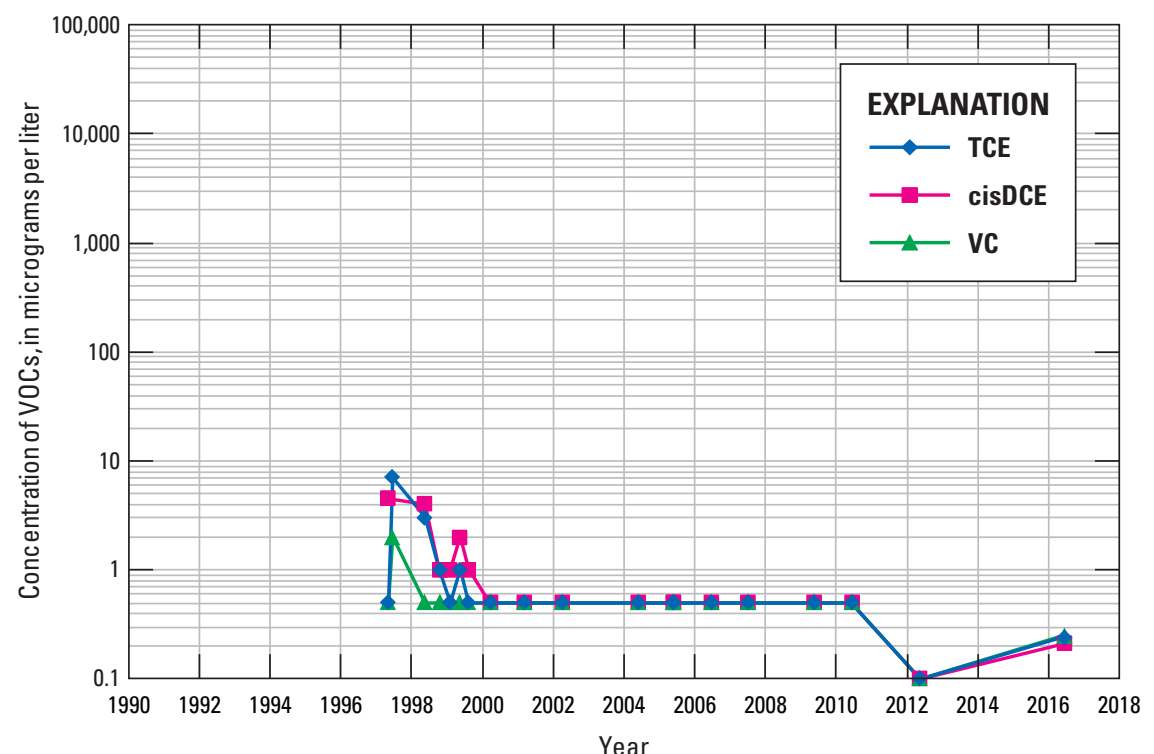

Well 37S

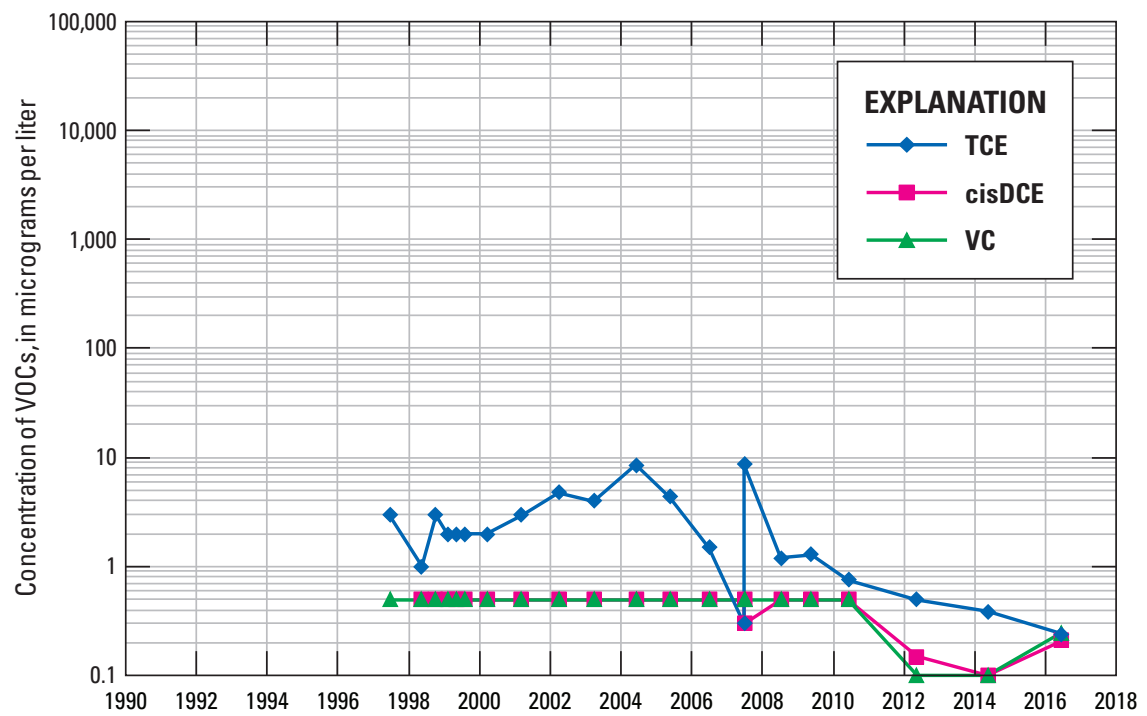

Well 53BR

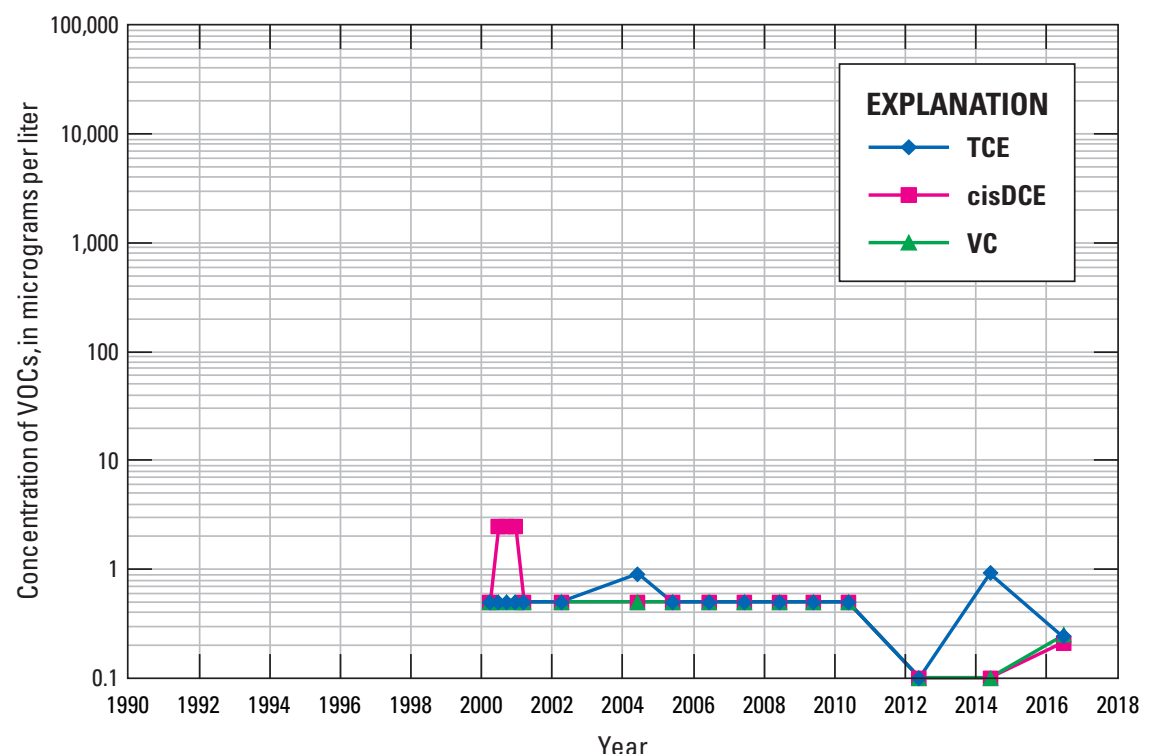

Figure 46. Volatile organic compound concentrations over time in wells along section D-D', former Naval Air Warfare Center, West Trenton, New Jersey, 1992-2017. (V0C, volatile organic compound; TCE, trichloroethene; cisDCE, cis-1,2-dichloroethene; VC, vinyl chloride) 


\section{Well 44BR}

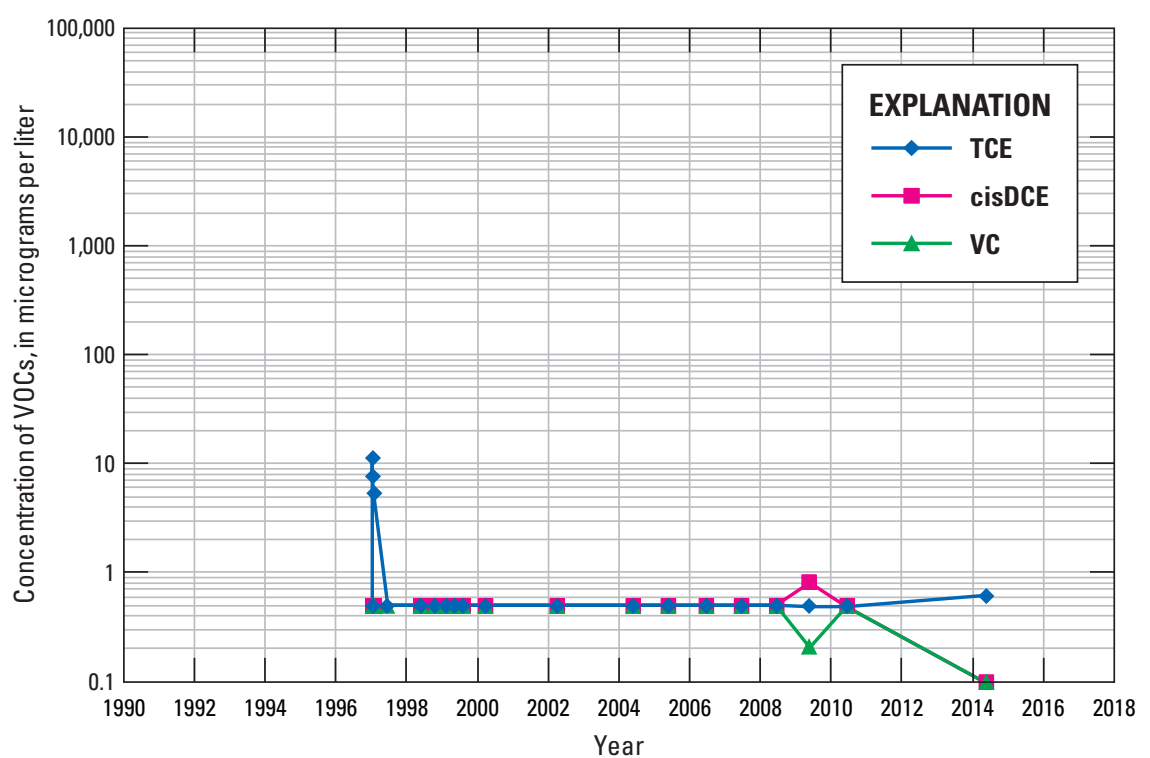

Well 43BR

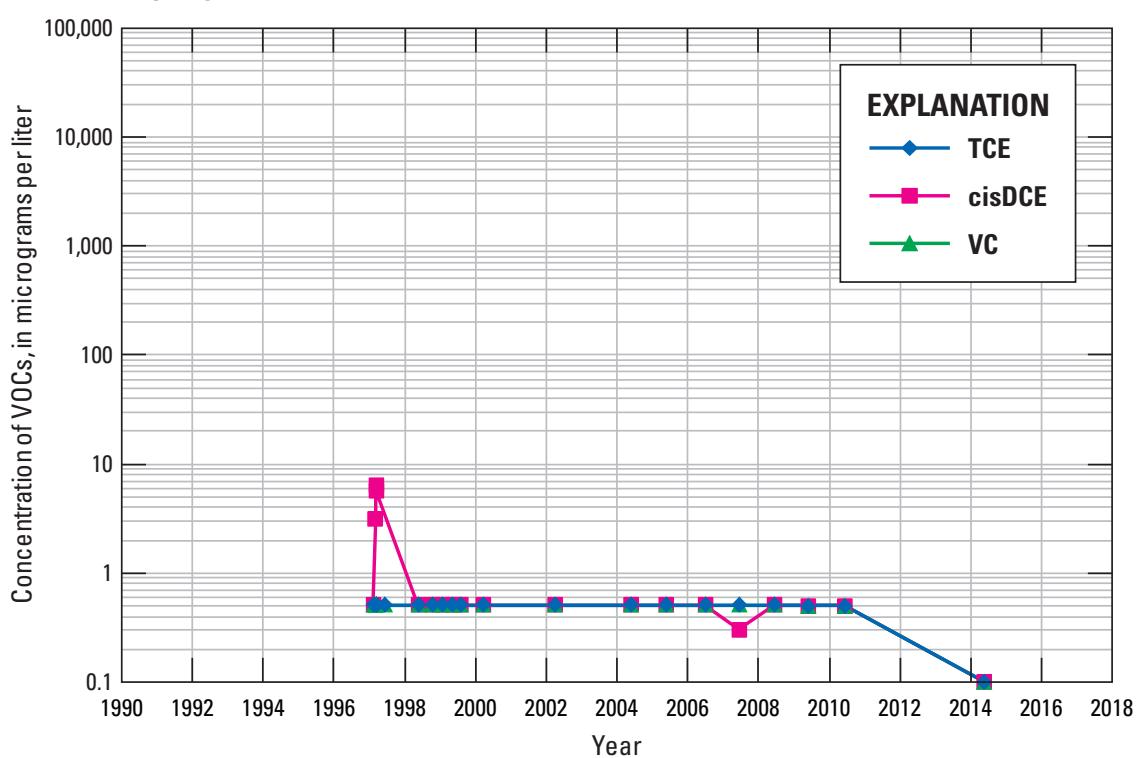

Figure 46. Volatile organic compound concentrations over time in wells along section D-D', former Naval Air Warfare Center, West Trenton, New Jersey, 1992-2017. (VOC, volatile organic compound; TCE, trichloroethene; cisDCE, cis-1,2-dichloroethene; VC, vinyl chloride)—Continued 
Well 06BR

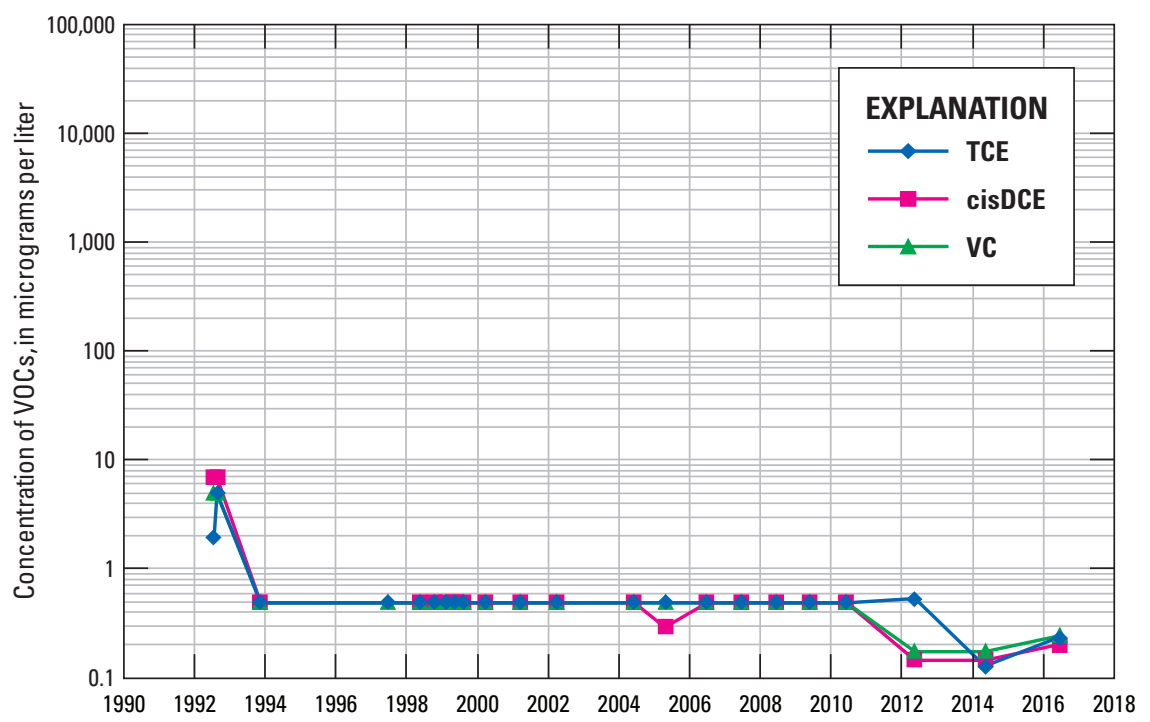

\section{Well 39BR}

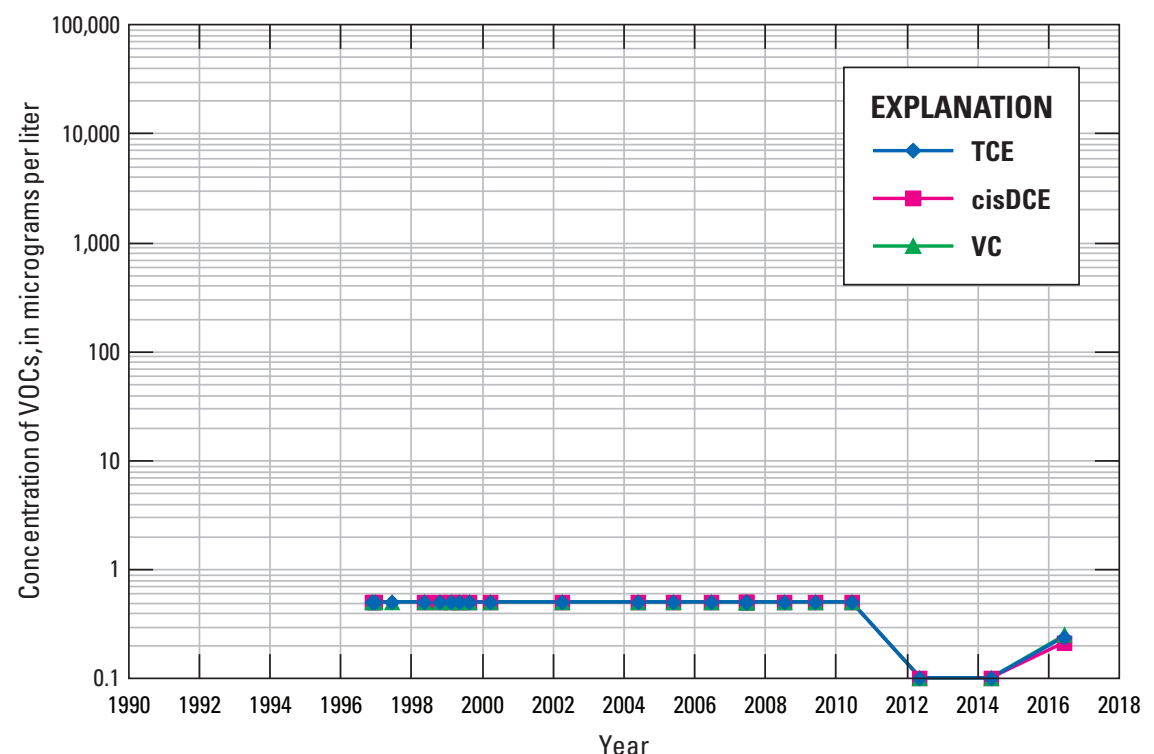

Well 42BR

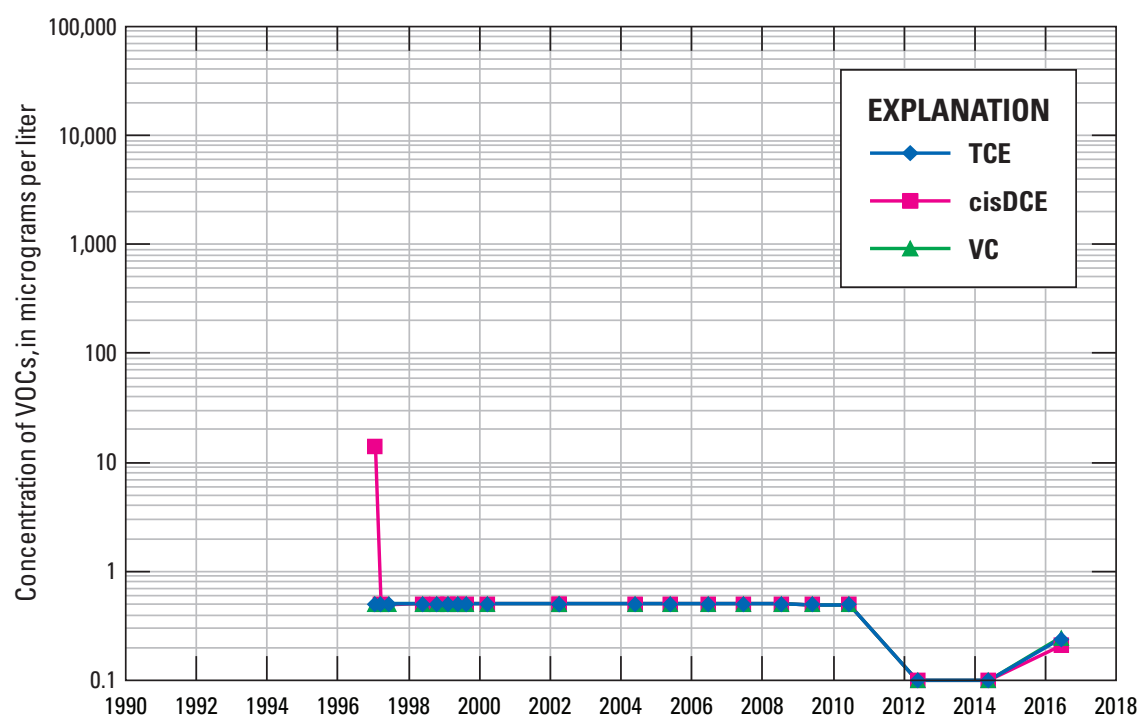

\section{Well 16BR}

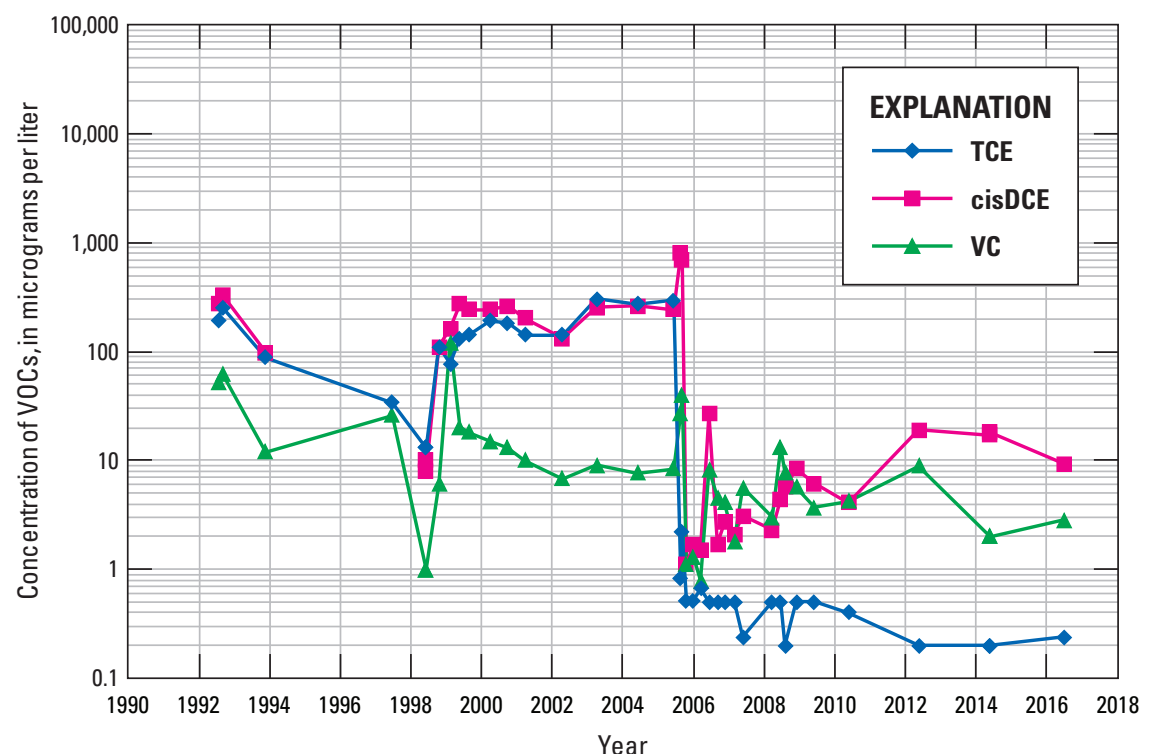

Figure 47. Volatile organic compound concentrations over time in wells along section E-E', former Naval Air Warfare Center, West Trenton, New Jersey, 1992-2017. (VOC, volatile organic compound; TCE, trichloroethene; cisDCE, cis-1,2-dichloroethene; VC, vinyl chloride) 


\section{Well 41BR}

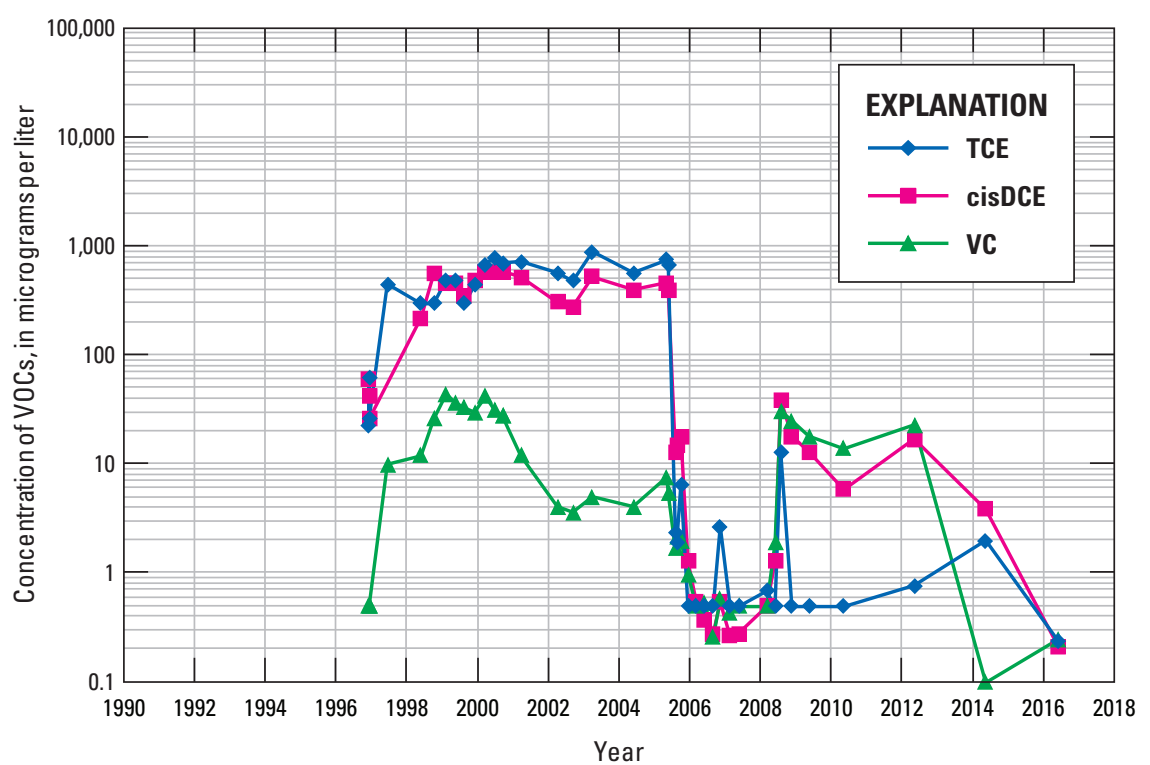

\section{Well 16S}

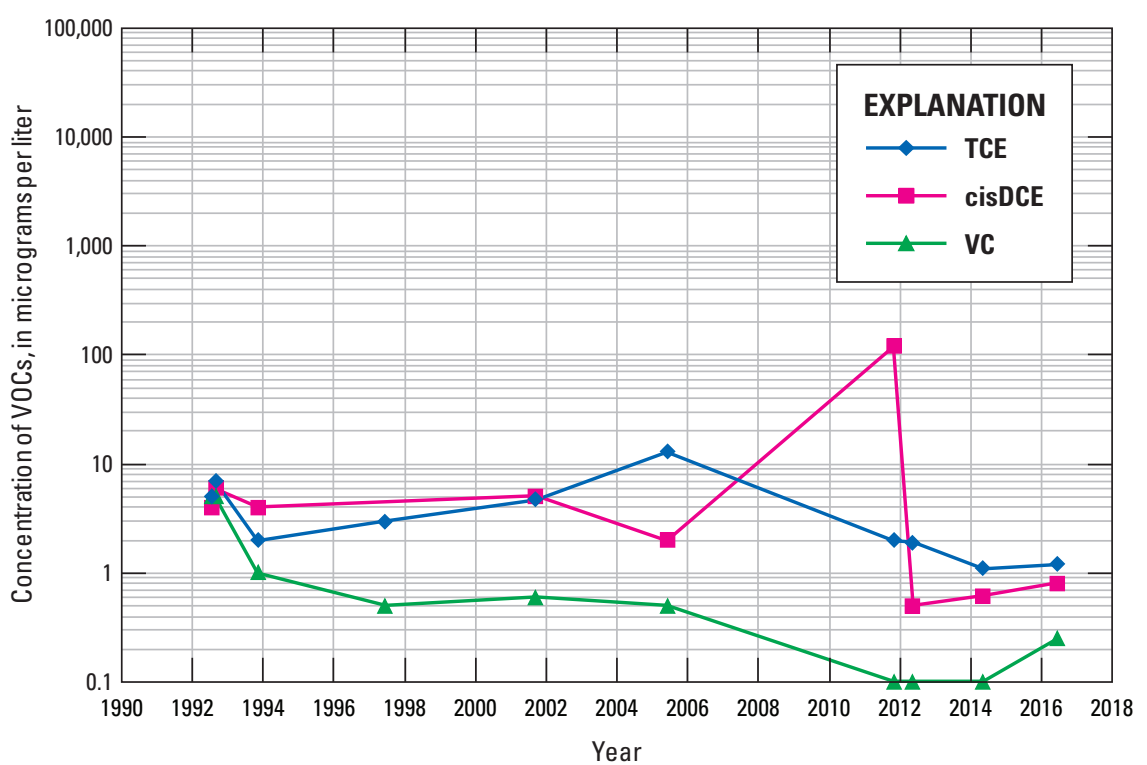

Figure 47. Volatile organic compound concentrations over time in wells along section E-E', former Naval Air Warfare Center, West Trenton, New Jersey, 1992-2017. (VOC, volatile organic compound; TCE, trichloroethene; cisDCE, cis-1,2-dichloroethene; VC, vinyl chloride)—Continued 
Well 28BR

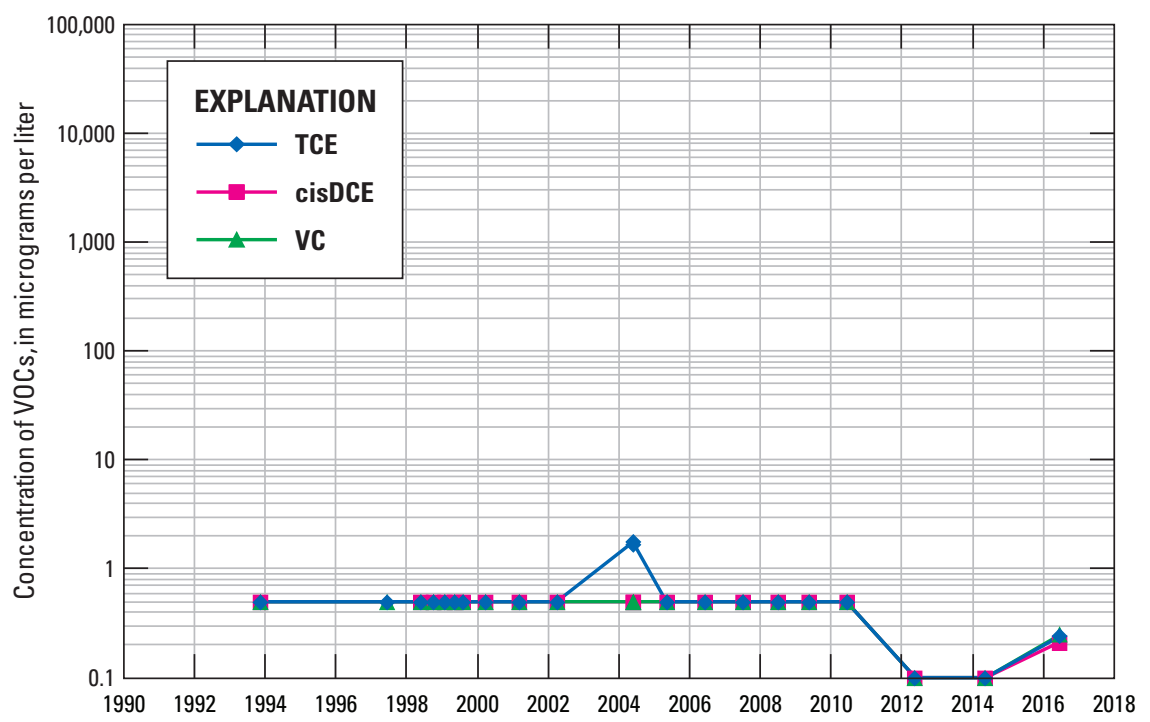

Well 22BR

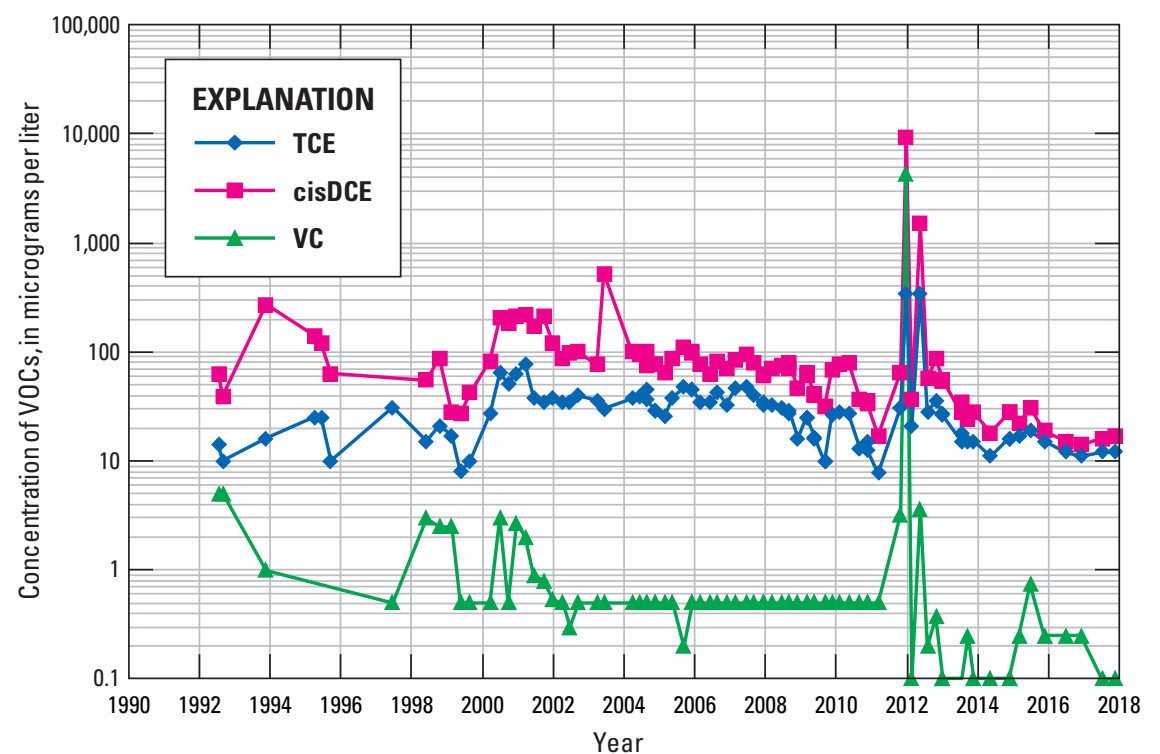

Well 52BR

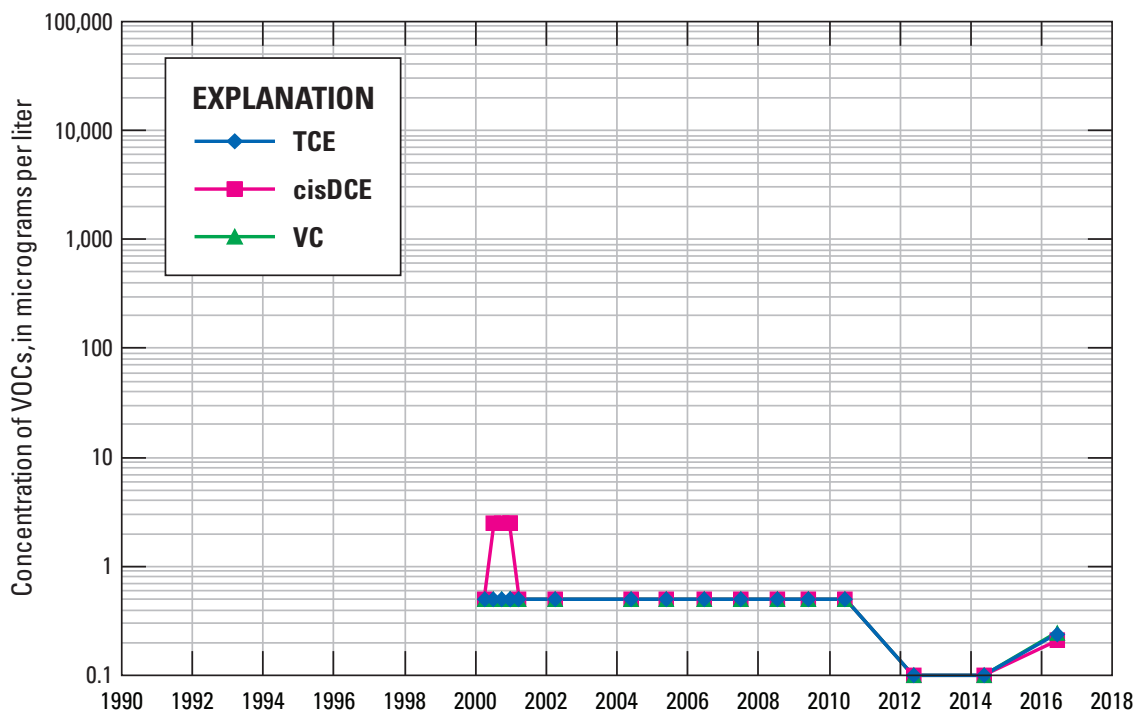

\section{Well 23BR}

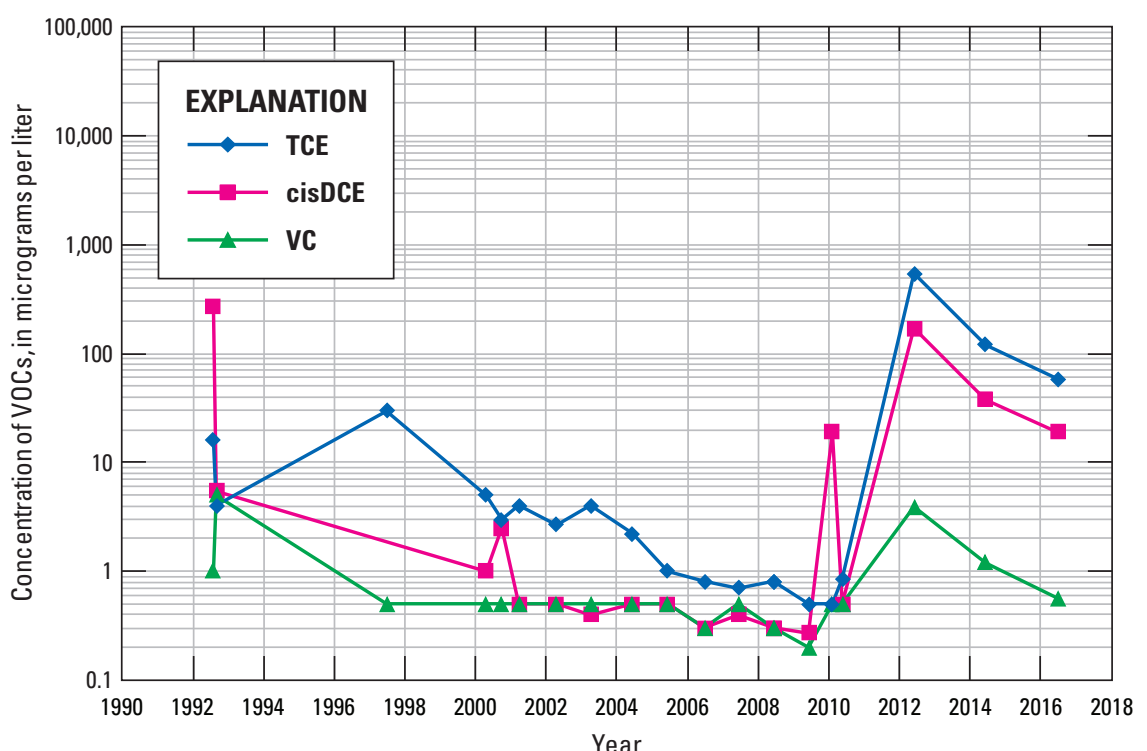

Figure 48. Volatile organic compound concentrations over time in wells along section F-F', former Naval Air Warfare Center, West Trenton, New Jersey, 1992-2017. (VOC, volatile organic compound; TCE, trichloroethene; cisDCE, cis-1,2-dichloroethene; VC, vinyl chloride) 
Well 04BR

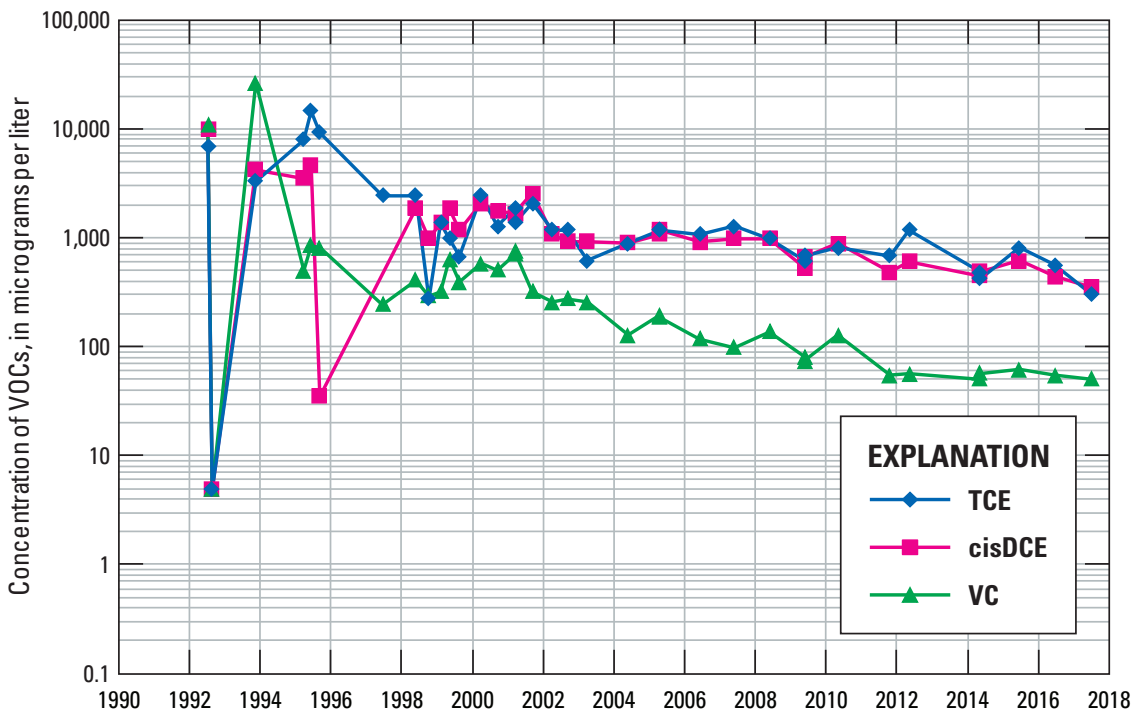

\section{Well 30BR}

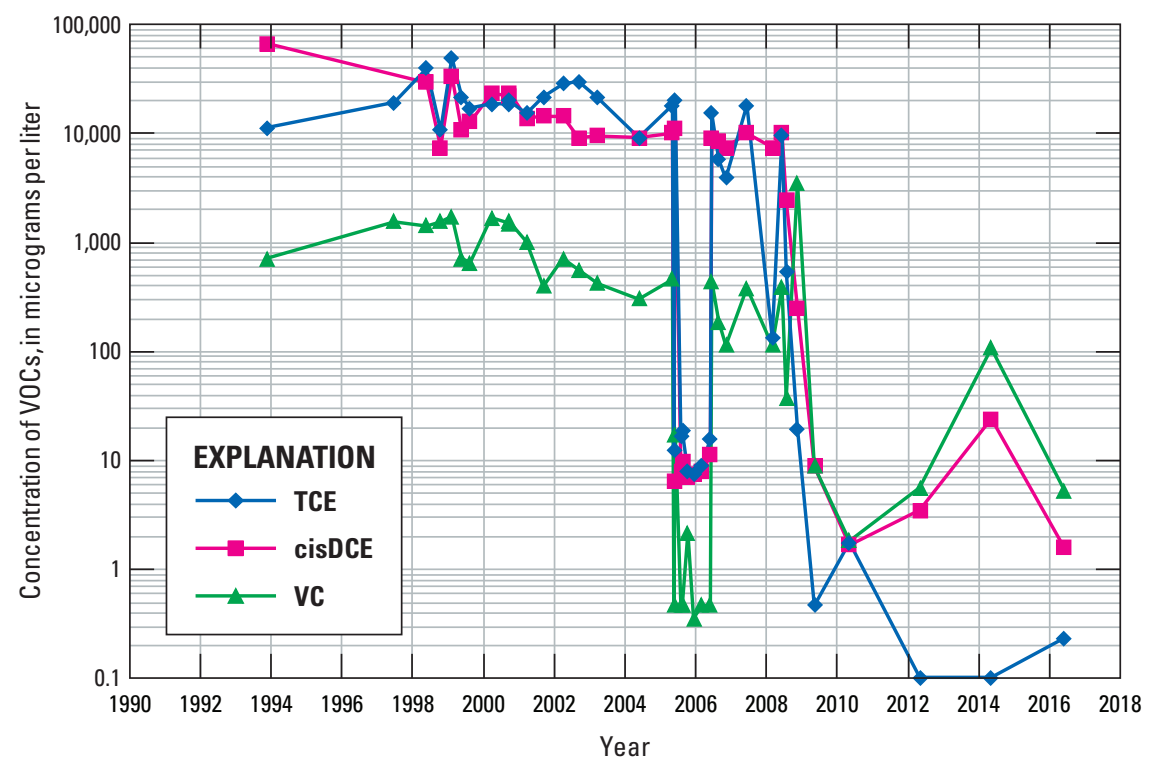

Well 17BR

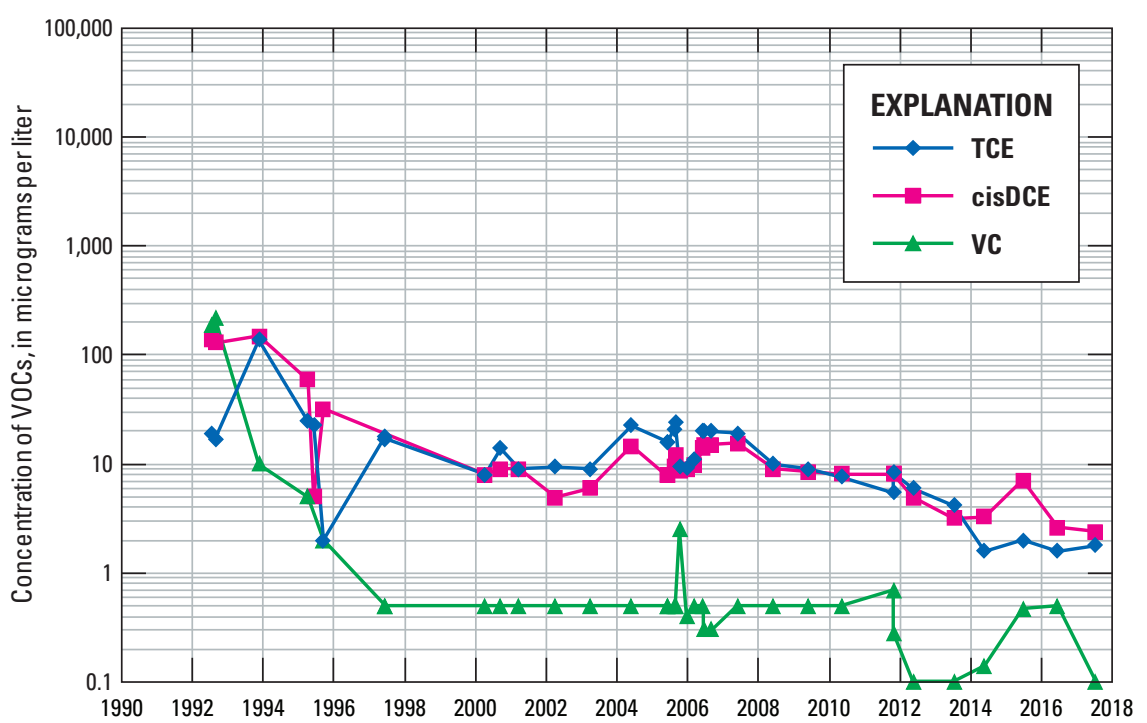

Well 47BR

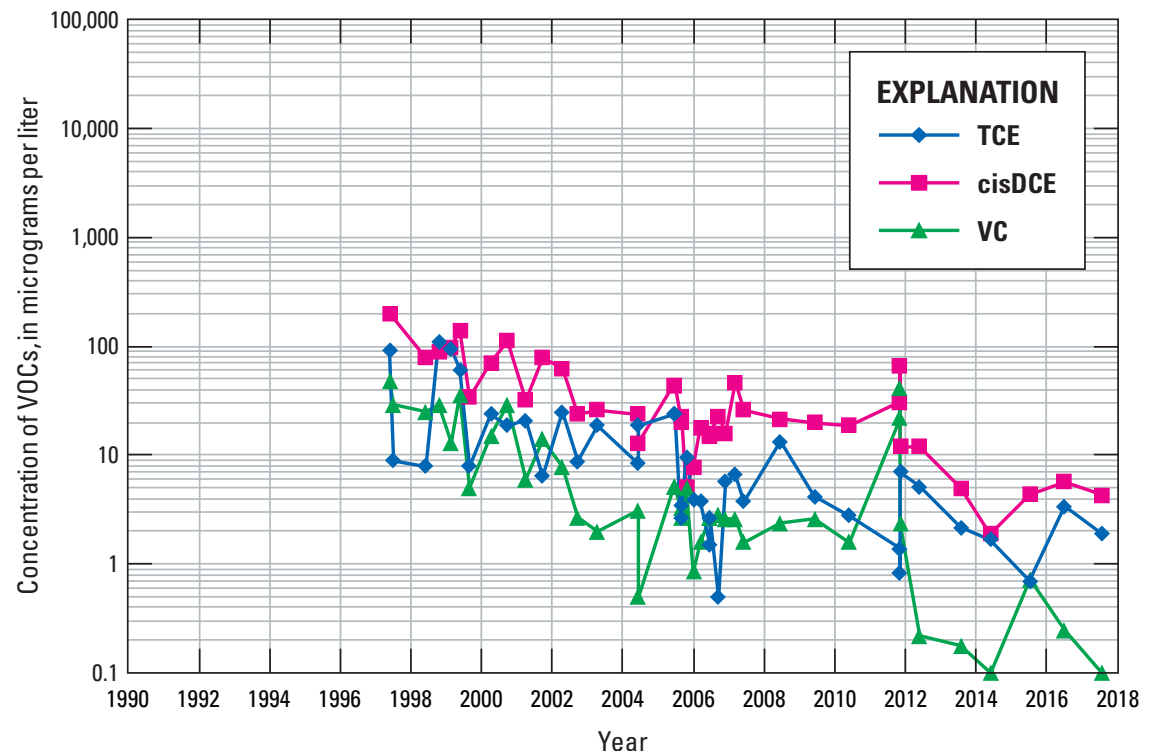

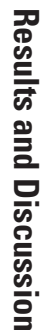

Figure 48. Volatile organic compound concentrations over time in wells along section F-F', former Naval Air Warfare Center, West Trenton, New Jersey, 1992-2017. (VOC, volatile organic compound; TCE, trichloroethene; cisDCE, cis-1,2-dichloroethene; VC, vinyl chloride)—Continued 
Well BRP01

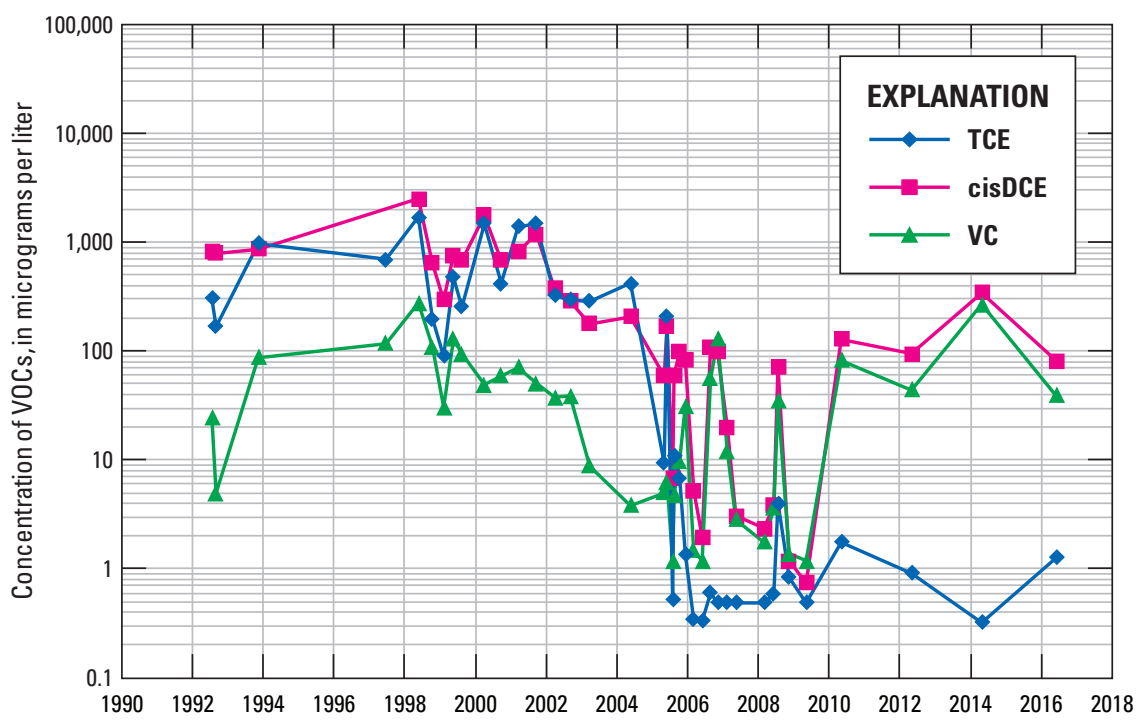

\section{Well 38BR}

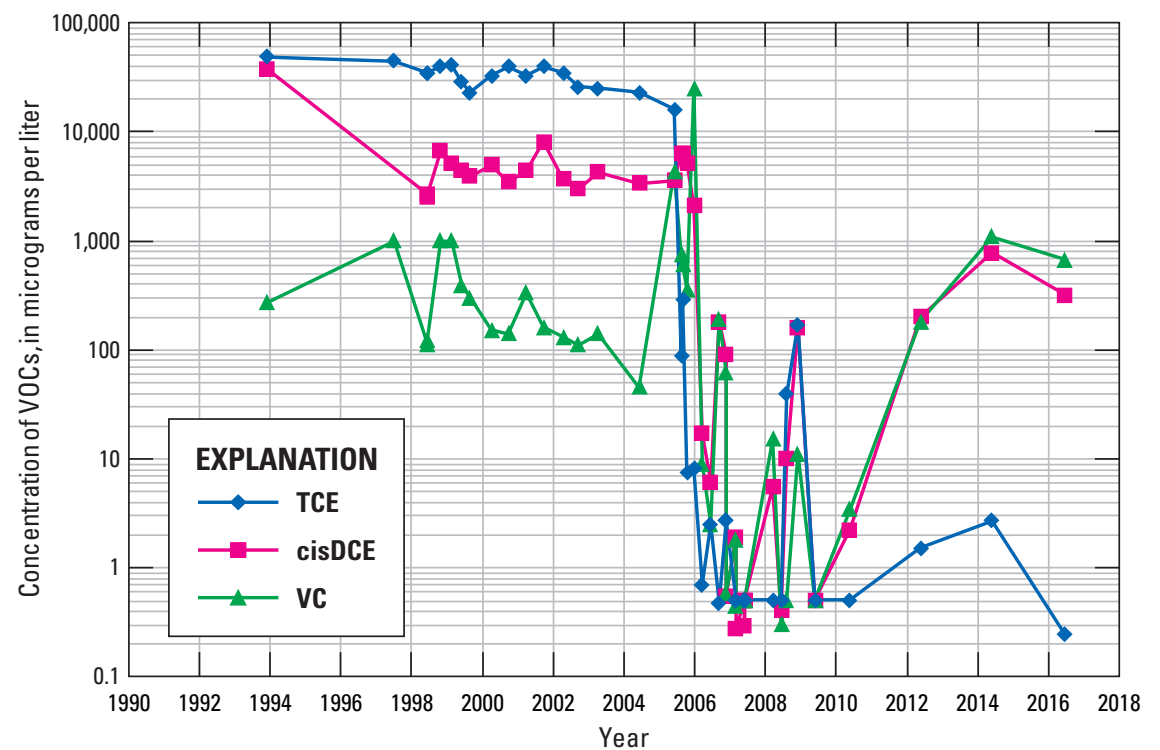

Well 74BR

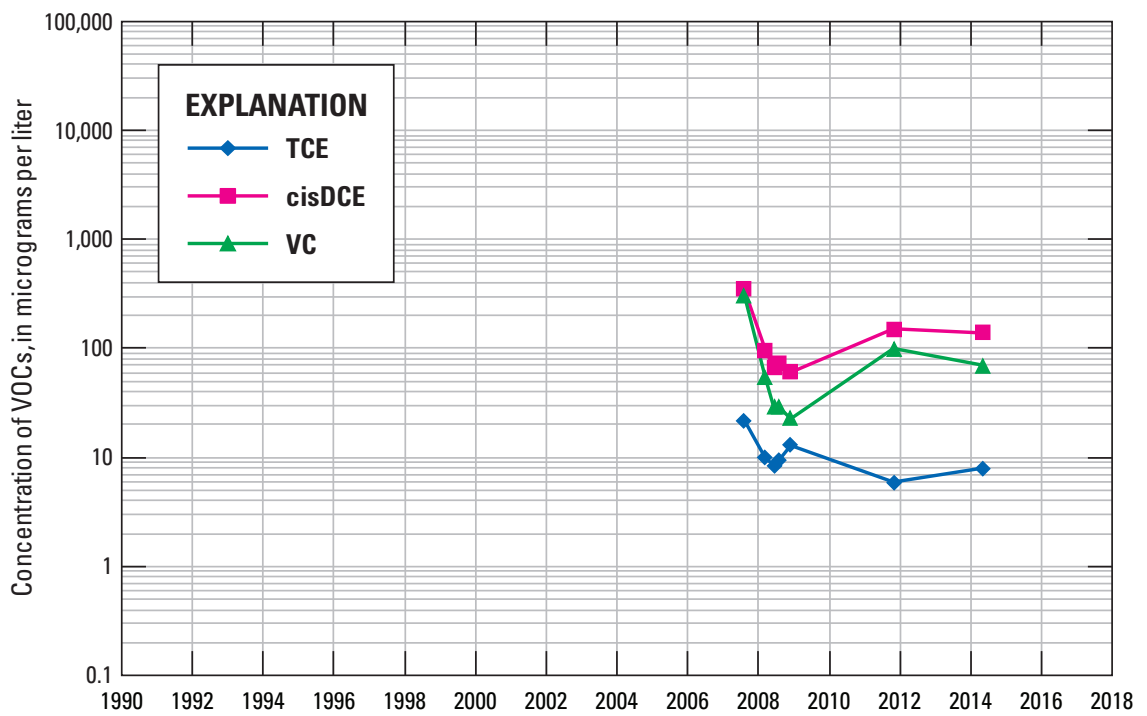

\section{Well 46BR}

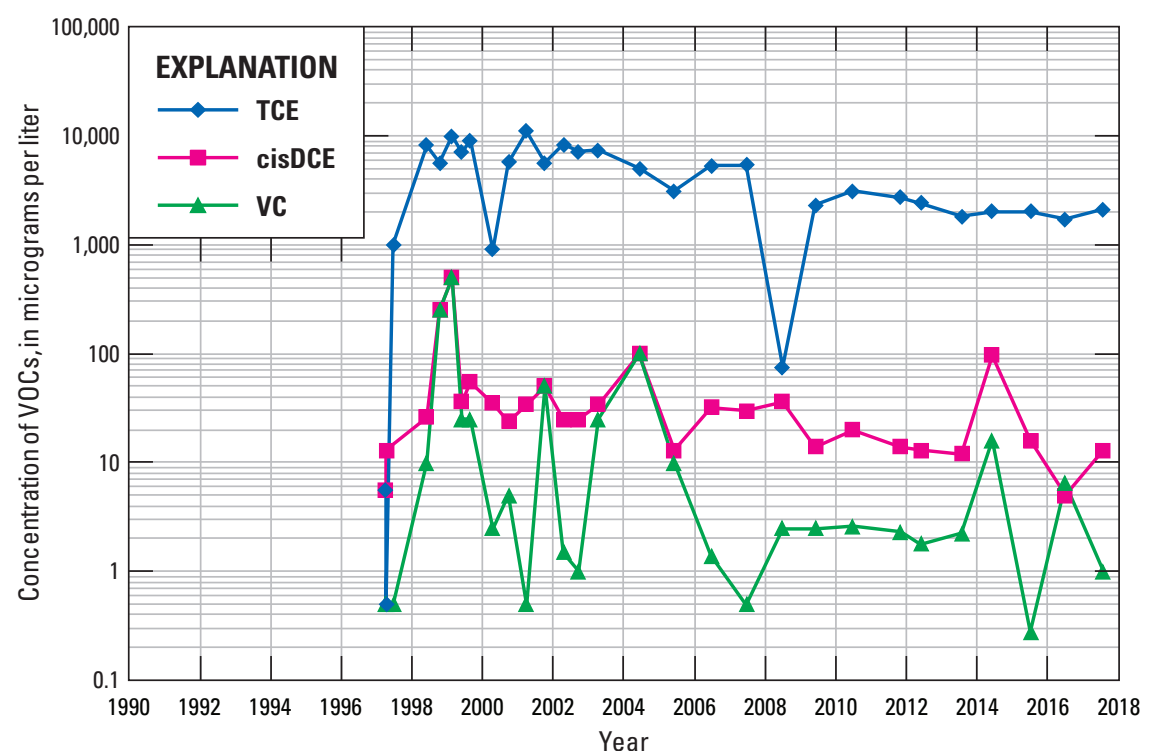

Figure 48. Volatile organic compound concentrations over time in wells along section F-F', former Naval Air Warfare Center, West Trenton, New Jersey, 1992-2017. (VOC, volatile organic compound; TCE, trichloroethene; cisDCE, cis-1,2-dichloroethene; VC, vinyl chloride)—Continued 


\section{Well 27BR}

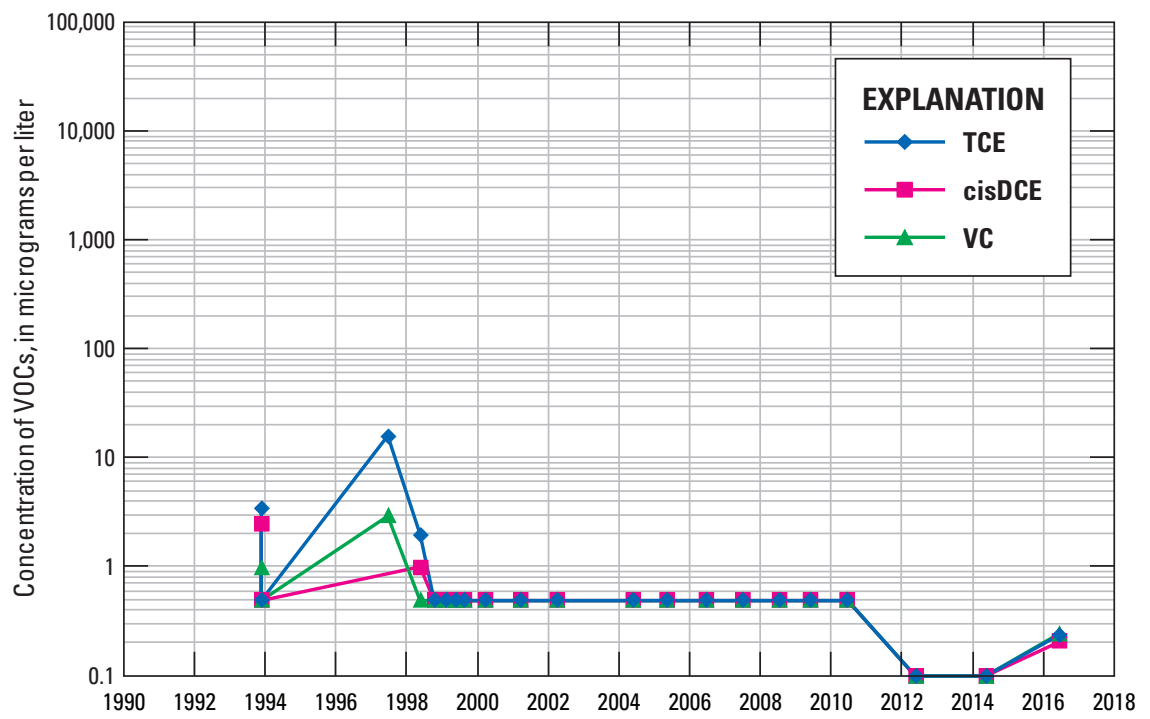

\section{Well 65BR}

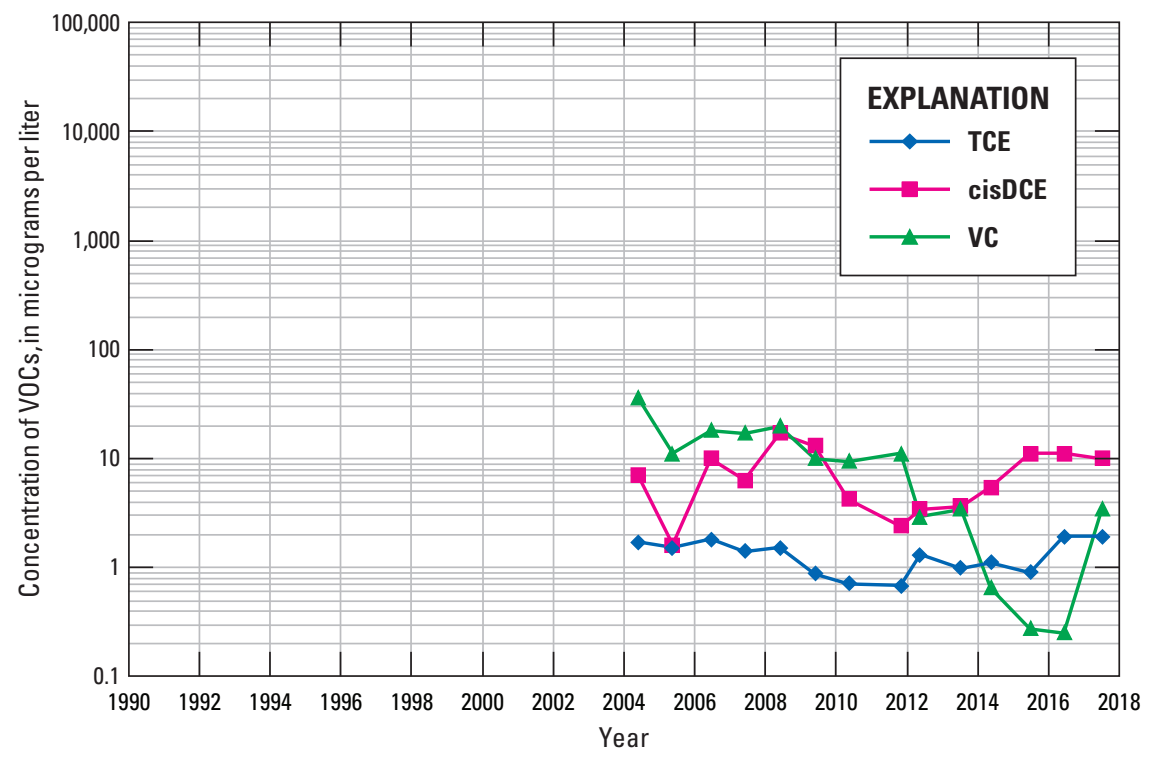

\section{Well 63BR}

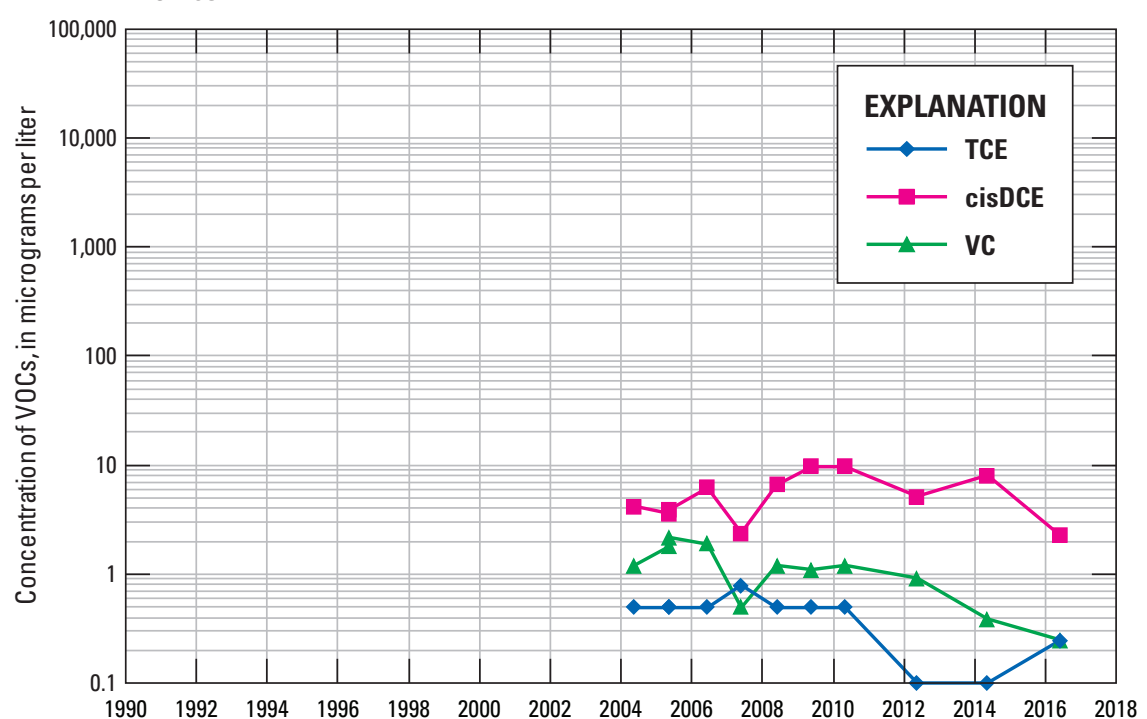

Well 24BR

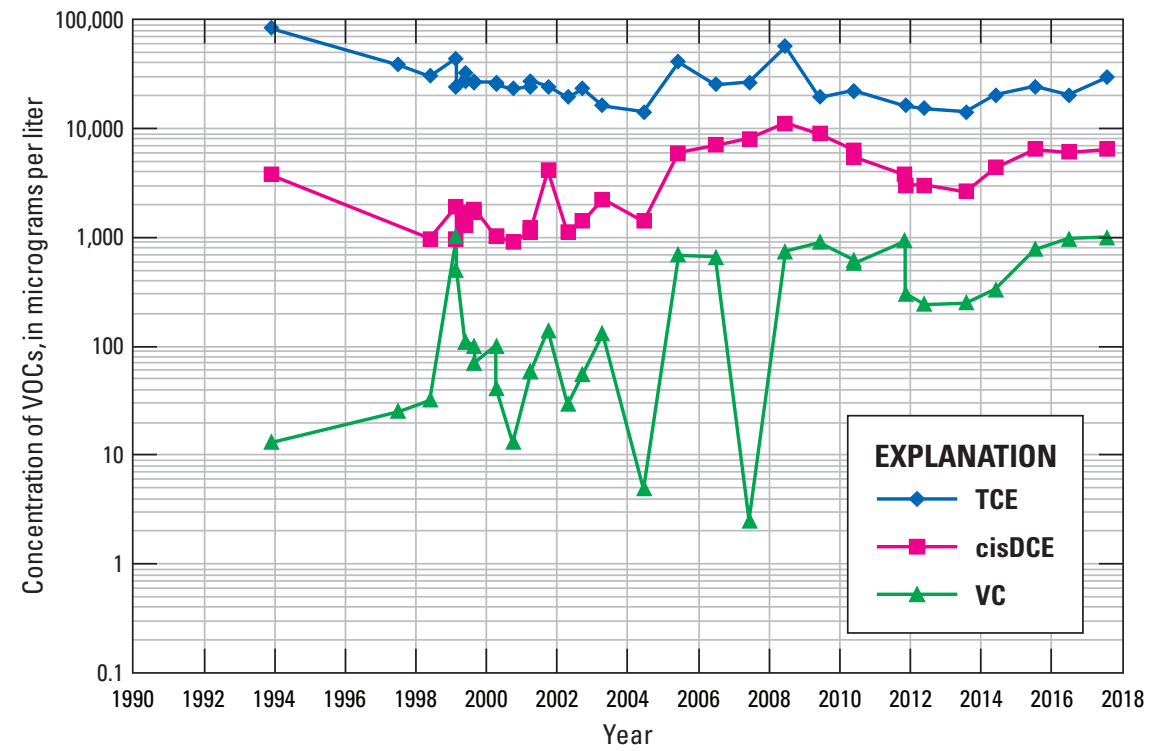

Figure 49. Volatile organic compound concentrations over time in wells along section G-G', former Naval Air Warfare Center, West Trenton, New Jersey, 1992-2017. (VOC, volatile organic compound; TCE, trichloroethene; cisDCE, cis-1,2-dichloroethene; VC, vinyl chloride) 

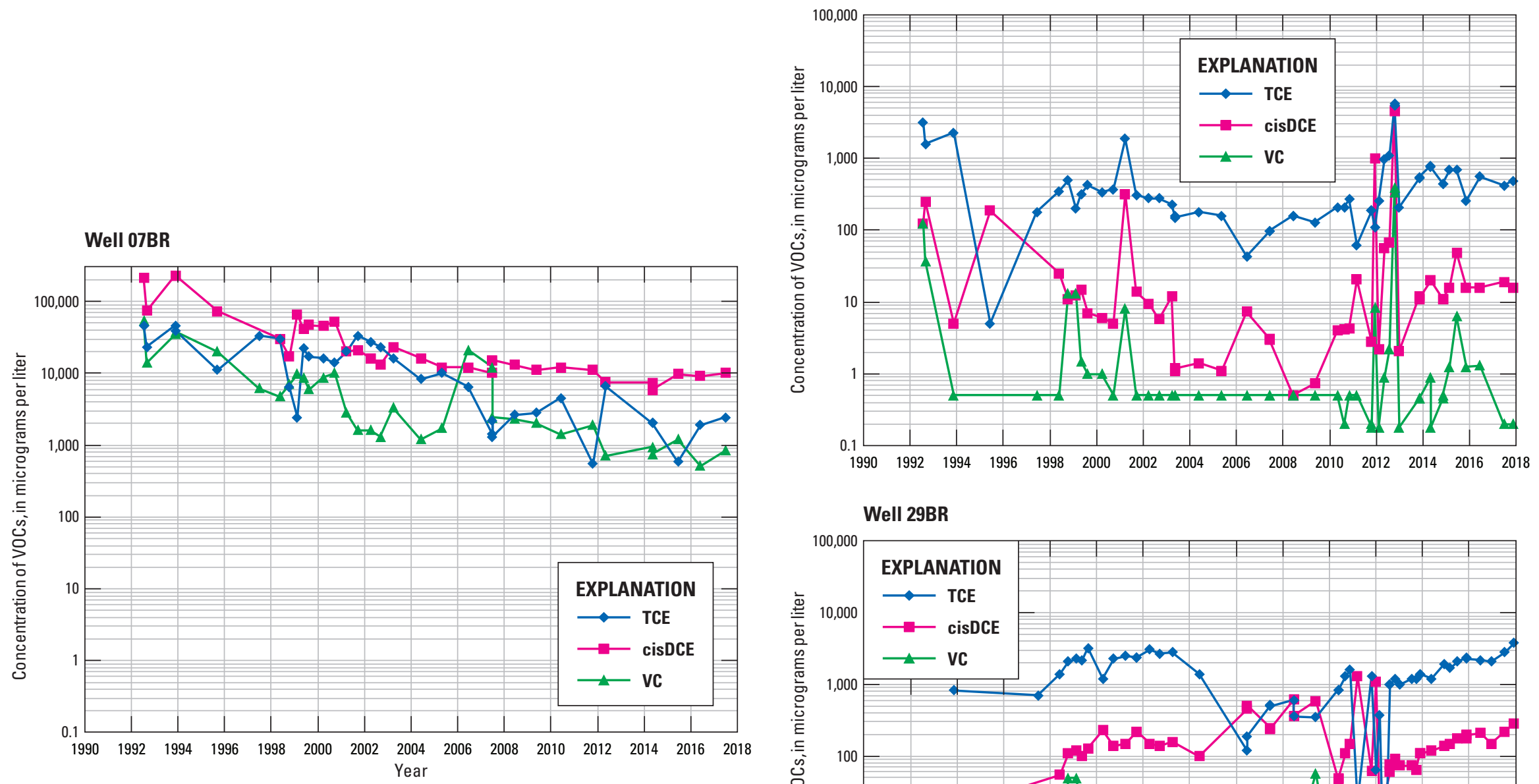

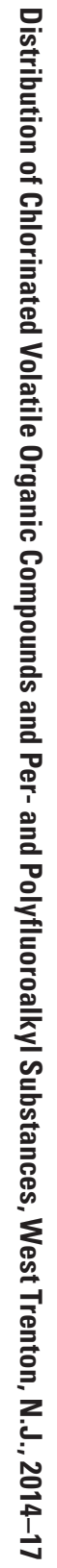

Figure 49. Volatile organic compound concentrations over time in wells along section G-G', former Naval Air Warfare Center, West Trenton, New Jersey, 1992-2017. (VOC,

volatile organic compound; TCE, trichloroethene; cisDCE, cis-1,2-dichloroethene; VC, vinyl chloride)—Continued

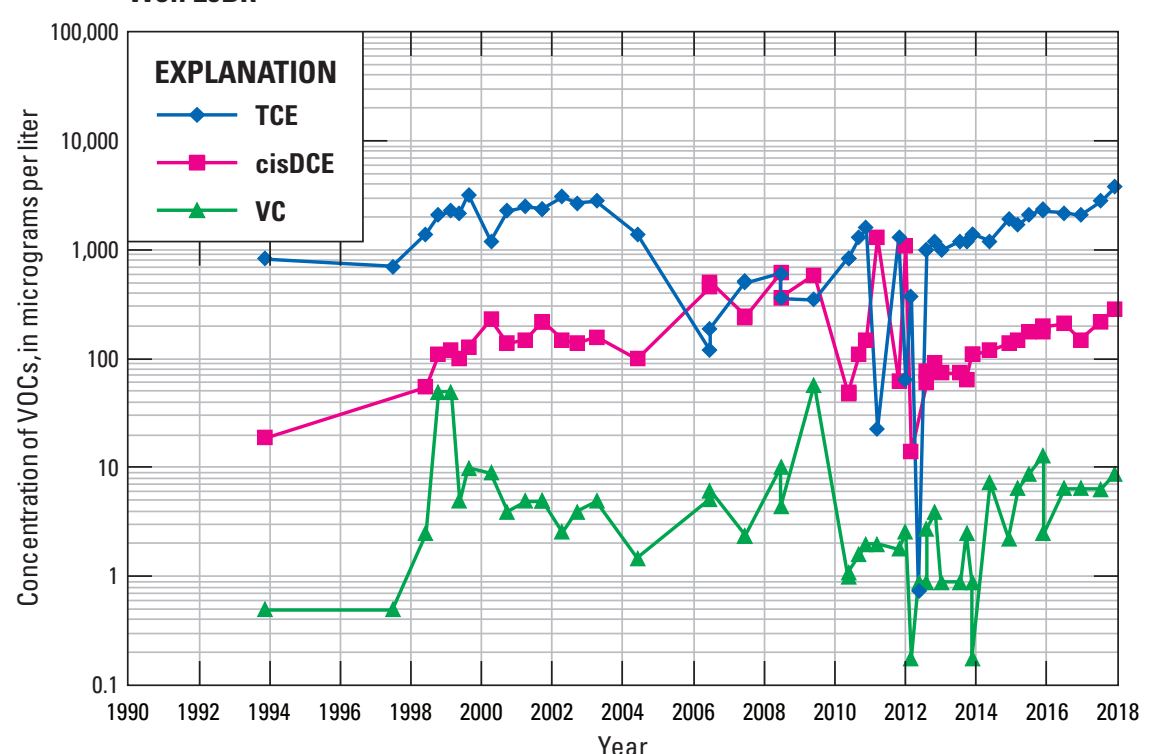




\section{Well 09BR}

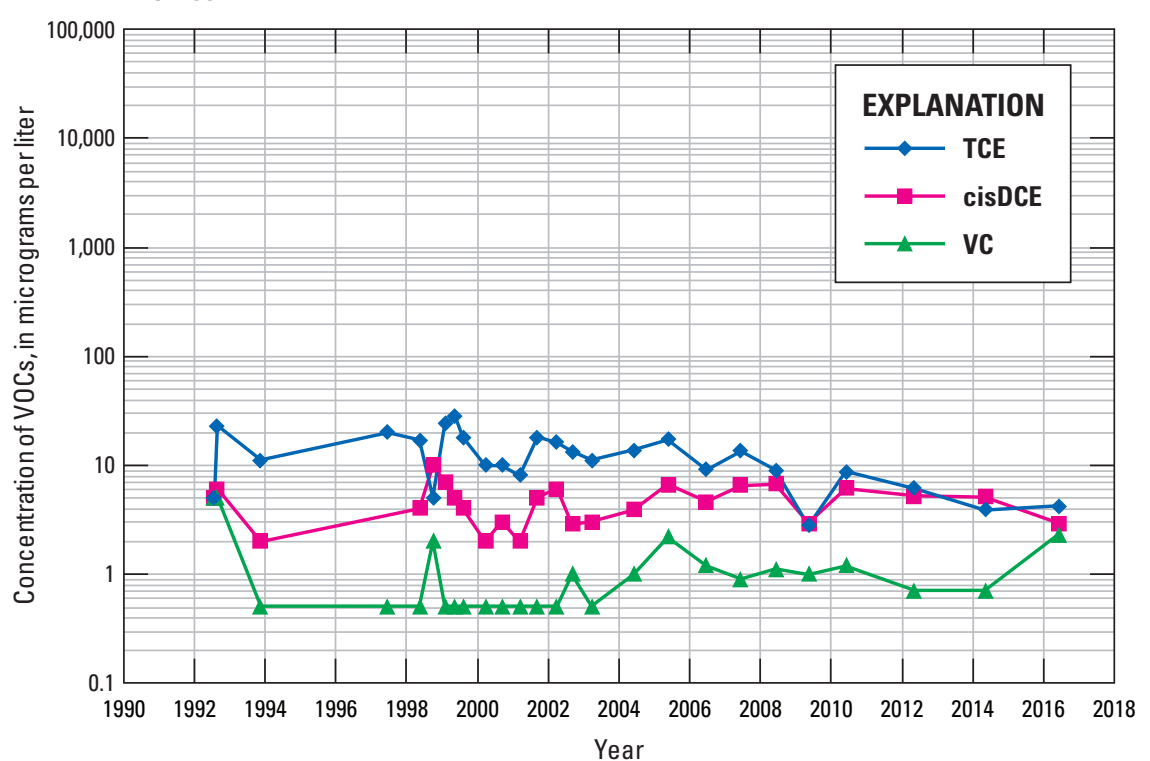

\section{Well 55BR}

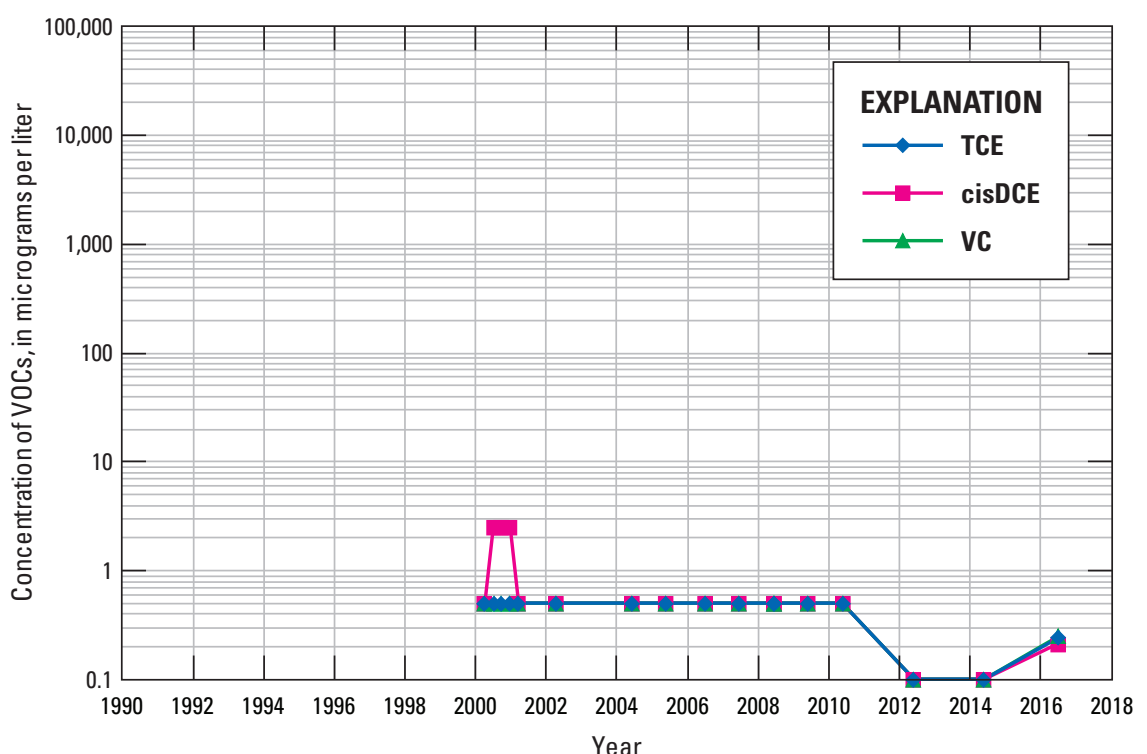

Figure 49. Volatile organic compound concentrations over time in wells along section G-G', former Naval Air Warfare Center, West Trenton, New Jersey, 1992-2017. (VOC, volatile organic compound; TCE, trichloroethene; cisDCE, cis-1,2-dichloroethene; VC, vinyl chloride)—Continued 
Well 05BR

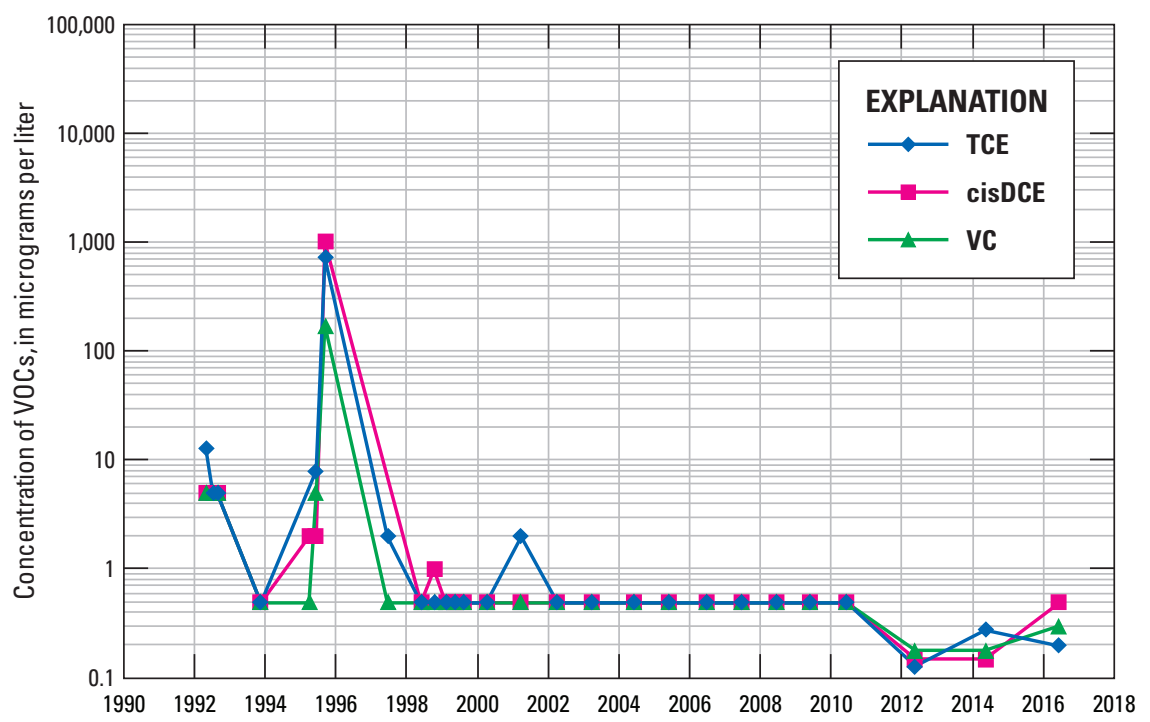

Well 91BR

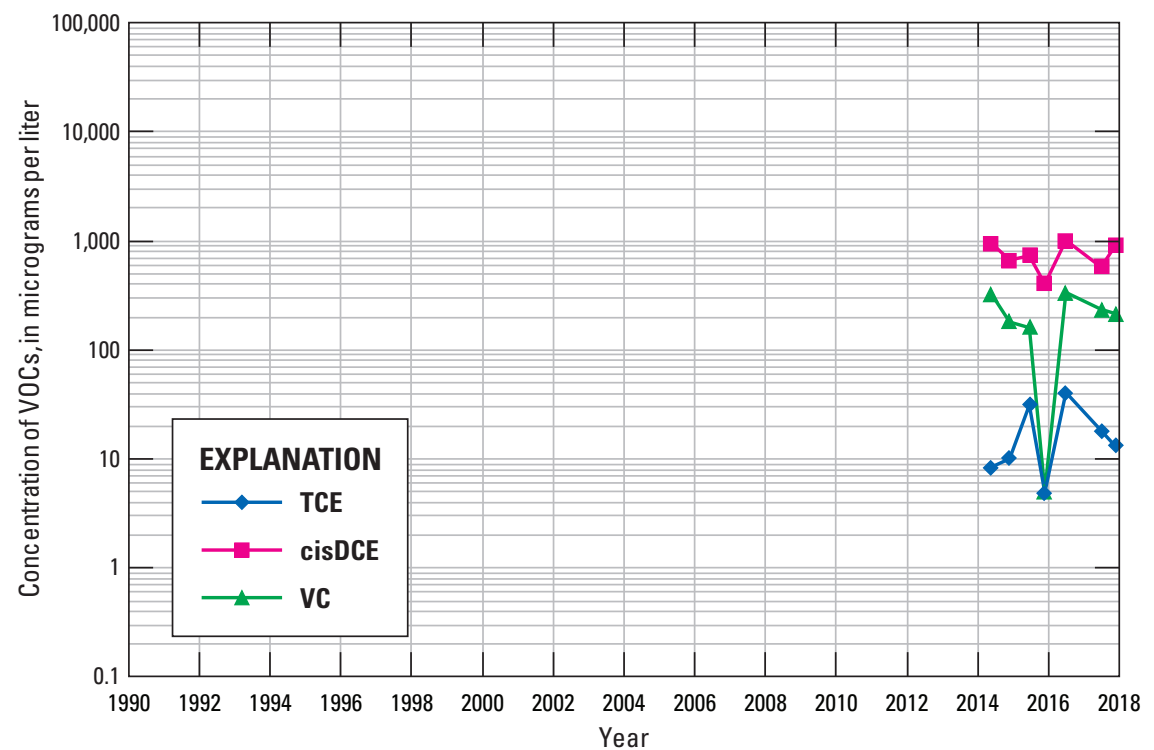

Well 20BR

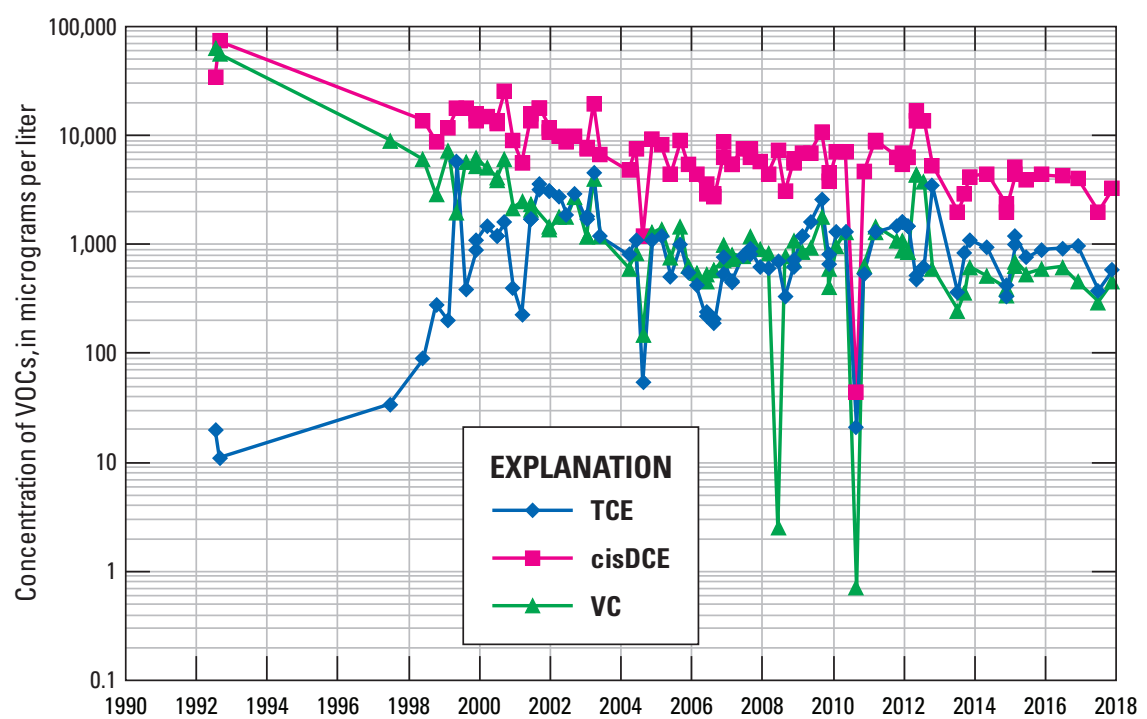

Well BRP02

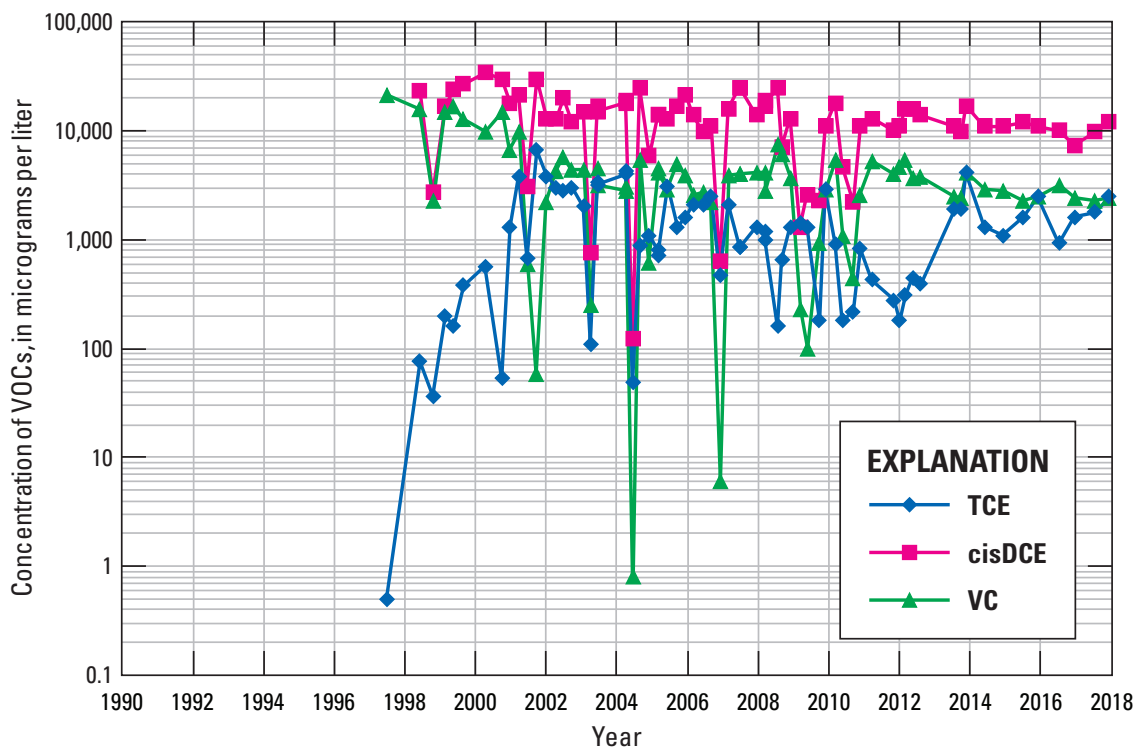

Figure 50. Volatile organic compound concentrations over time in wells along section H-H', former Naval Air Warfare Center, West Trenton, New Jersey, 1992-2017. (VOC, volatile organic compound; TCE, trichloroethene; cisDCE, cis-1,2-dichloroethene; VC, vinyl chloride) 


\section{West Ditch Well}

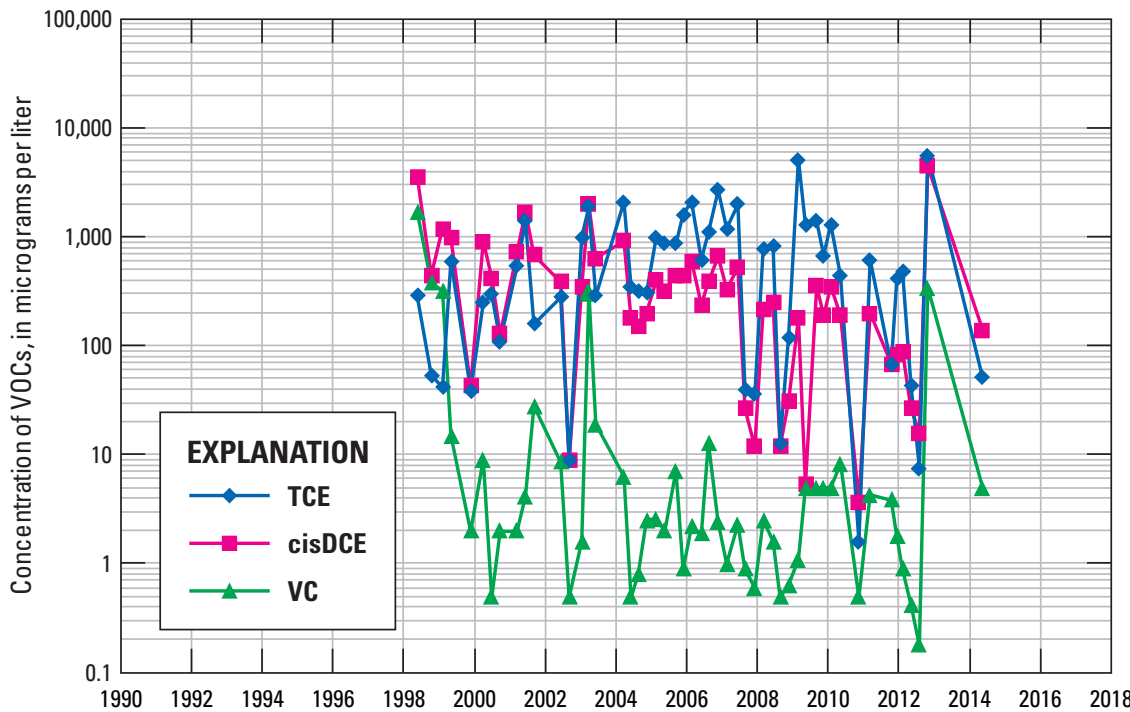

\section{Well 40S}
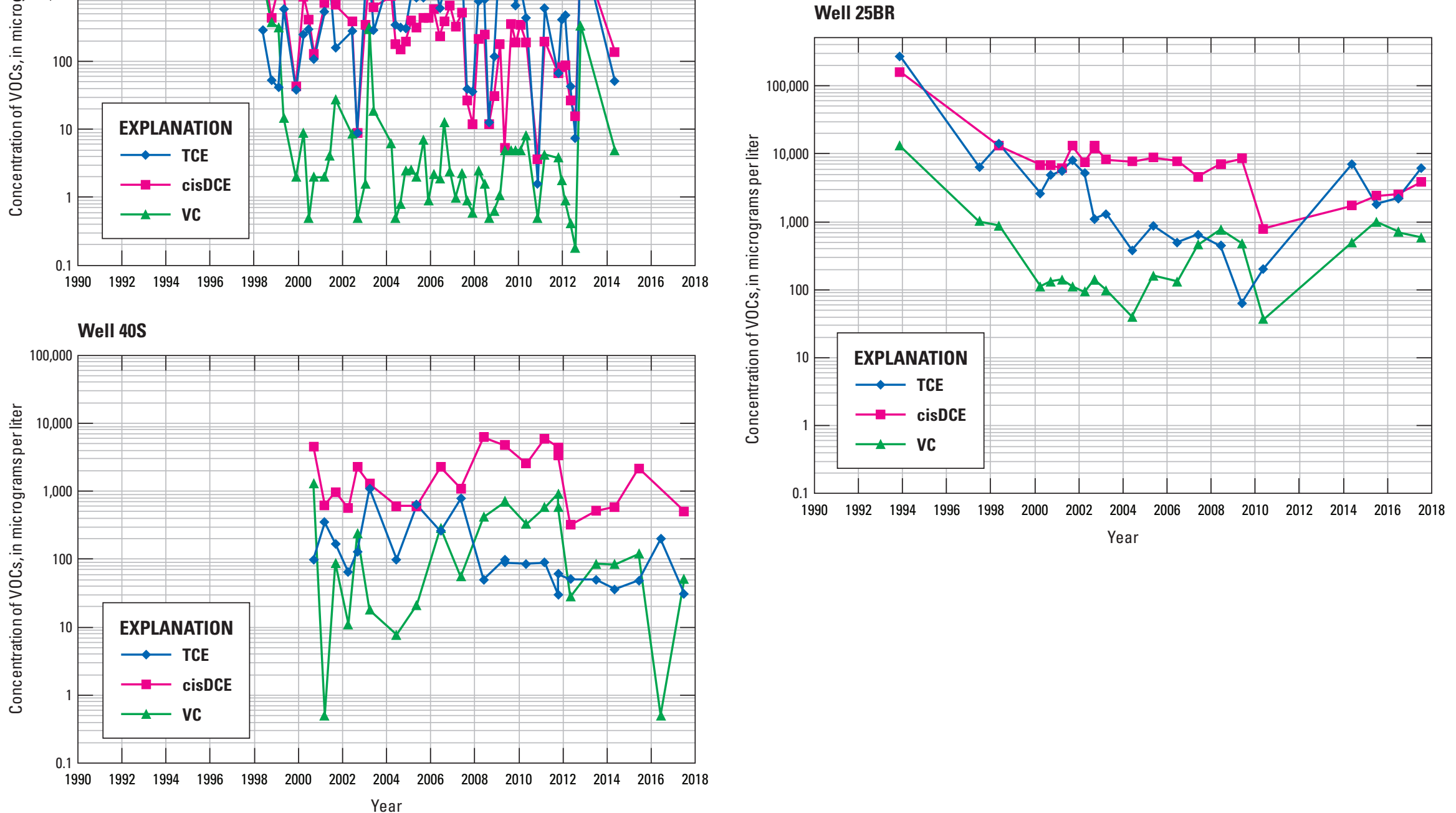

Figure 50. Volatile organic compound concentrations over time in wells along section H-H', former Naval Air Warfare Center, West Trenton, New Jersey, 1992-2017. (VOC, volatile organic compound; TCE, trichloroethene; cisDCE, cis-1,2-dichloroethene; VC, vinyl chloride)—Continued 
Well 15BR

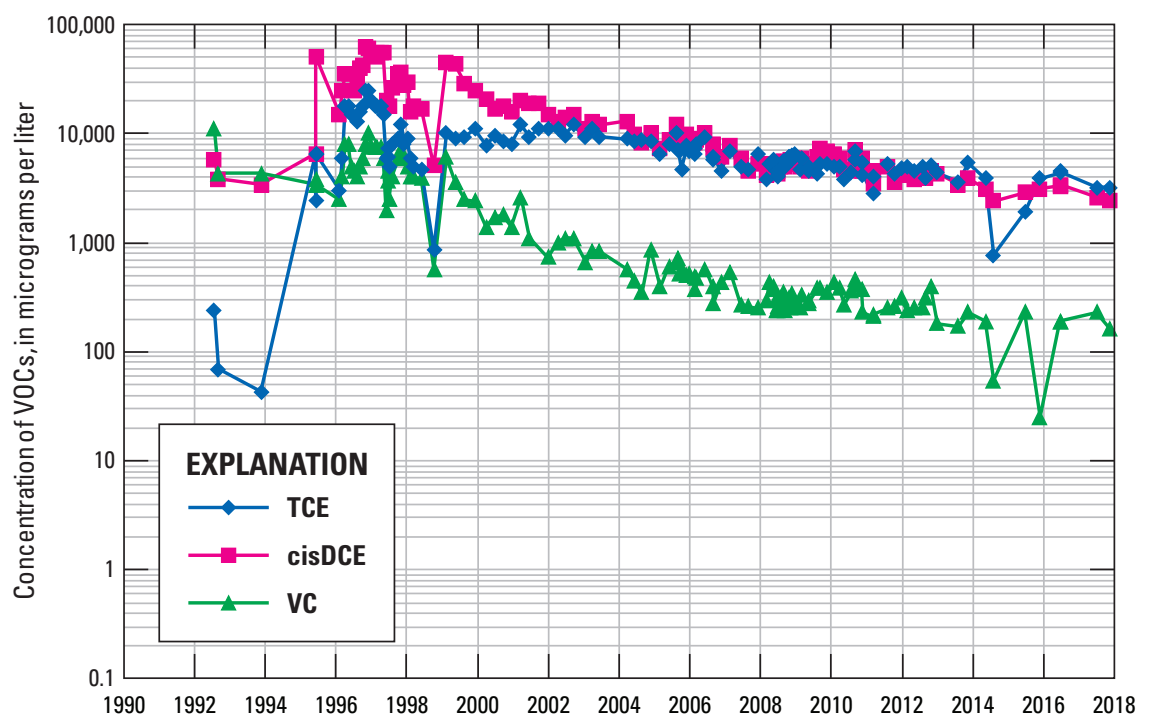

Well 80BR-A

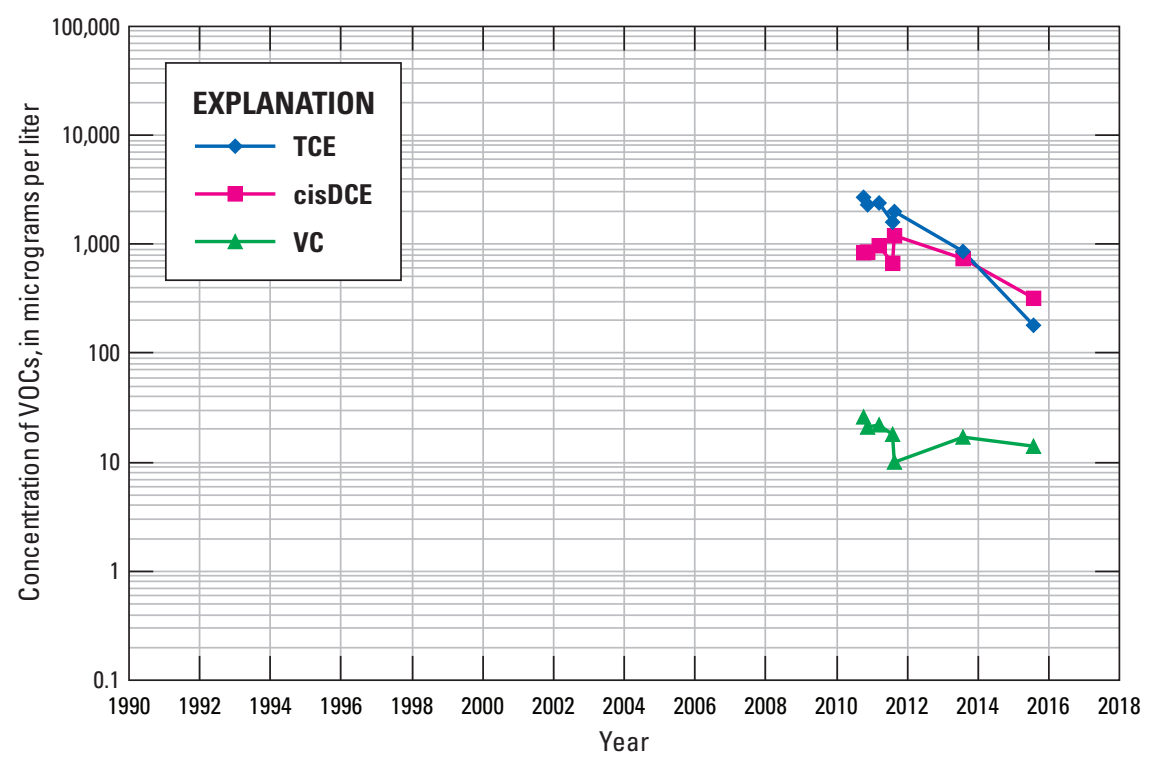

Well BRP03

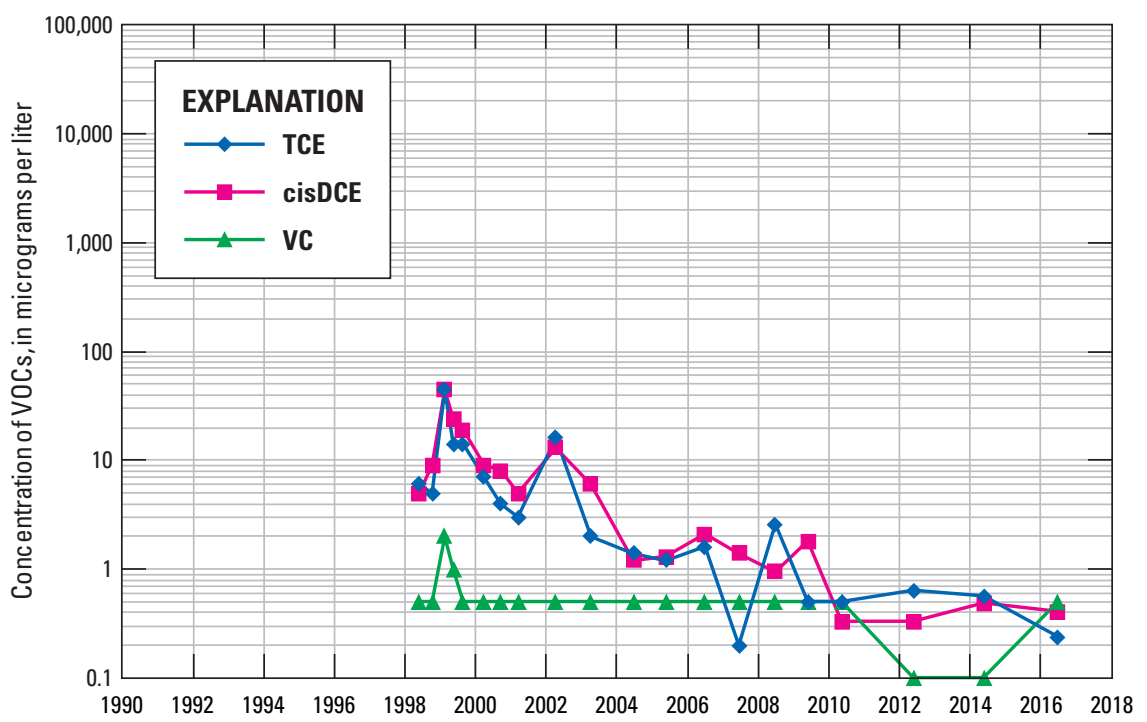

Well 70BR-10

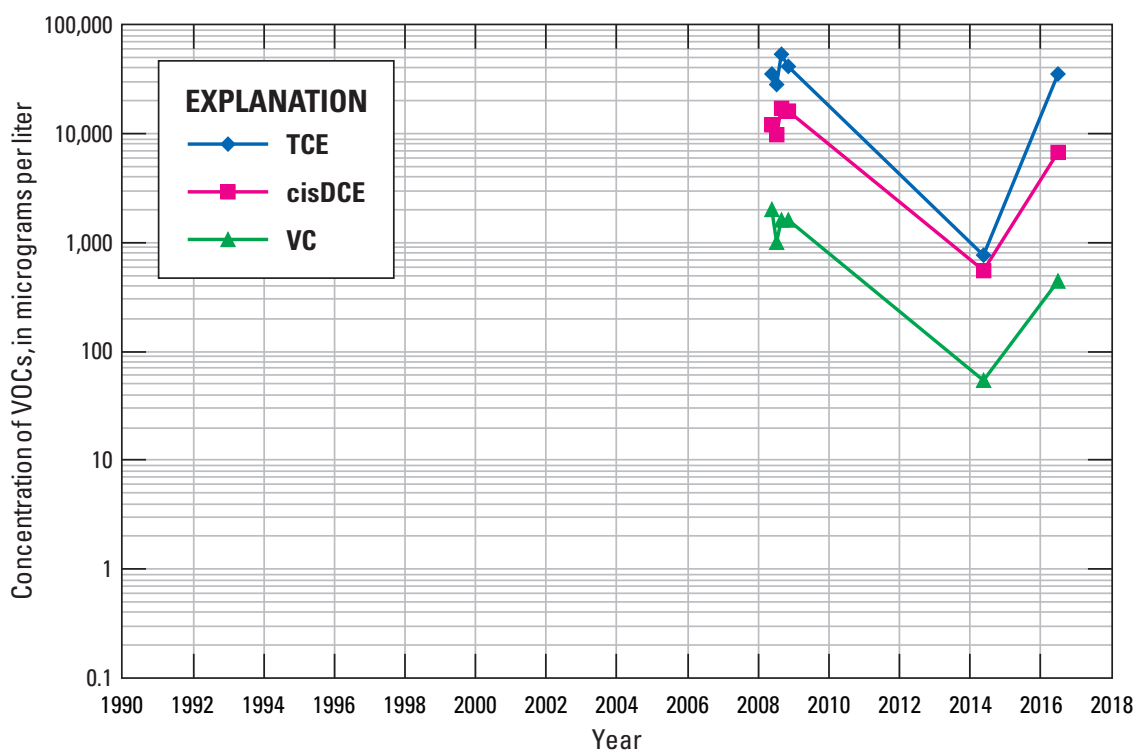

Figure 50. Volatile organic compound concentrations over time in wells along section H-H', former Naval Air Warfare Center, West Trenton, New Jersey, 1992-2017. (VOC, volatile organic compound; TCE, trichloroethene; cisDCE, cis-1,2-dichloroethene; VC, vinyl chloride)—Continued 


\section{Well 70BR-72}

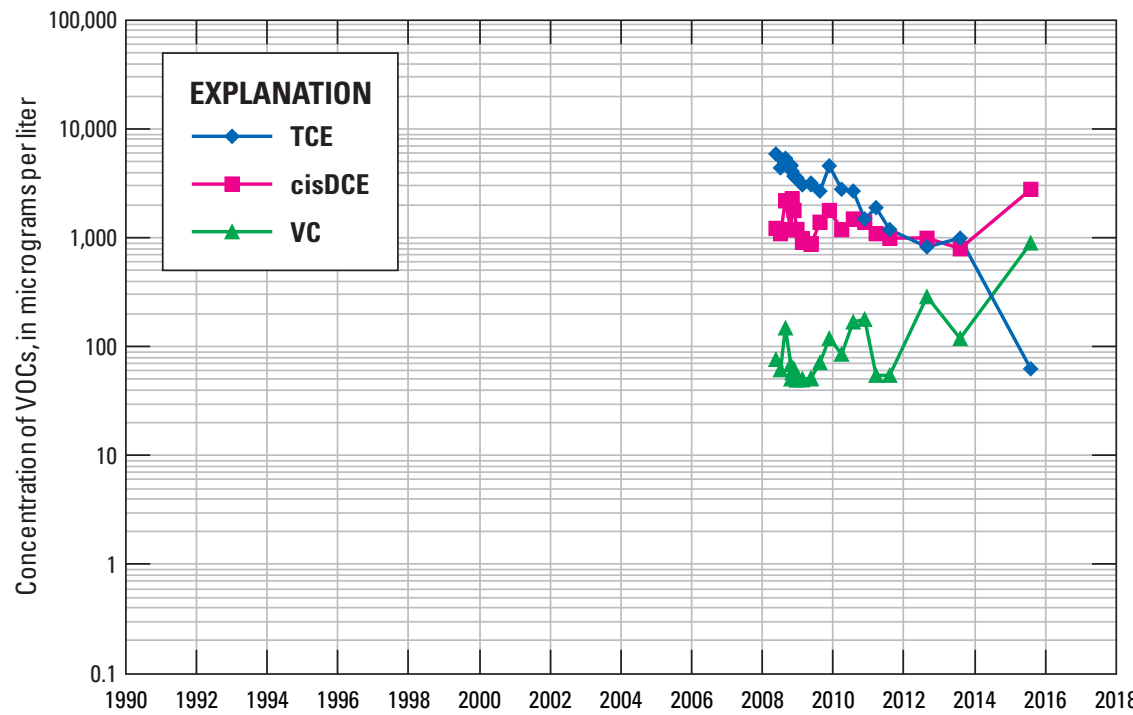

\section{Well 71BR-A}
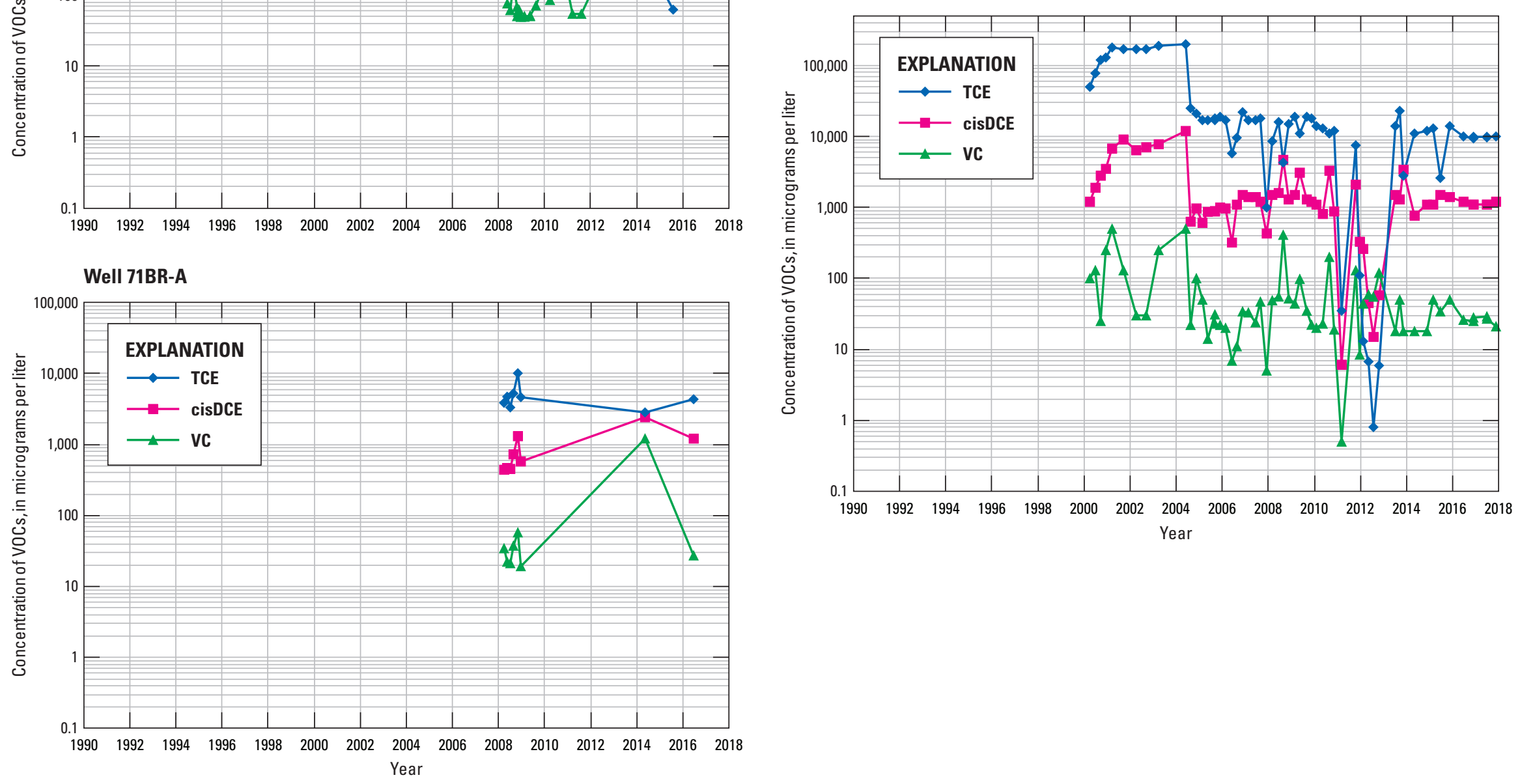

Figure 50. Volatile organic compound concentrations over time in wells along section H-H', former Naval Air Warfare Center, West Trenton, New Jersey, 1992-2017. (VOC, volatile organic compound; TCE, trichloroethene; cisDCE, cis-1,2-dichloroethene; VC, vinyl chloride)—Continued 


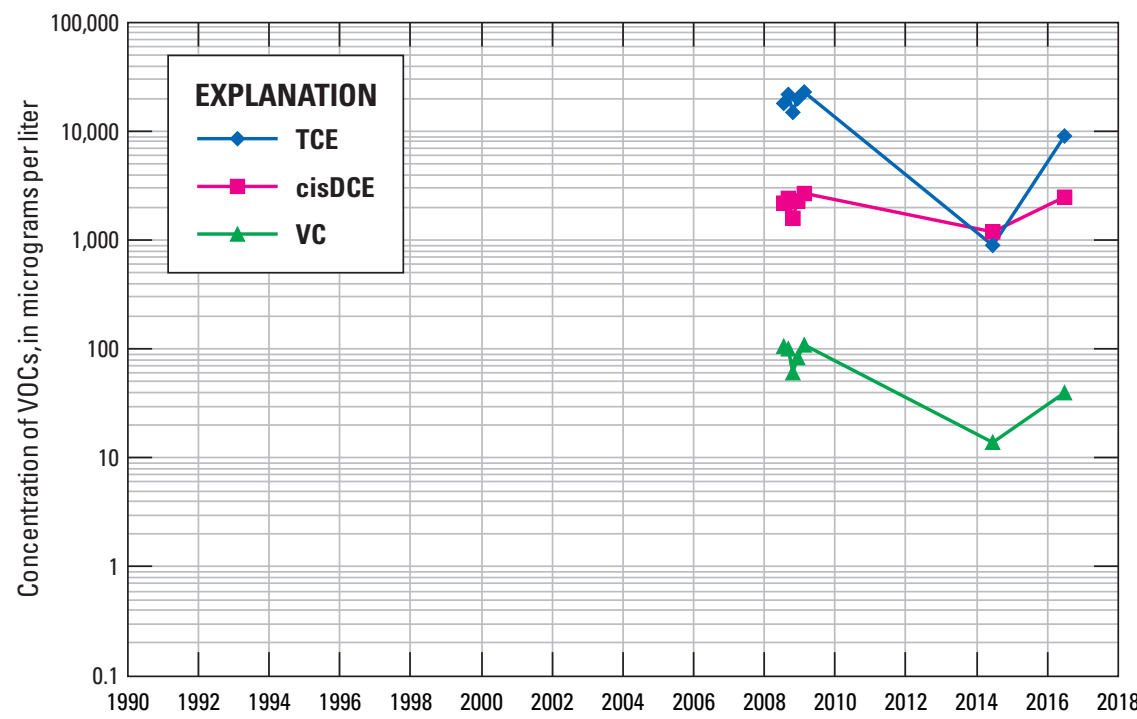

\section{Well 61BR}
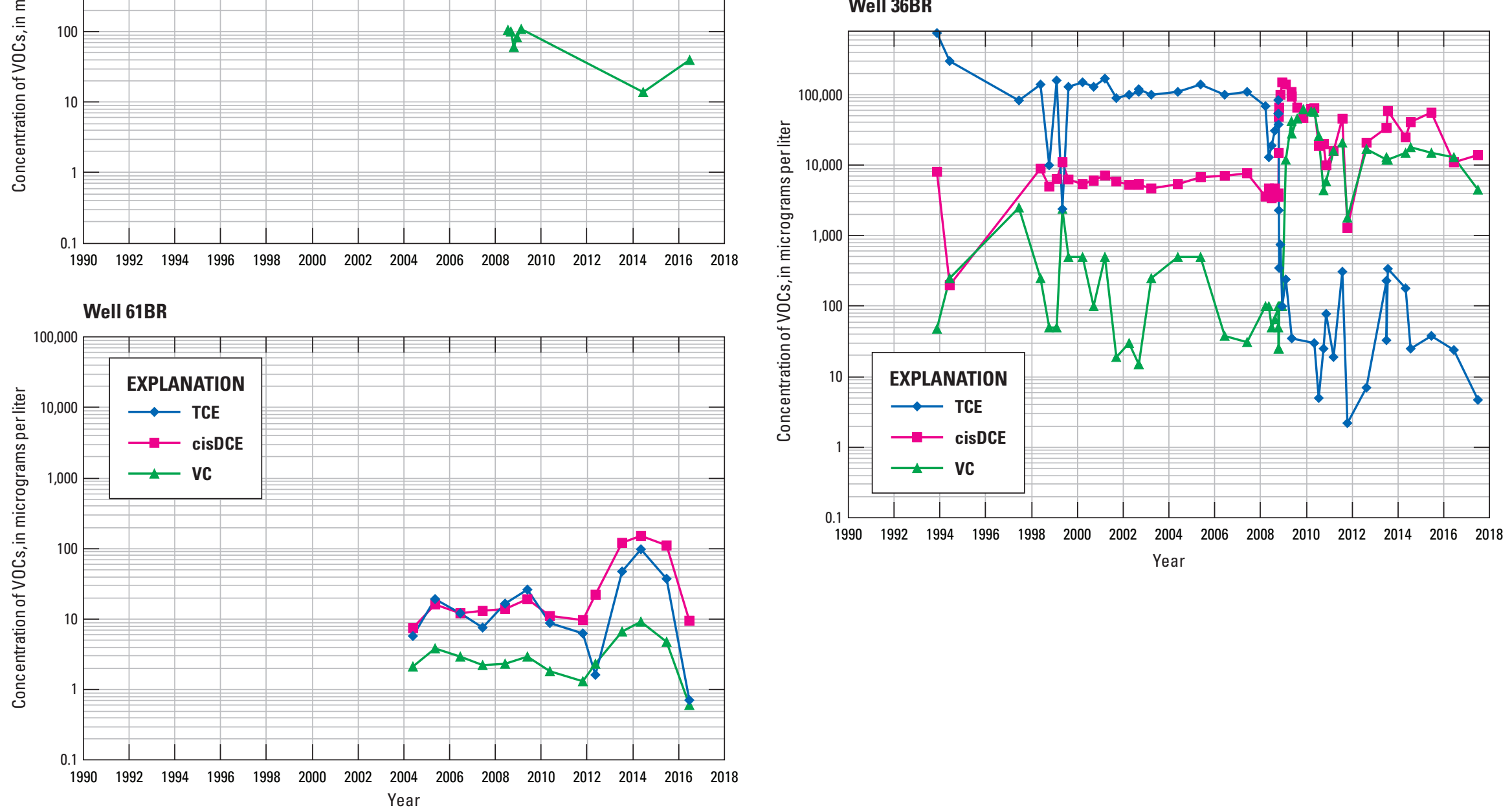

Figure 50. Volatile organic compound concentrations over time in wells along section H-H', former Naval Air Warfare Center, West Trenton, New Jersey, 1992-2017. (VOC, volatile organic compound; TCE, trichloroethene; cisDCE, cis-1,2-dichloroethene; VC, vinyl chloride)—Continued 


\section{Well 45BR}

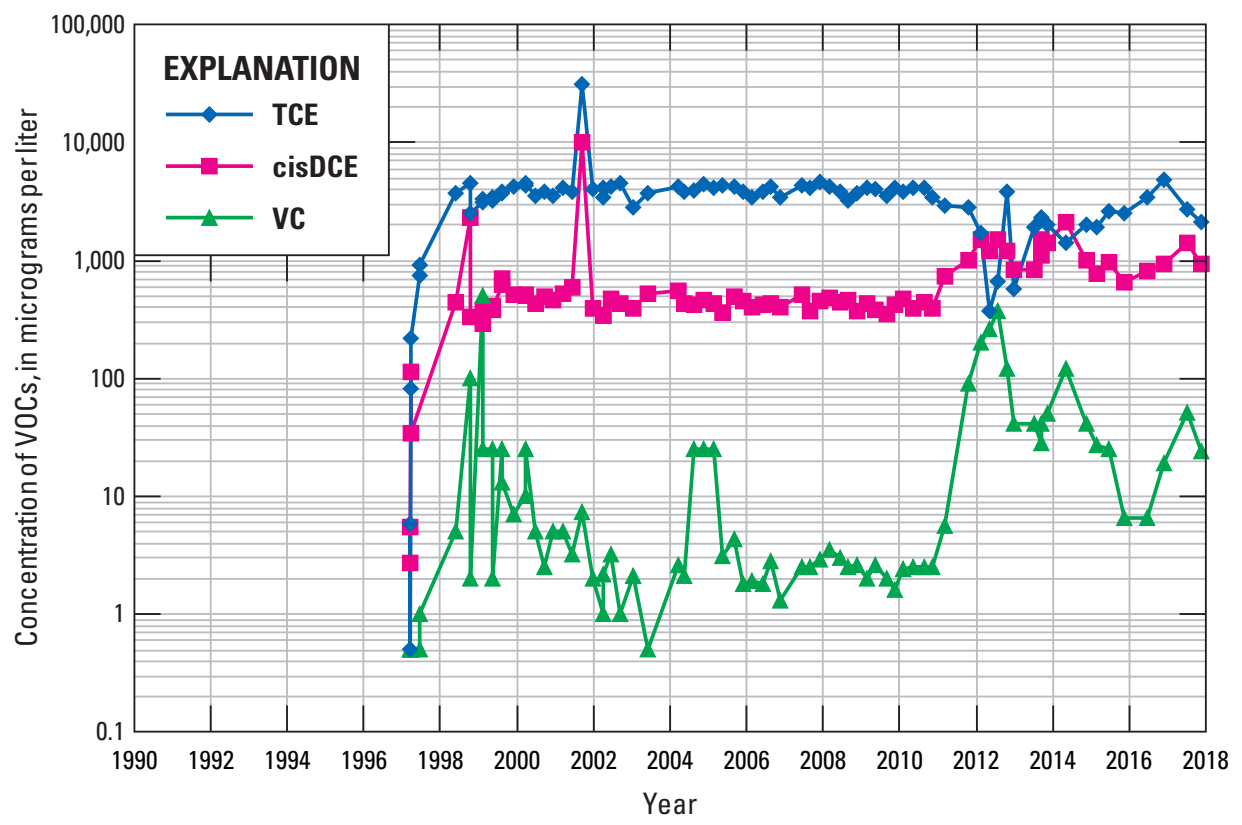

Figure 50. Volatile organic compound concentrations over time in wells along section H-H', former Naval Air Warfare Center, West Trenton, New Jersey, 1992-2017. (VOC, volatile organic compound; TCE, trichloroethene; cisDCE, cis-1,2-dichloroethene; VC, vinyl chloride)Continued 
Well 34BR

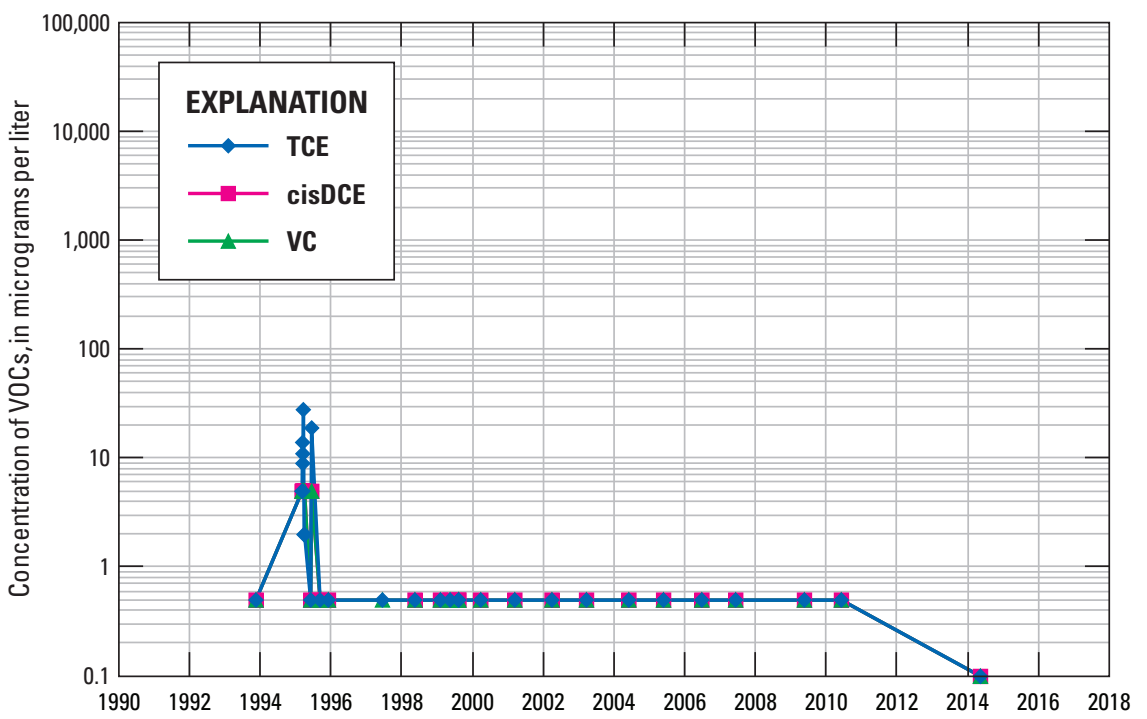

\section{Well 60BR}

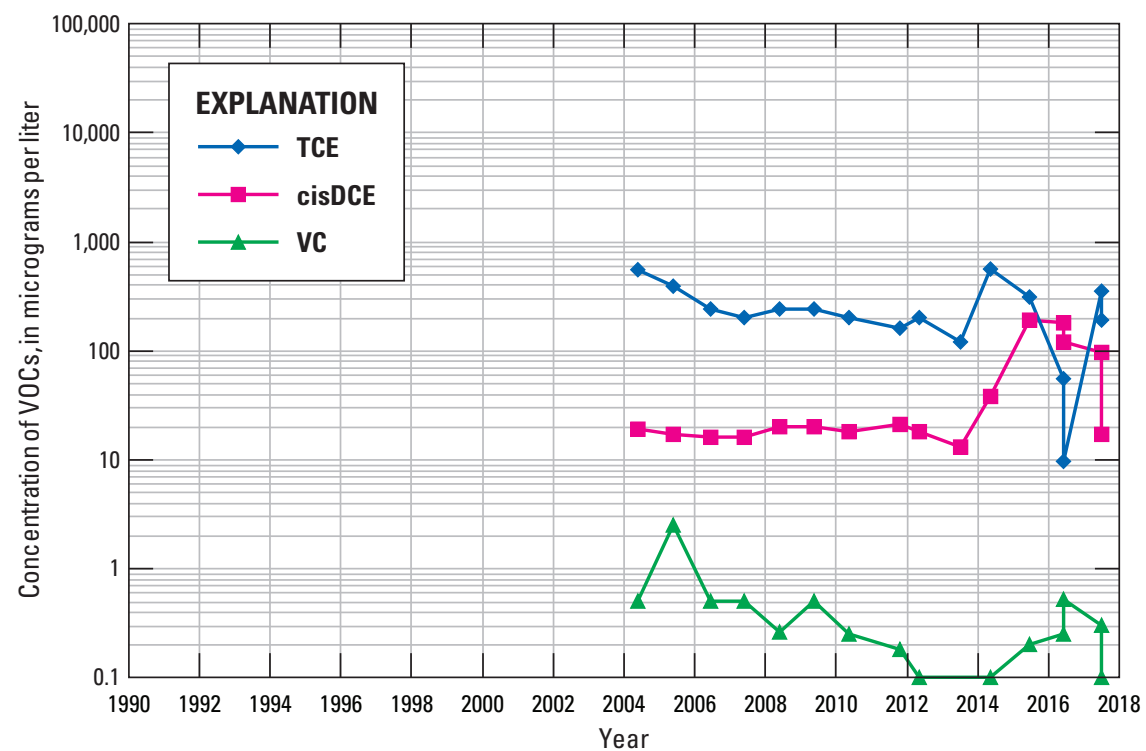

Well 64BR

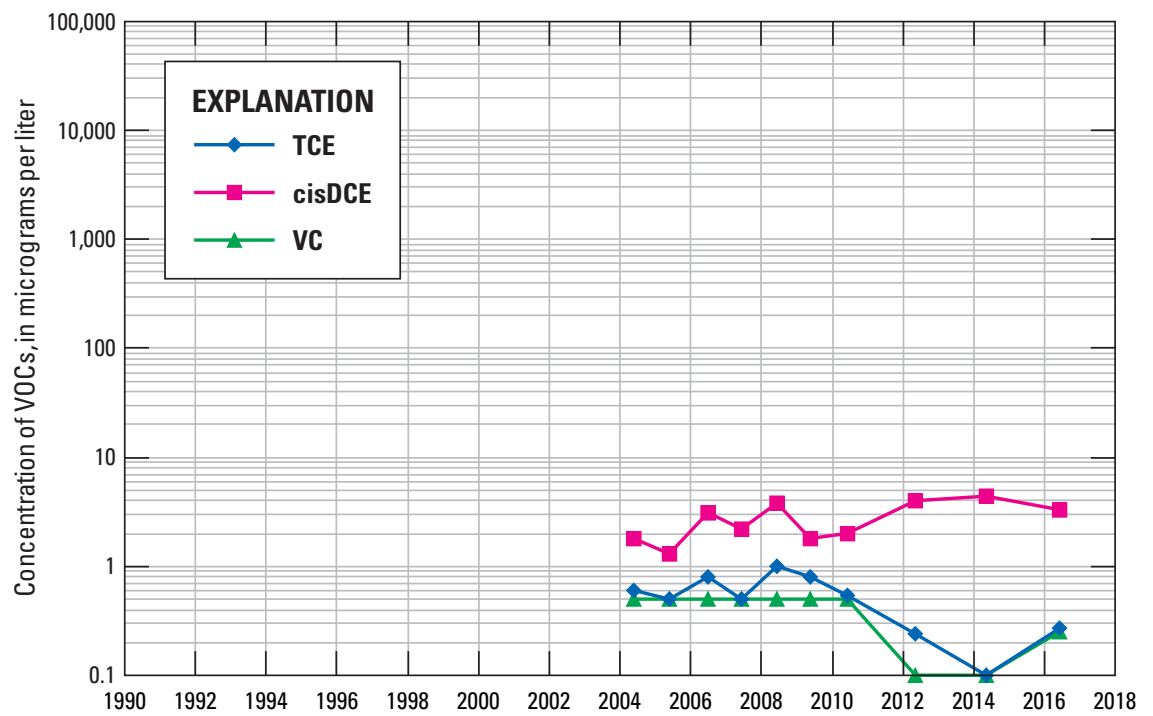

\section{Well 40BR}

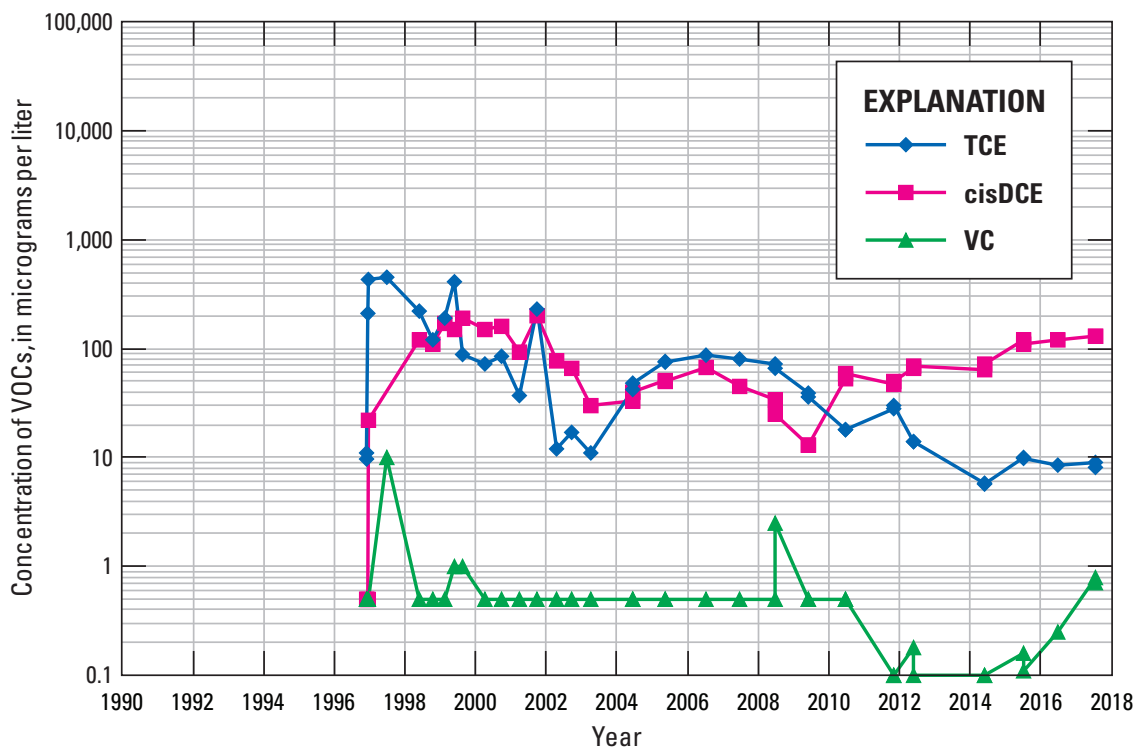

$\underset{N}{\infty}$

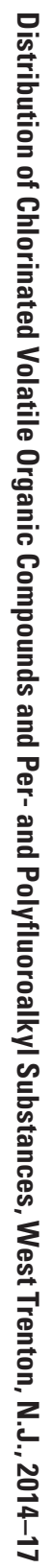

Figure 51. Volatile organic compound concentrations over time in wells along section I-I', former Naval Air Warfare Center, West Trenton, New Jersey, 1993-2017. (V0C, volatile organic compound; TCE, trichloroethene; cisDCE, cis-1,2-dichloroethene; VC, vinyl chloride) 


\section{Well 57BR}

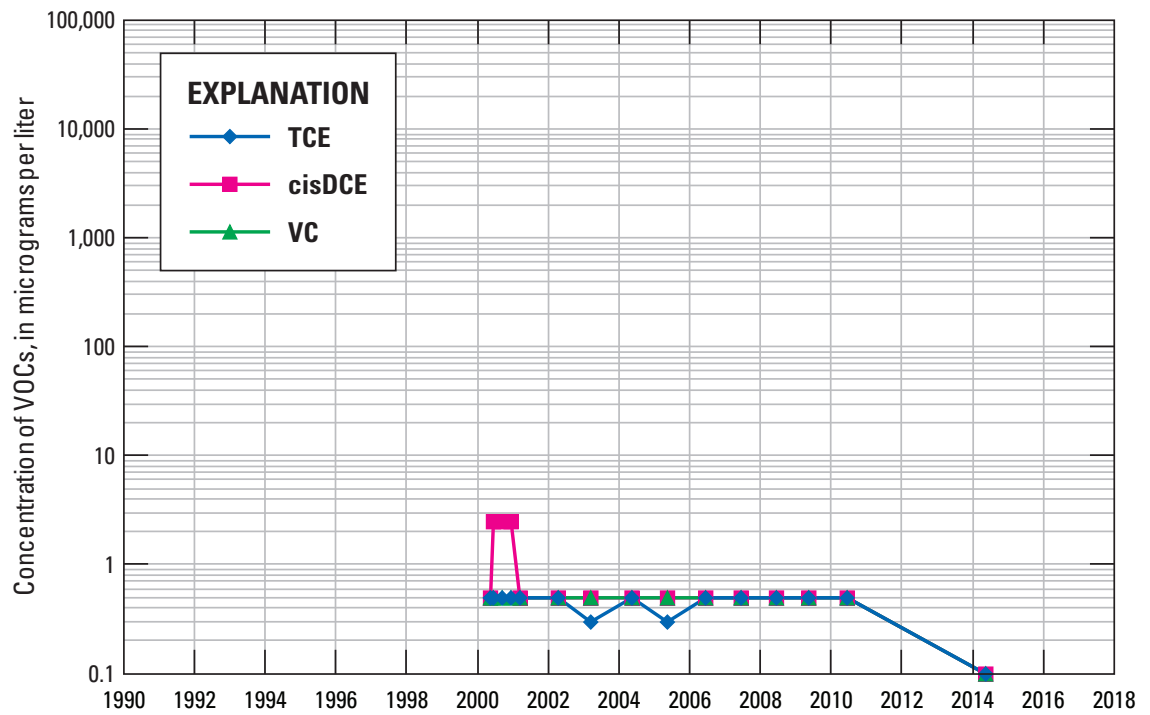

Well 32S

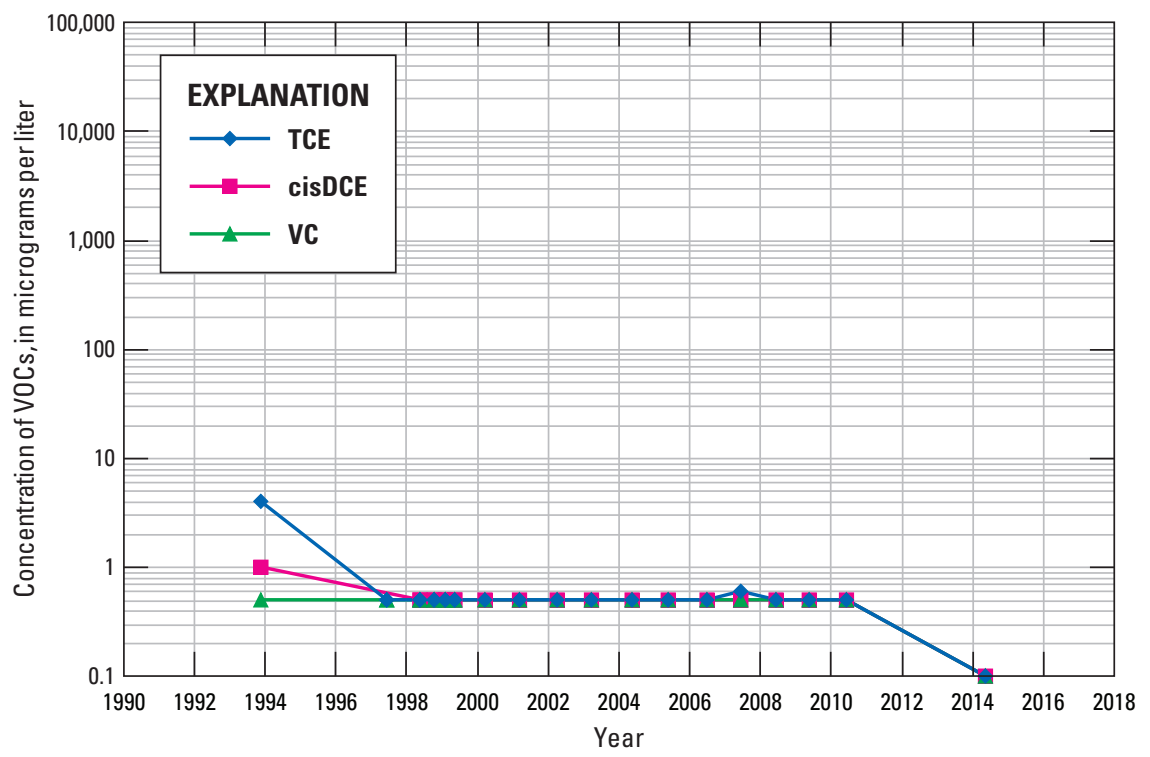

Well 33BR

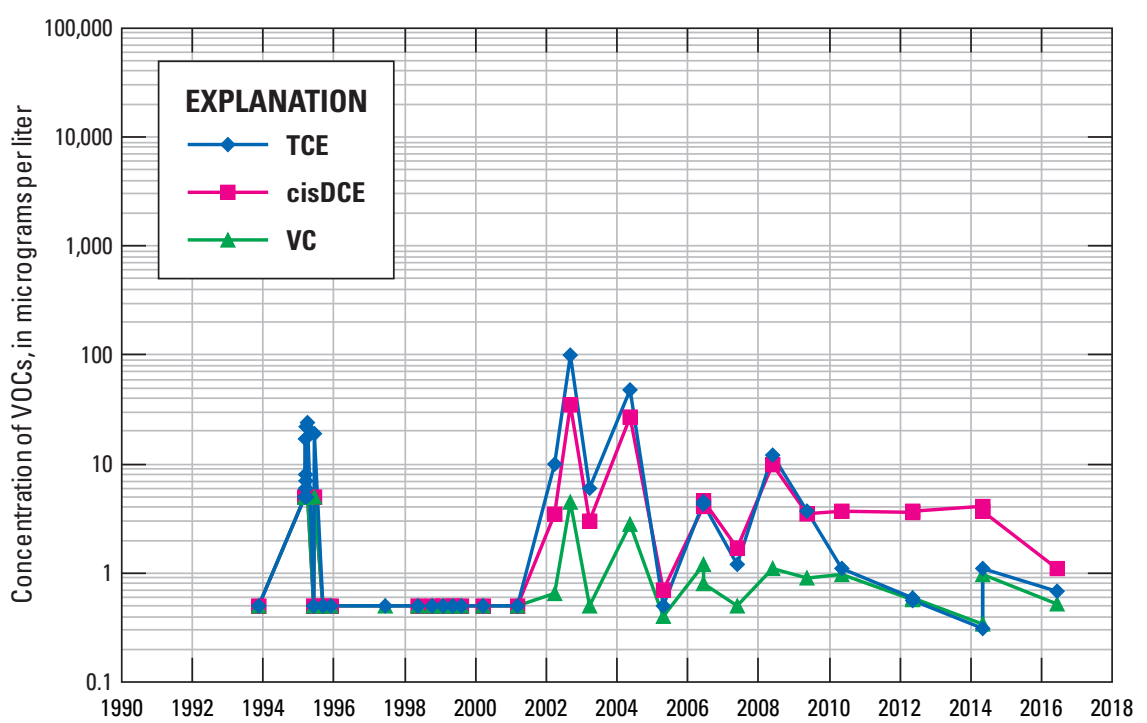

Well 66BR

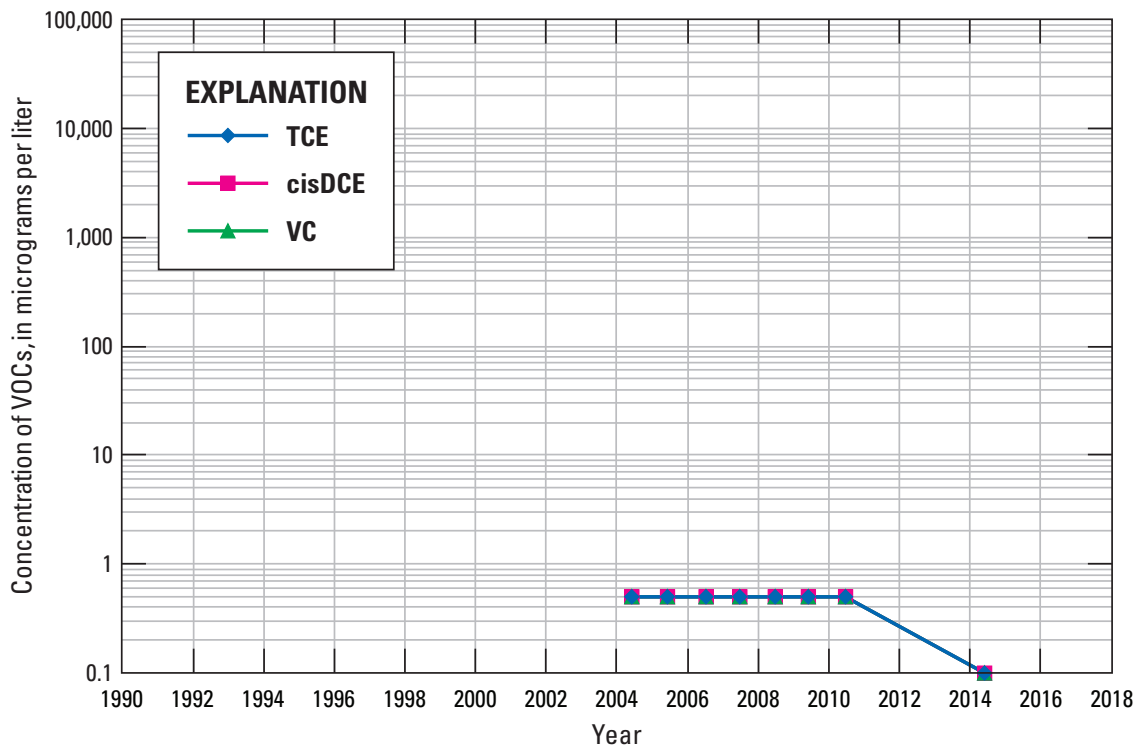

Figure 51. Volatile organic compound concentrations over time in wells along section I-I', former Naval Air Warfare Center, West Trenton, New Jersey, 1993-2017. (V0C, volatile organic compound; TCE, trichloroethene; cisDCE, cis-1,2-dichloroethene; VC, vinyl chloride)—Continued 
Well 62BR

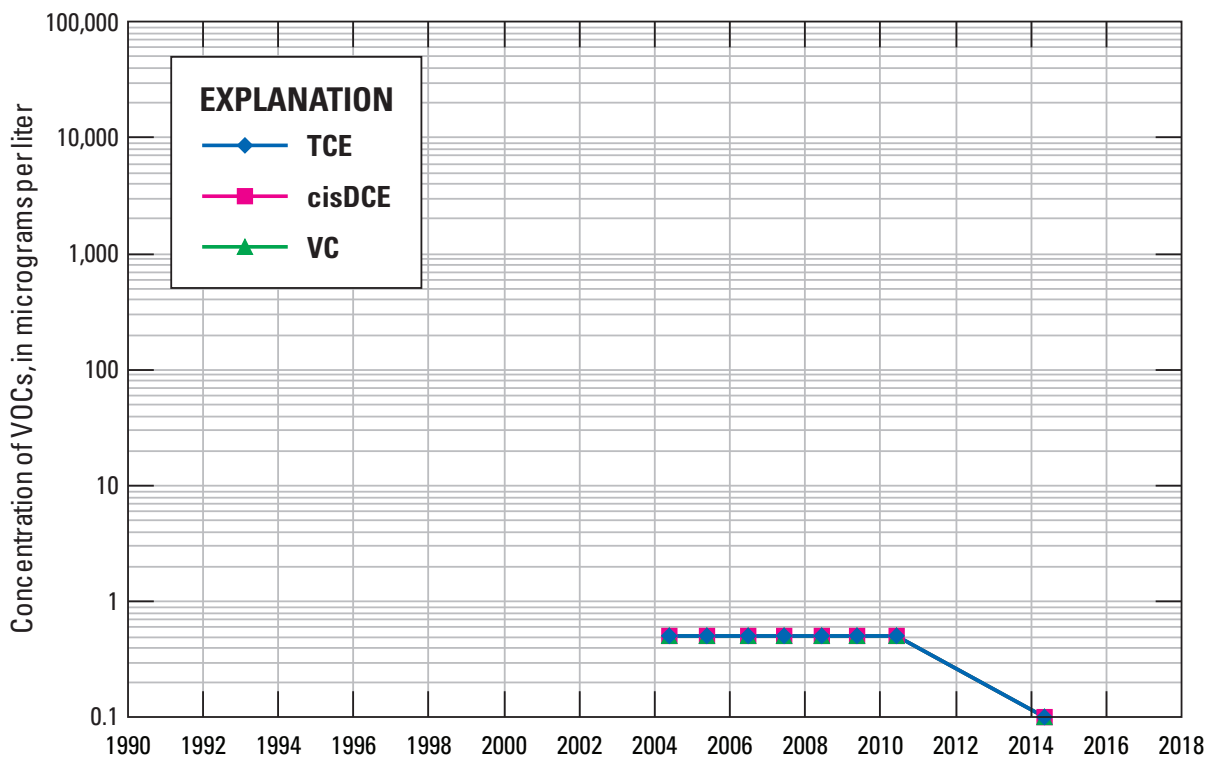

Year

Figure 51. Volatile organic compound concentrations over time in wells along section I-I', former Naval Air Warfare Center, West Trenton, New Jersey, 1993-2017. (VOC, volatile organic compound; TCE, trichloroethene; cisDCE, cis-1,2-dichloroethene; VC, vinyl chloride)-Continued 


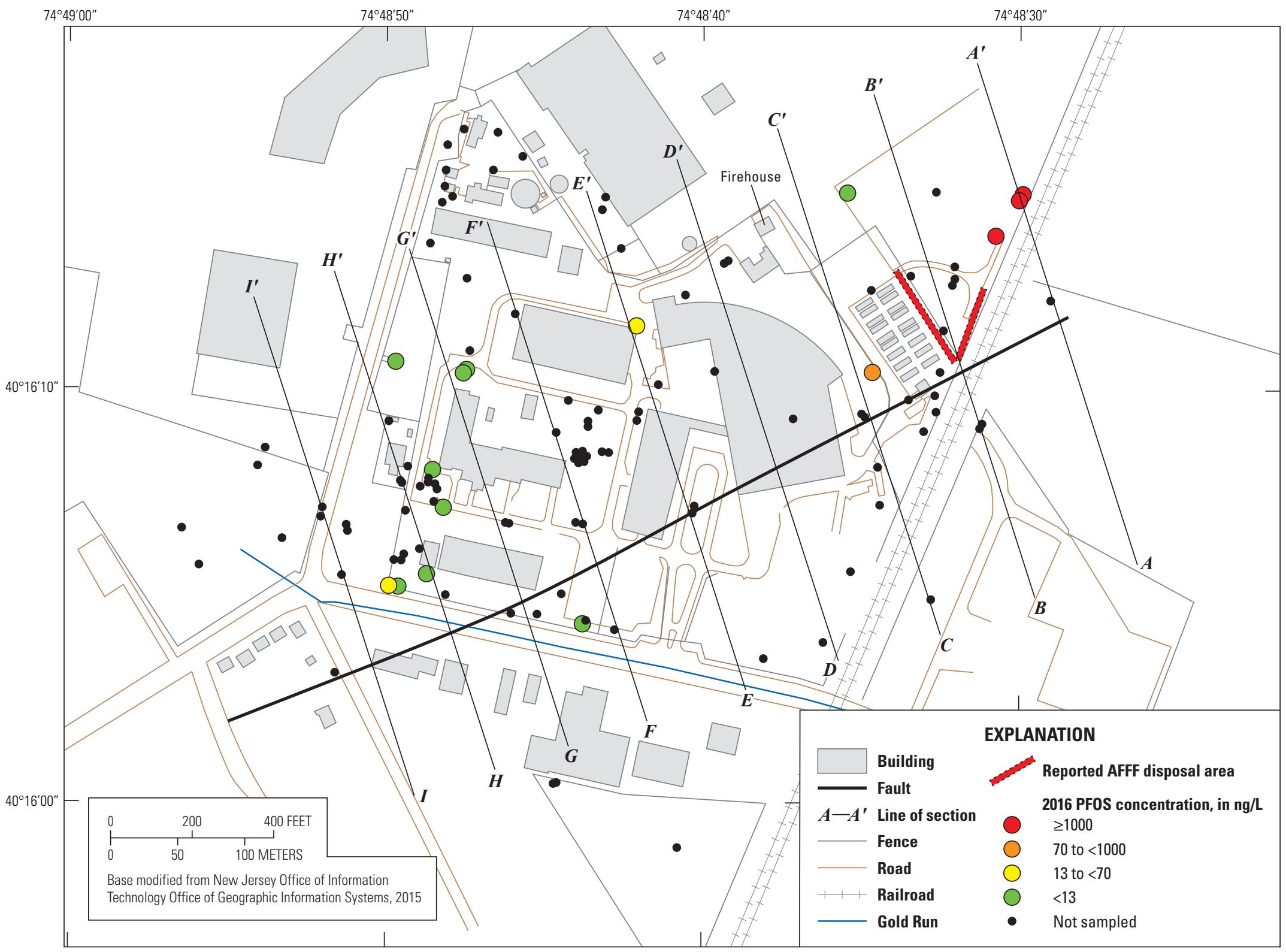

Figure 52. Areal extent of perfluorooctane sulfonate concentrations in wells at the former Naval Air Warfare Center, West Trenton, New Jersey, 2016. (PFOS, perfluorooctane sulfonate; AFFF, aqueous film-forming foam; ng/L, nanograms per liter) 


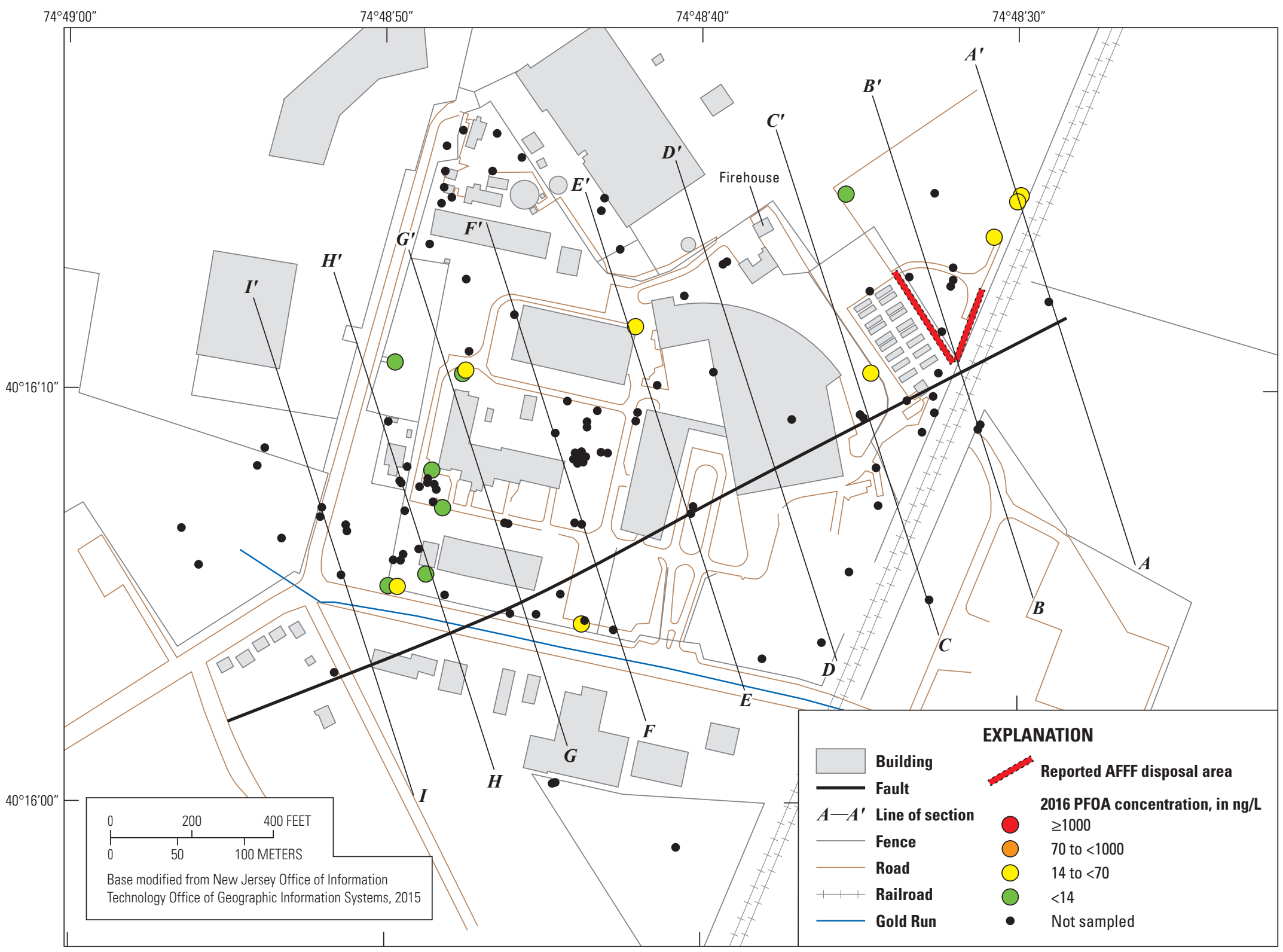

Figure 53. Areal extent of perfluorooctanoic acid concentrations in wells at the former Naval Air Warfare Center, West Trenton, New Jersey, 2016. (PF0A, perfluorooctanoic acid; AFFF, aqueous film-forming foam; ng/L, nanograms per liter) 


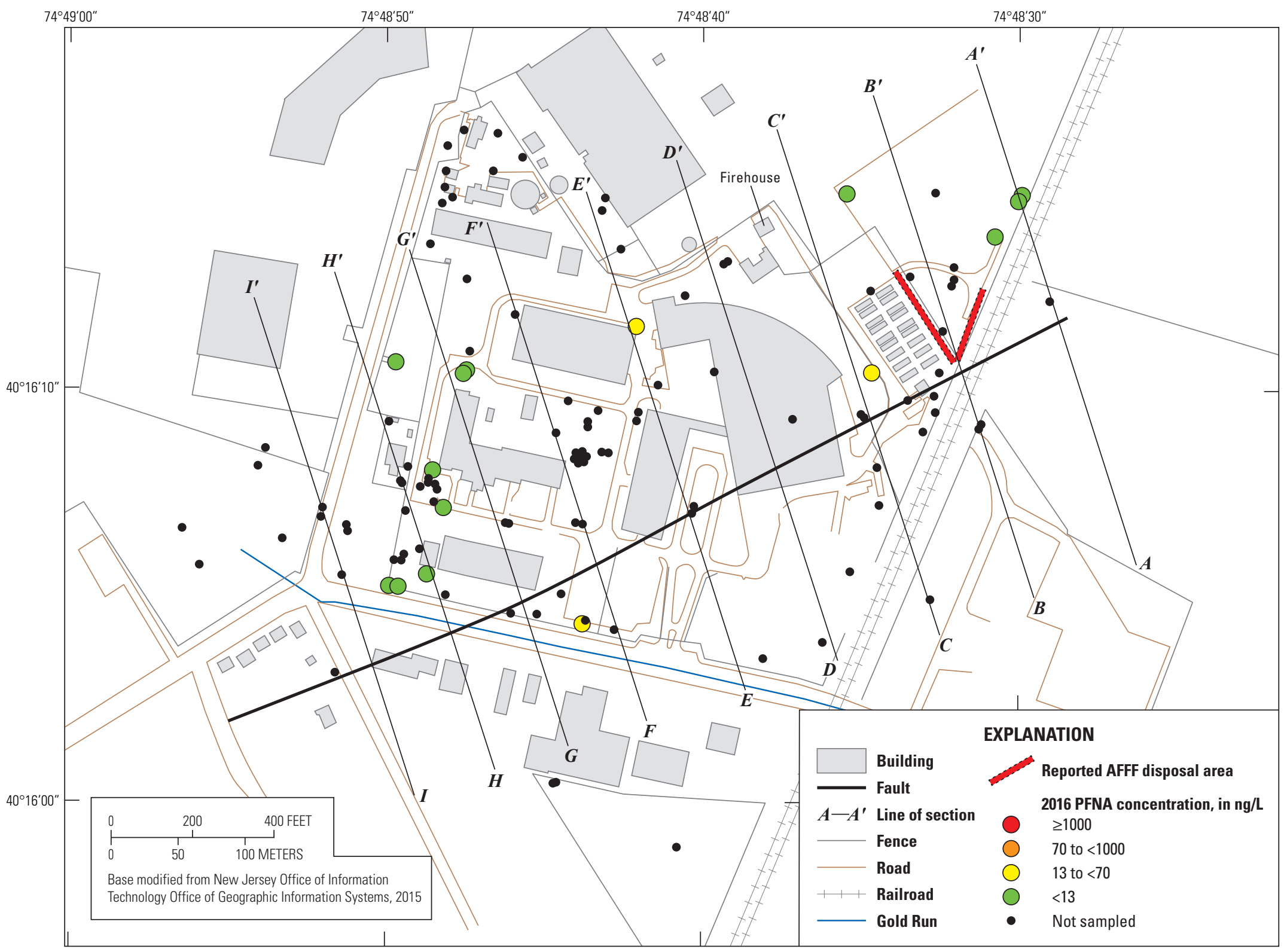

Figure 54. Areal extent of perfluorononanoic acid concentrations in wells at the former Naval Air Warfare Center, West Trenton, New Jersey, 2016. (PFNA, perfluorononanoic acid; AFFF, aqueous film-forming foam; ng/L, nanograms per liter) 
water. He recalled that when he did this test, he sprayed the AFFF along the fence line east of the firehouse and north of the tank farm and along the western side of the railroad tracks north of the tank farm (George Drew, former NAWC fire chief, oral communication, 2016). Wells 11BR, 13S, 14S, and 26S are in these areas. The fire chief estimated that this test of the AFFF was done in approximately 1990.

In 2017, based on the former fire chief's information, all 26 wells on the eastern side of the former NAWC property were sampled for PFAS compounds. The results of this sampling are given in Appendix 2 (Tetra Tech, 2018b) and shown in figures 55,56 , and 57 . PFOS concentrations $\geq 13 \mathrm{ng} / \mathrm{L}$, the NJDEP MCL, were detected in 25 of the 26 wells sampled (fig. 55). Altogether, 24 of the 26 wells sampled contained PFOS concentrations that exceeded the EPA HAL of $70 \mathrm{ng} / \mathrm{L}$. Thirteen wells sampled had PFOS concentrations $\geq 1,000$ $\mathrm{ng} / \mathrm{L}$. PFOS concentrations were highest in two shallow wells along the fence north of the tank farm; a sample from well 11S contained 26,000 ng/L, and a sample from well $27 \mathrm{~S}$ contained $27,000 \mathrm{ng} / \mathrm{L}$. These well locations correspond exactly with the area where the fire chief said he had discharged the AFFF. PFOS was present in concentrations $\geq 13 \mathrm{ng} / \mathrm{L}$ in four wells on the eastern side of the railroad tracks, indicating that the contamination had traveled offsite. In addition, PFOS was present in concentrations $\geq 13 \mathrm{ng} / \mathrm{L}$ in 11 wells south of the fault. PFOS contamination may have traveled with shallow groundwater through the overburden or with surface-water runoff through leaking storm sewers to spread from the northern side of the fault to the southern side of the fault.

PFOA concentrations were detected in most of the same wells as PFOS concentrations were detected, though generally at an order of magnitude less (fig. 56). The highest PFOA concentrations were present in wells $11 \mathrm{~S}(2,000 \mathrm{ng} / \mathrm{L})$ and $27 \mathrm{~S}(600 \mathrm{ng} / \mathrm{L})$, which are the wells where the highest PFOS concentrations were present, indicating that PFOA and PFOS originated from a common source, presumably the discharge of the AFFF previously described. PFOA concentrations exceeding the NJDEP MCL of $14 \mathrm{ng} / \mathrm{L}$ were present in 24 of the 26 wells sampled on the eastern side of the NAWC site. PFOA concentrations exceeding the EPA HAL of $70 \mathrm{ng} / \mathrm{L}$ were present in 15 of the 26 wells sampled on the eastern side of the NAWC site. PFOA was present in the same four wells as PFOS on the eastern side of the railroad tracks at concentrations $\geq 14 \mathrm{ng} / \mathrm{L}$, indicating that PFOA had moved offsite to the east. PFOA concentrations $\geq 14 \mathrm{ng} / \mathrm{L}$ were present in 11 wells south of the fault. Like PFOS, PFOA may have traveled with the shallow groundwater through the overburden or with surface-water runoff through leaking storm sewers to spread from the northern side of the fault to the southern side of the fault.

PFNA concentrations $\geq 13 \mathrm{ng} / \mathrm{L}$ were detected in 12 of the 26 wells sampled at the former NAWC site in 2017 (fig. 57). All PFNA detections occurred in wells where PFOS and PFOA were also detected. The concentrations of PFNA in most wells were generally lower than the PFOA concentrations by an order of magnitude (Appendix 2). The highest
PFNA concentrations were present in wells $11 \mathrm{~S}(480 \mathrm{ng} / \mathrm{L})$, 12S (190-210 ng/L), and 02BR (110 ng/L). Well 11S also had the highest PFOS and PFOA concentrations. The distribution of elevated concentrations of PFNA was not as areally extensive as that of PFOS and PFOA (fig. 57). No wells to the east of the railroad tracks had PFNA concentrations $\geq 13 \mathrm{ng} / \mathrm{L}$. Only eight wells south of the fault had PFNA concentrations $\geq 13 \mathrm{ng} / \mathrm{L}$.

\section{Vertical Extent of PFAS Contamination in 2017}

PFOS concentrations in 24 of 25 wells along sections A-A' through D-D' (figs. 58 and 59) exceeded the EPA HAL of $70 \mathrm{ng} / \mathrm{L}$. PFOS concentrations in all 25 wells along sections A-A' through D-D' exceeded the NJDEP MCL of $13 \mathrm{ng} / \mathrm{L}$. These high concentrations extended vertically from shallow groundwater to $200 \mathrm{ft}$ below land surface in well 54BR. Most of the higher PFOS concentrations were detected in shallow overburden wells along these sections. The higher shallow PFOS contamination is consistent with AFFF having been applied at the land surface. Deep wells, such as 02BR, 51BR, and 54BR, were most likely contaminated by downward seepage of PFOS-contaminated groundwater along the higher transmissivity bedding partings noted on the cross sections (figs. 58 and 59).

PFOA concentrations in 15 of 25 wells along sections A-A' through D-D' (figs. 60 and 61) exceeded the EPA HAL of $70 \mathrm{ng} / \mathrm{L}$. PFOA concentrations in 24 of 25 wells along sections A-A' through D-D' exceeded the NJDEP MCL of $14 \mathrm{ng} / \mathrm{L}$. These high concentrations extended vertically from the shallow groundwater to $200 \mathrm{ft}$ below land surface in well 54BR. The high PFOA concentrations in most shallow overburden wells along these sections are consistent with AFFF having been applied at the land surface. Like the vertical distribution of PFOS, deeper wells, such as 02BR, 51BR, and 54BR, were likely contaminated by downward seepage of PFOA-contaminated groundwater along the higher transmissivity bedding partings noted on cross sections (figs. 60 and 61).

PFNA concentrations in 12 of 25 wells along sections A-A' through D-D' (figs. 62 and 63) exceeded the NJDEP $\mathrm{MCL}$ of $13 \mathrm{ng} / \mathrm{L}$. These high concentrations extended vertically from shallow groundwater to $200 \mathrm{ft}$ below land surface in well 54BR. The vertical distribution of PFNA was identical to that of both PFOS and PFOA, indicating a common source, presumably the past discharge of AFFF at land surface.

\section{Sampling Method Comparison for PFASs}

The concentrations of PFASs in samples collected with the RCDM passive samplers were compared to those in samples from low-flow purging; samples were collected from the same depth in five wells, 11BR, 12S, 13S, 14S, and $28 \mathrm{~S}$ on the same day in 2017. 


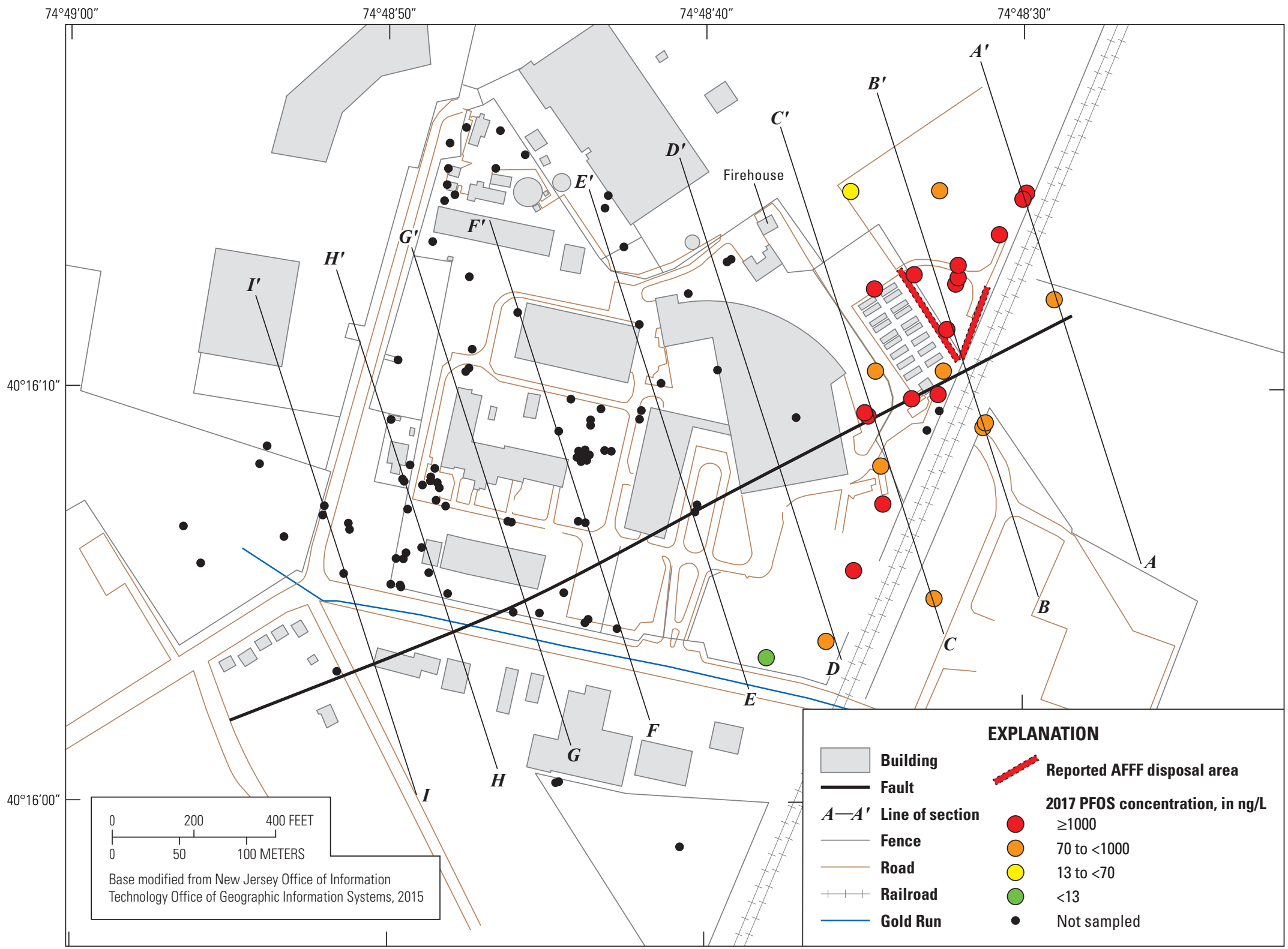

Figure 55. Areal extent of perfluorooctane sulfonate concentrations in wells at the former Naval Air Warfare Center, West Trenton, New Jersey, 2017. (PFOS, perfluorooctane sulfonate; AFFF, aqueous film-forming foam; ng/L, nanograms per liter) 


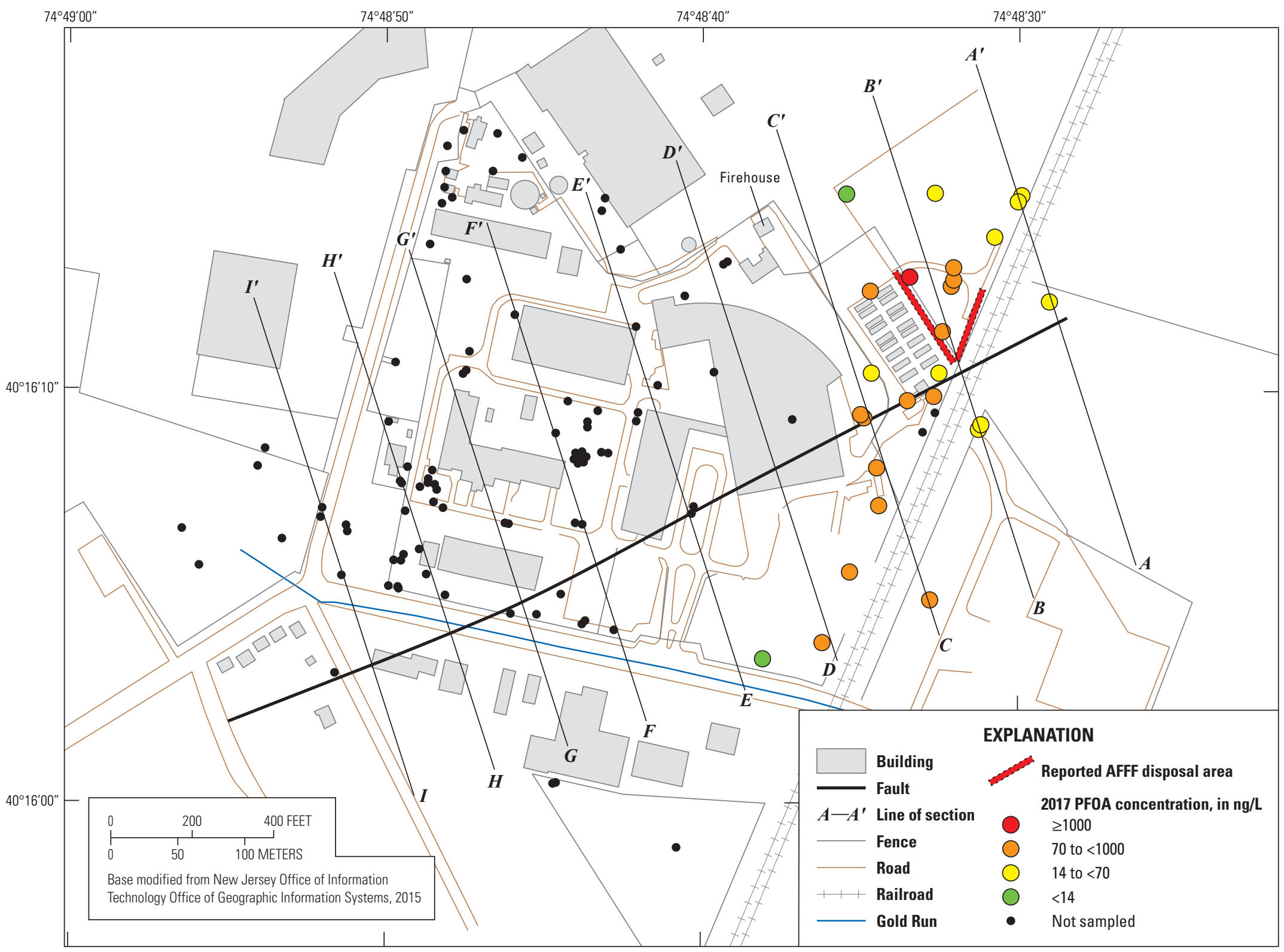

Figure 56. Areal extent of perfluorooctanoic acid concentrations in wells at the former Naval Air Warfare Center, West Trenton, New Jersey, 2017. (PFOA, perfluorooctanoic acid; AFFF, aqueous film-forming foam; ng/L, nanograms per liter) 


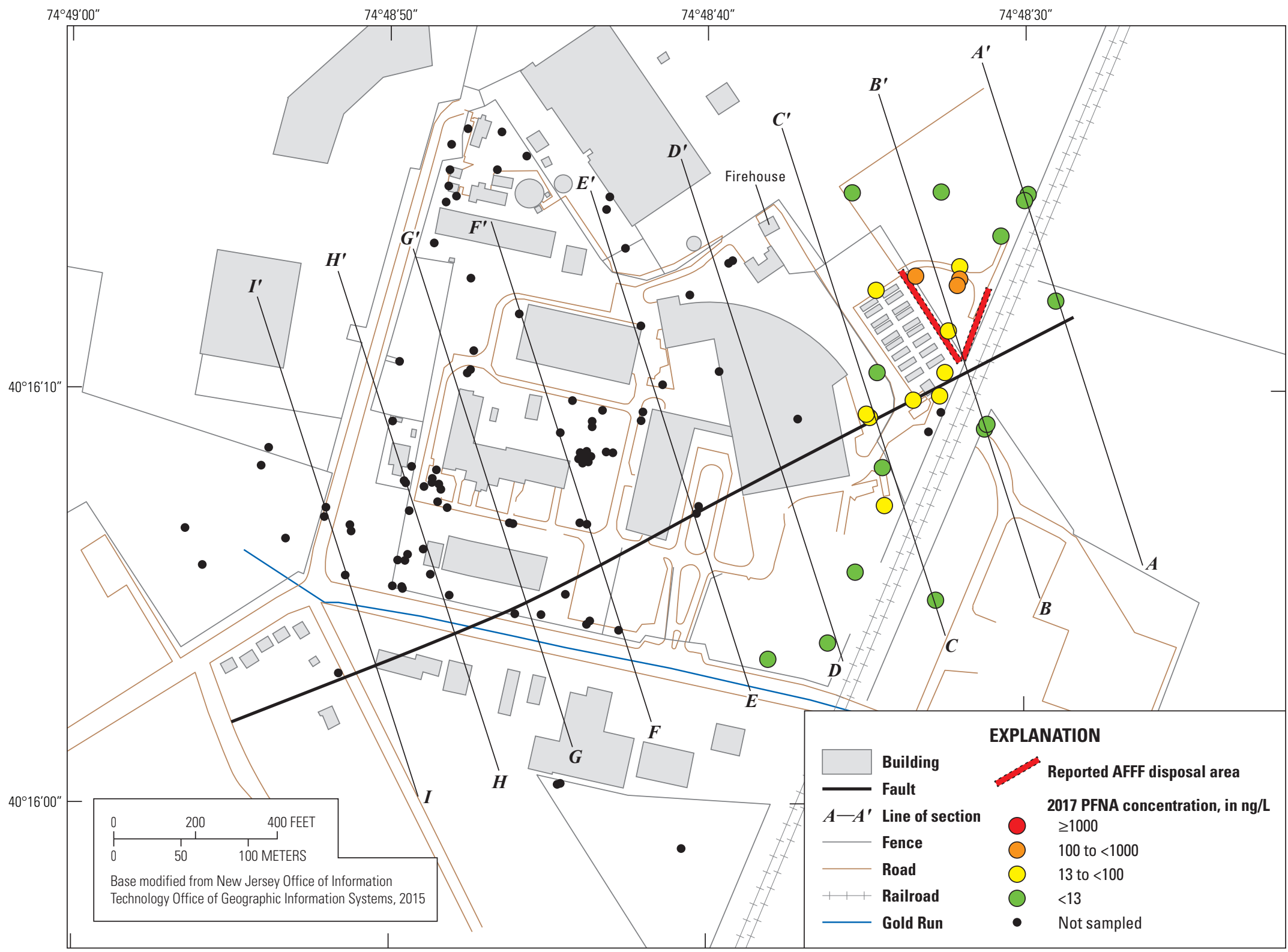

Figure 57. Areal extent of perfluorononanoic acid concentrations in wells at the former Naval Air Warfare Center, West Trenton, New Jersey, 2017. (PFNA, perfluorononanoic acid; AFFF, aqueous film-forming foam; ng/L, nanograms per liter) 


\section{A. 2015-17 PFOS maximum concentrations}

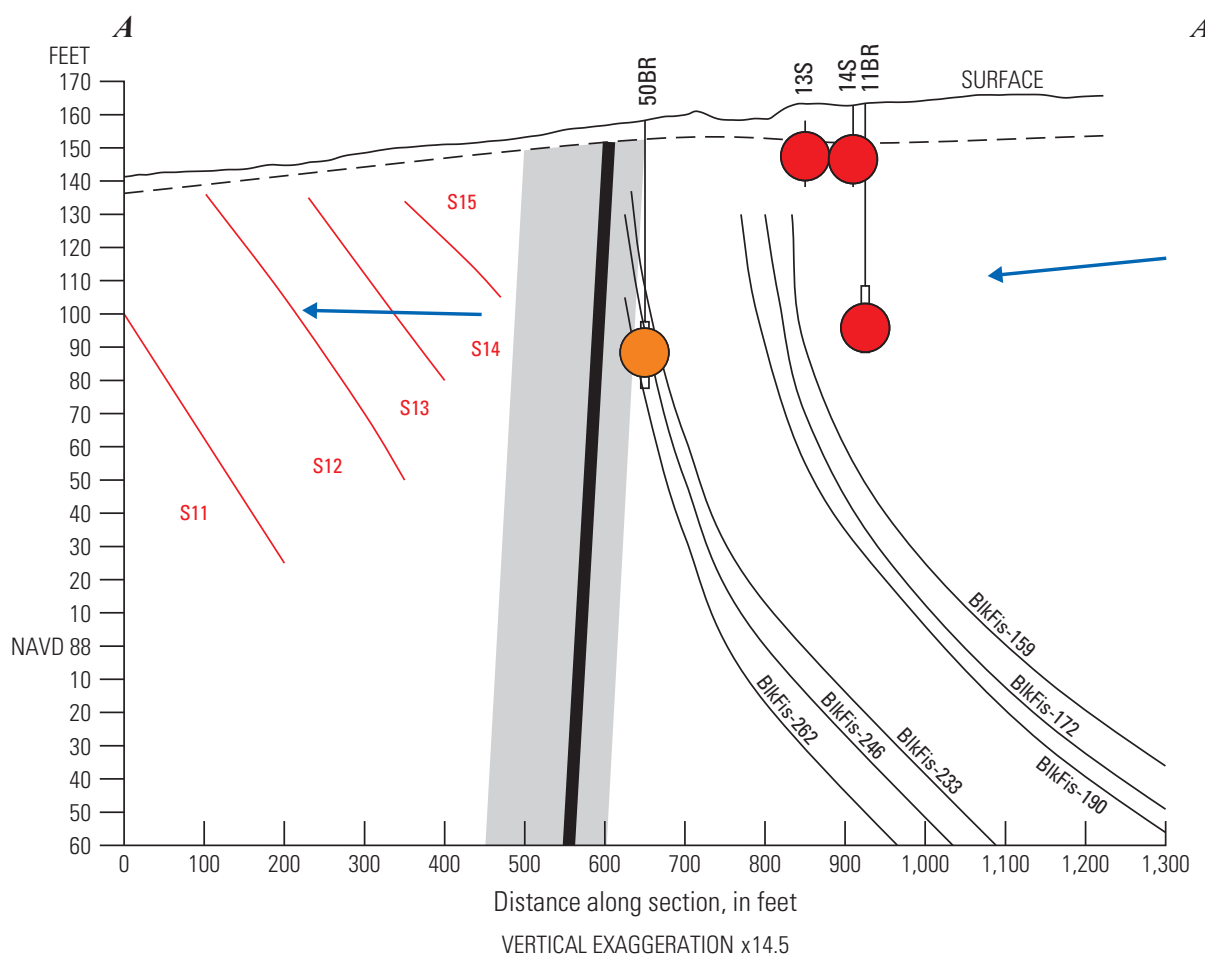

$A^{\prime}$

\section{B. 2015-17 PFOS maximum concentrations}

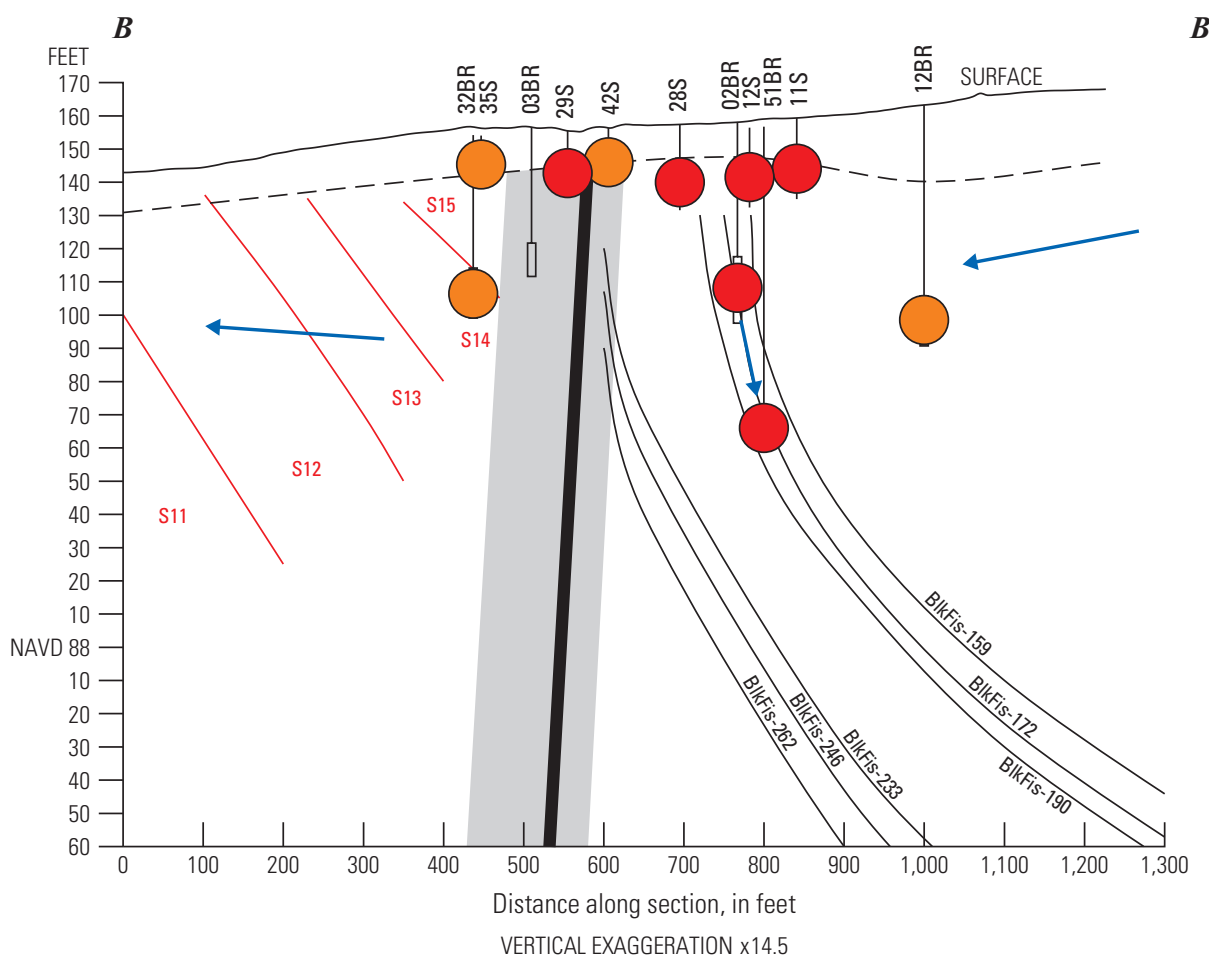

$B^{\prime}$

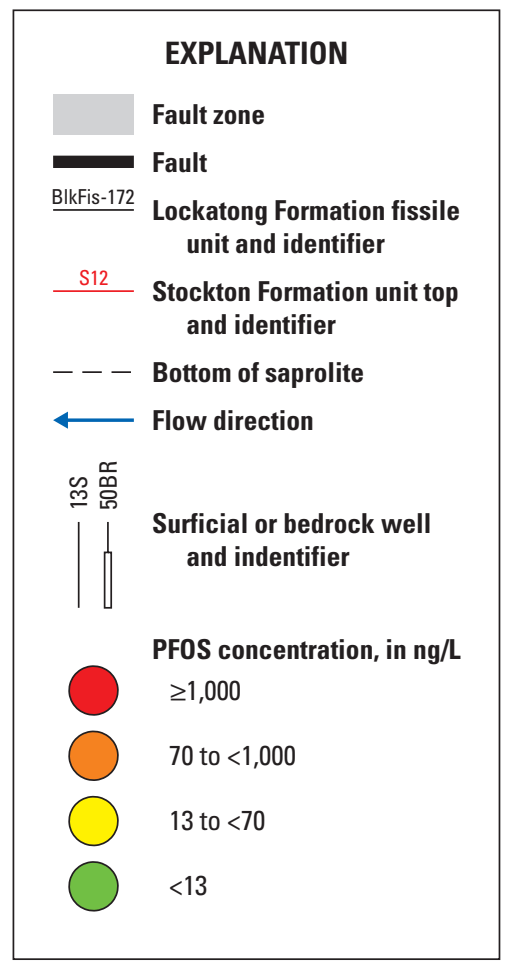

Figure 58. Vertical extent of maximum perfluorooctane sulfonate concentrations in wells along sections $A-A^{\prime}$ and $B-B^{\prime}$, former Naval Air Warfare Center, West Trenton, New Jersey, 2015-17. (PFOS, perfluorooctane sulfonate; ng/L, nanograms per liter) 


\section{A. 2015-2017 PFOS maximum concentrations}

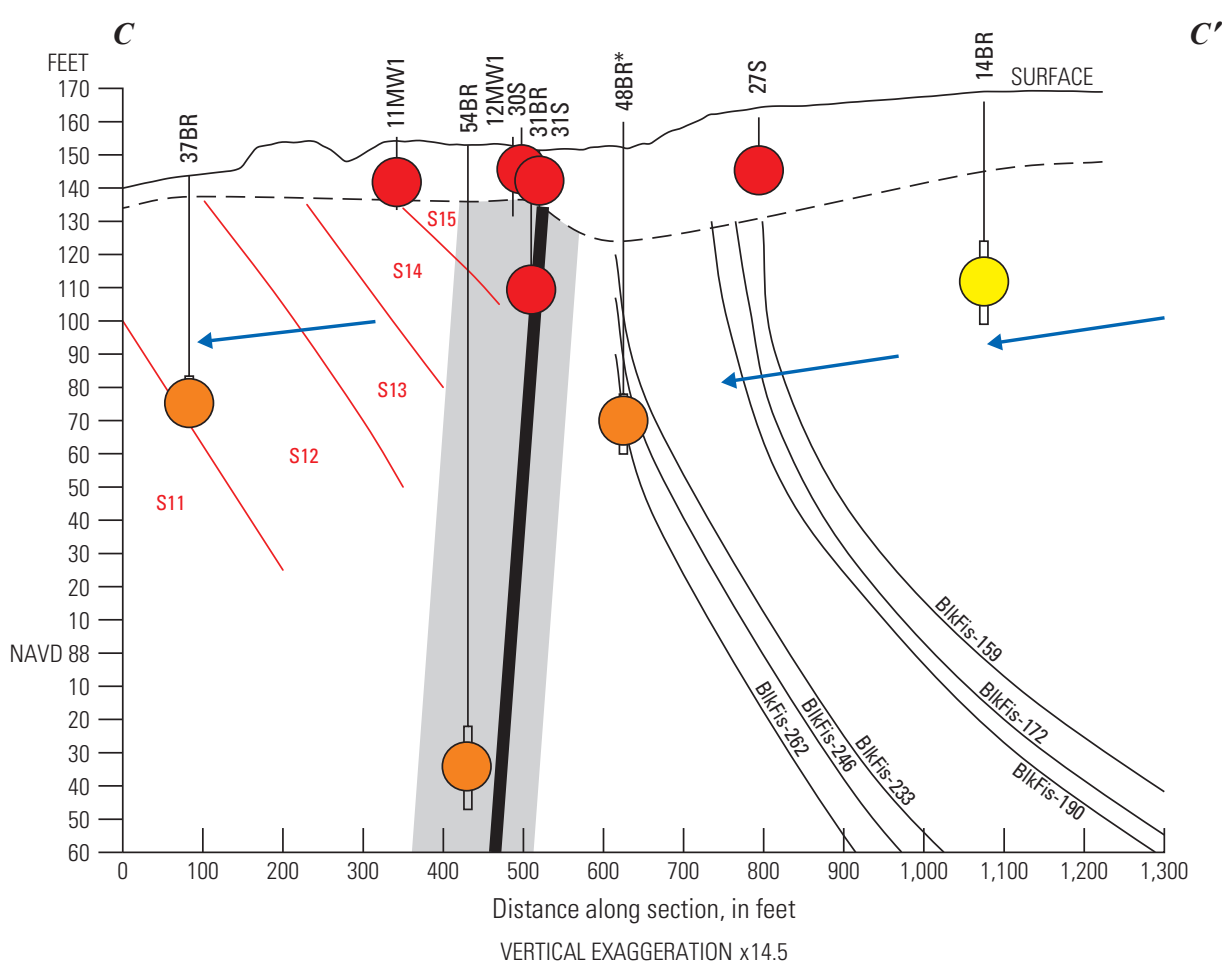

\section{B. 2015-17 PFOS maximum concentrations}

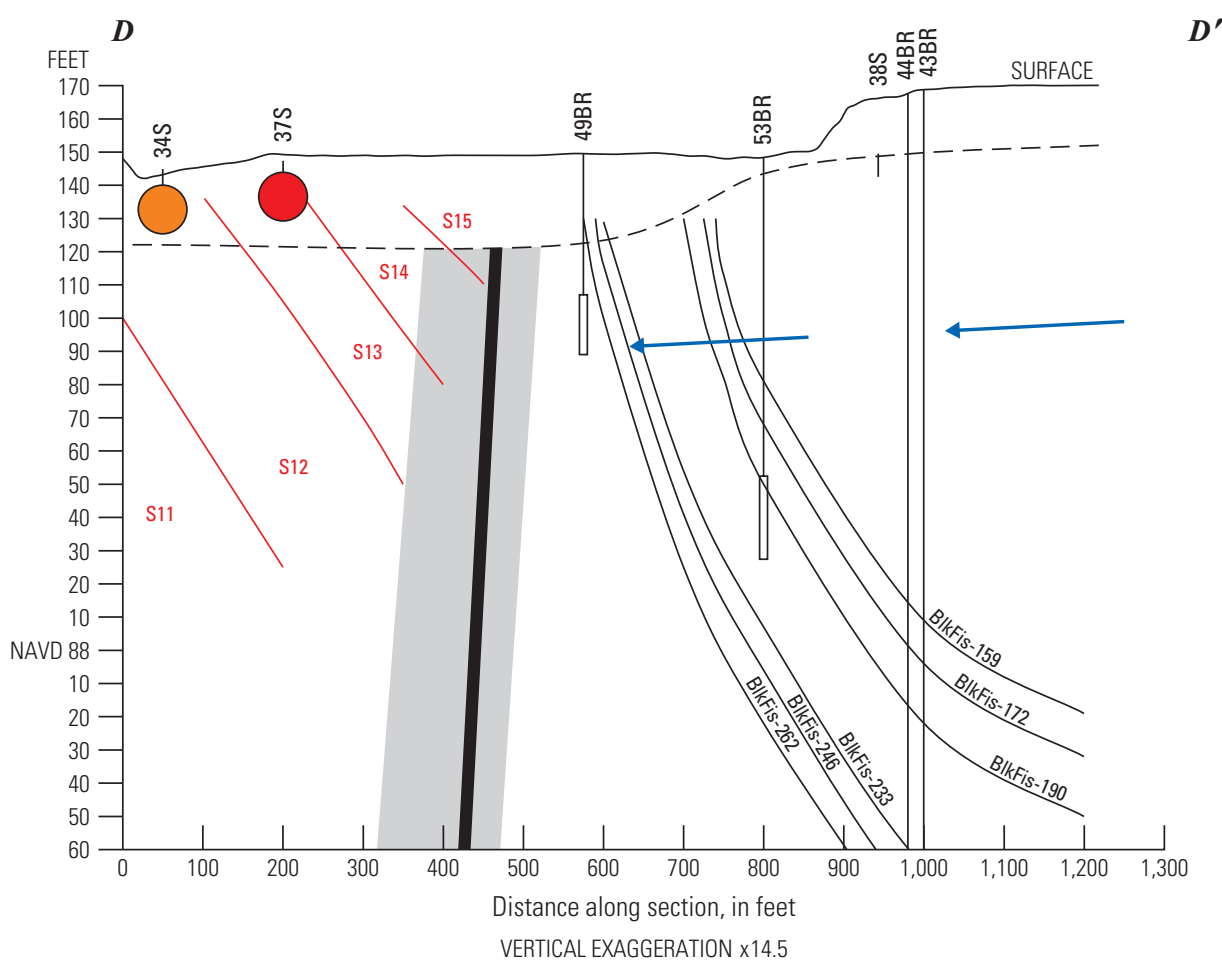

\begin{tabular}{|c|c|}
\hline & EXPLANATION \\
\hline & Fault zone \\
\hline & Fault \\
\hline & $\begin{array}{l}\text { Lockatong Formation fissile } \\
\text { unit and identifier }\end{array}$ \\
\hline S12 & $\begin{array}{l}\text { Stockton Formation unit top } \\
\text { and identifier }\end{array}$ \\
\hline & Bottom of saprolite \\
\hline & Flow direction \\
\hline & $\begin{array}{l}\text { Surficial or bedrock well } \\
\text { and indentifier }\end{array}$ \\
\hline & PFOS concentration, in ng/L \\
\hline & $\geq 1,000$ \\
\hline & 70 to $<1,000$ \\
\hline & 13 to $<70$ \\
\hline & $<13$ \\
\hline
\end{tabular}

Figure 59. Vertical extent of perfluorooctane sulfonate concentrations in wells along sections $C-C^{\prime}$ and $D-D^{\prime}$, former Naval Air Warfare Center, New Jersey, 2015-17. (PFOS, perfluorooctane sulfonate; ng/L, nanograms per liter) 


\section{A. 2015-17 PFOA maximum concentrations}

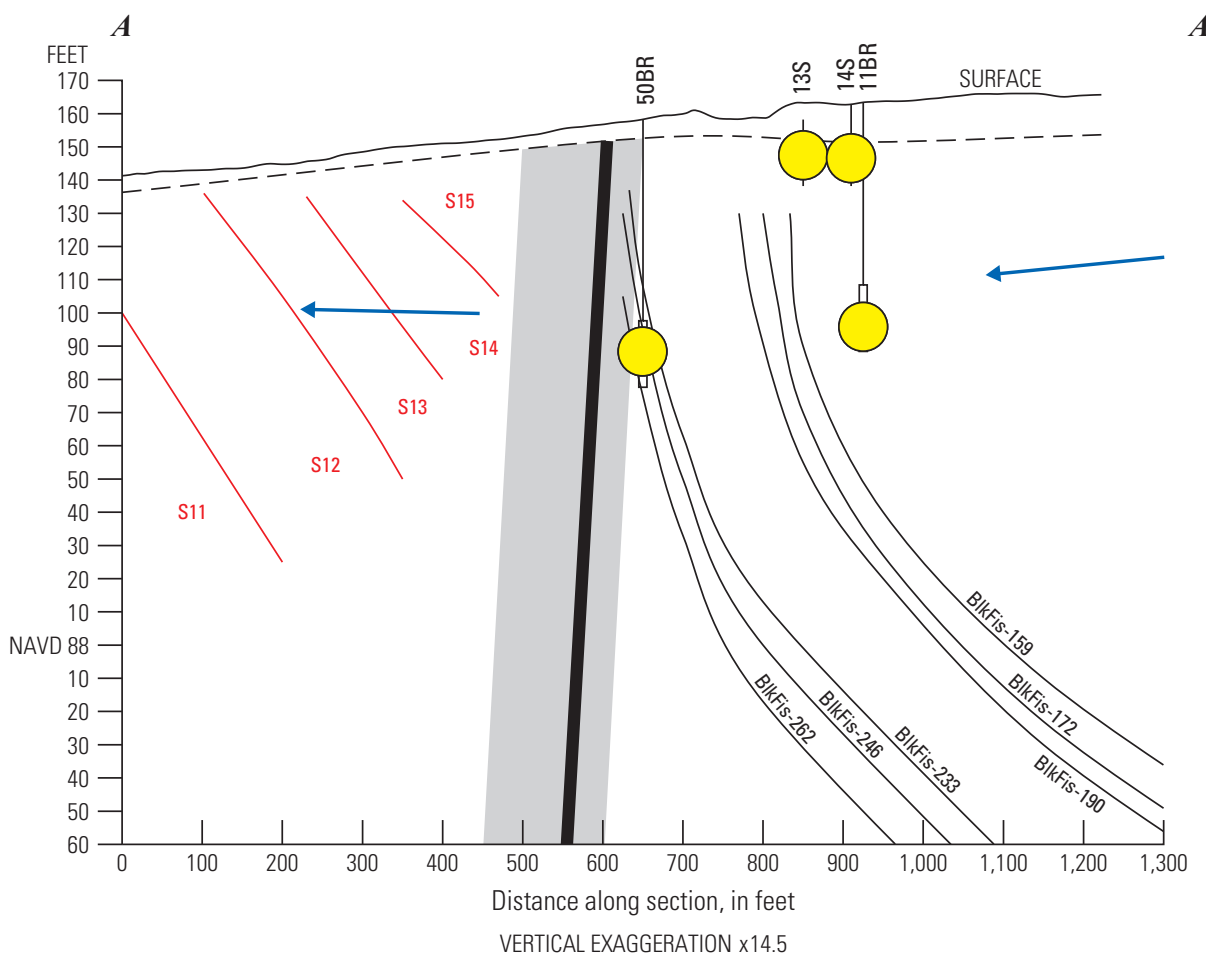

\section{B. 2015-17 PF0A maximum concentrations}

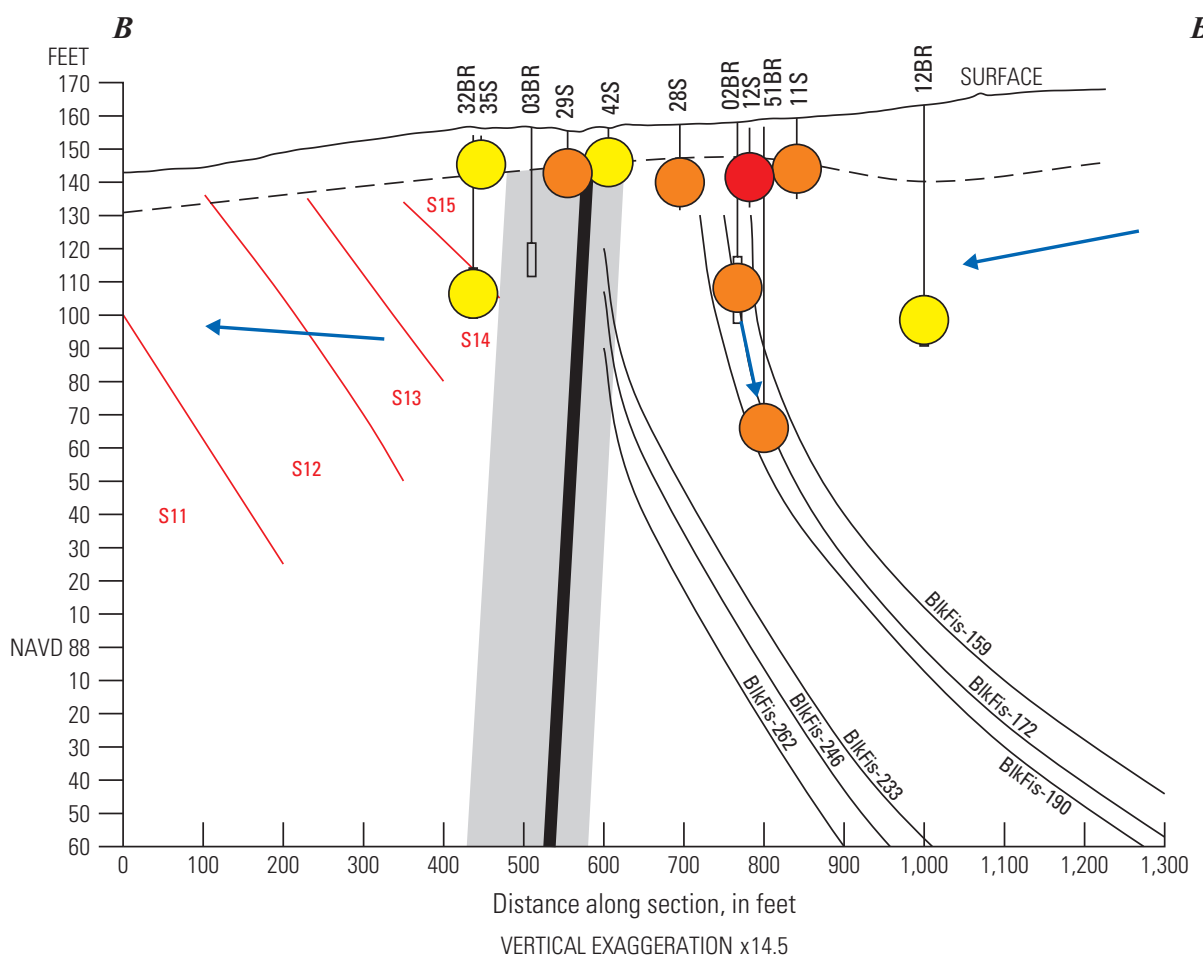

$B^{\prime}$

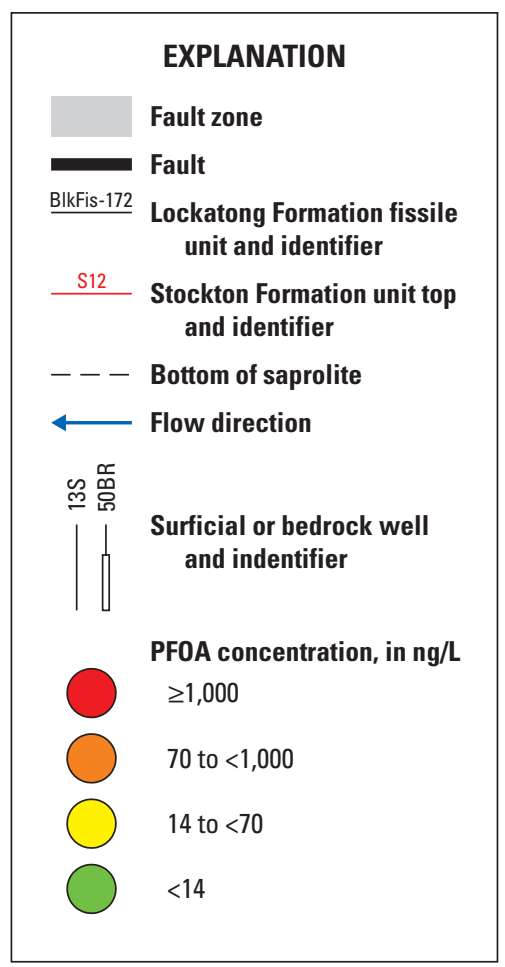

Figure 60. Vertical extent of perfluorooctanoic acid concentrations in wells along sections $A-A^{\prime}$ and B-B', former Naval Air Warfare Center, West Trenton, New Jersey, 2015-17. (PFOA, perfluorooctanoic acid; ng/L, nanograms per liter) 


\section{A. 2015-2017 PFOA maximum concentrations}

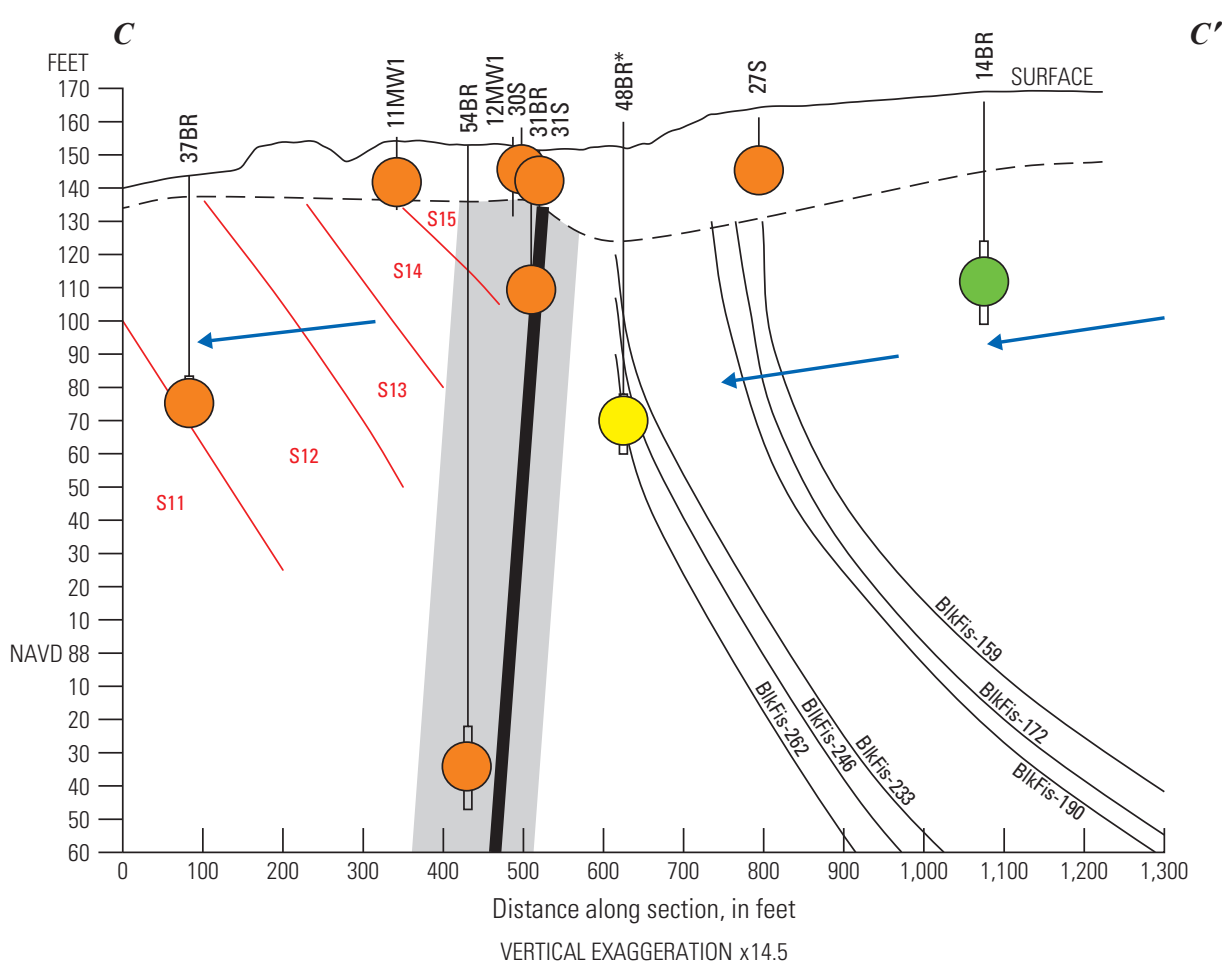

\section{B. 2015-17 PF0A maximum concentrations}

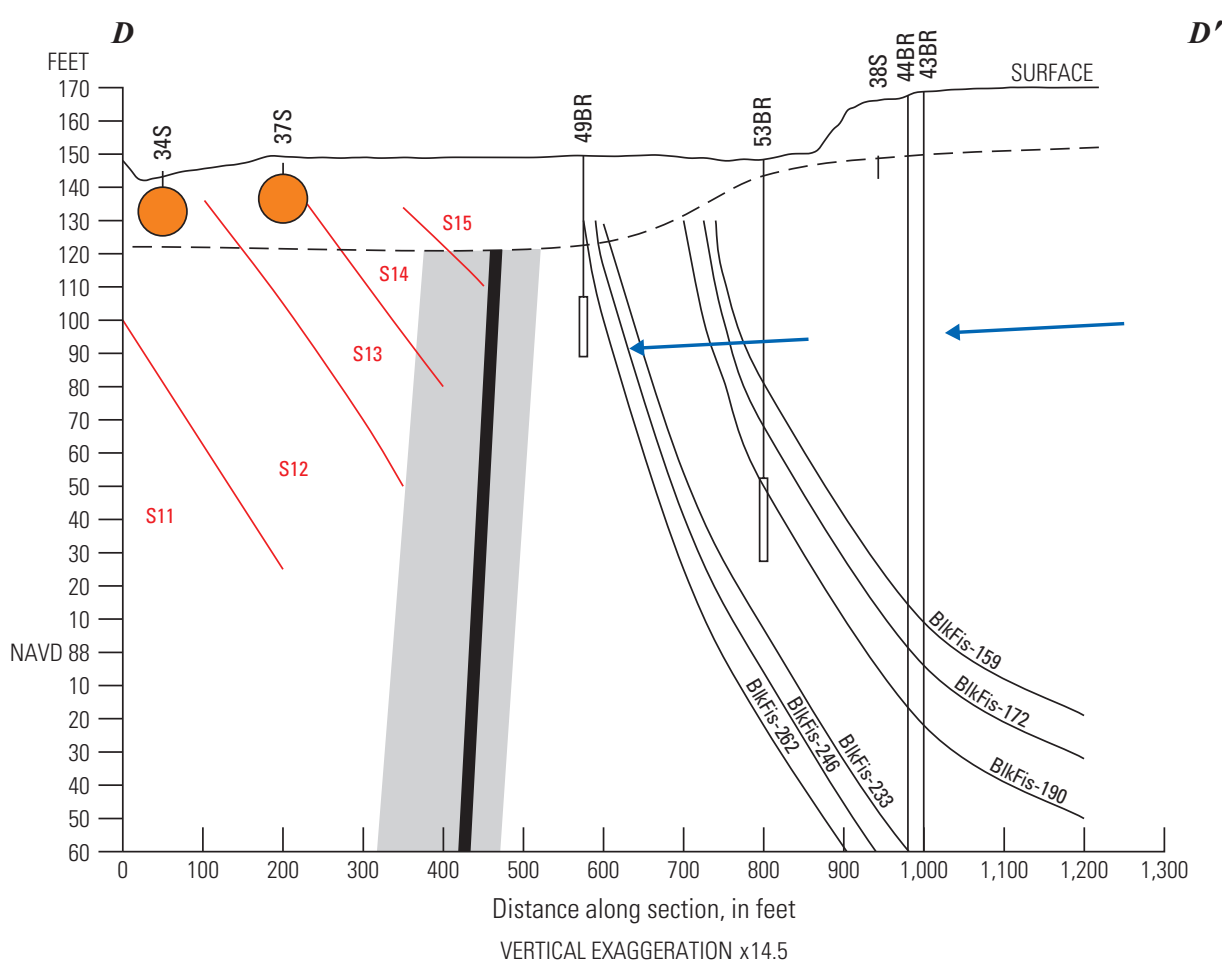

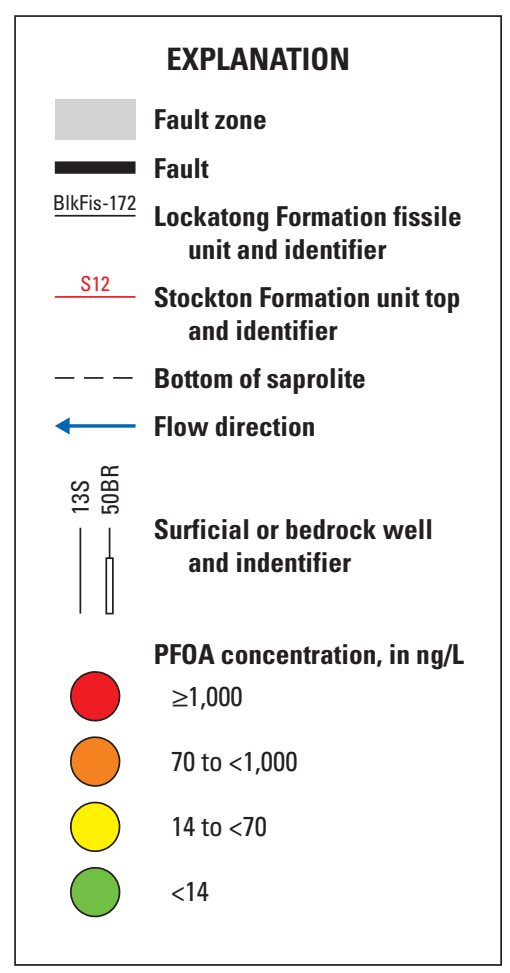

Figure 61. Vertical extent of perfluorooctanoic acid concentrations in wells along sections $C-C^{\prime}$ and $D-D^{\prime}$, former Naval Air Warfare Center, West Trenton, New Jersey, 2015-17. (PFOA, perfluorooctanoic acid; ng/L, nanograms per liter) 


\section{A. 2015-17 PFNA maximum concentrations}

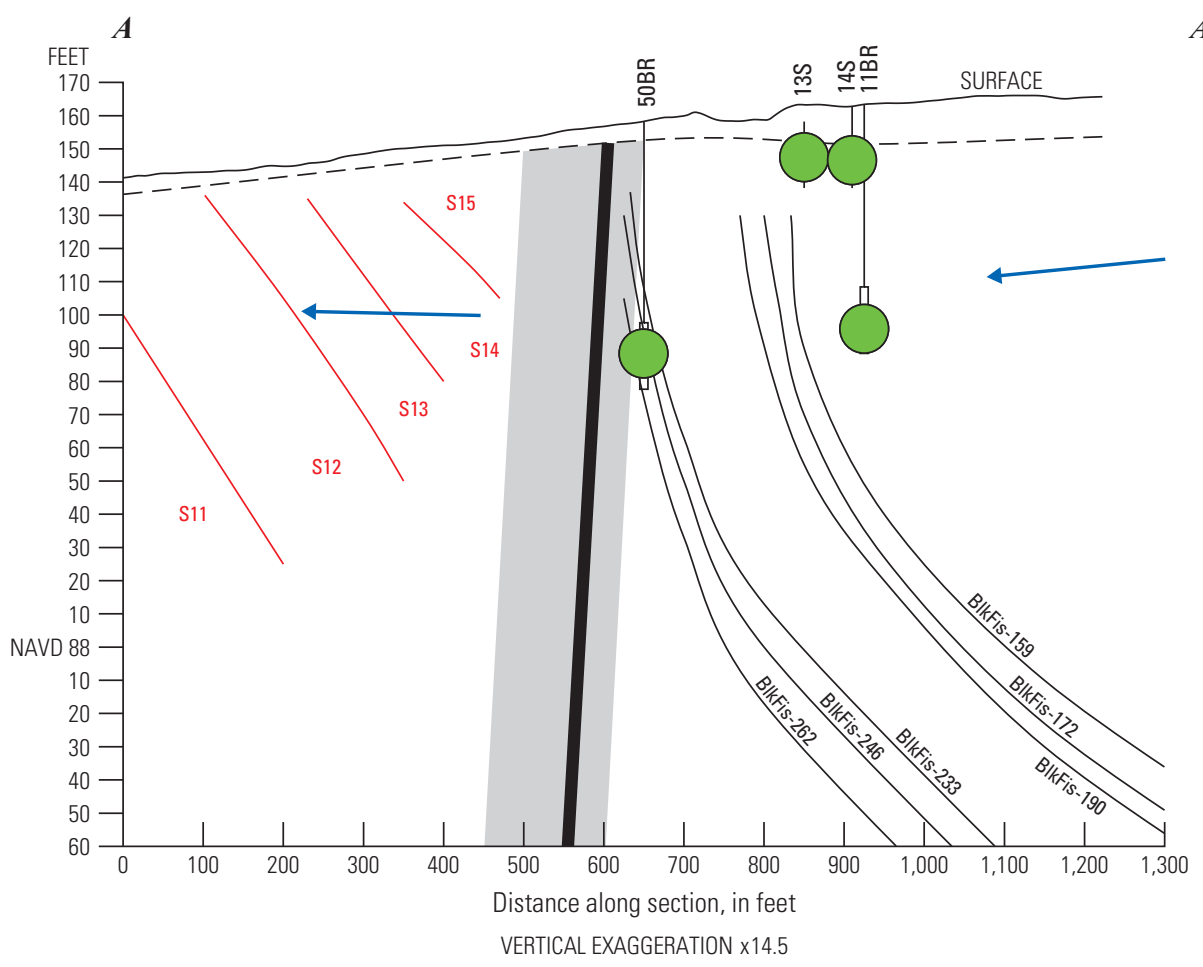

$A^{\prime}$

\section{B. 2015-17 PFNA maximum concentrations}

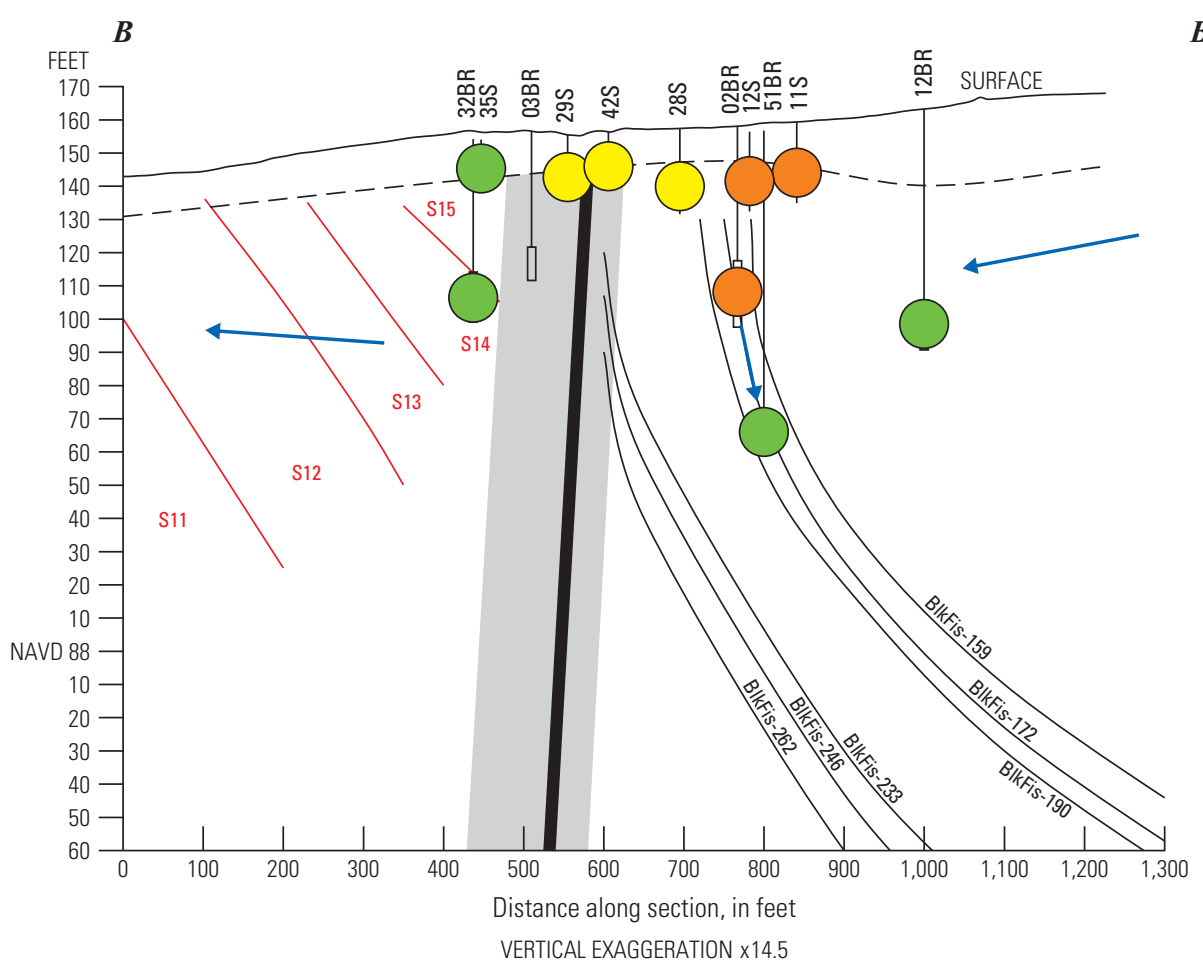

$\boldsymbol{B}^{\prime}$

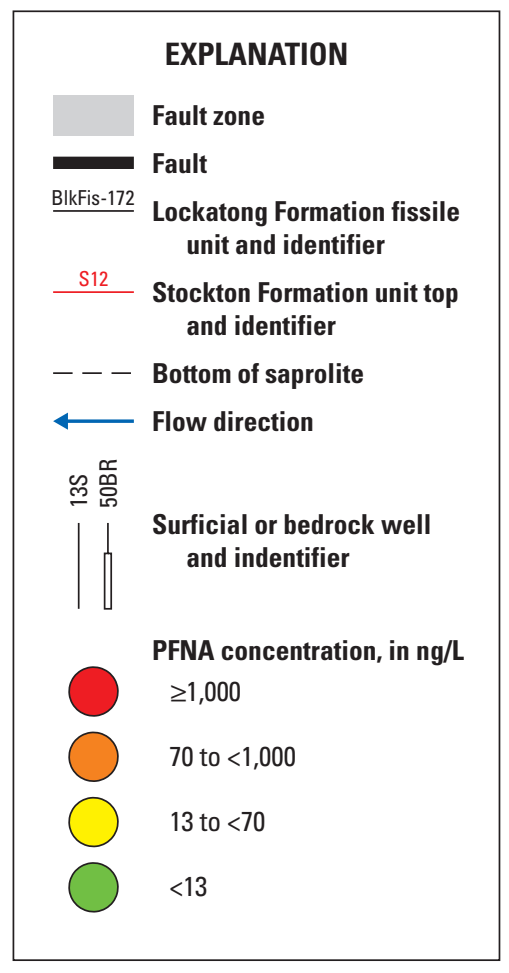

Figure 62. Vertical extent of perfluorononanoic acid concentrations in wells along sections $A-A^{\prime}$ and B-B', former Naval Air Warfare Center, West Trenton, New Jersey, 2015-17. (PFNA, perfluorononanoic acid; ng/L, nanograms per liter) 


\section{A. 2015-2017 PFNA maximum concentrations}

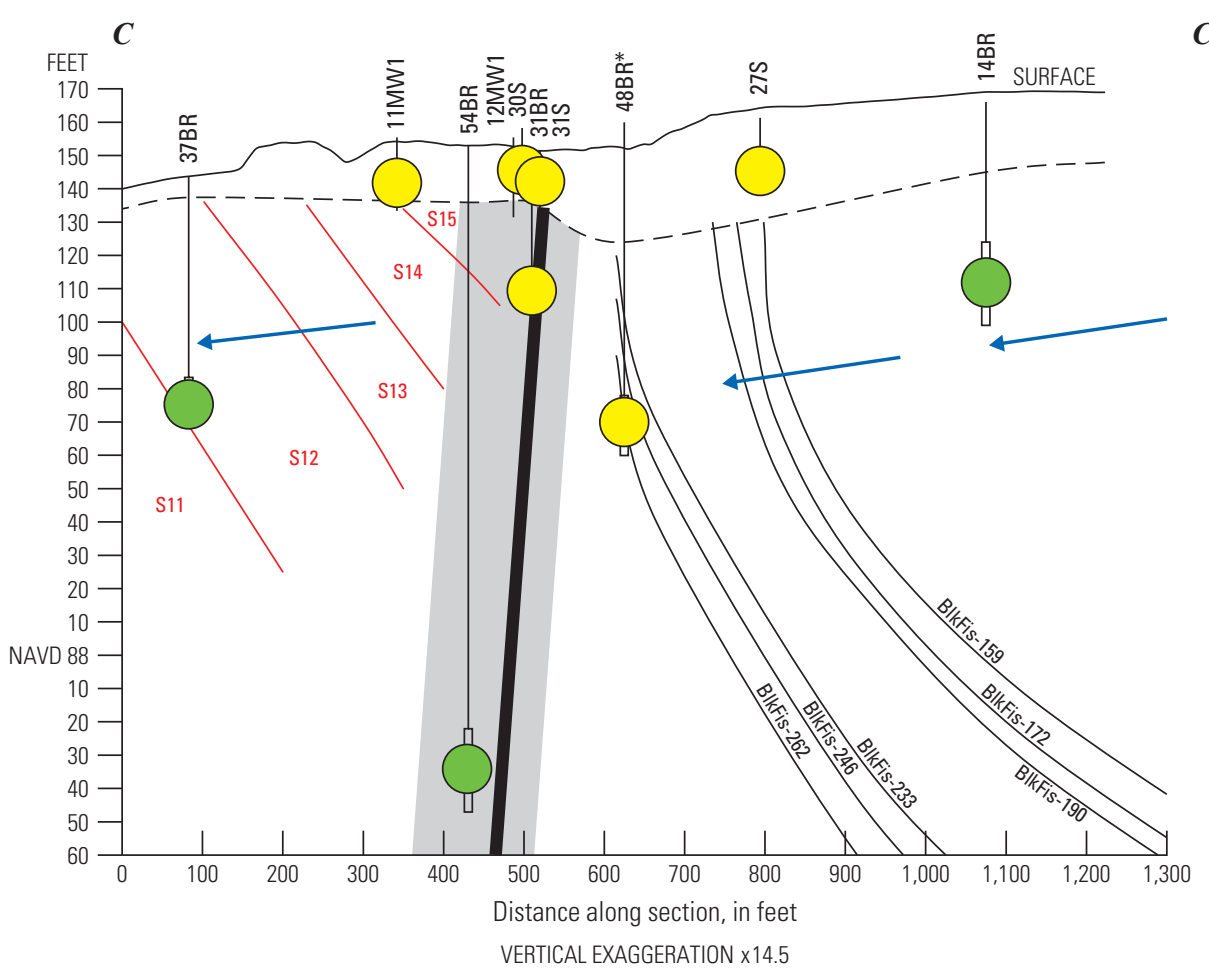

\section{B. 2015-17 PFNA maximum concentrations}

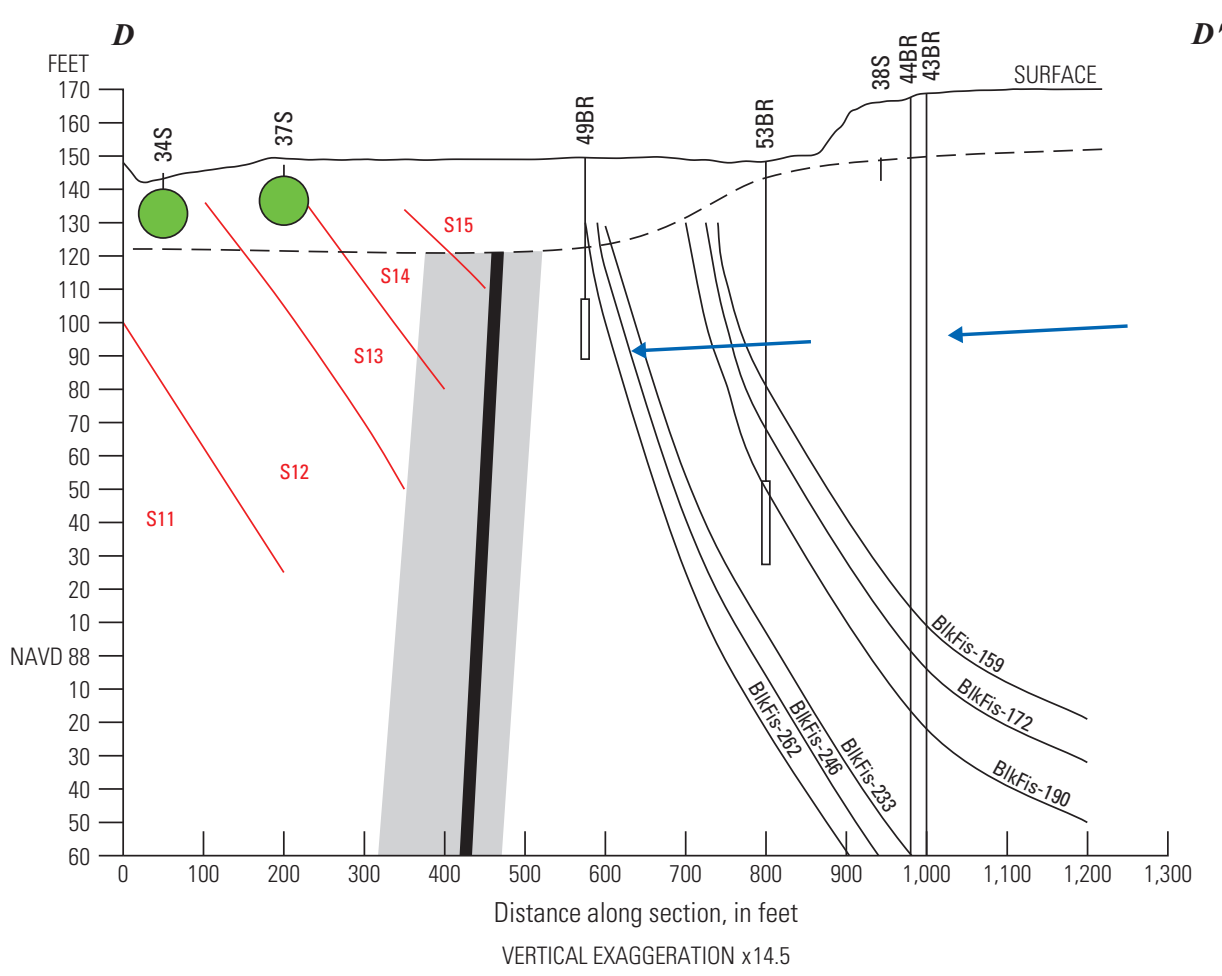

\begin{tabular}{|c|c|}
\hline & EXPLANATION \\
\hline & Fault zone \\
\hline & Fault \\
\hline BlkFis-172 & $\begin{array}{l}\text { Lockatong Formation fissile } \\
\text { unit and identifier }\end{array}$ \\
\hline S12 & $\begin{array}{l}\text { Stockton Formation unit top } \\
\text { and identifier }\end{array}$ \\
\hline & Bottom of saprolite \\
\hline & Flow direction \\
\hline & $\begin{array}{l}\text { Surficial or bedrock well } \\
\text { and indentifier }\end{array}$ \\
\hline & $\begin{array}{l}\text { PFNA concentration, in } \mathbf{n g} / \mathbf{L} \\
\geq 1,000\end{array}$ \\
\hline & 70 to $<1,000$ \\
\hline & 13 to $<70$ \\
\hline & $<13$ \\
\hline
\end{tabular}

Figure 63. Vertical extent of perfluorononanoic acid concentrations in wells along sections $C-C^{\prime}$ and $D-D$ ', former Naval Air Warfare Center, West Trenton, New Jersey, 2015-17. (PFNA, perfluorononanoic acid; ng/L, nanograms per liter) 
Concentrations of PFASs in samples from these five wells and two blanks are shown in figures 64,65 , and 66 . The concentration of PFOS, PFOA, and PFNA in samples collected with an RCDM sampler are plotted on the $\mathrm{X}$ axis, and the concentrations in samples collected by low-flow purging are plotted on the $\mathrm{Y}$ axis. If concentrations from both sampling methods are equal, the points will fall on or close to the 1:1 correspondence line. The results for each of the three PFAS compounds show excellent agreement between the two sampling methods. Although additional testing is required to confirm these findings for a range of PFAS concentrations in different groundwater settings, these preliminary findings indicate that RCDM passive samplers can collect PFAS samples equivalent to those collected by low-flow purging at the NAWC site.

\section{Areal Extent of 1,4-dioxane}

Wells at the former NAWC site were sampled and analyzed for 1,4-dioxane, a contaminant frequently present at sites with chlorinated solvent contamination. 1,4-dioxane was used as an anti-corrosion agent in containers of 1,1,1-trichloroethane, which was known to have been used at NAWC after the use of TCE was discontinued. All 18 wells sampled for 1,4-dioxane were found to contain less than the NJDEP MCL of $<0.4 \mu \mathrm{g} / \mathrm{L}$ in 2017 (fig. 67).

\section{Summary and Conclusions}

In a study conducted by the U.S. Geological Survey in cooperation with the U.S. Navy, samples were collected from groundwater wells and analyzed for volatile organic compounds (VOCs) and per- and polyfluoroalkyl substances (PFASs) at the former Naval Air Warfare Center (NAWC) site in West Trenton, New Jersey, from 2014 to 2017. Trichloroethene (TCE) was used as a heat exchange liquid in cooling systems at NAWC from the mid-1950s to early 1990s. TCE from this system leaked onto the ground and into the fractured rock aquifer beneath the NAWC site. Aqueous filmforming foam (AFFF) was discharged to the ground in the northeastern part of the NAWC site around 1990. As a result, PFASs from the AFFF seeped into the fractured rock aquifer beneath this area of the NAWC site.

VOC data from samples collected from wells at the NAWC site were evaluated areally and vertically by plotting them on maps and cross sections. The areas of VOC contamination decreased slightly on the northwestern and northeastern sides of the NAWC site from 2014 to 2017 under the influence of the pump-and-treat system, natural attenuation processes, and the bioaugmentation studies that were conducted at the site. cis-1,2-dichloroethene (cisDCE) and vinyl chloride (VC), the biodegradation byproducts of TCE, were present only in wells that had been originally contaminated with TCE. Higher concentrations of all three VOCs were present in the western
Site 1 area compared to the eastern Site 3 area of the NAWC site. Most of the wells with the highest VOC concentrations were in the area between and around Buildings 40 and 41 where most of the original TCE was spilled or leaked while the base was in operation. TCE, cisDCE, and VC were not found in offsite wells to the south, west, and north of the NAWC site. TCE and cisDCE were found at low concentrations (TCE, $3.3 \mathrm{mg} / \mathrm{L}$; cisDCE $2.2 \mathrm{mg} / \mathrm{L}$ ) in one well (50BR) to the east of the current NAWC site boundary in 2017. These findings indicate that the pump-and-treat system was continuing to contain the VOC contamination on the western side of the NAWC property but not on the eastern side of the facility.

Vertically, TCE, cisDCE, and VC contamination was found to extend from shallow overburden wells to bedrock wells that are more than $200 \mathrm{ft}$ deep in the Site 1 and the Site 3 areas of the site. The highest TCE concentrations were present in wells between 10 and $210 \mathrm{ft}$ deep in the area around and between Buildings 40 and 41 where most of the TCE had spilled or leaked in the past. The highest cisDCE concentrations tended to be present in wells between 10 and $165 \mathrm{ft}$ deep, mostly in the area of the 2008 bioaugmentation study and in the area between Buildings 40 and 41 . The highest VC concentrations were present in wells 25 to $100 \mathrm{ft}$ deep in the 2008 bioaugmentation study area and in in the southwest corner of the NAWC property where a past jet fuel spill stimulated indigenous dechlorinating bacteria to degrade TCE.

Most wells at NAWC had stable or gradually downward trends in VOC concentrations over time since the base activities ceased in 1999. VOC concentrations in most wells had a similar stable or gradually downward trend from 2014 to 2017. Extraction wells 29BR, 45BR, and BRP02 were exceptions. These wells have apparently started drawing in more contaminated groundwater than they had previously.

Several wells showed the effects of the two bioaugmentation studies in 2005 and 2008 on VOC concentrations, particularly the injection wells. All injection wells in the 2005 bioaugmentation study (BRP01, 16BR, 38BR, and 41BR) had dramatic decreases in TCE concentrations within 6 months of the injection of bacteria and food. Concentrations of cisDCE and $\mathrm{VC}$ frequently also decreased to much lower levels within 6 months, but inevitably these two compounds rebounded after a couple of years to slightly less than their original concentrations. TCE concentrations remained low in all these wells through 2017.

Well 36BR, the injection well for the 2008 bioaugmentation study, had an immediate and dramatic decrease in TCE concentrations within 2 months of injection. Concentrations of cisDCE and VC increased in proportion to the TCE decrease within 6 months and 1 year, respectively. Concentrations of cisDCE and VC remained higher than they were before the injection, but approximately equal from 2011 to 2017. The persistence of higher cisDCE and VC concentrations is an indication that TCE biodegradation is still occurring in well 36BR, but that it may stalled rather than going to completion. 


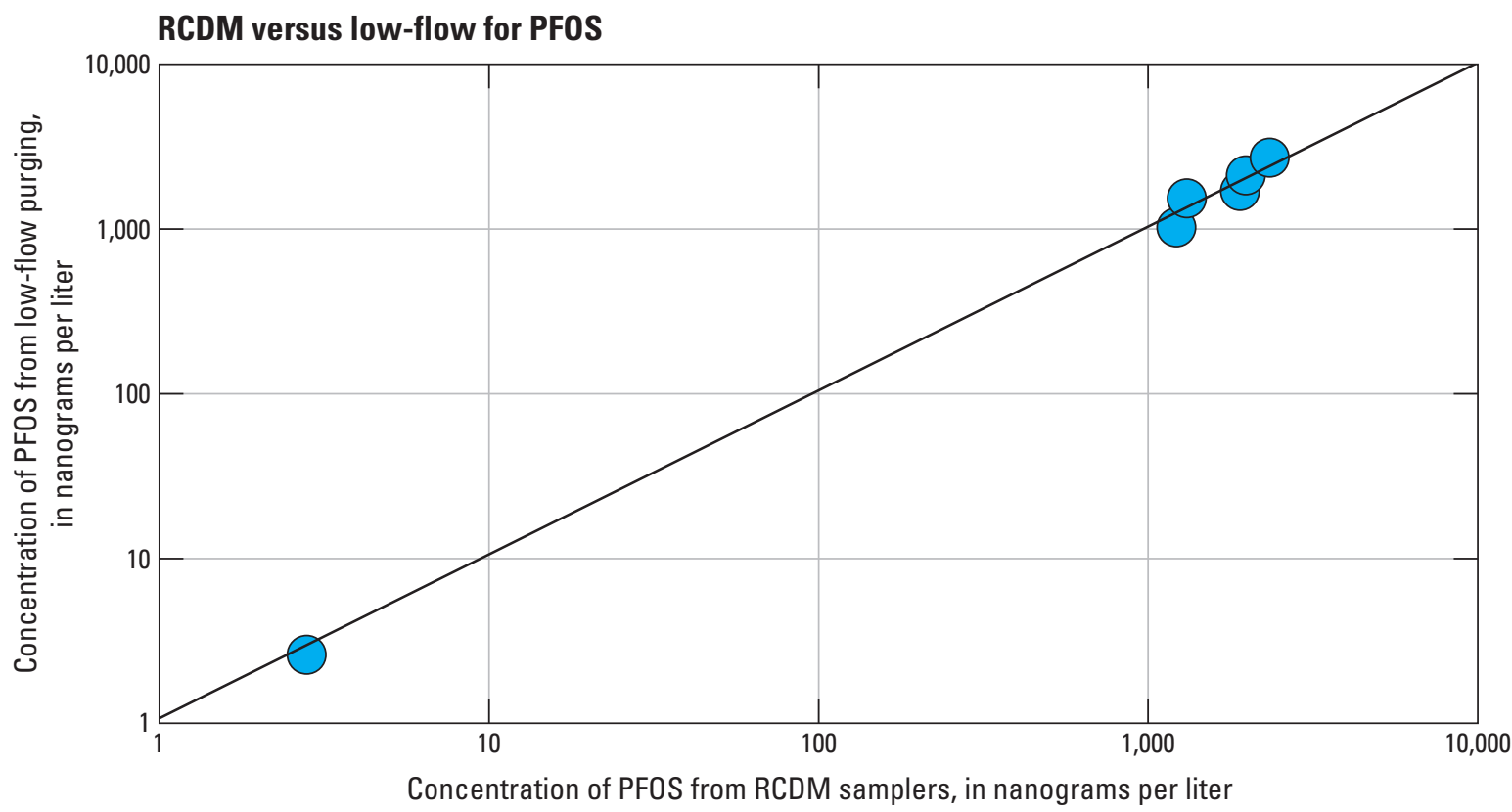

Figure 64. Concentrations of perfluorooctane sulfonate (PFOS) in samples collected using regenerated cellulose dialysis membrane samplers in relation to concentrations of PFOS from low-flow purging, with a 1:1 linear relation line. (PFOS, perfluorooctane sulfonate; RCDM, regenerated cellulose dialysis membrane; $\mathrm{ng} / \mathrm{L}$, nanograms per liter)

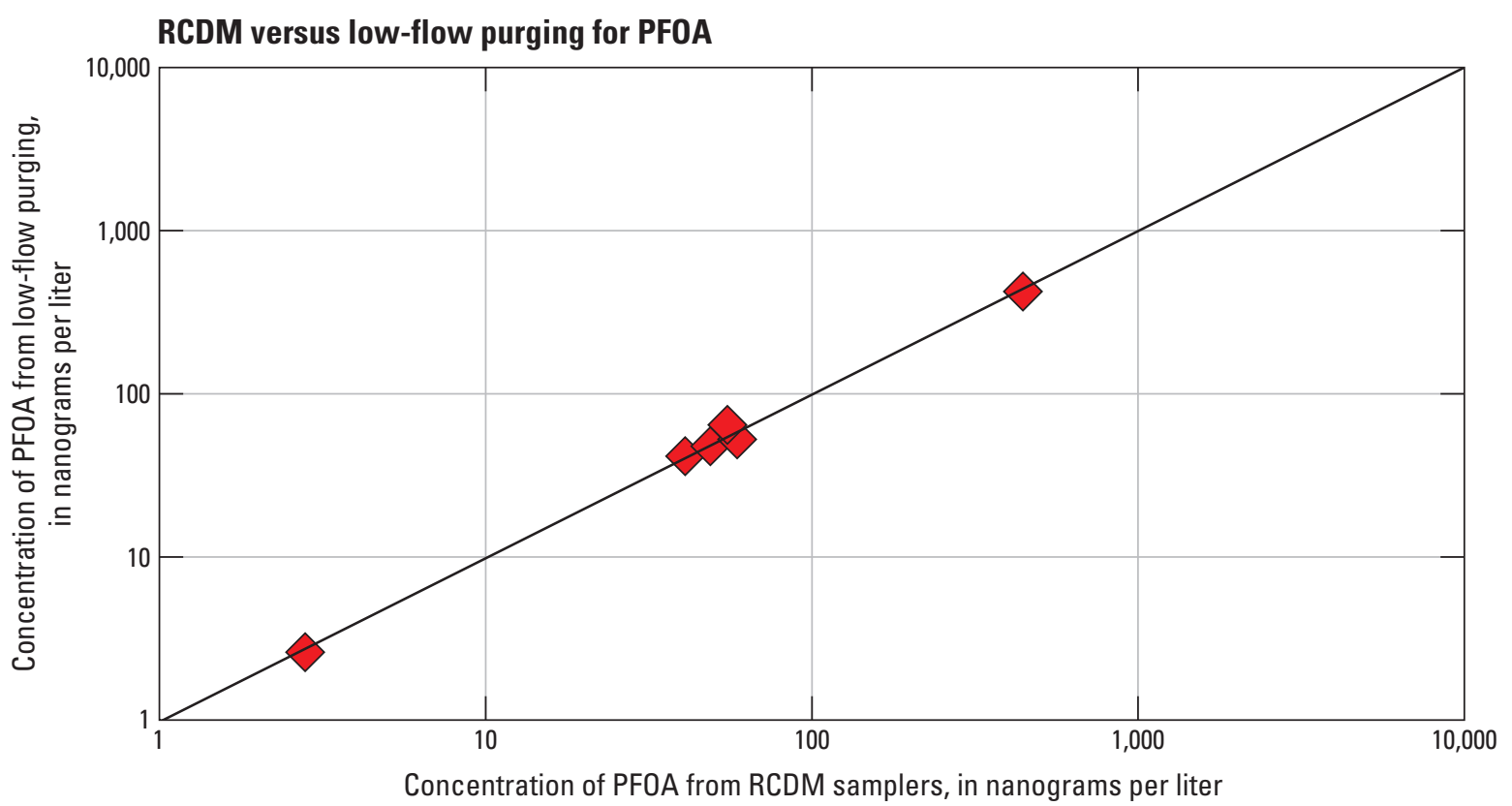

Figure 65. Concentrations of perfluorooctanoic acid (PFOA) in samples collected using regenerated cellulose dialysis membrane samplers in relation to concentrations of PFOA from low-flow purging, with a 1:1 linear relation line. (PFOA, perfluorooctanoic acid; RCDM, regenerated cellulose dialysis membrane; $\mathrm{ng} / \mathrm{L}$, nanograms per liter) 


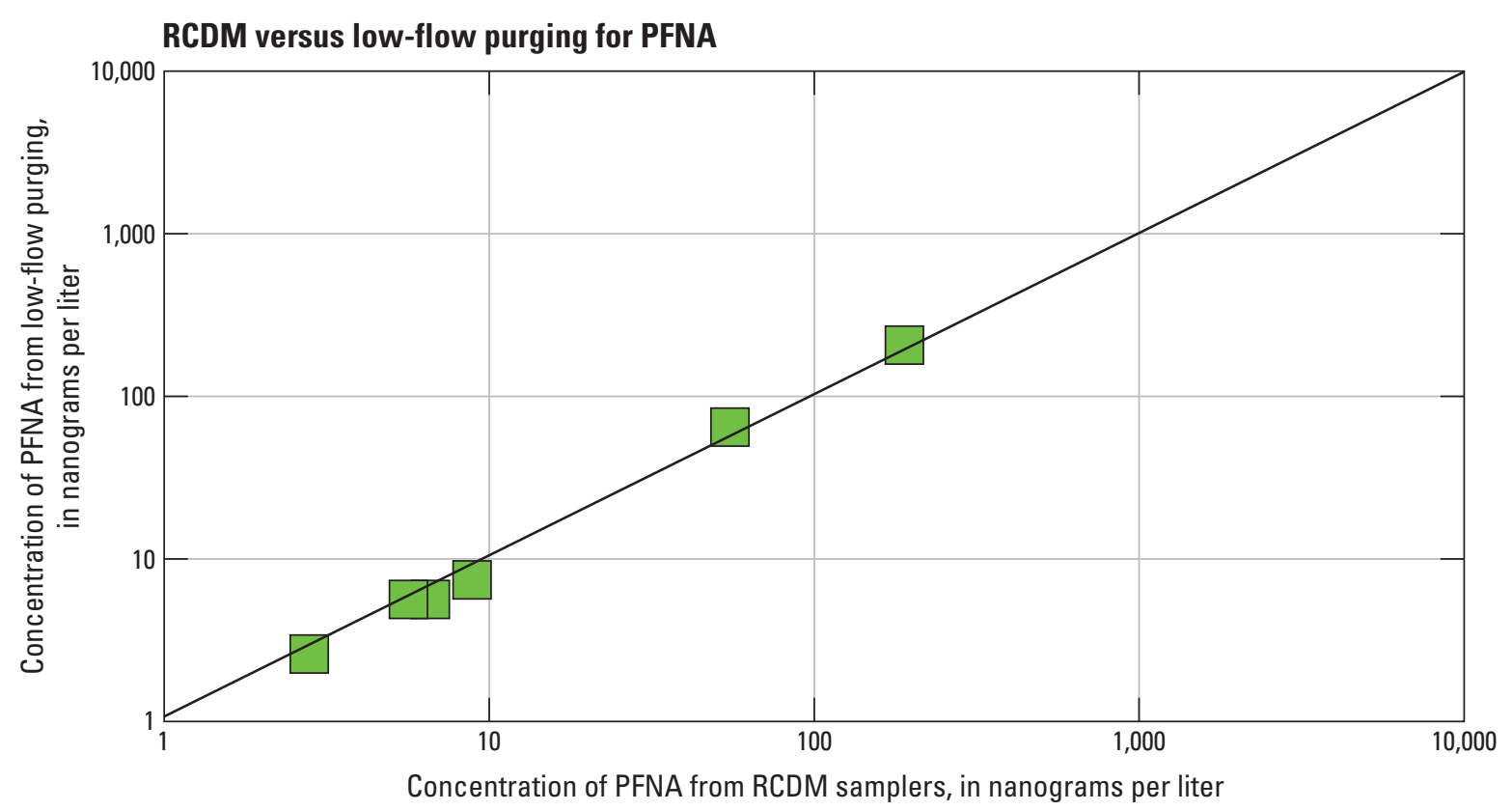

Figure 66. Concentrations of perfluorononanoic acid (PFNA) in samples collected using regenerated cellulose dialysis membrane samplers in relation to concentrations of PFNA from low-flow purging, with a 1:1 linear relation line. (PFNA, perfluorononanoic acid; RCDM, regenerated cellulose dialysis membrane; ng/L, nanograms per liter) 


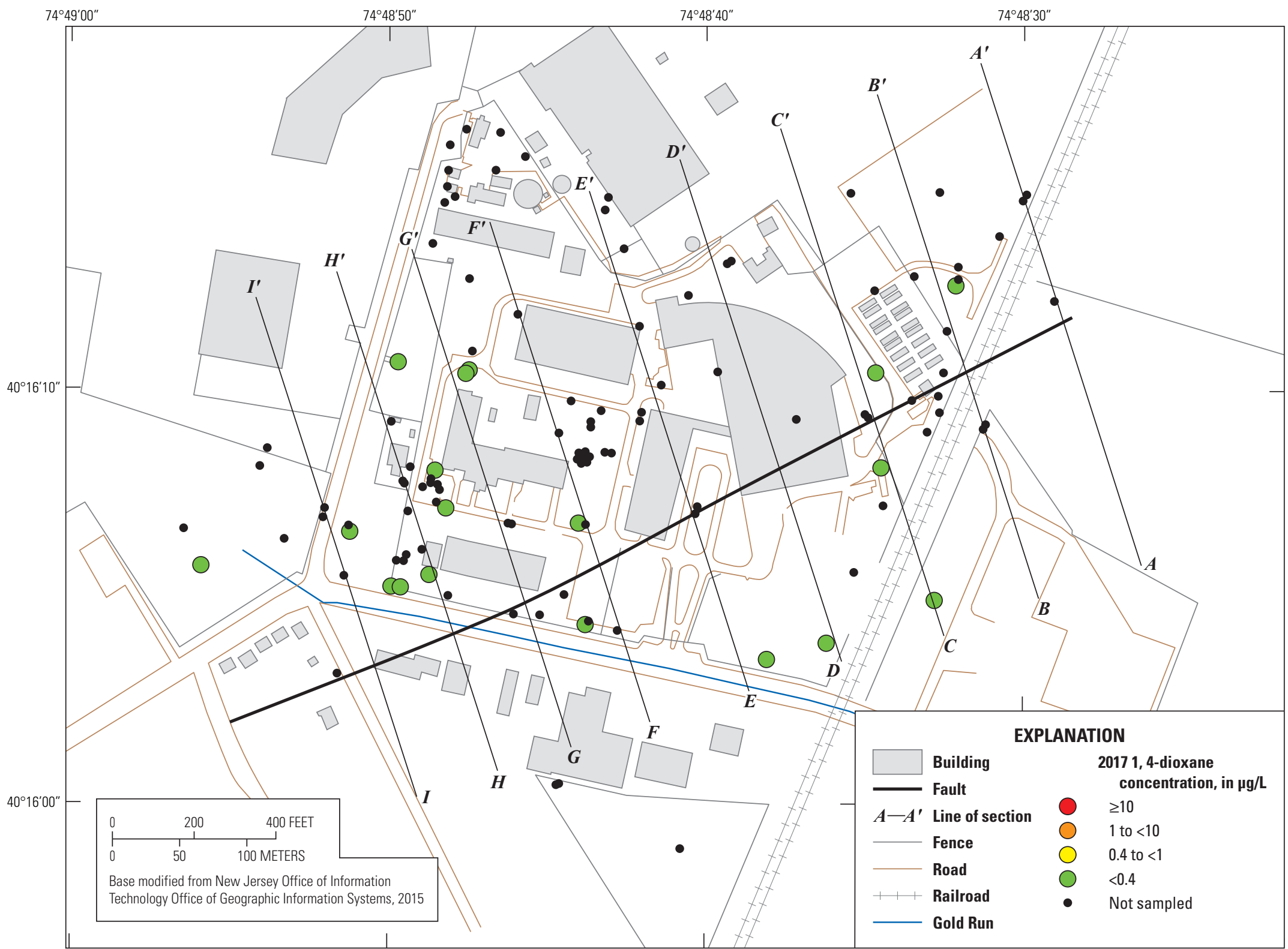

Figure 67. Areal extent of 1,4-dioxane concentrations in wells at the former Naval Air Warfare Center, West Trenton, New Jersey, 2017. (mg/L, micrograms per liter) 
Contamination by PFASs was first detected in extraction well 48BR in the eastern area at NAWC in 2015. Perfluorooctane sulfonate (PFOS), perfluorooctanoic acid (PFOA), and perfluorononanoic acid (PFNA) were the only PFAS compounds quantified in the sample. In 2016, all pump-and-treat extraction wells plus five other wells were sampled for PFAS. Well 48BR was again contaminated with PFAS compounds, while the other extraction wells at the NAWC site had concentrations at less than detection levels or were estimated to be near detection levels of these three PFAS compounds. Three other monitoring wells contained above detection PFOS, PFOA, and PFNA concentrations in the Site 3 area in 2016. In 2017, PFOS, PFOA, and PFNA concentrations greater than their New Jersey Department of Environmental Protection Maximum Contaminant Levels (MCLs) were detected in 25, 24, and 21, respectively, of the 26 wells sampled on the eastern side of NAWC. PFOS was the perfluorinated compound found in the highest concentration in all PFAS-contaminated wells. PFOS concentrations were highest in shallow wells $11 \mathrm{~S}(26,000 \mathrm{ng} / \mathrm{L})$ and $27 \mathrm{~S}(27,000$ $\mathrm{ng} / \mathrm{L}$ ) along the fence north of the tank farm, the area where the AFFF was purported to have been discharged in the past. PFOA and PFNA concentrations were detected only in wells where PFOS concentrations were detected, but at concentrations that were $1-3$ orders of magnitude less than the PFOS concentrations. PFOS and PFOA were detected in four wells east of the railroad tracks, indicating that groundwater contaminated with these compounds had flowed off the NAWC site. PFOS and PFNA concentrations $\geq 13 \mathrm{ng} / \mathrm{L}$ and PFOA concentrations $\geq 14 \mathrm{ng} / \mathrm{L}$ (their established MCLs) were present in 11,11 , and 8 wells respectively, south of the fault. It is likely that PFAS contamination traveled with shallow groundwater through the overburden or with surface-water runoff through leaking storm sewers to spread from the northern side of the fault to the southern side of the fault. Detectable concentrations of PFASs extended vertically from shallow groundwater to as deep as $200 \mathrm{ft}$ in well 54BR. Deeper wells were likely contaminated by downward seepage of PFAS-contaminated groundwater along the higher transmissivity bedding partings in the fractured rock aquifer.

In a limited test of five wells, samples collected with RCDM passive samplers contained PFAS concentrations equal to those in samples from low-flow purging. Eighteen wells sampled for 1,4-dioxane at NAWC were found to have concentrations of less than $0.4 \mu \mathrm{g} / \mathrm{L}$ in 2017. Therefore, 1,4-dioxane contamination does not appear to be a problem at the site.

\section{References Cited}

Barrio-Lage, G., Parsons, F.Z., Nassar, R.S., and Lorenzo, P.A., 1986, Sequential dehalogenation of chlorinated ethenes: Environmental Science and Technology, v. 20, no. 1, p. 96-99, accessed August 26, 2019, at https://doi.org/10.1021/es00143a013.

Bradley, P.M., Lacombe, P.J., Imbrigiotta, T.E., Chapelle, F.H., and Goode, D.J., 2009, Flowpath independent monitoring of reductive dichlorination potential in a fractured rock aquifer: Ground Water Monitoring and Remediation, v. 29, no. 4, p. 46-55, accessed August 26, 2019, at https://doi.org/ 10.1111/j.1745-6592.2009.01255.x.

Cohen, R.M., Mercer, J.W., and Matthews, J., 1993, DNAPL site evaluation: Boca Raton, Florida, C.K. Smoley, 384 p.

EA Engineering, Science, and Technology, Inc., 2000, Final decision document for ground water at the Naval Air Warfare Center, Aircraft Division, Trenton, N.J: Berkeley Heights, N.J., EA Engineering, Science, and Technology, Inc., $50 \mathrm{p}$.

ECOR Solutions, Inc., 2005, Long-term monitoring plan for the former Naval Air Warfare Center, Trenton, New Jersey: ECOR Solutions, March 14, 2005, 201 p.

Fiore, A.R., and Imbrigiotta, T.E., 2021, Concentration of chlorinated volatile organic compounds and per- and polyfluoroalkyl substances in groundwater and surface water, former Naval Air Warfare Center, West Trenton, New Jersey, U.S. Geological Survey data release, https://doi.org/ 10.5066/P9RCAQ5N.

Geosyntec Consultants, 2010, Bioaugmentation pilot study, former NAWC Trenton site, West Trenton, NJ: Acton, Mass., Geosyntec Consultants, Final Draft Report, Project number JR0021, May 2010, 41 p.

Goode, D.J., Imbrigiotta, T.E., and Lacombe, P.J., 2014, High-resolution delineation of chlorinated volatile organic compounds in a dipping, fractured mudstone-Depth- and strata-dependent spatial variability from rock-core sampling: Journal of Contaminant Hydrology, v. 171, p. 1-11, accessed August 26, 2019, at https://doi.org/10.1016/j .jconhyd.2014.10.005.

Imbrigiotta, T.E., and Harte, P.T., 2020, Passive sampling of groundwater wells for determination of water chemistry: U.S. Geological Survey Techniques and Methods, chap. 8, section D, book 1, 80 p., accessed May 13, 2021, at https://doi.org/10.3133/tm1d8. 
Imbrigiotta, T.E., Trotsky, J.S., and Place, M.C., 2008, Protocol for use of regenerated cellulose dialysis membrane diffusion samplers (ER-0313): ESTCP Protocol Report for Project ER-0313, 135 p., ESTCP, Alexandria, VA, accessed June 5, 2019, at https://www.serdp-estcp.org/content/ download/8521/104489/file/ER-200313-Protocol.pdf.

International Technology Corporation, 1994, Remedial investigation report, Installation Restoration Program, Naval Air Warfare Center, Trenton, New Jersey 6 vols.: Edison, N.J., International Technology Corporation.

Interstate Technology \& Research Council (ITRC), 2018a, Naming conventions and physical and chemical properties of per- and polyfluoroalkyl substances (PFAS): ITRC PFAS Fact Sheet 1, 15 p., accessed March 31, 2019, at https://pfas-dev.itrcweb.org/wp-content/uploads/2020/10/ naming_conventions_508_2020Aug_Final.pdf.

Interstate Technology \& Research Council (ITRC), 2018b, Environmental fate and transport of per- and polyfluoroalkyl substances (PFAS): ITRC PFAS Fact Sheet 4, 18 p., accessed March 31, 2019, at https://pfas-dev.itrcweb.org/ wp-content/uploads/2020/10/f_and_t_508_2020Aug.pdf.

Interstate Technology \& Research Council (ITRC), 2018c, Aqueous film-forming foam (AFFF): ITRC PFAS Fact Sheet 7, 12 p., accessed March 31, 2019, at https://pfasdev.itrcweb.org/wp-content/uploads/2020/10/afff_508_ 093020.pdf.

Lacombe, P.J., 2000, Hydrogeologic framework, water levels, and trichloroethylene contamination, Naval Air Warfare Center, West Trenton, New Jersey, 1993-97: U.S. Geological Survey Water-Resources Investigations Report 98-4167, 139 p. [Also available at https://doi.org/10.3133/ wri984167.]

Lacombe, P.J., 2002, Ground-water levels and potentiometric surfaces, Naval Air Warfare Center, West Trenton, New Jersey, 2000: U.S. Geological Survey Water-Resources Investigations Report 2001-4197, 38 p. [Also available at https://doi.org/10.3133/wri014197.]

Lacombe, P.J., 2011, Mass of chlorinated volatile organic compounds removed by pump-and-treat, Naval Air Warfare Center, West Trenton, New Jersey, 1996-2010: U.S. Geological Survey Scientific Investigation Report 2011-5003, 48 p. [Also available at https://doi.org/10.3133/ sir20115003.]

Lacombe, P.J., and Burton, W.C., 2010, Hydrogeologic framework of fractured sedimentary rock, Newark Basin, New Jersey: Ground Water Monitoring and Remediation, v. 30, no. 2, p. 35-45, accessed April 22, 2018, at https://doi.org/ 10.1111/j.1745-6592.2010.01275.x.
Lorah, M.M., and Olsen, L.D., 1999, Natural attenuation of chlorinated volatile organic compounds in a freshwater tidal wetland-Field evidence of anaerobic biodegradation: Water Resources Research, v. 35, no. 12, p. 3811-3827, accessed August 26, 2019, at https://doi.org/10.1029/ 1999WR900116.

Mackay, D.M., Roberts, P.V., and Cherry, J.A., 1985, Transport of organic contaminants in groundwater: Environmental Science \& Technology, v. 19, no. 5, p. 384-392, accessed August 26, 2019, at https://doi.org/ 10.1021/es00135a001.

New Jersey Department of Environmental Protection (NJDEP), 2018a, New Jersey Department of Environmental Protection, Drinking Water Quality Institute Maximum Contaminant Level Recommendations-Perfluorooctanoic acid: NJDEP Division of Water Supply and Geoscience, March 15, 2017, accessed March 31, 2019, at https://www.st ate.nj.us/dep/watersupply/pdf/pfoa-recommend.pdf.

New Jersey Department of Environmental Protection (NJDEP), 2018b, New Jersey Department of Environmental Protection, Drinking Water Quality Institute Maximum Contaminant Level Recommendations-Perfluorooctane sulfonate: NJDEP Division of Water Supply and Geoscience, June 8, 2018, accessed March 31, 2019, at https:/www.state.nj.us/dep/watersupply/pdf/pfosrecommendation-summary.pdf.

New Jersey Department of Environmental Protection (NJDEP), 2018c, New Jersey Department of Environmental Protection Drinking Water Quality Standards: NJDEP Division of Science and Research, September 4, 2018, accessed March 25, 2019, at https://www.nj.gov/dep/ standards/drinking\%20water.pdf.

NIOSH, 2007, Pocket guide to chemical hazards: Department of Health and Human Services Publication No. 2005-149, 3rd Printing, September 2007, 424 p., accessed August 26, 2019, at https://www.cdc.gov/niosh/docs/2005-149/ default.html.

Révész, K.M., Lollar, B.S., Kirshtein, J.D., Tiedeman, C.R., Imbrigiotta, T.E., Goode, D.J., Shapiro, A.M., Voytek, M.A., Lacombe, P.J., and Busenberg, E., 2014, Integration of stable carbon isotope, microbial community, dissolved hydrogen gas, and ${ }^{2} \mathrm{H}_{\mathrm{H} 2 \mathrm{O}}$ tracer data to assess bioaugmentation for chlorinated ethene degradation in fractured rocks: Journal of Contaminant Hydrology, v. 156, p. 62-77, accessed August 26, 2019, at https://doi.org/10.1016/j .jconhyd.2013.10.004. 
Shapiro, A.M., Tiedeman, C.R., Imbrigiotta, T.E., Goode, D.J., Hsieh, P.A., Lacombe, P.J., DeFlaun, M.F., Drew, S.R., and Curtis, G.P., 2018, Bioremediation in fractured rock-2. Mobilization of chloroethene compounds from the rock matrix: Ground Water, v. 56, no. 2, p. 317-336, accessed August 26, 2019, at https://doi.org/10.1111/gwat.12586.

Tetra Tech, 2018a, Draft Summary Report, 2017 Annual (Spring) sampling event, former Naval Air Warfare Center, Trenton, New Jersey: Norfolk, Virginia, Tetra Tech, [n. p.].

Tetra Tech, 2018b, Final evaluation of potential sources of perand polyfluoroalkyl substances, former Naval Air Warfare Center Trenton, Trenton, New Jersey: Norfolk, Virginia, Tetra Tech, [n. p.].

Tetra Tech, 2010, Sampling and analysis plan for VOC sampling and long-term monitoring at the former Naval Air Warfare Center Trenton, Trenton, New Jersey: February 2010, 96 p.

Tetra Tech, 2017, Sampling and analysis plan for per- and polyfluoroalkyl substances sampling and long-term monitoring and surface water sampling, former Naval Air Warfare Center Trenton, Trenton, New Jersey: June 2017, 126 p.

Tiedeman, C.R., Shapiro, A.M., Hsieh, P.A., Imbrigiotta, T.E., Goode, D.J., Lacombe, P.J., DeFlaun, M.F., Drew, S.R., Johnson, C.D., Williams, J.H., and Curtis, G.P., 2018, Bioremediation in Fractured Rock-1. Modeling to inform design, monitoring, and expectations: Ground Water, v. 56, no. 2, p. 300-316, accessed August 26, 2019, at https://doi.org/10.1111/gwat.12585.

U.S. Environmental Protection Agency (EPA), 2016, U.S. Environmental Protection Agency PFOA \& PFOS Drinking Water Health Advisories Fact Sheet, May 2016, accessed March 31, 2019, at https://www.epa.gov/ground-waterand-drinking-water/drinking-water-health-advisoriespfoa-and-pfos.

U.S. Environmental Protection Agency (EPA), 2018a, U.S. Environmental Protection Agency Method 8260D, Volatile organic compounds by gas chromatography/mass spectrometry: Hazardous Waste Test Methods/SW846, update VI, revision 4, June 2018, 53 p., accessed March 31, 2019, at https:/www.epa.gov/sites/production/files/ 2018-06/documents/method_8260d_update_vi_final_0611-2018.pdf.

U.S. Environmental Protection Agency (EPA), 2018b, U.S. Environmental Protection Agency Method 537A, Determination of selected per- and polyfluoroalkyl substances in drinking water by solid phase extraction and liquid chromatography/tandem mass spectrometry (LS/ MS/MS): EPA/600/R-18/352, version 1.0, November 2018, accessed March 31, 2019, at cfpub.epa.gov/si/si_public file_download.cfm?p_download_id=537290\&Lab=NERL.
U.S. Environmental Protection Agency (EPA), 2018c, U.S. Environmental Protection Agency Method 8270ESemivolatile organic compounds by gas chromatography/ mass spectrometry (GC/MS): Hazardous Waste Test Methods/SW846, update VI, revision 6, June 2018, 64 p., accessed March 31, 2019, at https:/www.epa.gov/sites/ production/files/2019-01/documents/8270e_revised_6_ june_2018.pdf.

Vogel, T.M., Criddle, C.S., and McCarty, P.L., 1987, ES Critical Reviews-Transformation of halogenated aliphatic compounds: Environmental Science \& Technology, v. 21, no. 8, p. 722-736, accessed August 26, 2019, at https://doi.org/10.1021/es00162a001.

Watermark Environmental, 2014, Sampling and analysis plan, long-term monitoring, former Naval Air Warfare Center Trenton, Trenton, New Jersey: April 2014, [n. p.].

Watermark Environmental, 2015a, Sampling and analysis plan, long-term monitoring, former Naval Air Warfare Center Trenton, Trenton, New Jersey: May 2015, [n. p.].

Watermark Environmental, 2015b, Final summary report, 2014 Annual (Spring) sampling event, former Naval Air Warfare Center, Trenton, New Jersey: Lowell, Massachusetts, Watermark Environmental Inc., [n. p.].

Watermark Environmental, 2016a, Sampling and analysis plan, long-term monitoring, former Naval Air Warfare Center Trenton, Trenton, New Jersey: May 2016, 102 p.

Watermark Environmental, 2016b, Final summary report, 2015 Annual (Spring) sampling event, former Naval Air Warfare Center, Trenton, New Jersey: Lowell, Massachusetts, Watermark Environmental Inc., [n. p.].

Watermark Environmental, 2017, Final summary report, 2016 Annual (Spring) sampling event, former Naval Air Warfare Center, Trenton, New Jersey: Lowell, Massachusetts, Watermark Environmental Inc., [n. p.].

Wiedemeier, T.H., Swanson, M.A., Moutoux, D.E., Gordon, E.K., Wilson, J.T., Wilson, B.H., Kampbell, D.H., Haas, P.E., Miller, R.N., Hansen, J.E., and Chapelle, F.H., 1998, Technical protocol for evaluating natural attenuation of chlorinated solvents in groundwater: U.S. Environmental Protection Agency 600/R-98/128, September 1998, 248 p., accessed August 26, 2019, at https://www.researc hgate.net/profile/John_Wilson37/publication/235101797 Technical_Protocol_for_Evaluating_Natural_Attenuation of_Chlorinated_Solvents_in_Groundwater_Revision_1/ links/0046351a5338dd74c8000000/Technical-Protocol-forEvaluating-Natural-Attenuation-of-Chlorinated-Solvents-inGroundwater-Revision-1.pdf. 
Wilson, J.T., Kampbell, D., Weaver, J., Wilson, B., Imbrigiotta, T., and Ehlke, T., 1995, A review of intrinsic bioremediation of trichloroethylene in ground water at Picatinny Arsenal, New Jersey, and St. Joseph, Michigan-Bioremediation of Hazardous Wastes: EPA/540/R-95/532, September 1995, p.13-16, accessed August 26, 2019, at https://play.google.com/books/reader? $\mathrm{id}=$ wiJSAAAAMAAJ\&hl=en\&pg=GBS.PR3.

Wilson, J.T., and Wilson, B.H., 1985, Biotransformation of trichloroethylene in soil: Applied and Environmental Microbiology, v. 49, no. 1, p. 242-243, accessed August 26, 2019, at https:/www.ncbi.nlm.nih.gov/pmc/articles/ PMC238380/pdf/aem00147-0258.pdf. 
Appendix 1. Descriptions of boreholes, well locations, and well construction at the former Naval Air Warfare Center, West Trenton, New Jersey 
Appendix 2. Volatile organic compounds, per- and polyfluoroalkyl substances, and 1,4-dioxane concentrations measured in samples from wells at the former Naval Air Warfare Center site, West Trenton, New Jersey, 1990-2017 

For additional information, contact:

Director, New Jersey Water Science Center U.S. Geological Survey

3450 Princeton Pike Ste 110,

Lawrenceville, New Jersey, 08648

Or visit our website at: https://www.usgs.gov/centers/nj-water

Publishing support provided by the

West Trenton Publishing Service Center. 
